\title{
RARE Velocimetry of Shear Banded Flow in \\ Cylindrical Couette Geometry
}

\author{
by \\ Stefan Kuczera
}

\begin{abstract}
A thesis
submitted to the Victoria University of Wellington in fulfilment of the requirements for the degree of Doctor of Philosophy
\end{abstract}

Victoria University of Wellington

2015 



\section{Abstract}

A flow phenomena called 'shear banding' is often observed for a certain class of complex fluids, namely wormlike micellar solutions. Wormlike micelles are elongated flexible self-assembly structures formed by the aggregation of amphiphiles, which may entangle into a dynamic network above a certain concentration threshold. The entanglement results in the sample having both solid-like (elastic) and liquid-like (viscous) properties, an ambiguity commonly found in complex fluids. Under certain shear conditions, the flow couples with the structure of the micellar network, leading to the formation of (shear) bands with differing viscosity.

The principle goal of this work is to address open questions regarding the temporal and spatial stability of shear banded flow. Shear banding is often studied in cylindrical Couette cells, where the fluid is sheared in a gap between differentially rotating concentric cylinders. For the sake of an accurate description of the flow in such a shear cell, the methodology for a 2D Nuclear Magnetic Resonance (NMR) velocimetry technique (known as PGSE-RARE), which offers high temporal and spatial resolution, is improved and refined. Two main challenges are identified and overcome. The first concerns the fact that the velocity imaging process operates on a Cartesian grid, whereas the flow in the Couette cell is of cylindrical symmetry. Numerical calculations and NMR simulations based on the Bloch equations, as well as experimental evidence, give insight on the appropriate selection of the fluid volume over which velocity information is accumulated and the preferred scheme through which the NMR image is acquired in the so-called $k$-space. The small extent of the fluid gap for the cells in use is the second challenge. In this respect, a variant of the velocimetry technique is developed, which offers ultra high resolution in the gap direction, necessary for a detailed description of the flow profile in the banded state.

The refined methodology is applied in a thorough study of a certain wormlike micellar solution (' $10 \% \mathrm{CPCl}$ '), which is known to exhibit spatiotemporal fluctuations and has been subject of numerous studies over the past 20 years. NMR results are supported by a recently developed 2D Rheo-USV (Ultrasonic Speckle Velocimetry) method, which offers an even higher temporal resolution. The two complementary methods show good agreement for averaged velocity profiles. In line with previous studies the fluid is found to follow a standard anomalous lever rule, which is characterized by a constant shear rate in the high viscosity band and a varying shear rate and proportion of the high shear rate band. In particular, the high resolution NMR variant allows a refined picture on the dynamics of the interface between the two bands. Furthermore, slip is observed for all investigated shear rates. The amount of slip, however, is found to strongly depend on the specifities of the Couette cells in use. Spatially and temporally resolved flow maps reveal various flow instabilities. Ultrasound measurements show vorticity structures in the order of the gap width. In the NMR case no such structures are observed due to the lower resolution in the axial direction. For higher shear rates the occurrence of turbulent bursts is detected for USV. No direct evidence of similar flow instabilities is found in the NMR case. Finally, broad distributions dominate the high shear rate band in temporally and spatially resolved velocity profiles, showing the fluctuative nature of the flow. 



\section{Acknowledgements}

First of all, I would like to thank my principal supervisor, Petrik Galvosas, who was a helpful guide in all aspects during the whole course of this thesis and who always encouraged me to pursue my own ideas. Sadly, my co-supervisor Paul Callaghan passed away about a year into my project. Though I did not have the joy of many discussions and encounters with him, his presence was still felt not only in our group, but also in the whole university and at conferences abroad. Bill Williams, who became my co-supervisor after Paul's passing, was delightful with his thoughtful insights on research, as well as with his superb sense of humour. In terms of financial support for my PhD position, the New Zealand Royal Society shall be acknowledged.

Special thanks goes to my co-workers on the Marsden fund Rheo-NMR project, namely Bradley Douglass and Tim Brox. Furthermore, I would like to acknowledge the nice atmosphere in our research group, which sparked numerous interesting discussions. Many group members have not only been respected colleagues, but have also become closer friends. The school of chemical and physical science (SCPS) as such provided many scientific and non-scientific activities keeping university life interesting. Furthermore, I would like to thank our overseas collaborators, namely Marc-Antoine Fardin, Christophe Perge, Sébastien Manneville (France) and Ulf Olsson (Sweden) who made me feel welcome during my research stays abroad. Also, the help and guidance of other collaborators (Suzanne Fielding, Jennifer Brown, Kirk Feindel, Claudia Schmidt) shall be acknowledged. My thesis examiners, Kate McGrath, Daniel Holland and Christian Mayer are thanked for their insightful comments and suggestions.

Outside of university life, I had the privilege to meet many interesting people during the course of this work, not only in New Zealand, but also in other parts of the world, where my research was taking me to. Particular thanks goes to all my human and feline (Fardin (2014)) flatmates over the course of the last years, which generated many joyful hours. The support by my parents and grandparents back home was also very important for me.

Finally, I would like to thank my partner for her patience and support in particular during the last stage of the thesis.

\section{Bibliography}

M. A. Fardin. On the rheology of cats. Rheolgy Bulletin, 83(2):16-17 and 30, July 2014. 



\section{Contents}

1 Introduction 1

2 Nuclear Magnetic Resonance 5

2.1 General Principles . . . . . . . . . . . . . . . . . . . . 5

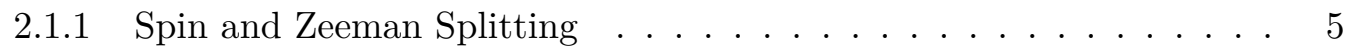

2.1.2 Bloch Vector Model . . . . . . . . . . . . . . . . . . 7

2.1 .3 Signal Detection . . . . . . . . . . . . . . . . . . 10

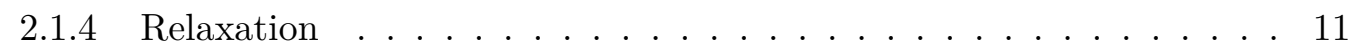

2.1.5 Spin Echo and CPMG Train . . . . . . . . . . . . . 13

2.1.6 Stimulated Echo . . . . . . . . . . . . . . . . 17

2.1.7 Coherence Pathways . . . . . . . . . . . . . . . . . . . 18

2.1.8 Magnetic Field Gradients . . . . . . . . . . . . . . . . . . . . 20

2.1.9 Selective Excitation . . . . . . . . . . . . . . . . . . 21

2.2 Magnetic Resonance Imaging and Velocimetry . . . . . . . . . . . . . . . . 22

2.2 .1 Magnetic Resonance Imaging . . . . . . . . . . . . . . . . . . . 22

2.2.2 Magnetic Resonance Velocimetry . . . . . . . . . . . . . . . . 25

2.2 .3 Fast imaging techniques . . . . . . . . . . . . . . . . 27

3 Rheology of Wormlike Micelles 37

3.1 Principles of Shear Rheology _ . . . . . . . . . . . . . . . . . . . 37

3.1 .1 Hookean Solid . . . . . . . . . . . . . . . . 37

$3.1 .2 \quad$ Newtonian Fluid . . . . . . . . . . . . . . . . . . 38

3.1.3 Maxwell model . . . . . . . . . . . . . . . . . . . . . . . . . 40

3.1.4 Tensorial Character and Dimensionless Numbers . . . . . . . . . . . 41

3.2 Rheometry . . . . . . . . . . . . . . . . . . . 43

3.2.1 Shearing Geometries . . . . . . . . . . . . . . . . 43

3.2 .2 Flow Curve . . . . . . . . . . . . . . . . . . . 47

3.2 .3 Slip . . . . . . . . . . . . . . . . . . . 47 
3.3 Surfactants and Wormlike Micelles . . . . . . . . . . . . . . . . . . 48

3.3.1 Surfactants in Solution . . . . . . . . . . . . . . . . 48

3.3 .2 Wormlike Micelles . . . . . . . . . . . . . . . . . . 50

3.4 Shear Banding in Wormlike Micelles . . . . . . . . . . . . . . . 53

4 Simulation of NMR Signal $\quad 59$

4.1 RARE sequence simulation . . . . . . . . . . . . . . 59

4.2 Soft pulse simulation . . . . . . . . . . . . . . . . 65

4.2.1 Underlying Bloch Equations and Numerical Solver . . . . . . . . . 65

4.2 .2 Simulation Procedure . . . . . . . . . . . . . . . 66

4.2 .3 Comparison to 'imslice' Experiment . . . . . . . . . . . . . 68

5 RARE Velocimetry for Cylindrical Couette Flow 73

5.1 Design of Rheo-NMR Hardware . . . . . . . . . . . . . . . . . . . 74

5.2 Theory and Implementation . . . . . . . . . . . . . . . 76

5.2.1 The PGSE-RARE pulse sequence . . . . . . . . . . . . . 76

5.2 .2 Slice Selection _ . . . . . . . . . . . . . . . . 79

5.2 .3 Fluid Motion Artefact . . . . . . . . . . . . . . . . . . 87

5.2 .4 Phase Cycling . . . . . . . . . . . . . . . . . . . 92

5.2.5 Determination of Gap Position . . . . . . . . . . . . . . . . . 92

5.2.6 Measurement Timescales and Relaxation . . . . . . . . . . . . 95

5.3 Experimental . . . . . . . . . . . . . . . . . . 96

5.3 .1 Validation . . . . . . . . . . . . . . . 96

5.3.2 Centric Scheme and Structures in the Phase Direction . . . . . . . . 99

5.3 .3 High Resolution Method . . . . . . . . . . . . . . . . . . . 101

5.4 Conclusion . . . . . . . . . . . . . . . . . . . . 108

6 Rheo-NMR and Rheo-USV of $10 \% \mathrm{CPCl} 113$

6.1 The Sample . . . . . . . . . . . . . . . . . . . . . . . . . . . . 114

6.1 .1 Previous Studies . . . . . . . . . . . . . . . . 114

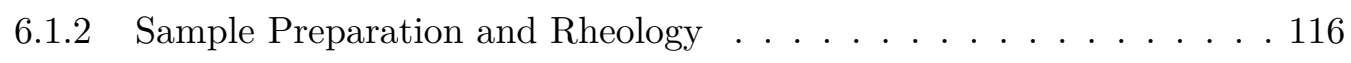

6.2 Experimental . . . . . . . . . . . . . . . . . . . . . 117

6.2 .1 Rheo-NMR . . . . . . . . . . . . . . . . . 117

6.2 .2 Rheo-USV . . . . . . . . . . . . . . . . . . . . . . 119

6.3 Results . . . . . . . . . . . . . . . . . . . . 122

6.3.1 Averaged Velocity Profiles . . . . . . . . . . . . . . . . . . 123

6.3.2 Higher Dimensions: Vorticity Undulations and Temporal Fluctuations133 
6.4 Conclusions . . . . . . . . . . . . . . . . . . . . . . . . . 143

7 Summary and Outlook $\quad 149$

7.1 Summary . . . . . . . . . . . . . . . . . . . . . 149

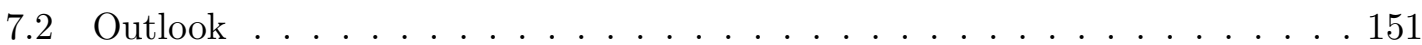

$\begin{array}{ll}\text { List of Figures } & 155\end{array}$

$\begin{array}{ll}\text { List of Tables } & 167\end{array}$ 



\section{Introduction}

Gaining insight into the complexity of soft matter is an ongoing challenge for physics. Soft matter or soft materials are often structured on multiple length scales giving rise to rich dynamics that in turn have to be understood on different timescales. Of special interest in soft matter are complex fluids, whose flow behaviour is strongly affected by their microscopic structure. Flow of complex fluids is encountered in numerous situations for everyday life, as for example while squeezing toothpaste out of the tube or spreading butter on bread. Toothpaste and butter might not be intuitively regarded as fluids, as they do also exhibit properties which would normally be associated with solids. It is this ambiguity of having both liquid-like and solid-like properties that is commonly found in complex fluids. Because the range of complex fluids is sheer endless, research is often undertaken on samples whose complexity is limited and well understood. An example are wormlike micellar solutions, which are composed of microscopic aggregates resembling wormy creatures. These aggregates, also called micelles, can form an entangled network under certain conditions making the solution elastic to a certain extent, a property usually ascribed to solids. In the case where this elastic network is subjected to a shear flow, an effect called shear banding can emerge, where the fluid is separating into two phases of differing viscosity, resulting in a peculiar flow field. A plethora of experimental techniques such as flow birefringence, dynamic light scattering, ultrasonic speckle velocimetry (USV), particle imaging velocimetry (PIV) and Rheo-NMR have been used to measure and characterize the banded flow of wormlike micellar systems (see e.g. Manneville (2008)).

Amongst these techniques Rheo-NMR stands out for its versatility and non-invasiveness (Callaghan (2007)). 'Rheo' refers to rheology, the science generally concerned with flow and deformation of matter (Barnes and Hutton (1989)). Apart from the possibility to measure flow velocities, NMR spectroscopy allows the microstructure of the studied fluid to be probed on the molecular level (Callaghan (2007)). For the present work however, the focus is mainly on the quantitative measurement of flow velocities, a technique generally known as 'velocimetry'. In particular, an effort is made to generate spatially and temporally resolved velocity maps for a certain wormlike micellar solution sheared in a cylindrical Couette cell, a device commonly used in the field of rheology (Macosko (1994)). To this 
end, fast imaging is provided by an NMR technique that also has wide-spread application in the clinical sector.

The underlying motivation for this thesis are open questions related to spatial and temporal fluctuation of shear banded flow. In particular, findings by Feindel and Callaghan (2010) call for further investigations. These authors reported on a very unusual shear banding behaviour of a micellar solution using the same NMR technique employed here. Their results were controversially discussed thereafter by Fardin et al. (2012). The present work tries to bridge between those two studies by delivering new insights and by giving arguments as to why different observations might have been made in each of the cases. In doing so, a careful analysis of experimental parameters is given, seeking for an accurate and artifact-free NMR flow measurement. This analysis is supported by numerical NMR simulations developed by the author. Furthermore, a new Rheo-USV technique (Gallot et al. (2013)) is applied to the same system for the sake of a more complete study. The latter technique also allowed for the characterization of rapid flow fluctuations that have been observed for the studied wormlike micellar system. To this end, the thesis is structured in the following way:

Chapter 2 gives a general description on the underlying principles of NMR, that are important for the thesis. Special attention is paid to the Bloch vector model, which is a common tool for explaining NMR imaging experiments. Furthermore, key aspects of the imaging sequence used in this work (RARE) are introduced and discussed, such as the relevance of coherence pathways.

Chapter 3 is concerned with the rheology of wormlike micelles. Firstly, basic principles of rheology are outlined using simple models such as the Newtonian and Maxwell fluid. Secondly, the cylindrical Couette cell, which is the shear geometry of main interest, is introduced. Thirdly and lastly, the behaviour of wormlike micelles is described along with a short survey on the effect of shear banding.

In Chapter 4 the functionality of two NMR simulation programs developed by the author is described. These include a program used to simulate the signal generated by the pulse sequence employed for the velocimetry experiments and a simulation allowing the study of the evolution of magnetization under so-called soft pulses, a radiofrequency pulse commonly applied in magnetic resonance imaging (MRI) experiments.

The methodology of the NMR velocimetry technique described in Chapter $\mathbf{5}$ is aimed at an accurate measurement of flow velocities in cylindrical Couette geometries. A careful inspection of experimental parameters is done with the help of the simulation programs developed in the previous chapter. Furthermore a high resolution method variant of the pulse sequence is introduced. 
Using the methodology developed in chapter 5 , shear banding for a specific wormlike micellar system is investigated in Chapter 6. The study is supported by an ultrasound technique, which is briefly described in this chapter. Presented results start off with averaged velocity profiles, followed by the discussion of structures and patterns observed in time and space.

\section{Bibliography}

Howard A. Barnes and John Fletcher Hutton. An Introduction to Rheology. Elsevier, 1989. ISBN 9780444871404.

Paul T. Callaghan. Rheo-NMR: a new window on the rheology of complex fluids. In eMagRes, pages 737-750. John Wiley \& Sons, Ltd, 2007. ISBN 9780470034590. URL http://onlinelibrary.wiley.com/doi/10.1002/ 9780470034590.emrstm0470.pub2/abstract.

M. A. Fardin, T. Divoux, M. A. Guedeau-Boudeville, I. Buchet-Maulien, J. Browaeys, G. H. McKinley, S. Manneville, and S. Lerouge. Shear-banding in surfactant wormlike micelles: elastic instabilities and wall slip. Soft Matter, 8(8):2535-2553, 2012. doi: 10.1039/C2SM06992J. URL http://dx.doi.org/10.1039/C2SM06992J.

Kirk Feindel and Paul Callaghan. Anomalous shear banding: multidimensional dynamics under fluctuating slip conditions. Rheologica Acta, 49(10):1003-1013, 2010. ISSN 00354511. URL http://dx.doi.org/10.1007/s00397-010-0470-9. 10.1007/s00397-010$0470-9$.

Thomas Gallot, Christophe Perge, Vincent Grenard, Marc-Antoine Fardin, Nicolas Taberlet, and Sébastien Manneville. Ultrafast ultrasonic imaging coupled to rheometry: Principle and illustration. Review of Scientific Instruments, 84(4):045107-045107-12, April 2013. ISSN 00346748. doi: doi:10.1063/1.4801462. URL http://rsi.aip.org/ resource/1/rsinak/v84/i4/p045107_s1?bypassSSO=1.

Christopher W. Macosko. Rheology: principles, measurements, and applications. VCH, 1994.

Sébastien Manneville. Recent experimental probes of shear banding. Rheologica Acta, 47 (3):301-318, April 2008. ISSN 0035-4511, 1435-1528. doi: 10.1007/s00397-007-0246-z. URL http://link.springer.com/article/10.1007/s00397-007-0246-z. 



\section{Nuclear Magnetic Resonance}

The work of two American research groups in the years 1945 and 1946 laid the foundation for a huge branch of science revolving around an effect known as nuclear magnetic resonance (NMR). Purcell et al. (1946) observed for the first time a considerable absorption of radiofrequency energy by a solid sample (paraffin) at a magnetic field strength specific to the studied nucleus. In the work by Bloch et al. (1946) it was shown that the irradiated substances (water, paraffin) not only absorb but also emit electromagnetic waves at frequencies linked to the strength of the magnetic field. Both Purcell and Bloch were awarded the physics Nobel prize in 1952 for their discoveries. NMR has since proven to be an important tool for chemical analysis, a field known as NMR spectroscopy. The studies by Lauterbur (1973) and Mansfield and Grannell (1973) showed that NMR could also be used to extract spatially resolved information on the studied object, a technique nowadays known as magnetic resonance imaging (MRI). MRI has found wide-spread application, most notably in the clinical sector.

This chapter introduces the basic principles of NMR and MRI. After a short survey on the Zeeman effect, the semi-classical Bloch vector model is explained. This model is very helpful in describing a range of NMR related effects which are of importance for the present work. With regards to MRI, the concept of $k$-space is outlined. Also, it shall be explained how velocities can be measured and spatially resolved using MRI. Finally, the fast imaging protocol mainly used in this work is introduced. A more complete description on NMR can be found, for example, in Callaghan (2011) and Levitt (2008).

\subsection{General Principles}

\subsubsection{Spin and Zeeman Splitting}

The nature of nuclear magnetism is inherently quantum mechanical with the fundamental property describing the nuclear spin being the spin quantum number $I$. This number, also called the spin, is quantized in increments of $\frac{1}{2}$ and specific to the nucleus. For example, the proton $\left({ }^{1} \mathrm{H}\right.$ nucleus) or the ${ }^{13} \mathrm{C}$ nucleus have spin $I$ of $\frac{1}{2}$ whereas $I=1$ for the deuteron 
( ${ }^{2} \mathrm{H}$ nucleus). A nucleus of spin quantum number $I$ can adopt any of the $2 I+1$ states in an interval between $-I$ and $I$. In the absence of an external field all states correspondent to the same energy level and are therefore degenerate. Degeneracy is broken, however, if the nucleus is subject to a magnetic field, as slight energy differences are present between the states in this situation. This phenomenon is known as the Zeeman effect. The energy difference between two neighbouring states is given by

$$
\Delta E=\frac{h}{2 \pi} \gamma B_{0}
$$

where $h$ is the Planck constant, $\gamma$ the gyromagnetic ratio specific to the nucleus and $B_{0}$ the magnetic field strength. In Fig. 2.1 the Zeeman diagram for the hydrogen nucleus is shown.

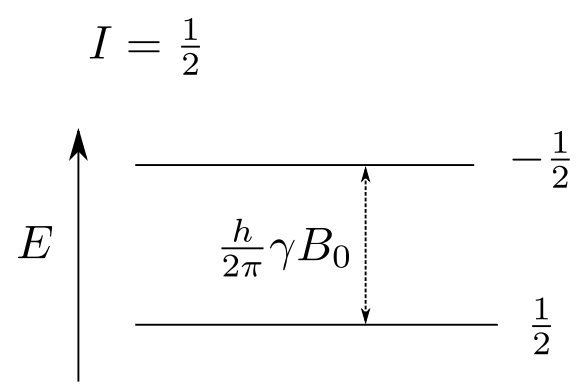

Fig. 2.1: Zeeman diagram for a nuclei with $I=\frac{1}{2}$. The lower energy state has a slightly higher probability of being occupied.

For a spin- $\frac{1}{2}$ ensemble in thermal equilibrium the populations of the spin up $\left(\frac{1}{2}\right)$ and spin down $\left(-\frac{1}{2}\right)$ state are given by the Boltzmann distribution:

$$
\frac{N_{\downarrow}}{N_{\uparrow}}=e^{-\Delta E / k_{B} T}
$$

with $k_{B}$ being the Boltzmann constant and $T$ the absolute temperature. Even for the high field system $\left(B_{0}=9.4 \mathrm{~T}\right)$ used in the present work the relative difference between populations is only about $10^{-5}$. Nonetheless, it is the small net magnetization resulting from this population difference that gives rise to the effect of nuclear magnetic resonance. Consequently no NMR signal can be received from nuclei with $I=0$. This means that common nuclei such as ${ }^{12} \mathrm{C},{ }^{14} \mathrm{~N}$ and ${ }^{16} \mathrm{O}$ cannot be used for NMR studies. However, isotopes with $I \neq 0$ exist for each of these elements, albeit with low natural abundance (CIAAW (2015)). In the present work, only ${ }^{1} \mathrm{H}$ is considered. 
Finally it shall be pointed out that the angular frequency

$$
\omega_{0}=\gamma B_{0}
$$

appearing in Eq. (2.1) is called Larmor frequency. In fact, it can be shown that the spin state is precessing around the axis of the magnetic field with exactly this frequency. This effect manifests itself also in the net magnetization vector $\mathbf{M}$, which is subject of the next section.

\subsubsection{Bloch Vector Model}

Albeit the quantum mechanical interpretation of magnetic resonance is intriguing, a semiclassical description is more convenient for the present work. In the case of independent spin- $\frac{1}{2}$ particles the temporal evolution of an ensemble of spins can be described by the precession of a bulk magnetization vector $\mathbf{M}$, analogous to a classical angular momentum. A general form of the so-called Bloch equation can be expressed as

$$
\frac{\mathrm{d} \mathbf{M}}{\mathrm{d} t}=\mathbf{M} \times \gamma \mathbf{B},
$$

where $\mathbf{B}$ is the magnetic field vector and $t$ denotes time. The description of magnetization evolution with the Bloch equations is known as Bloch vector model. In the case of a static magnetic field $\mathbf{B}_{0}=\left(0,0, B_{0}\right)$ aligned in the $z$-direction, the temporal evolution of an arbitrary magnetization vector $\mathbf{M}=\left(M_{x}, M_{y}, M_{z}\right)$ is given by

$$
\frac{\mathrm{d} M_{x}}{\mathrm{~d} t}=\gamma B_{0} M_{y}, \frac{\mathrm{d} M_{y}}{\mathrm{~d} t}=-\gamma B_{0} M_{x}, \frac{\mathrm{d} M_{z}}{\mathrm{~d} t}=0 .
$$

Using the complex notation for the transverse plane, $M_{x, y}=M_{x}+i M_{y}$, a solution for this system of coupled differential equations can be conveniently expressed as

$$
M_{x, y}=\left|M_{x, y}\right| e^{-i \gamma B_{0} t}
$$

This means that an arbitrary magnetization vector $\mathbf{M}$ is precessing around the longitudinal $(z)$ axis with the Larmor frequency $\omega_{0}=\gamma B_{0}$ under the given conditions (see Fig. 2.2a). Relaxation effects discussed in section 2.1.4 are neglected for the moment. The rotational motion of the magnetization vector can be detected and measured in an NMR experiment (section 2.1.3). However, for a spin system in thermal equilibrium the magnetization vector $\mathbf{M}$ is aligned with the magnetic field $\mathbf{B}_{0}$ and static. Therefore, the magnetization 
vector has to be moved out of its equilibrium position in order to make it detectable. This can be achieved by temporally activating another magnetic field $\mathbf{B}_{1}$ which oscillates with a frequency close or identical to the Larmor frequency of the studied nucleus. As $\mathbf{B}_{1}$ is usually only active for a short time in comparison to the total experimental time, one usually speaks of $\mathbf{B}_{1}$ pulses or alternatively rf pulses. The latter name is due to the fact that the Larmor frequency is in the radio frequency (rf) range for typical high field NMR systems.
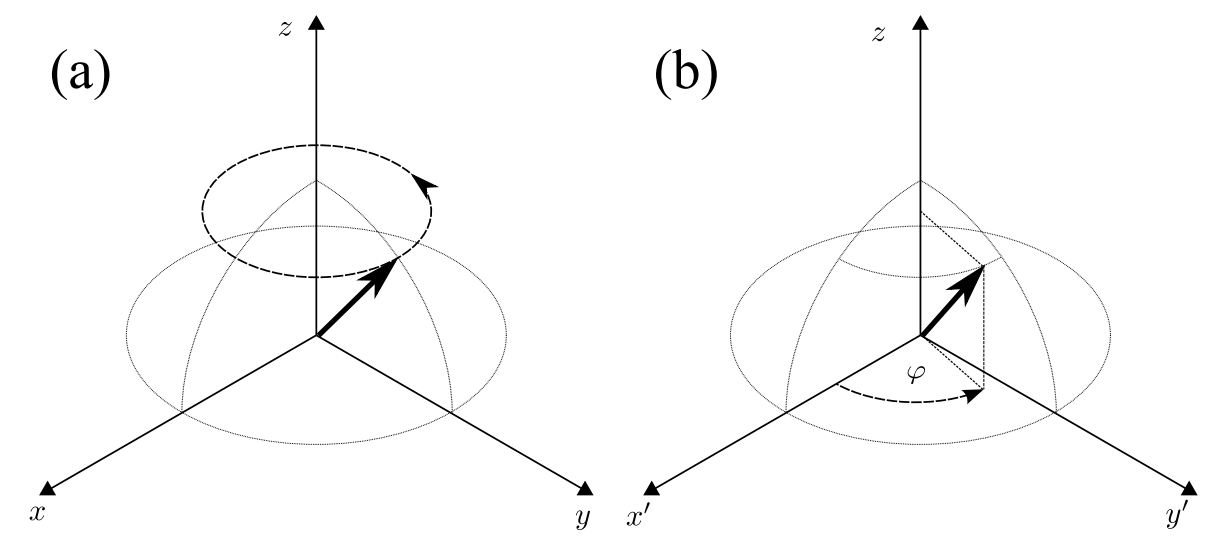

Fig. 2.2: (a) Arbitrary magnetization vector $\mathbf{M}$ precessing around the axis of the static magnetic field $\mathbf{B}_{0}$ (z-axis) in the laboratory frame. (b) Static magnetization vector $\mathbf{M}$ in the rotating frame in the on-resonant case. The angle $\varphi$ denotes the phase of the magnetization.

Before discussing the effect of an rf pulse on the magnetization vector $\mathbf{M}$, the rotating frame concept shall be introduced. In the rotating frame, the transverse $(x y)$ plane is rotating at the (angular) frequency $\omega_{\mathrm{rf}}$ of the $\mathrm{rf}$ pulse. The equation describing the precession of an arbitrary magnetization vector $\mathbf{M}$ (Eq. (2.6)) transforms to

$$
M_{x^{\prime}, y^{\prime}}=\left|M_{x^{\prime}, y^{\prime}}\right| e^{-i\left(\omega_{0}-\omega_{\mathrm{rf}}\right) t},
$$

where $M_{x^{\prime}, y^{\prime}}=M_{x^{\prime}}+i M_{y^{\prime}} . \quad M_{x^{\prime}}$ and $M_{y^{\prime}}$ are the transverse magnetization components in the rotating coordinate system. The rotating frame shall provide the perspective from which to discuss the evolution of the magnetization vector throughout the whole thesis. Hence, the prime $\left({ }^{\prime}\right)$ for $x^{\prime}$ and $y^{\prime}$ shall be dropped in the following.

In the case where the rf pulse is on-resonant, i.e. $\omega_{0}=\omega_{\mathrm{rf}}$, the transverse magnetization $M_{x, y}$ becomes static. Under these conditions the application of an rf pulse rotates the magnetization vector by an angle $\theta$ around an axis $\mathbf{e}_{\text {rot }}$ located in the transverse plane. 
Both the so-called nutation angle and the axis can be controlled by the experimenter. The nutation or tip angle is given by

$$
\theta=\frac{1}{2} \gamma B_{1} t_{\text {pulse }} .
$$

The amplitude $B_{1}$ of the rf excitation and the duration of the pulse $t_{\text {pulse }}$ can be adjusted in order to achieve the desired angle. The nutation axis $\mathbf{e}_{\text {rot }}$ on the other hand is accessible via the phase $\phi$ of the rf pulse. Using a common definition (Levitt (2008)), a phase $\phi=0$ for the rf phase corresponds to a rotation around the $x$-axis of the rotating frame. Consequently a $90^{\circ} x$-pulse rotates the magnetization by an angle of $90^{\circ}$ around the $x$-axis in the rotating frame applying the commonly accepted right-hand rule. Other typical phases and their corresponding rotation axes are summarized in table 2.1. In Fig. 2.3 the effects of $90^{\circ}{ }_{-x}, 90^{\circ}$ and $180^{\circ}{ }_{y}$ pulses are shown. In NMR jargon an rf pulse with zero phase is also called an $x$-pulse. Furthermore it is common to call the initial rf pulse that disturbs the equilibrium magnetization excitation pulse, usually being $90^{\circ}$ or less. In the case of an off-resonant rf pulse the trajectory of the magnetization vector is more complicated. For the present work however, rf pulses shall be regarded as being on-resonant if not stated otherwise.

\begin{tabular}{c|cccc}
$\phi$ & 0 & $\frac{\pi}{2}$ & $\pi$ & $\frac{3 \pi}{2}$ \\
\hline axis & $\mathrm{x}$ & $\mathrm{y}$ & $-\mathrm{x}$ & $-\mathrm{y}$
\end{tabular}

Table 2.1: Commonly defined relation between the phase $\phi$ and the rotation axis of the rf pulse in the rotating frame.
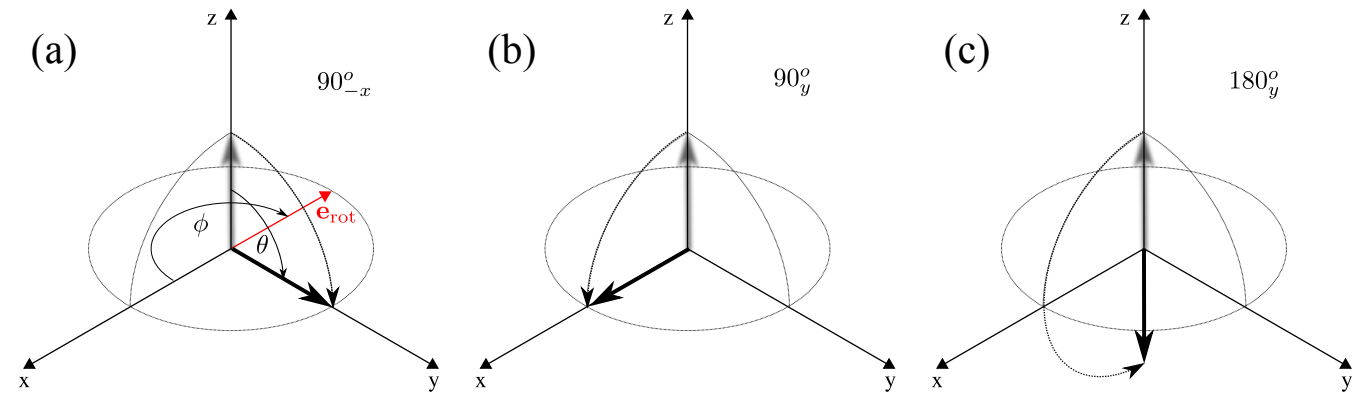

Fig. 2.3: The effect of $90^{\circ}-x$ (a), $90^{\circ}{ }_{y}$ (b) and $180^{\circ} y$ (c) pulses on an initial magnetization vector oriented in the $z$-direction. The $x$ - and $y$-axis refer to the (on-resonant) rotating frame. For (a) the rotation axis $\mathbf{e}_{\text {rot }}$, the rf phase $\phi$ and the rotation angle $\theta$ are also indicated. 
The angle $\varphi$ of the complex number $M_{x, y}$ on the Argand plane shall be referred to as the phase of the magnetization (see Fig. 2.2b). Also the static magnetic field $\mathbf{B}_{0}$ shall always be in the $z$-direction by definition. From a quantum mechanical point of view, the absorbed energy of the rf radiation changes the populations of the spin up and down states. In the case of a $180^{\circ}$ pulse a complete inversion of $N_{\downarrow}$ and $N_{\uparrow}$ is achieved.

\subsubsection{Signal Detection}

As mentioned in the previous section, it is the precession of the magnetization in the transverse plane that is measurable. The rotational motion of $\mathbf{M}$, as observed in the laboratory frame, induces a voltage in a coil usually surrounding the studied sample, an effect known as Faraday induction. The voltage itself is proportional to the magnitude $\left|M_{x, y}\right|$ and angular frequency $\omega_{0}$ of the transverse magnetization. The input voltage is phase sensitively demodulated with a frequency $\omega_{\text {ref }}$ provided by a highly stable frequency source (synthesizer). For most cases in the present work $\omega_{\text {ref }}=\omega_{\text {rf }}$, which essentially moves the acquired signal into the rotating frame. The phase sensitive demodulation of the acquired signal allows a simultaneous measurement of $M_{x}$ and $M_{y}$ and thus the signal captures the complex nature of the transverse magnetization. The recorded (complex) signal is given by

$$
S(t) \propto\left|M_{x, y}\right| e^{i \phi_{\mathrm{ref}}} e^{i \Delta \omega t}
$$

for a frequency offset $\Delta \omega=\omega_{0}-\omega_{\text {ref }}$. In most cases the (arbitrary) receiver phase $\phi_{\text {ref }}$ can either be neglected or accounted for in post-processing of the signal. To determine the resonance frequency (or frequencies) the (time-domain) signal $S(t)$ is Fourier transformed (FT), yielding a frequency spectrum (frequency domain):

$$
S(\omega)=\int_{-\infty}^{\infty} S(t) e^{i \omega t} \mathrm{~d} t
$$

Probably the most simple NMR experiment consists of single $90^{\circ}$ excitation pulse followed by subsequent recording of the signal. Due to relaxation effects discussed in section 2.1.4, the magnetization decays exponentially. The induced signal is known as the free induction decay (FID). The corresponding pulse sequence diagram and the obtained spectrum are shown in Fig. 2.4. In general, the term pulse sequence refers to a list of $\mathrm{rf}$ and gradient (see section 2.1.8) pulses that are executed in a predefined temporal order.

In practise the NMR signal is sampled and digitized at a finite rate, known as the sampling rate. The time period $t_{\mathrm{d}}$ between the acquisition of successive complex data points is called the dwell time. In accordance with the Nyquist-Shannon sampling theorem, 


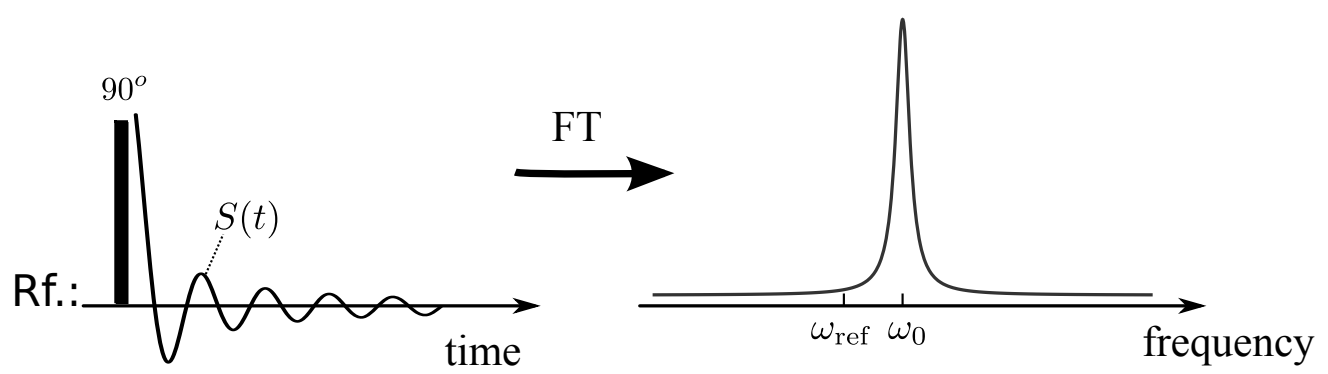

Fig. 2.4: Acquisition of an FID shown as pulse program diagram. Fourier transform of the acquired signal leads to frequency spectrum.

the sampling frequency, defined as $1 / t_{\mathrm{d}}$, must be twice as large as the maximum frequency expected in the continuous signal $S(t)$. If this condition is violated, fold-over occurs, which in particular should be avoided in magnetic resonance imaging discussed in section 2.2.1. Furthermore it is common to repeat acquisition to increase the signal-to-noise ratio (SNR) and/or to remove artefacts in the signal. To achieve the latter, the phases of the rf pulses and the receiver are varied, a procedure known as phase cycling.

\subsubsection{Relaxation}

The process by which the magnetization after an excitation is driven back into its equilibrium state is known as relaxation. One relaxation mechanism is governed by an interaction between the spin of the nucleus and its surrounding and therefore is called spin-lattice relaxation. Another important relaxation mechanism concerns the loss of coherence of the transverse magnetization known as spin-spin relaxation.

\subsubsection{Spin-Lattice Relaxation}

As mentioned earlier, rf radiation disturbs the equilibrium state of the spin ensemble by changing the populations of the spin up and down states. This population change is related to an absorption of energy from the rf pulse by the spin system. By interactions of each individual spin with its surrounding (or lattice) this energy is eventually dissipated and the ensemble returns to its equilibrium state with $\mathbf{M}_{0}=M_{0} \mathbf{e}_{z}$ as the magnetization vector. This process can be described by the following equation:

$$
\frac{\mathrm{d} M_{z}}{\mathrm{~d} t}=\frac{-\left(M_{z}-M_{0}\right)}{T_{1}} .
$$


$T_{1}$ is the time constant defining the rate of relaxation for $M_{z}$. Hence this relaxation process is also referred to as $T_{1}$ relaxation. The solution to Eq. (2.11) is given by:

$$
M_{z}(t)=\left(M_{z}(0)-M_{0}\right) e^{-t / T_{1}}+M_{0} .
$$

Please note that Eq. (2.12) fulfils the imposed boundary conditions $M_{z}(t \rightarrow \infty)=M_{0}$. Following a $90^{\circ}$ excitation pulse at $t=0$ on a system in equilibrium, $M_{z}$ is about $2 / 3$ of the equilibrium value $M_{0}$ after a relaxation period of $T_{1}$. At $t=5 T_{1}$ the relative difference between $M_{z}$ and $M_{0}$ is less than $1 \%$ and the system is usually regarded as being in equilibrium (again).

\subsubsection{Spin-Spin Relaxation}

The transverse magnetization of the whole spin ensemble precesses constantly at the fixed Larmor frequency $\omega_{0}$. Locally however, an individual spin-bearing nucleus is exposed to a slightly fluctuating magnetic field caused by the motion of surrounding particles (or spins). Hence, the precession frequency for each nuclei is also fluctuating locally causing a distribution of relative phases within the spin ensemble. As time progresses this phase distribution becomes wider leading to a loss in coherence for the spin system. In terms of the vector model, spin-spin relaxation is described by the following equation:

$$
\frac{\mathrm{d} M_{x, y}}{\mathrm{~d} t}=-\frac{M_{x, y}}{T_{2}},
$$

where $T_{2}$ is the time constant governing the decay of coherent magnetization. As pointed out by Levitt (2008), there is not necessarily an exchange of energy between the nucleus and its surrounding related to spin-spin ( or $T_{2}$ ) relaxation as is the case for spin-lattice relaxation. Furthermore, $T_{2}$ relaxation is irreversible, as the coherence decay increases the entropy (Levitt (2008)).

A similar, but reversible, coherence decay arises from the fact that, in practise, it is impossible to render a magnetic field completely homogeneous. By the same mechanism as for the $T_{2}$ relaxation, coherence is lost due to the presence of local field variations. However, as these variations can usually be regarded constant over the duration of an experiment, this decay can be reversed by applying a spin echo (see section 2.1.5). The time constant $T_{2}^{*}$ is usually used to describe the combined effect of spin-spin relaxation 
and $\mathbf{B}_{0}$ field inhomogeneity. For a perfectly homogeneous magnetic field $T_{2}^{*}=T_{2}$. Taking this relaxation mechanism into account the NMR signal of Eq. (2.9) becomes

$$
S(t) \propto\left|M_{x, y}\right| e^{i \phi_{\mathrm{ref}}} e^{i \Delta \omega t} e^{-t / T_{2}^{*}}
$$

\subsubsection{Practical Considerations}

From an experimental perspective it is usually desirable to have a short $T_{1}$ and a long $T_{2} / T_{2}^{*}$. The former as it allows for quick repetition of the NMR experiment due to a faster relaxation of the magnetization towards its equilibrium state. The latter as the strength of the recorded signal is proportional to the transverse magnetization. For sample solutions containing water, which are mainly considered in this work, it is possible to reduce the values of $T_{1}$ and $T_{2}$ by adding specific paramagnetic ions that speed up the relaxation process. These ions are known as relaxation agents. However, it is usually not possible to alter $T_{1}$ and $T_{2}$ separately as $T_{2}<T_{1}$ in all practical cases (Levitt (2008)). In terms of experimental design it is necessary to find a compromise between acceptable rates for both $T_{1}$ and $T_{2}$ relaxation. Typical values for $T_{1}$ encountered in this work range from $0.5 \mathrm{~s}$ to $1.5 \mathrm{~s}$, whereas typical values for $T_{2}$ are around $300 \mathrm{~ms}$.

Relaxation originating from an imperfect magnetic field $\mathbf{B}_{0}$ can be reduced by a process called shimming. A tunable secondary magnetic field (shim field) is imposed on the (strong) magnetic field with the aim of creating a more homogeneous field strength over the region of interest. However, it is often difficult or nearly impossible to render the magnetic field homogeneous for samples with large interfaces between phases of different susceptibility, unless the sample geometry is particularly designed for high resolution NMR. Hence, for fluids confined to the cylindrical Couette cells used herein (see section 3.2.1.1) it is hard to get $T_{2}^{*}$ close to $T_{2}$ given a reasonable time for shimming. Therefore the application of spin echoes, described in the next section, is of main importance.

\subsubsection{Spin Echo and CPMG Train}

The spin echo was originally discovered by Hahn (1950). It is generated by the application of $180^{\circ}$ pulse at a time $\tau$ after an initial $90^{\circ}$ excitation. The purpose of the $180^{\circ}$ pulse, usually called refocusing pulse, is the inversion of all phase differences inherent in the spin ensemble. The effect of a rotation by $180^{\circ}$ can be understood as a mirroring of the transverse magnetization with respect to the rotation axis. Consequently (static) field inhomogeneity that initially caused a dephasing of the magnetization between the excitation and refocusing pulse is inducing a rephasing of the magnetization in the same 
manner. After a rephasing period of $\tau$ the magnetization regains maximum coherence and the spin echo forms. The time $T=2 \tau$ is called the echo time. In Fig. 2.5 the procedure is illustrated for two different refocusing pulse phases along with the corresponding pulse sequence. 
(i)

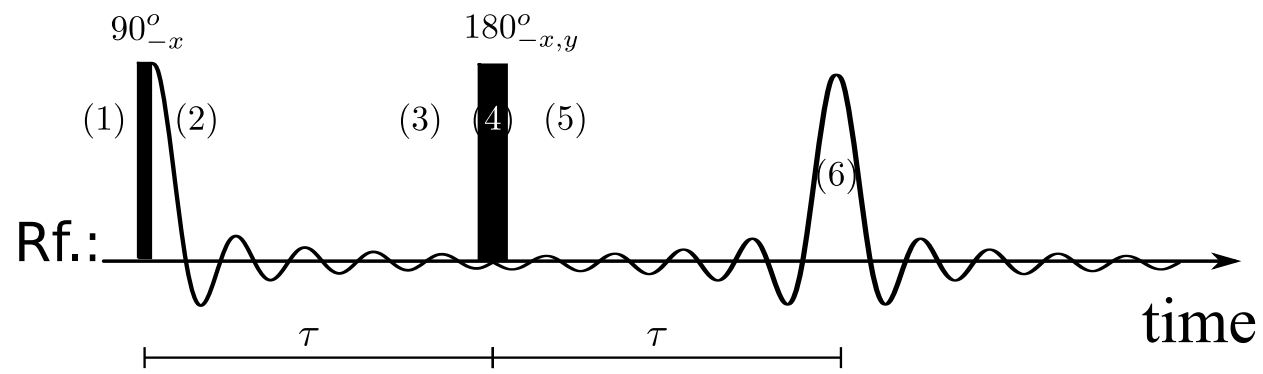

(ii)
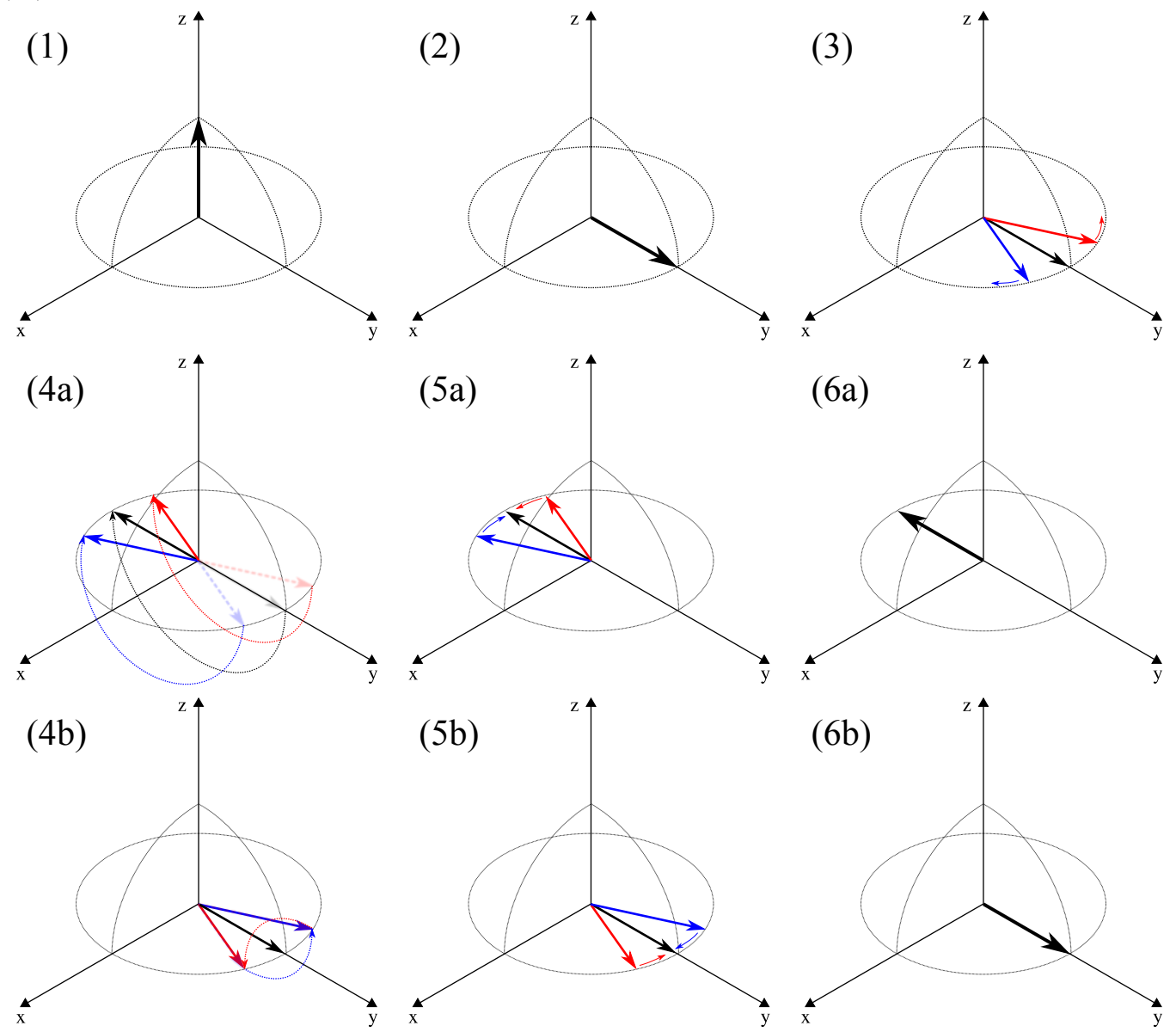

Fig. 2.5: (i) Pulse sequence used for the generation of a spin-echo. (ii) Behaviour of the magnetization under this pulse sequence for two different $180^{\circ}$ pulse phases. The magnetization initially in thermal equilibrium (1) is tipped into the transverse plane (2) by applying an excitation pulse. Due to field inhomogeneity the magnetization dephases, which can be understood as a broadening of the magnetization vector (3). The application of a $180^{\circ}$ refocusing pulse inverts the phase relation with respect to the rotational axis (4a: $-x, 4 \mathrm{~b}: y$ ). After the refocusing pulse, off-resonance effects act in the same manner and thereby rephase the magnetization (5a, 5b). At the echo time $T=2 \tau$ the magnetization is coherent again and forms a spin echo $(6 \mathrm{a}, 6 \mathrm{~b})$. Please note that the resulting magnetization vectors in case $(6 \mathrm{a})$ and $(6 \mathrm{~b})$ are antiparallel. 
Of great practical importance is the continual refocusing of magnetization via an array of $180^{\circ}$ pulses as shown in Fig. 2.6. At each integer multiple of the echo time $T$ magnetization becomes coherent again forming a spin echo. The scheme is known as CPMG sequence or CPMG train named after the scientists Carr and Purcell (1954) as well as Meiboom and Gill (1958) who studied the sequence intensively in the early days of NMR. Furthermore, the imaging sequence mainly used in this work is based on such a CPMG train.

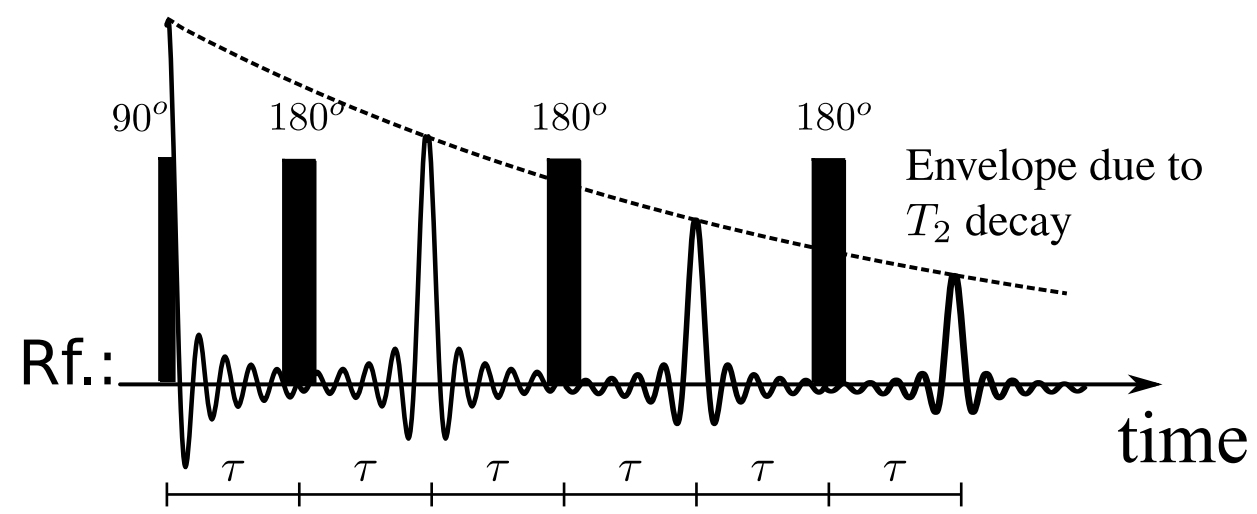

Fig. 2.6: CPMG train: An array of $180^{\circ}$ refocusing pulses is used to continually undo offresonant effects caused by field inhomogeneities. At each integer multiple of $T=$ $2 \tau$ a spin echo is formed during the train. The envelope of the echo amplitude is given by the $T_{2}$ decay if other relaxation mechanism can be neglected.

As shown in Fig. 2.6, the envelope of the echo signal can be used to measure $T_{2}$. In this respect it is advisable to have very short echo times in order to reduce relaxation-like effects due to migration of the spin-bearing nuclei in regions of different local magnetic field strength under the duration of the train. With regards to the application within an imaging sequence however (see section 2.2.3.1), the echo time is usually dictated by the duration of imaging related operations for each echo. Also, the number of echoes generated for each train is given by the parameters specific to the imaging procedure.

A particular feature of the spin echo formation as shown in Fig. 2.5 is important with regards to the CPMG sequence. Depending on the phase of the refocusing pulse the magnetization vector is either pointing in the $y$ or the $-y$ direction at point (6) where the (first) echo occurs. Following the scheme of Fig. 2.5, the magnetization vector is pointing in the $y$-direction at the second echo in both cases if the phase of the refocusing pulse is not changed. For the third echo the situation is again identical to that one of the first echo and so forth. In general, it is the transverse magnetization component perpendicular 
to the rotational axis that changes sign/parity under the application of a refocusing pulse. Hence one distinguishes between odd and even parity echoes. In the case where the $180^{\circ}$ pulses are perfect, echoes are either of odd or even parity. For imperfect refocusing pulses $\left(\theta \neq 180^{\circ}\right)$ however, the situation is more complicated. Each time a refocusing pulse is applied part of the magnetization is stored in the longitudinal direction. This induces the simultaneous formation of echoes with odd and even parity and gives rise to a peculiar temporal evolution of the net magnetization, which is discussed in section 2.1.7.

\subsubsection{Stimulated Echo}

Although stimulated echoes are not explicitly used in the present work, the underlying mechanism that leads to their formation is of relevance in understanding the evolution of the magnetization vector under a CPMG train. As shown in Fig. 2.7, the subsequent application of three $90^{\circ}$ pulses is necessary to form a stimulated echo. After the excitation by the first $90^{\circ}$ pulse the magnetization is dephasing over a period $\tau$. The second $90^{\circ}$ pulse stores a certain part of the (dephased) magnetization in the longitudinal direction. The part remaining in the transverse plane is undergoing additional dephasing during the storage period $T_{\text {store }}$ and is not of importance for the formation of the echo. The application of a third $90^{\circ}$ pulse returns the stored longitudinal magnetization back into the transverse plane where the stimulated echo is formed after a rephasing period of length $\tau$. A simple two vector model as employed in Fig. 2.5 for the spin echo is not sufficient to reason the formation of an echo as pointed out by Hennig (1991). However, as explained by the same author, using a higher number of equiangularly spaced vectors can explain how phase information is stored in the $z$-direction which eventually causes the formation of an echo. In this context the assumption is made that the magnetization is distributed equally over the transverse place at the point were the second $90^{\circ}$ pulse is applied. Although this assumption does not hold in general, it shall be assumed as being true for our purposes in the case of imaging techniques.

Stimulated echoes are preferred over spin echoes in situations where $T_{2} \ll T_{1}$, as the magnetization stored in the longitudinal direction is not undergoing $T_{2}$ relaxation. For the studies presented herein however, $T_{1}$ and $T_{2}$ are usually in the same order of magnitude. More important for the present study is the fact, that stimulated echoes are also formed during CPMG trains with imperfect refocusing pulses as shall be discussed in the next section. 


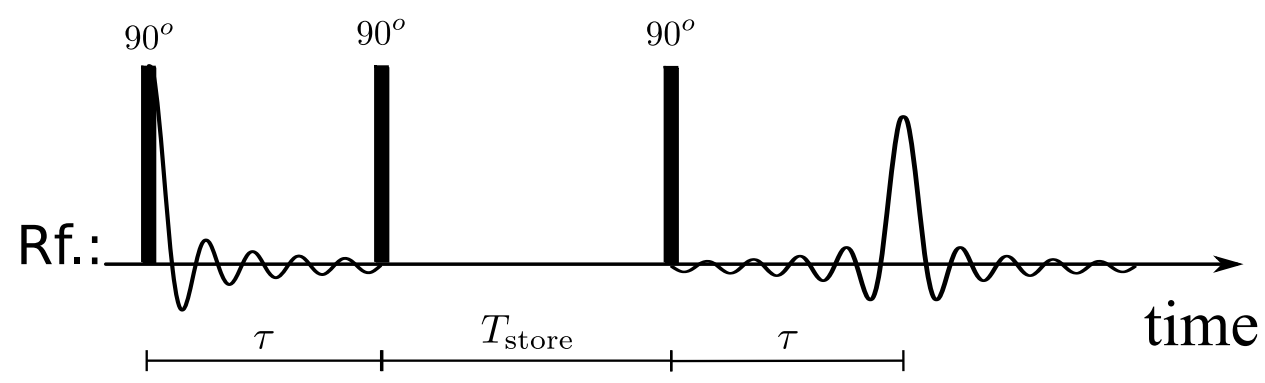

Fig. 2.7: Stimulated echo: The magnetization is first brought into the transverse plane by the initial $90^{\circ}$ pulse, where it dephases over a period $\tau$. The application of a second $90^{\circ}$ pulse transfers a certain portion of the magnetization in the longitudinal direction, where it is 'stored' over period $T_{\text {store}}$. The application of another $90^{\circ}$ pulse brings the stored magnetization back into the transverse plane where an echo is formed after another period of length $\tau$. Please note, that a simple two vector model as employed for the spin echo is not sufficient for explaining the formation of a stimulated echo.

\subsubsection{Coherence Pathways}

The imaging technique which is of main importance for the present work is based on the application of a CPMG train (section 2.1.5). Therefore the behaviour of a magnetization vector $\mathbf{M}$ under the influence of such an array of refocusing pulses shall be analysed in more detail. To this end, the slightly modified CPMG pulse sequence depicted in Fig. 2.8 is considered. In comparison to the sequence in Fig. 2.6, the $90^{\circ}$ pulse has a variable phase $\phi$ and the refocusing pulse rotates the magnetization by an angle $\alpha$ around the $y$-axis. Furthermore $T_{1}$ and $T_{2}$ relaxation shall be neglected.

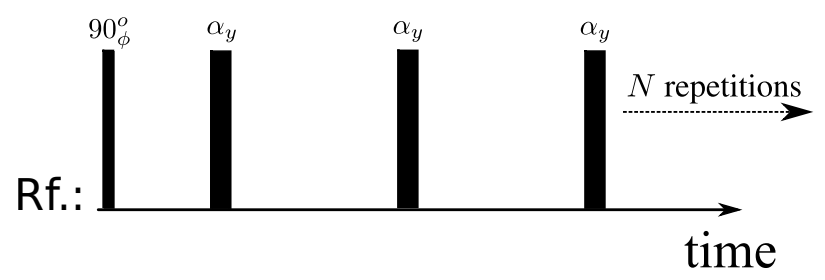

Fig. 2.8: CPMG sequence with a variable phase angle $\phi$ and nutation angles of $\alpha$ for the refocusing pulses. 
The excitation pulse rotates the equilibrium magnetization vector $\mathbf{M}_{0}=M_{0} \mathbf{e}_{z}$ into the transverse plane creating an initial magnetization

$$
\mathbf{M}(t=0)=M_{0} \cos (\varphi) \mathbf{e}_{x}+M_{0} \sin (\varphi) \mathbf{e}_{y}
$$

with $\varphi=\phi-\pi / 2$. In complex notation the transverse magnetization can be expressed as

$$
M_{x, y}(t=0)=M_{0} e^{i \varphi} .
$$

Between the excitation and the first $\alpha_{y}$ pulse the magnetization vector $\mathbf{M}(0)$ dephases due to local inhomogeneities of the magnetic field as described earlier. Assuming that $\alpha_{y}$ is not a perfect refocusing pulse, i.e. $\alpha \neq 180^{\circ}$, part of the (dephased) magnetization is stored in the longitudinal direction. Therefore the refocusing pulse is acting like a $90^{\circ}$ pulse on part of the magnetization. Generally, this deposition of magnetization in the $z$-direction occurs for all subsequent $\alpha_{y}$ pulses. Furthermore, magnetization stored in the longitudinal direction is returned into the transverse plane. As a consequence, echoes recorded for $t \geq$ $3 T$ are composed of both spin and stimulated echoes. Tracing magnetization components individually after each refocusing pulse is the idea behind the coherence pathway concept (see e.g. Kingsley (1995)). Roughly speaking, each coherence pathway corresponds to a sequence of effective rf pulses experienced by a part of the magnetization. These effective rf pulses can either be $0^{\circ}, 90^{\circ}$ or $180^{\circ}$ pulses. Consequently, the number of coherence pathways increases exponentially with the number of applied $\alpha_{y}$. Even though only part of the coherence pathways actually lead to a refocused magnetization, a considerable number of these pathways have to be taken into account after only a few echoes.

The concept of coherence pathways is useful in understanding the evolution of the magnetization vector $\mathbf{M}$ in the present case. To this end, the parallel $\left(M_{\|}\right)$and perpendicular $\left(M_{\perp}\right)$ component of $\mathbf{M}$ (with respect to $\alpha_{y}$ ) are discussed separately. The initial parallel component is given by $M_{\|}(0)=M_{0} \sin (\varphi)$, the initial component perpendicular by $M_{\perp}(0)=M_{0} \cos (\varphi)$. Numerical calculations by Hennig (1991) and theoretical considerations by Le Roux (2003) have shown that the value of $M_{\|}$at the echo position is stabilizing after only a few refocusing pulses $(\approx 7)$ for tip angles $\alpha$ as low as $30^{\circ}$. The constant exchange of longitudinal and transverse magnetization during the train leads to a quasi steady-state as echoes originating from different coherence pathways interfere constructively. Interestingly, the asymptotic value of $M_{\|}$at the echo position is given by $M_{\|}(0) \sin (\alpha / 2)$ (Hennig (1991)). The perpendicular component $M_{\perp}$ on the other hand vanishes after a certain amount of echoes depending on how strongly $\alpha$ deviates from the 
ideal $180^{\circ}$ case. Different coherence pathways create echoes of odd and even parity for this component, which interfere destructively and ultimately leaving zero net magnetization in that direction. This means that our initial magnetization vector $\mathbf{M}(0)$ is transformed into $M_{\|}(0) \sin (\alpha / 2)$ during the evolution of the pulse sequence. Apart from the experienced loss in signal strength, the phase information of the initial magnetization is lost. This result is of major importance for the present work. As shown later (section 2.2.2) information on the studied object, such as velocity, can be imprinted in the phase of the magnetization. In order to retrieve the initial phase information under these circumstances special acquisition schemes are necessary, which are also discussed later.

It shall be noted, that in conventional imaging sequences that are based on CPMG trains it is advised that the magnetization is prepared in a state so that $M_{\perp}=0$ in order to avoid signal loss during the measurement. This condition is called the 'CPMG condition' (Norris et al. (1992)). The parallel component $M_{\|}$is sometimes denoted as the 'MG-component'. Furthermore, imperfect rotation angles are encountered for soft pulses (section 2.1.9), as shall be discussed in section 5.2.2.2.

\subsubsection{Magnetic Field Gradients}

Magnetic field gradients are an essential tool for NMR imaging and velocimetry. Similar to the case of shimming, an additional magnetic field is imposed on the static $\mathbf{B}_{0}$ field, created by a so-called gradient coil surrounding the region of interest. In contrast to the shim field, the purpose of the gradient field is to deliberately render the static field inhomogeneous, albeit in a known fashion. For the present work only constant field gradients are considered, which make the magnetic field strength linearly dependent on the position along the direction of the gradient. In the presence of such a constant gradient the Larmor frequency (Eq. (2.3)) becomes a function of spatial position $\mathbf{r}$ and can be written as:

$$
\omega_{0}(\mathbf{r})=\gamma\left(B_{0}+\mathbf{G} \cdot \mathbf{r}\right)
$$

where $\mathbf{G}=\nabla B_{z}$. As these gradients can be switched rapidly, it is also common to call the periods the gradients are active 'gradient pulses'. With respect to gradients it is also useful to introduce the term isochromat. An isochromat is a localized (microscopic) sub-ensemble of spins in an inhomogeneous magnetic field that experiences the same Larmor frequency (Callaghan (2011)). In the on-resonant rotating frame, an isochromat contributes a transverse magnetization $M_{x y}(\mathbf{r}, t)$ d $\mathbf{r}$ with

$$
M_{x y}(\mathbf{r}, t)=M_{x y}(\mathbf{r}, 0) e^{i \gamma \mathbf{r} \mathbf{G} t}
$$


if relaxation or related effects are ignored between 0 and $t$. Eq. (2.18) is of central importance for the NMR imaging described in section 2.2.1. The concept of isochromats does also play a major role for the NMR simulations which are described in chapter 4 . Finally, it shall be mentioned that in order to avoid the induction of eddy current in the gradient coil, gradient pulses are switched on and off at a controlled rate, commonly by using a linear ramp.

\subsubsection{Selective Excitation}

While discussing rf pulses so far, it has been assumed that the pulse affects all spins in an identical manner. In practise this is achieved by applying a short duration (and hence wide bandwidth), high-power hard pulse. In many situations it is desirable however to only excite a certain portion of the spins in the studied object. This is achieved by using a so-called soft pulse in conjunction with the application of a magnetic field gradient. The soft pulse is of relatively long duration and low power so that only spins in a certain frequency range $\Delta \omega_{s}$ centred around $\omega_{r f}$ are coherently affected. For a constant gradient applied for example in the $z$-direction this corresponds to a nominal slice thickness

$$
\Delta z=\frac{\Delta \omega_{s}}{\gamma G_{z}} .
$$

An illustration of the principle is shown in Fig. 2.9.

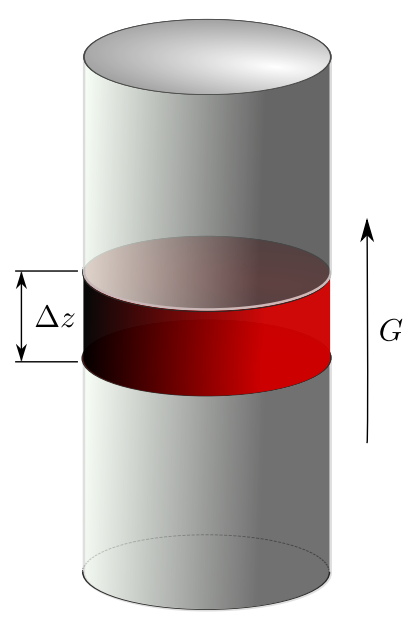

Fig. 2.9: A slice of thickness $\Delta z$ is selected out of a cylindrical object. 
Sensitivity to only a certain frequency range $\Delta \omega_{s}$ is achieved by modulating the amplitude of the soft pulse by a predefined function $f_{\text {shape }}$. The extent of the frequency window is inversely proportional to the duration of the soft pulse. The effective tip angle of the soft rf pulse can be adjusted by changing the power of the rf radiation. In the case of a small tip angle soft pulse the underlying Bloch equations can be solved analytically showing that the resulting magnetization profile over the slice is the Fourier transform of $f_{\text {shape }}$ (Callaghan (1993)). For arbitrary tip angles on the other hand, a general analytic solution is difficult to obtain. However, numerical solutions described in this work (section 4.2) and by, for example, Callaghan (1993) reveal that the results are not too dissimilar as compared to the low tip angle case. Having the Fourier relationship in mind, an ideal rectangular slice would be obtained if the modulating function is an infinite sinc function. Yet, the $B_{1}$ field of the soft pulse can only be modulated over a finite range of the sinc function causing the appearance of side lobes. For the current work, the combination of a Gaussian and a sinc function has been used as $f_{\text {shape }}$ as shall be discussed in section 4.2.3.

\subsection{Magnetic Resonance Imaging and Velocimetry}

The work in this thesis is mainly concerned with the spatially resolved measurement of fluid flow with the help of NMR, a technique called Magnetic Resonance Velocimetry (MRV). In order to obtain spatial resolution, magnetic resonance images are acquired that bear velocity information on the studied object. The art of generating images using NMR is a huge field known as Magnetic Resonance Imaging (MRI). The most prominent application of MRI lies in the clinical sector, where the non-invasive properties of magnetic resonance are advantageous for imaging the human body. Non-invasiveness is also an advantage for the measurement of flow, as interference of the measurement technique with the fluid motion is not desirable.

\subsubsection{Magnetic Resonance Imaging}

The technique of magnetic resonance imaging is based on the alteration of the local spin precession frequencies by the application of constant magnetic field gradients. According to Eq. (2.18), the signal detected from an isochromat at position $\mathbf{r}$ under the application of a constant gradient is given by

$$
\mathrm{d} S(t) \propto M_{x y}(\mathbf{r}, 0) e^{i \gamma \mathbf{r} \mathbf{G} t} \mathrm{~d} \mathbf{r}
$$


The absolute value of the proportionality constant for Eq. (2.20) is not of major importance for the description of the imaging process and shall therefore be neglected. Integrating the contributions from all isochromats yields the detected signal

$$
S(t)=\int M_{x y}(\mathbf{r}, 0) e^{i \gamma \mathbf{r} \mathbf{G} t} \mathrm{~d} \mathbf{r} .
$$

Eq. (2.21) has the form of a Fourier transform as first pointed out by Mansfield and Grannell (1973). Later this discovery led to the concept of $k$-space (Likes (1981); Ljunggren (1983); Twieg (1983)), which is extremely useful in the description of MRI experiments. In this context the reciprocal space vector, $\mathbf{k}$, shall be defined as:

$$
\mathbf{k}=\frac{\gamma \mathbf{G} t}{2 \pi}
$$

In the formalism of $k$-space, Eq. (2.21) takes the following form:

$$
S(\mathbf{k})=\int M_{x y}(\mathbf{r}, 0) e^{i 2 \pi \mathbf{k r}} \mathrm{d} \mathbf{r} .
$$

Hence by means of a (inverse) Fourier transform one obtains the initial magnetization

$$
M_{x y}(\mathbf{r}, 0)=\int S(\mathbf{k}) e^{-i 2 \pi \mathbf{k r}} \mathrm{d} \mathbf{k} .
$$

For common NMR imaging experiments, the initial magnetization is prepared in a way such that $\left|M_{x y}(\mathbf{r}, 0)\right| \propto \rho(\mathbf{r})$, where $\rho(\mathbf{r})$ is the local spin density. In this case, the magnitude of the magnetization map generated in accordance to Eq. (2.24) corresponds to a map of the local spin density, which is usually referred to as the MRI image. In general, the image defined by the initial transverse magnetization $M_{x y}(\mathbf{r}, 0)$ is a complex quantity with a phase $\varphi(\mathbf{r})$ in the Argand plane. As shown later, information on the fluid velocity can be imprinted into this phase which ultimately allows the generation of a velocity map.

As mentioned earlier, the NMR signal can only be sampled at a finite rate over a finite period. Thus it is necessary to sample the signal $S(\mathbf{k})$ in a discrete $k$-space. Most commonly this is done on a Cartesian grid, which can be represented by an n-dimensional matrix. The dimensions of the resulting image are equal to that of the grid (without zero filling). Images of maximal two dimensions (2D) are of interest for the present work. A commonly used 2D $k$-space grid of $N_{1} \times N_{2}$ points is shown in Fig. 2.10a. The components $k_{1}$ and $k_{2}$ shall correspond to two orthogonal directions in real space denoted by $x_{1}$ and $x_{2}$, respectively. The values of $k_{1,2}$ range from $-\frac{1}{2} N_{1,2} \Delta k_{1,2}$ to $\left(\frac{1}{2} N_{1,2}-1\right) \Delta k_{1,2}$ with a stepping interval of $\Delta k_{1,2}=2 k_{1,2}^{\max } / N_{1,2}$. A discrete inverse Fourier transform leads 
to conjugate real space matrix representing the image by a $N_{1} \times N_{2}$ matrix shown in Fig. 2.10b. $x_{1,2}$ ranges from $-\frac{1}{2} N_{1,2} \Delta l_{1,2}$ to $\left(\frac{1}{2} N_{1,2}-1\right) \Delta l_{1,2}$ with a stepping interval of $\Delta l_{1,2}=2 l_{1,2}^{\max } / N_{1,2}$. The resolution of the image is dictated by the Fourier relation between real space and $k$-space (or inverse space) and is given by

$$
\Delta l_{1,2}=\frac{1}{2 k_{1,2}^{\max }},
$$

i.e. by the reciprocal of the highest $k$ value in each direction. The field of view (FOV) on the other hand is the inverse of the stepping interval in $k$-space, i.e.

$$
\mathrm{FOV}_{1,2}=\frac{1}{\Delta k_{1,2}}
$$

It is important that the region from which the coherent NMR signal is expected is fully contained within the extent of the FOV. Otherwise fold-over artefacts occur where regions outside the FOV are "folded" into the image and appear on the opposite edge. Furthermore, it shall be mentioned that the main image features are contained in the centre region of $k$-space, whereas finer details are stored in the outer parts.

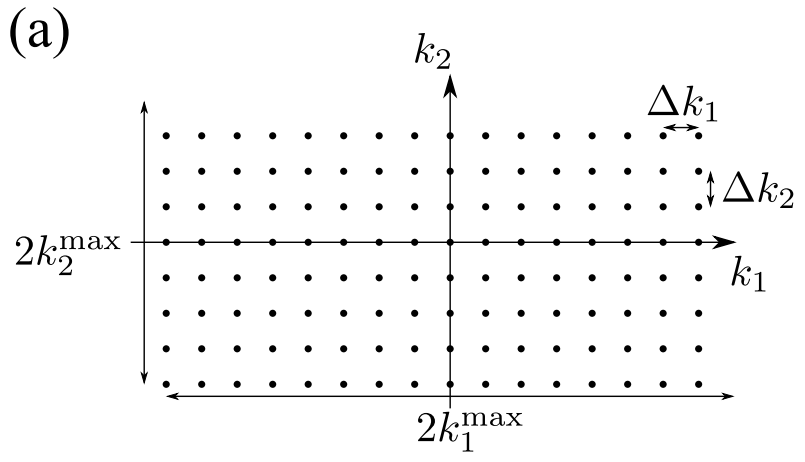

(b)

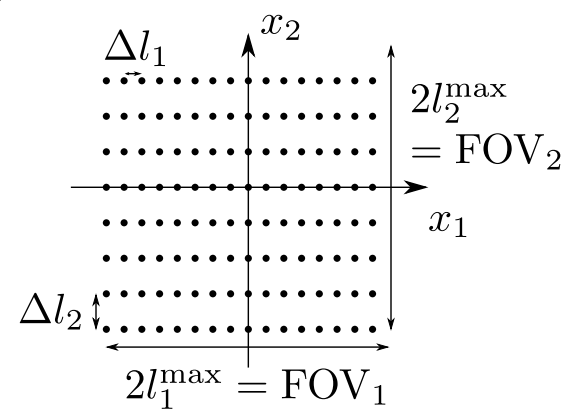

Fig. 2.10: $2 \mathrm{D}$ matrix for $k$-space (a) and real space (b). Please note, that equivalent stepping intervals $\Delta k_{1}$ and $\Delta k_{2}$ in $k$-space lead to an equivalent FOV in both spatial directions. However, the resolutions $\Delta l_{1}$ and $\Delta l_{2}$ are different due to different values for $k_{1}^{\max }$ and $k_{2}^{\max }$.

The traversal of $k$-space implies a stepping of either the gradient or the time variable. The former is known as phase encoding and involves a stepping of the gradient such that $\Delta k=\gamma \Delta G t_{p} / 2 \pi$ with $\Delta G$ being the size of the gradient steps and $t_{p}$ the gradient duration. The signal is then captured at some time $t$ after the gradient application. For the latter known as frequency encoding, the signal is captured at a rate defined by the dwell time $t_{d}$ while a gradient $G$ is applied. The corresponding step size in $k$-space is 
given by $\Delta k=\gamma G t_{d} / 2 \pi$. For fast multidimensional imaging sequences it is common to combine phase and frequency encoding. The associated directions are known as phase and read, respectively. One advantage of frequency encoding is that frequencies outside the sampling window can be suppressed by using filters. This way fold-over artefacts can be reduced in the corresponding read direction, in cases where the region of interest is not fully contained in the field of view.

So far, the effect of relaxation has not been considered. Relaxation can create image contrast (either desirable or otherwise) and cause artefacts. A common strategy is to use spin or stimulated echoes during the $k$-space acquisition process to counter effects from magnetic field inhomogeneities manifested in the $T_{2}^{*}$ time constant. The imaging sequence that is of main interest for the present work (RARE), uses a CPMG train where one line in the $k$-space is traversed per echo allowing the acquisition of the whole $k$-space grid with only one excitation. The sequence is described in more detail in section 2.2.3 along with another common fast imaging technique.

\subsubsection{Magnetic Resonance Velocimetry}

The transverse magnetization can be prepared in such a way that it bears quantitative information on the flow of the studied object. This so-called velocity encoding can be achieved with a pulse sequence known as pulsed gradient spin echo (PGSE), which is shown in Fig. 2.11. Assuming a coherent transverse magnetization in the beginning of the sequence, the first gradient pulse causes dephasing. As the refocusing pulse inverts the phase relations, the second gradient pulse subsequently rephases the magnetization. In the case where the studied object is at rest and diffusion can be neglected, the transverse magnetization is completely refocused and maintains its initial phase apart from a potential $180^{\circ}$ phase shift (see Fig. 2.5). However, if the studied object is displaced by, for example, flowing with a constant velocity $v$ in the direction of the gradient under a period $\Delta$ a net phase shift

$$
\phi=\gamma g \Delta \delta v
$$

results. The variables $\delta, \Delta$ and $g$ are defined in Fig. 2.11. Reading out the phase of the transverse magnetization after the gradient pair has been applied, allows for the determination of the flow velocity. In practical situations, field inhomogeneities introduce additional phase accrual making a velocity measurement with only one gradient value difficult. Thus, it is common practise to apply at least two values $g_{1} \neq g_{2}$ and use the difference in phase shifts for the velocity calculation. Furthermore, it is important to adjust the encoding variables in such a way that all expected phase shifts are within a $2 \pi$ 
window for each increment of gradient strength in order to ensure that each phase shift $\phi$ corresponds to a single velocity value.

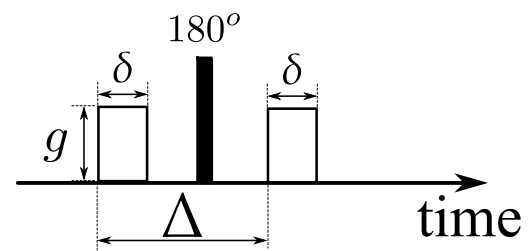

Fig. 2.11: The PGSE (pulsed gradient spin echo) sequence: Two gradients of equal length $\delta$, strength $g$ and direction with a refocusing $180^{\circ}$ pulse in between. The temporal spacing between the two gradient pulses $\Delta$ is called observation time, whereas $\delta$ is known as encoding time.

Apart from imparting a phase shift, the PGSE sequence also attenuates the magnitude of the transverse magnetization by a factor

$$
f_{a t t}=\exp \left(\gamma^{2} g^{2} \delta^{2} D(\Delta-\delta / 3)\right)
$$

known from the Stejskal-Tanner relation (Stejskal and Tanner (1965)) in the case of free diffusion with a diffusion coefficient $D$ for the material under study. Consequently, the PGSE sequence also allows for the measurement of the diffusion. Yet, diffusion processes are not of main interest for the present work. It shall be noted however that any gradient pair, even if intended for imaging purposes, introduces signal attenuation according to Eq. (2.28). As it will be discussed in section 5.3.3, diffusive attenuation can significantly decrease the signal-to-noise ratio for high resolution imaging due to the presence of strong (imaging) gradients.

For general molecular motion the propagator concept is useful (see e.g. Kärger and Heink (1983) and Callaghan (2011)). The propagator $P\left(\mathbf{r} \mid \mathbf{r}^{\prime}, \Delta\right)$ describes the probability of a spin positioned at $\mathbf{r}$ moving to $\mathbf{r}^{\prime}$ after some time $\Delta$. In analogy to $k$-space, this propagator can be measured by transversing $q$-space. Callaghan et al. (1988) define the q-vector as

$$
\mathbf{q}=\frac{\gamma \mathbf{g} \delta}{2 \pi}
$$

in the context of the PGSE sequence. Sampling a certain range of $\mathbf{q}$-vectors combined with a subsequent Fourier transform yields the propagator (or displacement spectrum). The field of flow (range of displacements that can be unambiguously detected) and the displacement resolution are defined in the same manner as for the $k$-space. Propagator 
measurements are for example useful in the case of fluctuative flow, where a whole range of fluid velocities is present. In this case the propagator can be seen as the signature of the underlying flow process. However, it should be considered that a certain amount of averaging is needed in that case in order to recover the full velocity distribution.

The strength of magnetic resonance velocimetry lies in the fact that the PGSE sequence can in theory be combined with any conventional imaging technique. After the excitation of the spin system, the motion-sensitive PGSE sequence first encodes the velocity information into the phase of the transverse magnetization and is then followed by an imaging sequence, which allows the mapping of this phase information onto an image of desired dimension. In chapter 5, the PGSE-RARE sequence, which combines the PGSE and RARE (section 2.2.3.1) sequence, is discussed in detail.

\subsubsection{Fast imaging techniques}

\subsubsection{RARE}

The rapid acquisition relaxation enhanced (RARE) sequence allows a whole $2 \mathrm{D} k$-space grid to be traversed with only one excitation (Hennig et al. (1986)). This is achieved by employing a CPMG echo train where one line of $k$-space is sampled for each echo, as shown in Fig. 2.12. After a $90^{\circ}$ excitation pulse, the magnetization is dephased by a (dephasing) read gradient, followed by a $180^{\circ}$ pulse. Between a dephasing and rephasing gradient in the phase direction the signal is acquired under the influence of a read gradient. The application of the second phase gradient closes the loop as the magnetization is in the same state as after the dephasing read gradient, disregarding relaxation and echo parity effects. Consequently, an arbitrary $k$-space line can be read out after the application of another $180^{\circ}$ pulse. As discussed in section 2.1.5, the signal magnitude is limited by the $T_{2}$ decay of the transverse magnetization. Therefore it is desirable that the time needed for the $k$-space acquisition does not exceed $T_{2}$ considerably. Otherwise lines that are sampled at later points during the sequence might be filtered out due to low signal strength.

Due to the symmetric nature of the RARE acquisition scheme, lines in $k$-space can be sampled in any thinkable order. In Fig. 2.12 two common trajectories through the inverse space are shown: linear and centric. For the linear scheme one starts at one end in $k_{\text {phase }}$ and fills lines in a linear fashion to the other end. In particular, the centre of $k$-space is sampled in the middle of the acquisition period. In the case of the centric scheme on the other hand, one starts in the centre of $k$-space and subsequently works towards the outer regions in an alternating fashion. The right choice of acquisition scheme can help to reduce artefacts in flow maps as shall be discussed in section 5.2.3.2. 


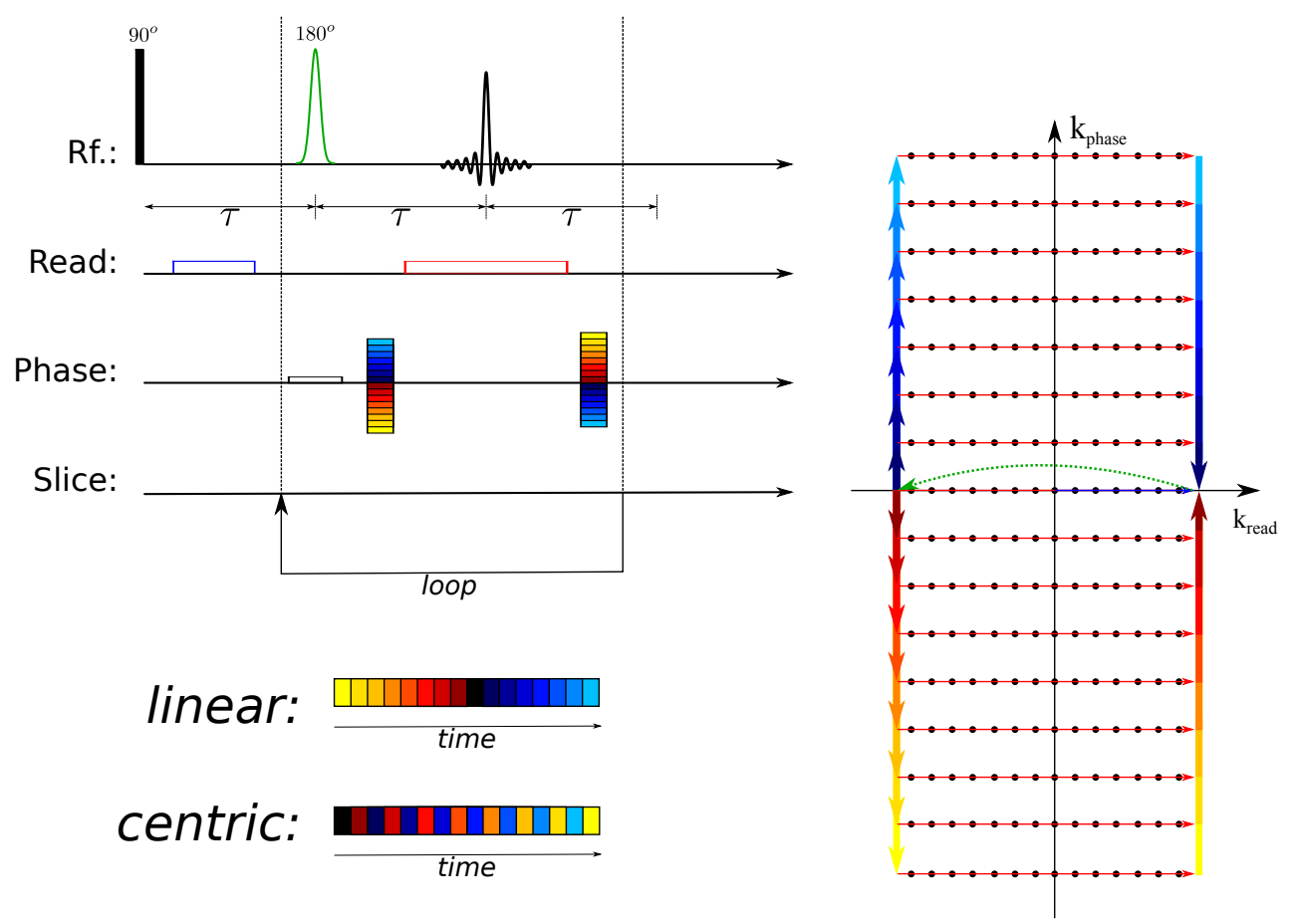

Fig. 2.12: Rapid acquisition relaxation enhanced (RARE) sequence shown along with two different $k$-space trajectories (linear and centric). Black means zero gradient strength for the acquisition schemes.

One difficulty arises with the RARE imaging sequence if a non-zero component of transverse magnetization perpendicular to the rotation axis of the $180^{\circ}$ pulse is present before the dephasing read gradient is applied. This component undergoes \pm sign modulation for odd and even echoes as already discussed in section 2.1.5. In the case of a linear $k$-space scheme this sign alteration leads to a 'ghost' image, which is displaced by half the field of view (see Callaghan (2011)). In order to avoid this artefact one can either prepare the magnetization such that the CPMG condition is fulfilled (see section 2.1.7) or use only every second echo for the acquisition of $k$-space, which in turn would double the sampling time. It shall be noted, that in the case of prior flow encoding, the CPMG condition is most likely not fulfilled, leaving only the second option. However, it has been found that the application of soft instead of hard pulses during the echo train can also reduce the appearance of ghosting artefacts (Galvosas and Callaghan (2006)).

The second difficulty concerns the fact that the $180^{\circ}$ refocusing pulses can usually not be regarded as being perfect, particularly if soft pulses are applied. As discussed in section 2.1.7 this leads to a loss of the perpendicular magnetization component during 
the course of the CPMG pulse train. Although this is not necessarily a major issue if only the signal magnitude is of interest, it has severe consequences if the magnetization has been preconditioned with a PGSE sequence. The loss of phase information essentially impedes a phase angle calculation, which is necessary for the extraction of the velocity data. This problem can be overcome by generating and adding up two images, where the phases of the refocusing pulse are offset by $90^{\circ}$ (see e.g. Galvosas and Callaghan (2006)). Essentially, this procedure, generally known as 'phase-cycling', measures the parallel and perpendicular component of the transverse magnetization individually. Adding up both components restores the original phase information.

Recently two methods have been proposed that allow maintenance of the phase information during one single CPMG pulse train. Firstly, in the 'FLIESSEN' (FLow Imaging Employing a Single-Shot ENconding) sequence by Amar et al. (2010) velocity encoding is applied individually for each echo by using a bipolar gradient pair directly after the refocusing pulse. After application of the read and phase gradients, velocity encoding is undone by an opposite bipolar gradient pair. In this way the phase imprint is renewed for every echo and therefore not subjected to destructive interference of different coherence pathways. However, FLIESSEN comes at the cost that the observation time $\Delta$ has to be included twice for each echo interval prolonging the acquisition time. Hence, it is particularly suited for fast flow where a short $\Delta$ is effective and transverse relaxation is relatively slow. Secondly, Shiko et al. (2012) presented a velocimetry method where echoes of odd and even parity are separated and thus destructive interference impeded. The sequence is based on ideas by Norris et al. (1992) and Schick (1997) and uses an unbalanced dephasing read gradient in order to achieve the separation of echoes with different parity. The acquired signal is divided into two domains from which two theoretically identical phase images can be produced and averaged to increase the signal-to-noise ratio. A disadvantage associated with the division of the signal domain is the effective reduction of the resolution by a factor of two in the read direction, as the amount of $k$-space points is halved for each separate image in comparison to the conventional RARE.

\subsubsection{Other Sequences}

Although the RARE sequence is of main interest for this work, a short overview of alternative fast imaging schemes shall be given. Among all NMR imaging techniques, 'Echo Planer Imaging' (EPI) is probably the fastest suitable for velocity measurements. The scheme of an EPI variant is shown in Fig. 2.13. The $90^{\circ}$ excitation pulse is followed by dephasing pulses in both imaging directions. After the subsequent application of a refo- 
cusing pulse, $k$-space is sampled by a series of read and phase gradients. In contrast to the RARE sequence, the whole image information is sampled in only one echo providing an enormous gain in acquisition speed. An additional advantage over RARE is that the loss of phase information due to the inference of coherence pathways is not an issue, due to the lack of a CPMG pulse train. However, considerable sensitivity to experimental imperfections are a drawback of the EPI sequence. Unwanted field inhomogeneities, mismatches in the gradients and eddy currents induced by the fast gradient switching can introduce severe artefacts. In comparison to the EPI sequence, RARE can be seen as a more robust method, as image quality does not suffer equally from experimental imperfections due to the symmetry in the acquisition process.

Rapid velocimetry using PGSE-encoded EPI was demonstrated by Kose (1994) and more recently by Sederman et al. (2004) with 'GERVAIS' (echo rapid velocity and acceleration imaging sequence), allowing a 2D flow map to be captured with a single excitation.

In an alteration of the EPI sequence, $k$-space can be sampled on a non-Cartesian grid, which in turn can result in an additional acquisition speed boost, as for example exercised by Tayler et al. (2014) in conjunction with $k$-space undersampling. Although the high temporal resolution makes EPI based velocimetry very suitable for the investigation of complex fluid flow, it has not yet been widely applied due to the amount of sophistication needed for the implementation of the sequence and the reduction of associated artefacts.
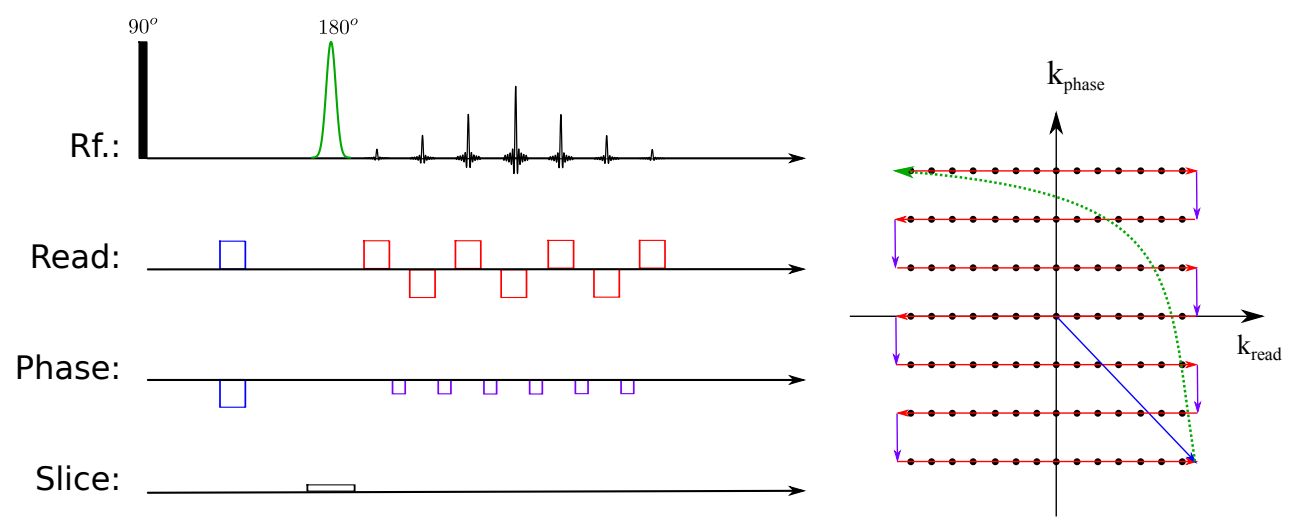

Fig. 2.13: A variant of the EPI sequence along with the employed $k$-space trajectory.

An imaging method that offers similar acquisition speed and robustness as the RARE sequence is 'Fast Low Angle SHot imaging' (FLASH) proposed by Haase et al. (1986). Here, a series of low tip angle excitations is used to cover $k$-space line by line for each 
excitation. FLASH velocimetry has been applied by Rokitta et al. (1999) to measure the xylem transport in plants.

Finally it shall be mentioned that there are also approaches that allow capture of the fluid flow without the need of phase imprinting via the PGSE sequence. These techniques commonly use a series of rapidly acquired (magnitude) images in order to reveal the dynamics of the fluid portion selected prior to acquisition. These techniques do also offer high temporal resolution, however, an extraction of quantitative velocity information is usually difficult. Examples include 'SEMI-RARE' by Sederman et al. (2003) (imaging based on RARE) and a FLASH based sequence by Moser et al. (2000).

\section{Bibliography}

Andrea Amar, Bernhard Blümich, and Federico Casanova. Rapid multiphase flow dynamics mapped by Single-Shot MRI velocimetry. ChemPhysChem, 11(12):2630-2638, 2010. ISSN 1439-7641. doi: 10.1002/cphc.201000062. URL http://onlinelibrary.wiley . com/doi/10.1002/cphc.201000062/abstract.

F. Bloch, W. W. Hansen, and M. Packard. The nuclear induction experiment. Physical Review, 70(7-8):474-485, October 1946. doi: 10.1103/PhysRev.70.474. URL http: //link.aps.org/doi/10.1103/PhysRev.70.474.

P. T. Callaghan, C. D. Eccles, and Y. Xia. NMR microscopy of dynamic displacements: k-space and q-space imaging. Journal of Physics E: Scientific Instruments, 21(8):820, August 1988. ISSN 0022-3735. doi: 10.1088/0022-3735/21/8/017. URL http://iopscience.iop.org/0022-3735/21/8/017.

Paul Callaghan. Principles of Nuclear Magnetic Resonance Microscopy. Oxford University Press, 1993. ISBN 9780198539971.

Paul T. Callaghan. Translational Dynamics and Magnetic Resonance:Principles of Pulsed Gradient Spin Echo NMR. Oxford University Press, September 2011. ISBN 9780191621048.

H. Y. Carr and E. M. Purcell. Effects of diffusion on free precession in nuclear magnetic resonance experiments. Physical Review, 94(3):630-638, May 1954. doi: 10.1103/ PhysRev.94.630. URL http://link.aps.org/doi/10.1103/PhysRev.94.630.

CIAAW. Commission on isotopic abundances and atomic weights. http://www.ciaaw.org/, 2015. URL http://www.ciaaw.org/. 
Petrik Galvosas and Paul T. Callaghan. Fast magnetic resonance imaging and velocimetry for liquids under high flow rates. Journal of Magnetic Resonance, 181(1):119-125, July 2006. ISSN 10907807. doi: 10.1016/j.jmr.2006.03.020. URL http://europepmc.org/ abstract/MED/16644252/reload=0; jsessionid=cYaR2x3QhLC6H9xa75Q0 . 12.

A Haase, J Frahm, D Matthaei, W Hanicke, and K. -D Merboldt. FLASH imaging. rapid NMR imaging using low flip-angle pulses. Journal of Magnetic Resonance (1969), 67 (2):258-266, April 1986. ISSN 0022-2364. doi: 10.1016/0022-2364(86)90433-6. URL http://www.sciencedirect.com/science/article/pii/0022236486904336.

E. L. Hahn. Spin echoes. Physical Review, 80(4):580-594, November 1950. doi: 10.1103/ PhysRev.80.580. URL http://link.aps.org/doi/10.1103/PhysRev.80.580.

J. Hennig, A. Nauerth, and H. Friedburg. RARE imaging: A fast imaging method for clinical MR. Magnetic Resonance in Medicine, 3(6):823-833, December 1986. ISSN 1522-2594. doi: 10.1002/mrm.1910030602. URL http://onlinelibrary.wiley.com/ doi/10.1002/mrm.1910030602/abstract.

Jürgen Hennig. Echoes - how to generate, recognize, use or avoid them in MR-imaging sequences. part i: Fundamental and not so fundamental properties of spin echoes. Concepts in Magnetic Resonance, 3(3):125-143, 1991. ISSN 1099-0534. doi: 10. 1002/cmr.1820030302. URL http://onlinelibrary.wiley.com/doi/10.1002/cmr . 1820030302 /abstract.

Jörg Kärger and Wilfried Heink. The propagator representation of molecular transport in microporous crystallites. Journal of Magnetic Resonance (1969), 51(1):1-7, January 1983. ISSN 0022-2364. doi: 10.1016/0022-2364(83)90094-X. URL http: //www.sciencedirect.com/science/article/pii/002223648390094X.

Peter B. Kingsley. Product operators, coherence pathways, and phase cycling part i: product operators, spin-spin coupling, and coherence pathways. Concepts in Magnetic Resonance, 7(1):29-47, January 1995. ISSN 1099-0534. doi: 10.1002/cmr.1820070103. URL http://onlinelibrary.wiley.com/doi/10.1002/cmr.1820070103/abstract.

Katsumi Kose. Spatial mapping of velocity power spectra in Taylor-Couette flow using ultrafast NMR imaging. Physical Review Letters, 72(10):1467-1470, March 1994. doi: 10.1103/PhysRevLett.72.1467. URL http://link.aps.org/doi/10.1103/ PhysRevLett.72.1467. 
P. C. Lauterbur. Image formation by induced local interactions: Examples employing nuclear magnetic resonance. Nature, 242(5394):190-191, March 1973. doi: 10. 1038/242190a0. URL http://www.nature.com/nature/journal/v242/n5394/abs/ 242190a0.html.

Patrick Le Roux. Simplified model and stabilization of SSFP sequences. Journal of Magnetic Resonance, 163(1):23-37, July 2003. ISSN 1090-7807. doi: 10.1016/ S1090-7807(03)00115-0. URL http://www.sciencedirect.com/science/article/ $\mathrm{pii/S1090780703001150.}$

Malcolm H. Levitt. Spin Dynamics: Basics of Nuclear Magnetic Resonance. John Wiley \& Sons, July 2008. ISBN 9780470517123.

Richard S. Likes. Moving gradient zeugmatography, December 1981. URL http://worldwide. espacenet. com/publicationDetails/biblio; jsessionid= 15C152373EF148F3A1D680C76C52DFAF . espacenet_levelx_prod_3?FT=D\&date= $19811222 \& D B=\& l o c a l e=e n \_E P \& C C=U S \& N R=4307343 A \& K C=A \& N D=1 . \quad$ CIB: $A 61 B 10 / 00$; A61B5/055; G01R33/48; G01R33/561; (IPC1-7): G01N27/00.

Stig Ljunggren. A simple graphical representation of fourier-based imaging methods. Journal of Magnetic Resonance (1969), 54(2):338-343, $1983 . \quad$ URL http://www.sciencedirect.com/science/article/pii/0022236483900604/pdf? md5 $=0234663$ e571096cf38b3ef ed3221bb6b\&pid=1-s2 .0-0022236483900604-main . pdf\&_valck=1.

P. Mansfield and P. K. Grannell. NMR 'diffraction' in solids? Journal of Physics C: Solid State Physics, 6(22):L422, November 1973. ISSN 0022-3719. doi: 10.1088/0022-3719/ 6/22/007. URL http://iopscience.iop.org/0022-3719/6/22/007.

S. Meiboom and D. Gill. Modified Spin-Echo method for measuring nuclear relaxation times. Review of Scientific Instruments, 29(8):688-691, August 1958. ISSN 0034-6748, 1089-7623. doi: 10.1063/1.1716296. URL http://scitation.aip.org/content/aip/ journal/rsi/29/8/10.1063/1.1716296.

Kevin W. Moser, L.Guy Raguin, Andrew Harris, H.Douglas Morris, John Georgiadis, Mark Shannon, and Mike Philpott. Visualization of Taylor-Couette and spiral poiseuille flows using a snapshot FLASH spatial tagging sequence. Magnetic Resonance Imaging, 18(2):199-207, February 2000. ISSN 0730-725X. doi: 10.1016/S0730-725X(99)00121-6. URL http://www.sciencedirect.com/science/article/pii/S0730725X99001216. 
David G. Norris, Peter Börnert, Torsten Reese, and Dieter Leibfritz. On the application of ultra-fast rare experiments. Magnetic Resonance in Medicine, 27(1):142-164, 1992. ISSN 1522-2594. doi: 10.1002/mrm.1910270114. URL http://onlinelibrary .wiley . com/doi/10.1002/mrm.1910270114/abstract.

E. M. Purcell, H. C. Torrey, and R. V. Pound. Resonance absorption by nuclear magnetic moments in a solid. Physical Review, 69(1-2):37-38, January 1946. doi: 10.1103/ PhysRev.69.37. URL http://link.aps.org/doi/10.1103/PhysRev.69.37.

M. Rokitta, U. Zimmermann, and A. Haase. Fast NMR flow measurements in plants using FLASH imaging. Journal of Magnetic Resonance, 137(1):29-32, March 1999. ISSN 1090-7807. doi: 10.1006/jmre.1998.1611. URL http://www.sciencedirect. com/science/article/pii/S1090780798916111.

Fritz Schick. SPLICE: sub-second diffusion-sensitive MR imaging using a modified fast spin-echo acquisition mode. Magnetic Resonance in Medicine, 38(4):638-644, 1997. ISSN 1522-2594. doi: 10.1002/mrm.1910380418. URL http://onlinelibrary •wiley . com/doi/10.1002/mrm.1910380418/abstract.

A.J. Sederman, M.D. Mantle, and L.F. Gladden. Single excitation multiple image RARE (SEMI-RARE): ultra-fast imaging of static and flowing systems. Journal of Magnetic Resonance, 161(1):15-24, March 2003. ISSN 1090-7807. doi: 10.1016/ S1090-7807(02)00141-6. URL http://www.sciencedirect.com/science/article/ pii/S1090780702001416.

Andrew J Sederman, Mick D Mantle, Craig Buckley, and Lynn F Gladden. MRI technique for measurement of velocity vectors, acceleration, and autocorrelation functions in turbulent flow. Journal of Magnetic Resonance, 166(2):182-189, February 2004. ISSN 1090-7807. doi: 10.1016/j.jmr.2003.10.016. URL http://www.sciencedirect.com/ science/article/pii/S1090780703003483.

G. Shiko, A.J. Sederman, and L.F. Gladden. MRI technique for the snapshot imaging of quantitative velocity maps using RARE. Journal of Magnetic Resonance, 216:183191, March 2012. ISSN 1090-7807. doi: 10.1016/j.jmr.2012.01.021. URL http://www . sciencedirect.com/science/article/pii/S1090780712000560.

E. O. Stejskal and J. E. Tanner. Spin diffusion measurements: Spin echoes in the presence of a Time-Dependent field gradient. The Journal of Chemical Physics, 42(1):288-292, 
January 1965. ISSN 0021-9606, 1089-7690. doi: 10.1063/1.1695690. URL http:// scitation.aip.org/content/aip/journal/jcp/42/1/10.1063/1.1695690.

Alexander B. Tayler, Martin Benning, Andrew J. Sederman, Daniel J. Holland, and Lynn F. Gladden. Ultrafast magnetic-resonance-imaging velocimetry of liquid-liquid systems: Overcoming chemical-shift artifacts using compressed sensing. Physical Review E, 89(6):063009, June 2014. doi: 10.1103/PhysRevE.89.063009. URL http: //link.aps.org/doi/10.1103/PhysRevE.89.063009.

Donald B. Twieg. The k-trajectory formulation of the NMR imaging process with applications in analysis and synthesis of imaging methods. Medical Physics, 10(5): 610-621, September 1983. ISSN 0094-2405. doi: 10.1118/1.595331. URL http: //scitation.aip.org/content/aapm/journal/medphys/10/5/10.1118/1.595331. 



\section{Rheology of Wormlike Micelles}

'Rheology' is defined as the study of the flow and deformation of matter (Barnes and Hutton (1989)). The term is derived from the Greek word 'rhéó', which means 'flow'. Following the very general definition of rheology, this research field would actually encompass all studies concerning the behaviour of matter. However, rheology is mainly focused on condensed matter that does not fit into the classical picture of solid and liquid. Complex fluids, for example, exhibit structures on the microscopic scale which in turn couple with their flow behaviour. As such, these fluids can attain a certain amount of elasticity, a property usually ascribed to solids. The study of complex fluids can be seen as one of the main exercises for rheologists. Rheometry refers to the experimental techniques and tools available for rheological studies. The tool that is most important here is the cylindrical Couette cell, where a fluid is sheared between two concentric cylinders. The circular nature of this flow will not only have implications on the flow of the studied fluid but also the NMR measurement, as shall be outlined during the course of this thesis.

Wormlike micellar (WLM) fluids are a unique class of complex fluids and of main interest for the present work. The microstructure of this material class is induced by a self-assembly process of surfactant molecules that form aggregates resembling wormy creatures. Due to their peculiar microstructure, wormlike micelles have unusual rheological properties, which have been intensively studied over the last 20 years (Berret (2006)). A particular flow effect called 'shear banding' is commonly found for wormlike micellar solutions and thoroughly investigated for the studies presented in this work. Furthermore, there is also a variety of industrial applications for wormlike micelles (Yang (2002)).

\subsection{Principles of Shear Rheology}

\subsubsection{Hookean Solid}

Hooke's law states that the force $F$ necessary to extend a spring by some distance $x$ is proportional to that distance. Similarly one can say that the degree of deformation experienced by a Hookean solid is in direct proportion with the force applied to it. For a 
convenient mathematical treatment, the force $F$ is normalized by the area $A$ to which it is applied to, yielding the stress $\sigma=F / A$. The deformation itself is described by the strain $\gamma$, which is a measure for the displacements within the body relative to some reference length. In Fig. 3.1 the top of a solid (Hookean) block is moved parallel by an amount $l$ relative to the bottom of the block resulting in a strain $\gamma=l / L$ with $L$ being the height of the block. This way of deforming the object is known as shearing. The elastic modulus $G$ is the proportionality constant relating the (shear) stress $\sigma$ and the strain $\gamma$ so that

$$
\sigma=G \gamma
$$

$G$ is the equivalent of the spring constant $k$ appearing in the familiar form of Hooke's law $F=k x$. Consequently if a constant shear stress is applied to the Hookean solid it deforms in a way such that the strain $\gamma=\sigma / G$. It remains in this configuration until the stress ceases, which causes the solid to return to its original form, a behaviour commonly referred to as elastic. In a way the Hookean solid remembers its past state.
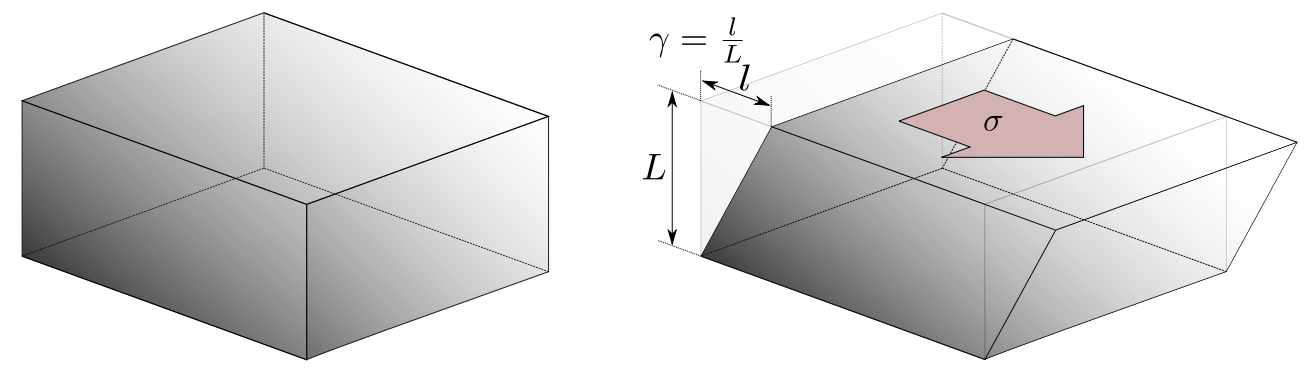

Fig. 3.1: The application of a constant shear stress $\sigma$ causes a deformation of the solid object such that $\sigma=l / L$. Upon stress cessation, the solid block returns to its original state.

\subsubsection{Newtonian Fluid}

The Newtonian fluid can be seen as the liquid counterpart of the Hookean solid. A shear motion can be exerted on the fluid by placing it between two parallel plates moving relative to each other (Fig. 3.2). The important quantity describing the fluid deformation, or more appropriately the fluid flow, is the strain rate $\dot{\gamma}$ (also called shear rate in the case of shearing). Generally defined as the time derivative of the shear $\gamma$, it is given by $\dot{\gamma}=V / d$ for two parallel plates with relative velocity $V$ that are separated by some distance $d$. $\dot{\gamma}$ can 
be understood as a velocity gradient describing how fast parts of the fluid move relative to each other. The stress necessary to maintain a certain shear rate is given by

$$
\sigma=\eta \dot{\gamma}
$$

with the proportionality constant $\eta$ known as viscosity. Applying a stress $\sigma$ causes the Newtonian fluid to deform continuously with the constant shear rate $\dot{\gamma}=\sigma / \eta$ and thereby attaining a strain $\gamma=\dot{\gamma} t$ after some time $t$. In contrast to the Hookean solid, however, this strain is not recovered after the stress has ceased. Therefore one can say that the Newtonian fluid does not have a memory of its shear history. This behaviour is commonly referred to as viscous. The resulting flow field of a Newtonian fluid under shear as in Fig. 3.2 probably meets the intuitive expectation for the flow of a liquid under such conditions. This is related to the fact that the most common fluid in daily life, water, can be nominally classed as a Newtonian fluid. However, there is a large class of other fluids termed non-Newtonian fluids or complex fluids exhibiting a more complicated flow behaviour. An example for an unusual flow behaviour of a non-Newtonian fluid under shear is given in section 3.4.

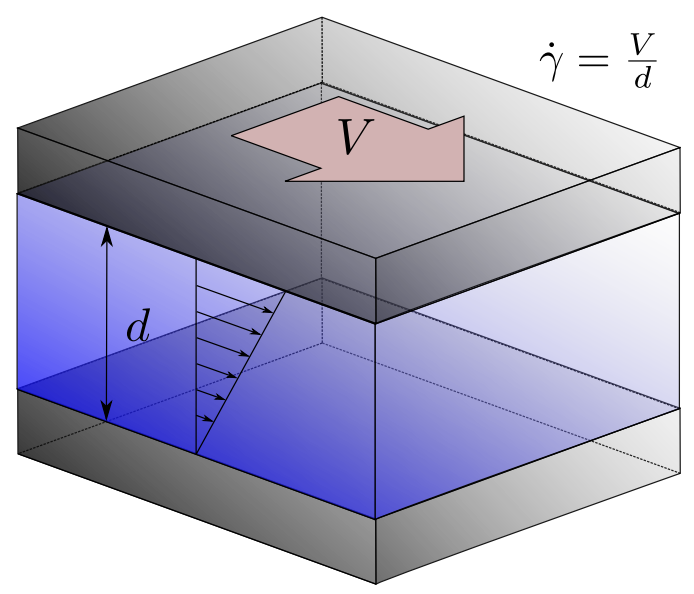

Fig. 3.2: A Newtonian liquid is sheared between two parallel planes with relative velocity $V$ that are separated by a distance $d$. The velocity gradient $\dot{\gamma}$, known as shear rate, is given by $\dot{\gamma}=V / d$ in this case.

Furthermore, it is common to differentiate between linear and nonlinear rheology. In linear rheology only small stresses or strains are considered, as for example encountered in oscillatory stress/strain experiments with small amplitudes. The term 'linear' stems from the fact that the response of the fluid (e.g. stress) is directly proportional to an input (e.g. strain) in that region (Barnes and Hutton (1989)). On the other hand, continuous sample 
deformation at a constant shear rate $\dot{\gamma}$, which is of main interest for the present work, can usually be considered as nonlinear deformation.

The situation where a fluid is sheared between two parallel plates as shown in Fig. 3.2 is known as simple shear. Also, it is worth noting that equations relating stress and strain/strain rate are called constitutive equations in the field of rheology. The constitutive equation for the Newtonian fluid $\sigma=\eta \dot{\gamma}$ (Eq. (3.2)) is probably the most simple relationship of that kind. For non-Newtonian fluids these equations can take an arbitrary amount of complexity depending on the fluid being modelled. Consequently, corresponding equations of motion are usually difficult to solve and require a high amount of sophistication in order to extract flow characteristics even in the case where inertia can be neglected.

\subsubsection{Maxwell model}

The first model for a viscoelastic fluid has been provided by Maxwell (1866), even though he actually intended to model the flow of gases. A viscoelastic fluid is a liquid that has both viscous and elastic properties (Bird and Hassager (1987)). The constitutive equation proposed by Maxwell has the following form:

$$
\sigma+\frac{\eta}{G} \frac{\partial \sigma}{\partial t}=\eta \dot{\gamma} .
$$

In case of a steady-state motion this equation simplifies to the Newtonian fluid with viscosity $\eta$ (Eq. (3.2)). For sudden changes in the stress on the other hand, the time derivative term dominates and the Hookean solid (Eq. (3.1)) with elastic modulus $G$ is recovered by integrating with respect to time. The term $\eta / G$ appearing in Eq. (3.3) is often called (stress) relaxation time and shall be denoted by $\lambda$. The choice of nomenclature becomes clear when considering the response of a Maxwell fluid to the following shear protocol. After shearing the fluid at all times $t<0$ with a constant shear rate $\dot{\gamma}_{0}$, the shear motion is stopped at $t=0$. Solving the differential equation Eq. (3.3) under these conditions leads to the following solution for $\sigma(t)$ at $t>0$ :

$$
\sigma(t)=\eta \dot{\gamma}_{0} \exp \left(-\frac{t}{\lambda}\right) .
$$

This means that the constant stress $\sigma_{0}=\eta \dot{\gamma}_{0}$ necessary to maintain the steady-state shear motion for $t<0$ does not immediately cease to zero after shearing is stopped. It rather 
relaxes exponentially with the time constant $\lambda$ governing this process. Likewise, if the temporal order of the shear protocol is reversed then the shear stress at $t>0$ is given by

$$
\sigma(t)=\eta \dot{\gamma}_{0}\left(1-\exp \left(-\frac{t}{\lambda}\right)\right)
$$

which means that the stress approaches the steady value $\sigma_{0}$ with some time constant $\lambda$.

While being the first model for a viscoelastic fluid, the Maxwell model can also be regarded as the simplest model for a fluid that has both properties of a solid and a liquid. Generally many viscoelastic fluids require more complex mathematical description. However, certain rheological properties of wormlike micellar solutions (discussed in section 3.3.2) in the linear regime are captured by the Maxwell model (Cates and Fielding (2006)). In particular, these solutions exhibit a monoexponential stress decay characterized by a single relaxation time $\lambda$ as encountered for the Maxwell fluid.

\subsubsection{Tensorial Character and Dimensionless Numbers}

So far, stresses and strains have been treated as scalar quantities. In general however stresses can act on all faces in arbitrary directions of the studied object. Referring a mathematical derivation to one of the textbooks (e.g. Bird and Hassager (1987)), it can be shown that stress, strain and strain rate can be locally described by a $3 \times 3$ tensor. Each component of the tensor is acting on a finite volume element in a manner as depicted in Fig. 3.3 for $\sigma$. Stresses acting parallel to the corresponding surface normal (e.g. $\sigma_{x x}$ ) are called 'normal stresses', while stresses acting perpendicular are known as 'shear stresses'. With regards to the shear geometry mainly used in this work, the cylindrical Couette cell (see section 3.2.1.1), a scalar representation for the stress and strain rate is sufficient in most cases. However, the spatial dependence of these quantities has to be taken into account. In particular, it shall be mentioned that the stress is constant throughout a Newtonian fluid in the simple shear condition, which is shown in Fig. 3.2. In contrast, this is not the case for the cylindrical Couette cell.

In fluid dynamics and rheology it is common to characterize the flow by relevant dimensionless numbers. The well-known Reynolds number Re is the ratio between inertial and viscous forces. A general definition can be given as

$$
\operatorname{Re}=\frac{\rho v L}{\eta}
$$

for a Newtonian fluid with viscosity $\eta$ and density $\rho$ (Bird and Hassager (1987)). $L$ is a characteristic length scale related to the flow situation and $v$ a reference velocity. For high 


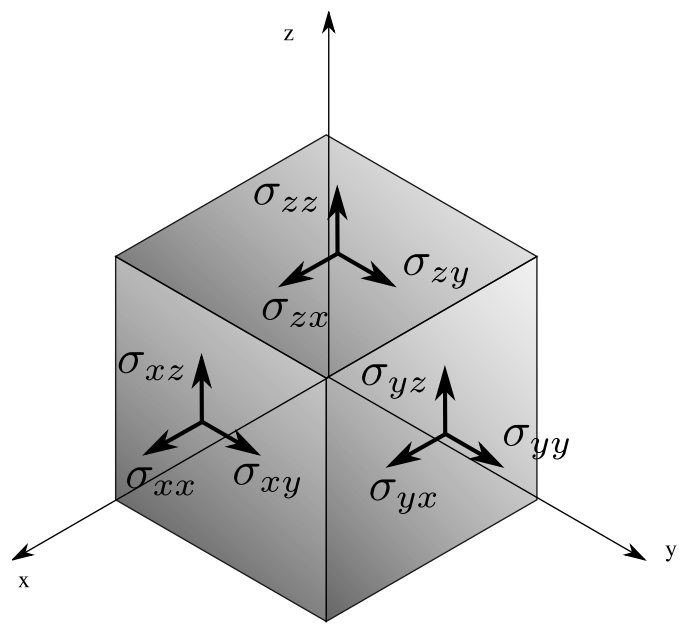

Fig. 3.3: Stress tensor acting on a finite volume element.

Reynolds numbers the flow is dominated by inertial forces, which can cause instabilities and turbulence. For low Reynolds numbers viscous forces are dominant resulting in a laminar flow pattern for Newtonian fluids. For the present study, it can be safely assumed that the flow is at low Reynolds numbers for all experiments. Therefore, inertial forces are not regarded as being of importance in the discussion of the flow phenomena outlined later. The dimensionless number being important for the flow of a viscoelastic material is the Weissenberg number Wi. It is the product of a characteristic time of the fluid and a characteristic rate of deformation (Dealy (2010)). For the case of shear flow it shall be defined as

$$
\mathrm{Wi}=\lambda_{c} \dot{\gamma}
$$

with $\dot{\gamma}$ as the shear rate and $\lambda_{c}$ being the characteristic relaxation time of the fluid. With regards to a Maxwellian type liquid, the characteristic relaxation time $\lambda_{c}=\lambda$. At high Weissenberg numbers the flow can become unstable and complex patterns might develop (see Muller (2008) and reference therein). This transition is usually accompanied by the emergence of secondary flows which disturb the base flow structure. For sufficiently high values of Wi the flow can even evolve towards a state that presents strong analogies with turbulence (Groisman and Steinberg (2000); Larson (2000)). Therefore one can say that the Weissenberg number takes the role of the Reynolds number for the viscoelastic fluid. Furthermore, it shall be noted that flow instabilities originating from the elasticity of a viscoelastic fluid are commonly referred to as elastic instabilities. 


\subsection{Rheometry}

\subsubsection{Shearing Geometries}

Most of the shear flow experiments presented in this thesis used a cylindrical Couette cell. Therefore its key aspects are described in the following section. Two other common shear geometries, namely the cone-and-plate and the plate-plate, shall be discussed briefly in the subsequent section.

\subsubsection{Cylindrical Couette Cell}

The cylindrical Couette cell consists of two concentric cylinders with differing radii. By rotating the cylinders relative to each other a shear motion is imposed on the fluid situated in the gap (see Fig. 3.4). The geometric simplicity of the shear cell has attracted the attention of scientists for centuries, maybe most prominently that of Newton in his Principia in 1687 (Newton, 1962, p.385-386). It is named after the French physicist Couette (1890) who studied it extensively at the end of the 19th century. In literature it is also known as Taylor-Couette (TC) cell, owing to the work by Taylor (1923) on flow instabilities at high Reynolds numbers in this cell. In his work, Taylor observed flow vortices stacked in the axial direction of the cell. Hence, this direction is also known as the 'vorticity direction'.

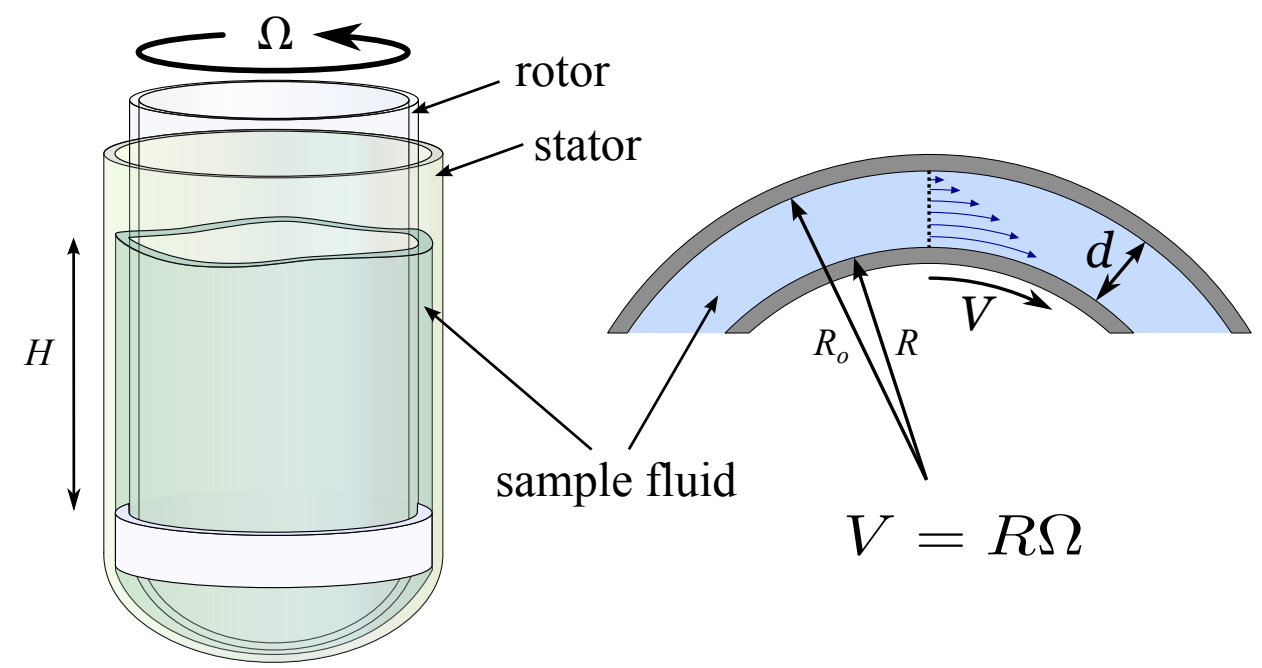

Fig. 3.4: Schematic of a cylindrical Couette cell consisting of two concentric cylinders. Here the inner cylinder (rotor) is rotated with an angular velocity of $\Omega$ while the outer cylinder is stationary (stator). This imposes a shear motion on the sample fluid which is situated in the gap between both cylinders. 
In a common setup the outer cylinder is kept stationary while the inner one is rotated with an angular velocity of $\Omega$. In this situation the two cylinders are called "stator" and "rotor" (see Fig. 3.4). The gap width is given by the difference in radii for both cylinders $d=R_{o}-R$. The stable base flow is of cylindrical nature and can be described as follows (Bird and Hassager (1987)):

$$
v_{\varphi}=v_{\varphi}(r), v_{r}=v_{z}=0 .
$$

The relevant shear rate component is given as:

$$
\dot{\gamma}_{r \varphi}(r)=-r \frac{\partial}{\partial r}\left(\frac{v_{\varphi}(r)}{r}\right)
$$

If a Newtonian fluid is placed in the cell shown in Fig. 3.4 then the following velocity profile is obtained:

$$
v_{\varphi}(r)=\frac{\Omega R^{2}}{R_{o}^{2}-R^{2}}\left(\frac{R_{o}^{2}}{r}-r\right) \quad \text { for } \quad r \in\left[R, R_{o}\right]
$$

Please note that the profile function fulfils the boundary conditions $v_{\varphi}(r=R)=R \Omega=V$ and $v_{\varphi}\left(r=R_{o}\right)=0$, also known as the no-slip boundary condition. Slip shall be briefly introduced in section 3.2.3. In Fig. 3.5 velocity profiles for two different values of $R$ and $d$ are shown. In the case where the geometrical ratio $\Lambda$, linked to the cell curvature, defined as

$$
\Lambda=d / R
$$

is small $(\Lambda=1 / 8$ for Fig. 3.5a), the deviations between a linear profile as expected for simple shear and the TC profile are small. For the $\Lambda=4 / 5$ case on the other hand, considerable deviations are observed (Fig. 3.5b). The case of $\Lambda \ll 1$ is known as the small gap limit in literature. In accordance with Fardin et al. (2012), the small gap condition $\Lambda \ll 1$ shall be satisfied for a geometry with dimensions as in Fig. 3.5a. A small value for $\Lambda$ also means that the local shear rate $\dot{\gamma}_{r \varphi}(r)$ (Eq. (3.9)) is close to the gap averaged or applied shear rate

$$
\dot{\gamma}_{a}=\frac{\Omega R}{d}
$$

over the whole gap range. In the case of an arbitrary value of $\Lambda$, however, $\dot{\gamma}_{r \varphi}(r) \propto 1 / r^{2}$. The same is true for the stress over the gap, i.e. $\sigma_{r \varphi} \propto 1 / r^{2}$. For a geometry with dimensions as in Fig. 3.5a, the relative stress difference between the inner and outer wall 
$\left(\sigma_{r \varphi}(R)-\sigma_{r \varphi}(R+d)\right) / \sigma_{r \varphi}(R)$ is about $20 \%$ as compared to $70 \%$ for Fig. 3.5b. In general, cylindrical Couette flow converges towards the simple shear case for $\Lambda \rightarrow 0$.
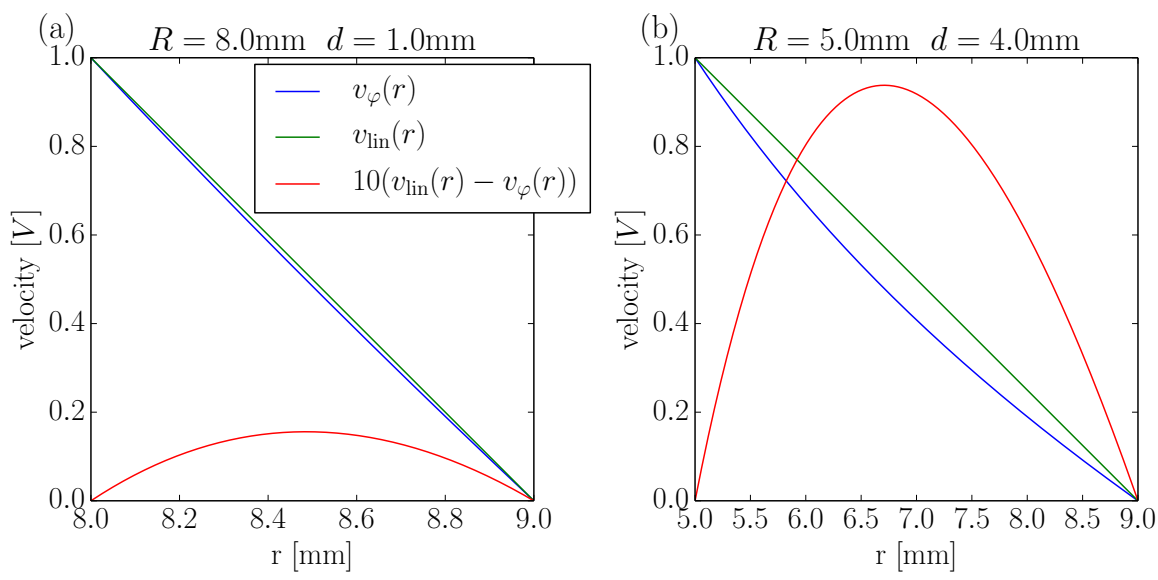

Fig. 3.5: Gap velocity profiles $v_{\varphi}(r)$ in the case of a Newtonian fluid for two cylindrical Couette cells with different radii $R$ and gap sizes $d$ (given in the plot titles). The velocity of the rotor is $V$. Also shown are linear profiles $v_{\operatorname{lin}}(r)$, which would be expected in the corresponding simple shear case, as well as the difference between $v_{\text {lin }}(r)$ and $v_{\varphi}(r)$ (multiplied by 10). For the cell with the smaller geometrical ratio $\Lambda$ (a), the velocity profile $v_{\varphi}(r)$ shows only slight deviations from the simple shear case whereas differences are considerable for the cell in (b). The plot legend applies to (a) and (b).

Finally it shall be pointed out that the equations presented in this section are strictly only true for infinite cylinders. In an experimental situation the flow geometry has a finite height. Furthermore, the fluid under investigation might not necessarily be filled up to the top of the Couette cell, as it will be described in section 5.1. Hence, $H$ shall denote the fill height of the fluid as indicated in Fig. 3.4. The influence of the top and bottom boundary condition is called end effects. How strongly the flow field is influenced by end-effects depends on the aspect ratio

$$
\Gamma=d / H
$$

For $\Gamma \ll 1$ end-effects are usually negligible, whereas a signature of the top and/or bottom boundary condition might be found in the flow field for higher values of $\Gamma$, in particular for regions closer to the respective boundaries. 


\subsubsection{Other Shear Geometries}

Cone-and-Plate In Fig. 3.6a a schematic of a cone-and-plate geometry is depicted. The fluid is trapped between a plate at the bottom and an inverted cone sitting on top of the plate. Usually the shear is exerted on the fluid by rotating the cone. Most notably, the cell allows for the measurement of normal stresses, while maintaining a constant gap averaged shear rate $\dot{\gamma}_{a}=\Omega / \tan \phi$, which is independent of the radial position. Similarly to the cylindrical Couette cell, the local shear rate and stress are not uniform in the gap direction. However, for typical cone angles $\phi$ of only a few degrees, the stress variance in the gap direction is only about $1 \%$, which is small compared to typical TC cells. Another advantage appreciated by rheologist is the small sample volume necessary to fill the gap between the plate and the cone. On the other hand, for techniques where the signal-tonoise ratio is proportional to the volume of the studied sample, as is the case for NMR, this might not be favourable. This is one of the reasons why mostly a TC cell has been used for the experiments here.

Plate-Plate The plate-plate geometry is depicted in Fig. 3.6b. The difference regarding the cone-and-plate geometry is that a parallel plate is used instead of an inverted cone at the top. In the case of Newtonian fluids, the shear stress and shear rate are uniform in the direction of the gap, whose size $d$ is defined by the distance between the two plates. However, stress and shear rate considerably vary in the radial direction. Therefore, this geometry is not considered for the present study.
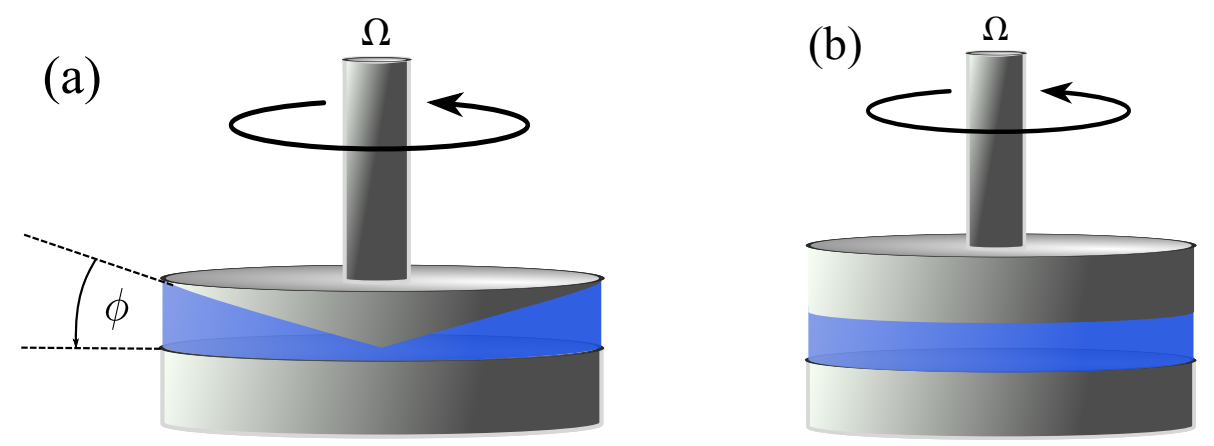

Fig. 3.6: Two shear geometries commonly used in rheology: (a) Cone-and-plate (b) Plateplate 


\subsubsection{Flow Curve}

Measuring the stress response $\sigma\left(\dot{\gamma}_{a}\right)$ of a fluid for a list of applied shear rates $\dot{\gamma}_{a}$ is a common procedure in rheometry. In doing so, one acquires a so-called flow curve, which is given by the relation of stress and shear rate for the fluid. In Fig. 3.7 schematic flow curves for commonly encountered fluid types are shown. Included are shear thinning and shear thickening fluids that for an increased rate of shear become less viscous and more viscous, respectively. Also depicted is the concept of a yield stress fluid, where a certain stress excess is needed in order to induce the flow of the material. Conventional rheometers allow the determination of shear stress by measuring the torque exerted on the shearing geometry. Stress measurement is not possible for Rheo-NMR equipment used here, as the high magnetic fields render precise torque measurement extremely difficult, though not impossible (see Grabowski and Schmidt (1994)).

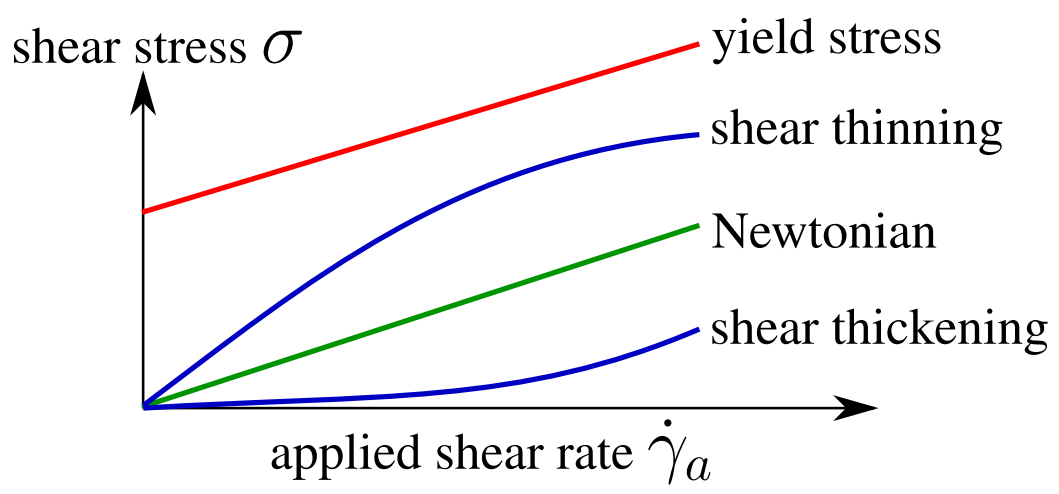

Fig. 3.7: Schematic flow curves for Newtonian and common non-Newtonian fluids.

\subsubsection{Slip}

A difficulty commonly encountered for rheology experiments is the presence of slip. With regards to the cylindrical Couette cell, slip is given by the mismatch of fluid velocities at the confining walls and the moving speed of the walls itself. Hence, the slip velocity $v_{s}$ at the rotor wall moving with $V$ is given by $v_{s}=V-v(0)$ with $v(0)$ being the local velocity of the fluid at the inner cylinder wall. The amount of slip can be reduced by altering the surface properties of the confining geometry. For example, a roughening of the rotor wall can reduce the slip velocity significantly for a wormlike micellar solution as will be discussed in section 6.3.1. 


\subsection{Surfactants and Wormlike Micelles}

\subsubsection{Surfactants in Solution}

Surfactants are amphiphilic molecules consisting of lyophilic (solvent loving) and lyophobic (solvent hating) parts. For water as the solvent, it is also common to use the terms hydrophilic (water loving) and hydrophobic (water hating). While it is energetically favourable for the (polar) hydrophilic section to be in contact with the water molecules, the hydrophobic part tries to avoid being in the vicinity of the latter due to its nonpolarity. Consequently, surfactants (a blend of 'surface acting agents') have a tendency to adsorb on surfaces in order to minimize solvent exposure of the hydrophobic component. Furthermore, they can self-organize in complicated structures depending on the chemical environment.

cetylpyridinium chloride
$(\mathrm{CPCl})$

Table 3.1: Two common surfactant molecules used for creating micellar solutions.

The hydrophobic part of the surfactant molecule is often given by a hydrocarbon chain of up to 20 carbon atoms and thus commonly referred to as the tail of the surfactant. In contrast, the hydrophilic section is called the head. Table 3.1 shows the chemical structure of two surfactant molecules that are of interest for the present thesis. Both surfactants have positively charged head groups and are therefore classed as cationic surfactants. Surfactants with a negative head group are called anionic surfactants and commonly used in detergents. Furthermore, there are also nonionic and zwitterionic surfactants. While the head group of the former bears no charge, two oppositely charged groups are contained in the head of the latter.

Due to their amphiphilic nature, these surfactant molecules can self-aggregate into a variety of structures. The simplest and probably best understood structure is the $m i$ celle. The underlying dynamics of the self-aggregation process are governed by the in- 
terplay of entropy and free energy of the solution. Below a concentration limit known as the critical micelle concentration (CMC) self-aggregation is hindered by entropic mixing forces and the solution consists mainly of isolated surfactant molecules, sometimes called unimers. For concentrations above the CMC, molecular aggregates start to form and grow in greater number while the unimer concentration stays more or less at the CMC (Israelachvili (2011)). Depending on the surfactant type, there are limits for the size of the aggregates. Therefore adding more surfactant above the CMC usually leads to a higher amount of micelles with a constant mean of the size distribution rather than a growth of the already existing ones (Israelachvili (2011)). This is in particular true for spherical micelles, which are discussed later in this section. However, wormlike micelles described in section 3.3.2 are an exception, as the size of these aggregates can very well depend on the surfactant concentration.

Temperature also plays an important role for the micellar formation. Below a certain temperature, which in turn depends on the surfactant concentration, the solute precipitates out of the solution as a hydrated crystal. Consequently at temperatures where the solubility limit lies below the CMC, the formation of micelles is not possible. The point where the solubility equals the critical micelle concentration is called the Krafft point. The corresponding temperature is known as the Krafft temperature. In Fig. 3.8 a schematic phase diagram for a surfactant solution is given.

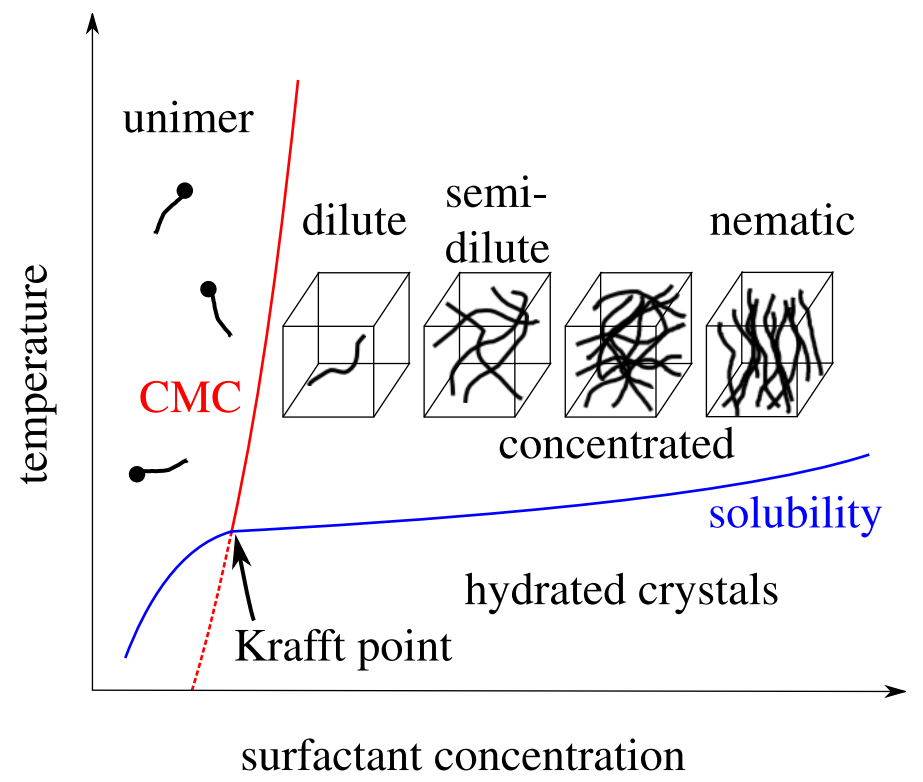

Fig. 3.8: Schematic phase diagram for a surfactant solution. The cubic boxes indicate the different concentration regimes in the case of a wormlike micellar solution. 
The structure of the surfactant aggregates can be anticipated, to a first order approximation, with the knowledge of only a few characteristics of the surfactant molecule (Israelachvili (2011)). Namely these are the volume of the hydrophobic tail $v$, the length of the hydrocarbon chain $l$ and the effective area of the head group $a$. The latter reflects the electrostatic interaction of the heads. Depending on the critical packing parameter $v / l a$, the aggregates can for example take the form of spheres, cylinders or planar layers. A pictorial interpretation is given in Fig. 3.9. The effective charge of the head group can be decreased by the addition of certain salts that screen the electrostatic repulsion of the former. Hence, the effective area $a$ is reduced, which in turn results in larger packing parameter.

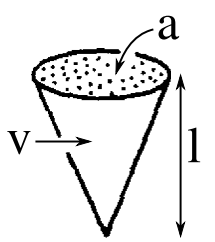

$\mathrm{v} / \mathrm{la}<1 / 3$

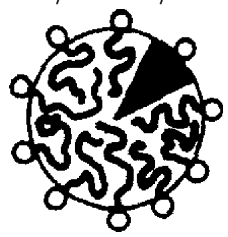

spherical

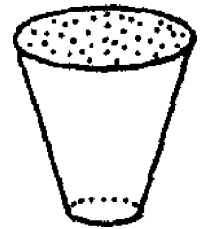

$1 / 3-1 / 2$

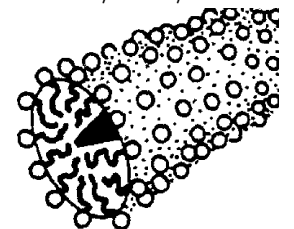

cylindrical

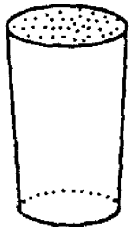

$1 / 2-1$

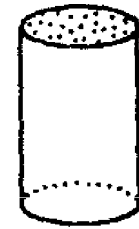

$\approx 1$

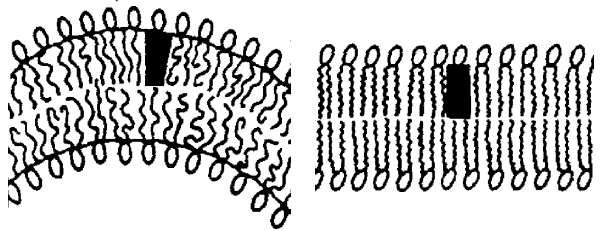

flexible

bilayer, vesicle

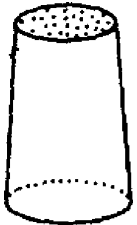

$>1$

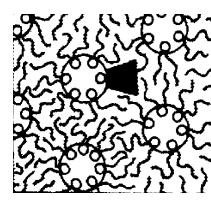

inverted

Fig. 3.9: Dependency of micellar structure on critical packing parameter (adapted from Larson (1999)).

Though the preceding treatment is a rough guide as to what micellar structure to except for certain surfactant type, it is incomplete as it neglects curvature effects of the self-assembled structure (Israelachvili (2011)). In particular for the wormlike aggregates discussed in the next section curvature effects play an important role in defining the shape of the micelle. As shall be outlined, wormlike micelles are flexible elongated structure and not stiff cylindrical rods as the packing parameter model might suggest.

\subsubsection{Wormlike Micelles}

The aggregates in certain cylindrical micellar solutions can be long elongated structures that exhibit considerable flexibility. These structures resemble wormy creatures and are hence called wormlike micelles. It has been observed that the average length of these 
wormlike structures can depend on the surfactant concentration even for a constant critical packing parameter. This is explained by the law of mass action, which favours longer wormlike aggregates (Cates and Fielding (2006)). The shape of a wormlike micelle can be fully described by only a few structural parameters, which can cover a broad range of length-scales (Dreiss (2007)). In Fig. 3.10 a schematic of a wormlike micellar aggregate is depicted along with the main dimensions of interest.

The overall length of the micelles is called the contour length $L$ and can vary from a few nanometres to microns. The average contour length $\bar{L}$ of certain wormlike micellar solutions can, for example, be predicted in terms of the surfactant volume fraction, the temperature and the end-cap energy required to form two hemispherical end-caps, by using a mean-field treatment (Dreiss (2007)). The second key structural parameter is the persistence length $l_{p}$ over which the micelles are considered to be rigid. Even though wormlike micelles can be extremely flexible, they can be regarded as stiff rods in smaller length scales (in the order of $l_{p}$ ) due to their large cross-section. It shall be noted that the persistance length is related to the Kuhn length $b$ known from polymer theory by $b=2 l_{p}$. In general, micellar flexibility plays an important role in the thermodynamics (phase behaviour) and dynamics (rheology) of the self-assembled system, and is of particular interest for the understanding of the isotropic-to-nematic phase as encountered in section 3.4. However, a more thorough analysis of theoretical models concerning phase transitions in wormlike micellar systems is beyond the scope of the thesis.

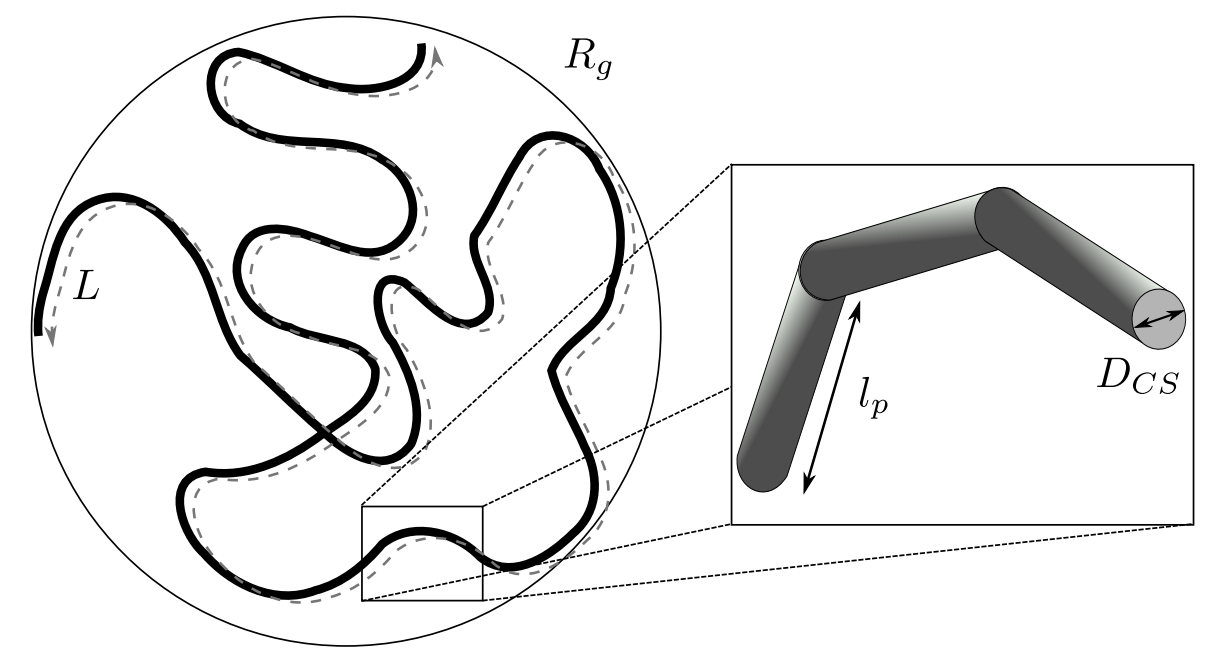

Fig. 3.10: Schematic representation of a wormlike micelle with characteristic length-scales: the radius of gyration $R_{g}$, the contour length $L$, the cross-section diameter $D_{C S}$ and the persistence length $l_{p}$. Adapted from Dreiss (2007). 
Apart from an increased average length at higher concentrations, the micelles also start forming an entangled network. This network adds a certain amount of elasticity to the solution, which is therefore classed as a viscoelastic fluid. At even higher concentrations the isotropic network becomes more ordered eventually forming a nematic phase. Different concentration regimes for a wormlike micellar solution are indicated in Fig. 3.8.

The structure of wormlike micelles is reminiscent of linear polymers. However, the bonds between individual segments of the micellar aggregate are not as strong as the covalent bonds between the monomers in a polymer. Consequently wormlike micelles break and recombine continually as opposed to polymeric molecules, which have a fixed length. Hence, wormlike micelles are also known as living polymers. These active dynamics give rise to the unique rheological properties of certain wormlike micellar solutions, most notably the monoexponential stress decay mentioned earlier in the context of the Maxwell model. For polydisperse polymer solutions a broad spectrum of stress relaxation times is encountered. In the framework of the reptation model (Gennes (1979)) this can be roughly explained by the fact that longer polymer chains relax slower upon sudden changes of either shear rate or stress, a process governed by the reptation time $\tau_{\text {rep }}$. Wormlike micelles on the other hand sample a huge range of chain lengths during the relaxation process, if the breakage and recombination process is much faster than the relaxation by reptation (snakelike motion), as explained by Cates (1987). Due to this sampling all micelles effectively relax at the same rate. Consequently, the wormlike micellar solution only exhibits a single stress relaxation time $\lambda$ in the linear regime. This remarkable analogy to the Maxwell fluid was pointed out in particular by Rehage and Hoffmann (1991), who regarded wormlike micelles as an ideal "model system for rheological research".

For the present work, the majority of experiments was undertaken on a wormlike micellar solution formed by cetylpyridinium chloride $(\mathrm{CPCl}$ ) (see Table 3.1 ) and an additional salt, namely sodium salicylate (NaSal), in the semidilute concentration regime (see Fig. 3.8). The solution is described in more detail in section 6.1. Berret et al. (1993) have shown that this solution behaves almost exactly like a Maxwell fluid in the linear regime. The response to constant shearing in the nonlinear regime (Berret et al. (1994)), however, reveals a relation between the applied shear rate $\dot{\gamma}_{a}$ and the observed stress not covered by the Maxwell model. This observation is ascribed to an effect called shear banding, which is discussed in the next section. 


\subsection{Shear Banding in Wormlike Micelles}

The phenomena of shear banding exhibited by wormlike micellar solutions is characterized by a partitioning of the sheared fluid in two or more regions of differing viscosities at nominally constant stress (Berret (2006)). The effect is usually associated with a stress plateau in the flow curve. The plateauing of the stress can be explained by a nonmonotonic region in the underlying constitutive relation (Fig. 3.11a) following a model suggested by Cates et al. (1993). In the framework of this model, the fluid subdivides into two coexisting bands with local shear rates $\dot{\gamma}_{1}$ and $\dot{\gamma}_{2}$ given by the beginning and the end of the stress plateau (Fig. 3.11b). The proportions of each band are often described by a simple lever rule

$$
\dot{\gamma}_{a}=(1-\alpha) \dot{\gamma}_{1}+\alpha \dot{\gamma}_{2}
$$

where $\alpha$ is the proportion of the band with higher local shear rate $\dot{\gamma}_{2}$ and lower viscosity. An increase of the applied shear rate within the stress plateau leads therefore to an increase in $\alpha$ while maintaining a constant shear stress. It shall be pointed out that there exists no preferred site for the nucleation of the high shear rate band in the simple shear case. Even the existence of multiple low and high shear rate bands would be possible. In the case of cylindrical Couette flow on the other hand, the high shear rate band preferably develops at the inner wall where shear stress is higher (Salmon et al. (2003)).

First measurements indicating the existence of a banded structure were done with optical techniques (Decruppe et al. (1995); Makhloufi et al. (1995)). The emergence of a low viscosity band is usually linked to an isotropic-to-nematic transition in the high shear rate region. The alignment of the wormlike micelles in the flow direction reduces the drag, causing the observation of a reduced viscosity. These structural changes also alter the optical properties and hence make it possible to discern different bands with optical methods. However, the first direct detection of shear banding, i.e. a measurement of flow velocity for a sheared fluid in the banded state, came from NMR velocimetry (Britton and Callaghan (1997)). The simple lever rule scenario was roughly confirmed by, for example, Salmon et al. (2003), but a range of instabilities in the structure of the banded flow have been observed recently (see Fardin and Lerouge (2012) and references therein). These instabilities shall be discussed in chapter 6 . 
(a)

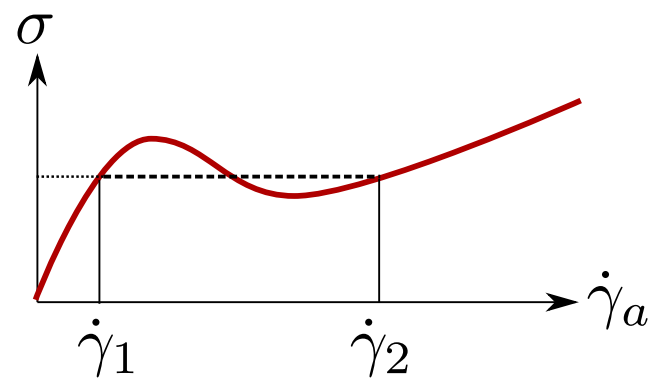

(b)

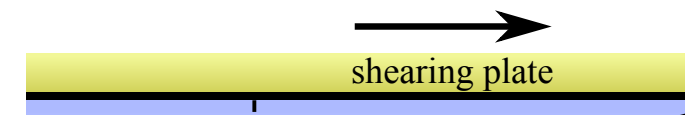

nematic

isotropic

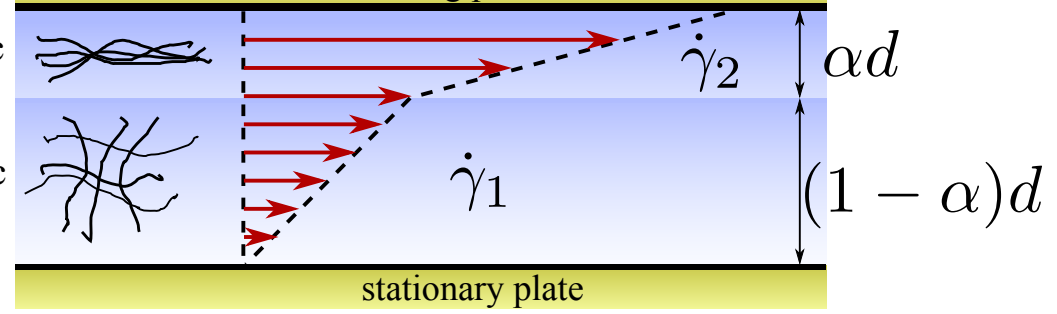

Fig. 3.11: (a) Schematic of the underlying constitutive relation (solid line) and the observed flow curve (dashed where different) for a shear banding micellar solution. The shear rates $\dot{\gamma}_{1}$ and $\dot{\gamma}_{2}$ mark the beginning and the end of the stress plateau. (b) The expected flow field for a shear banding solution following the simple lever rule (Eq. (3.14)) at an applied shear rate $\dot{\gamma}_{a}$ within the stress plateau. The emergence of a low viscosity band is associated with a phase change from an isotropic to a nematic state. $d$ is the distance between the plates.

\section{Bibliography}

Howard A. Barnes and John Fletcher Hutton. An Introduction to Rheology. Elsevier, 1989. ISBN 9780444871404.

Jean-François Berret. Rheology of wormlike micelles: Equilibrium properties and shear banding transitions. In Richard G. Weiss and Pierre Terech, editors, Molecular Gels, pages 667-720. Springer Netherlands, January 2006. ISBN 978-1-4020-3352-0, 978-14020-3689-7. URL http://link. springer.com/chapter/10.1007/1-4020-3689-2_ 20.

Jean-François Berret, Denis C. Roux, and Gregoire Porte. Isotropic-to-nematic transition in wormlike micelles under shear. Journal de Physique II, 4(8): 1261-1279, August 1994. ISSN 1155-4312, 1286-4870. doi: 10.1051/jp2: 1994198. URL http://jp2.journaldephysique.org/articles/jp2/abs/1994/08/ jp2v4p1261/jp2v4p1261.html. 
Jean Francois Berret, Jacqueline Appell, and Gregoire Porte. Linear rheology of entangled wormlike micelles. Langmuir, 9(11):2851-2854, November 1993. ISSN 0743-7463. doi: 10.1021/la00035a021. URL http://dx.doi.org/10.1021/la00035a021.

R.B. Bird and O. Hassager. Dynamics of Polymeric Liquids: Fluid mechanics. Dynamics of Polymeric Liquids. Wiley, 1987. URL http://books.google.co.nz/books?id= posvAQAAIAAJ.

Melanie M. Britton and Paul T. Callaghan. Two-Phase shear band structures at uniform stress. Phys. Rev. Lett., 78(26):4930-4933, June 1997. doi: 10.1103/PhysRevLett.78. 4930. URL http://link.aps.org/doi/10.1103/PhysRevLett.78.4930.

M. E. Cates. Reptation of living polymers: dynamics of entangled polymers in the presence of reversible chain-scission reactions. Macromolecules, 20(9):2289-2296, September 1987. ISSN 0024-9297. doi: 10.1021/ma00175a038. URL http://dx.doi.org/10. 1021/ma00175a038.

M. E. Cates and S. M. Fielding. Rheology of giant micelles. Advances in Physics, 55 (7-8):799-879, 2006. ISSN 0001-8732. doi: 10.1080/00018730601082029. URL http: //dx.doi.org/10.1080/00018730601082029.

M. E. Cates, T. C. B. McLeish, and G. Marrucci. The rheology of entangled polymers at very high shear rates. EPL (Europhysics Letters), 21(4):451, 1993. URL http: //stacks. iop.org/0295-5075/21/i=4/a=012.

Maurice Couette. Études sur le frottement des liquides, par M. Maurice Couette. GauthierVillars et fils, 1890.

John M. Dealy. Weissenberg and deborah numbers - their definition and use. Rheolgy Bulletin, 79(2):14-18, July 2010. URL http://www.rheology.org/sor/publications/ rheology_b/RB2010Jul.pdf.

J. P. Decruppe, R. Cressely, R. Makhloufi, and E. Cappelaere. Flow birefringence experiments showing a shear-banding structure in a CTAB solution. Colloid and Polymer Science, 273(4):346-351, April 1995. ISSN 0303-402X, 1435-1536. doi: 10.1007/ BF00652348. URL http://link.springer.com/article/10.1007/BF00652348.

Cécile A. Dreiss. Wormlike micelles: where do we stand? recent developments, linear rheology and scattering techniques. Soft Matter, 3(8):956-970, July 2007. ISSN 1744-6848. 
doi: 10.1039/B705775J. URL http://pubs.rsc.org/en/content/articlelanding/ $2007 / \mathrm{sm} / \mathrm{b} 705775 \mathrm{j}$.

M.-A. Fardin and S. Lerouge. Instabilities in wormlike micelle systems. The European Physical Journal E, 35(9):1-29, September 2012. ISSN 1292-8941, 1292-895X. doi: 10. 1140/epje/i2012-12091-0. URL http://link. springer.com/article/10.1140/epje/ i2012-12091-0.

M. A. Fardin, T. J. Ober, V. Grenard, T. Divoux, S. Manneville, G. H. McKinley, and S. Lerouge. Interplay between elastic instabilities and shear-banding: three categories of Taylor-Couette flows and beyond. Soft Matter, 8(39):10072-10089, September 2012. ISSN 1744-6848. doi: 10.1039/C2SM26313K. URL http://pubs.rsc.org/en/ content/articlelanding/2012/sm/c2sm26313k.

Pierre-Gilles de Gennes. Scaling Concepts in Polymer Physics. Cornell University Press, 1979. ISBN 080141203X.

D. A. Grabowski and C. Schmidt. Simultaneous measurement of shear viscosity and director orientation of a Side-Chain Liquid-Crystalline polymer by Rheo-NMR. Macromolecules, 27(9):2632-2634, April 1994. ISSN 0024-9297. doi: 10.1021/ma00087a037. URL http://dx.doi.org/10.1021/ma00087a037.

A. Groisman and V. Steinberg. Elastic turbulence in a polymer solution flow. Nature, 405(6782):53-55, May 2000. ISSN 0028-0836. doi: 10.1038/35011019. URL http: //www. nature.com/nature/journal/v405/n6782/full/405053a0.html.

Jacob N. Israelachvili. Intermolecular and Surface Forces: Revised Third Edition. Academic Press, July 2011. ISBN 9780123919335.

R.G. Larson. The Structure and Rheology of Complex Fluids. Topics in Chemical Engineering. Oxford University Press, 1999. ISBN 9780195121971. URL http://books . google.co.nz/books?id=Vt9fw_pf1LUC.

Ronald G. Larson. Fluid dynamics: Turbulence without inertia. Nature, 405(6782):27-28, May 2000. ISSN 0028-0836. doi: 10.1038/35011172. URL http://www.nature.com/ nature/journal/v405/n6782/full/405027a0.html.

R. Makhloufi, J. P. Decruppe, A. Aït-Ali, and R. Cressely. Rheo-Optical study of wormlike micelles undergoing a shear banding flow. EPL (Europhysics Letters), 32(3):253, 1995. URL http://stacks.iop.org/0295-5075/32/i=3/a=011. 
J. C. Maxwell. On the dynamical theory of gases. Proceedings of the Royal Society of London, 15(0):167-171, January 1866. ISSN 0370-1662. doi: 10.1098/rspl.1866.0039. URL http://rspl.royalsocietypublishing.org/cgi/doi/10.1098/rspl.1866.0039.

Susan J. Muller. Elastically-influenced instabilities in Taylor-Couette and other flows with curved streamlines: a review. Korea-Aust. Rheol. J, 20(3):117-125, 2008. URL http://infosys.korea.ac.kr/PDF/KARJ/KR20/KR20-3-0117.pdf.

Isaac Newton. Mathematical Principles of Natural Philosophy and His System of the World: The Motions of Bodies. University of California Press, 1962. ISBN 9780520009288.

H. Rehage and H. Hoffmann. Viscoelastic surfactant solutions: model systems for rheological research. Molecular Physics, 74(5):933-973, 1991. ISSN 0026-8976. doi: 10.1080/00268979100102721. URL http://www.tandfonline.com/doi/abs/10.1080/ 00268979100102721.

Jean-Baptiste Salmon, Annie Colin, Sébastien Manneville, and François Molino. Velocity profiles in Shear-Banding wormlike micelles. Physical Review Letters, 90(22):228303, June 2003. doi: 10.1103/PhysRevLett.90.228303. URL http://link.aps.org/doi/ 10.1103/PhysRevLett.90.228303.

G. I. Taylor. Stability of a viscous liquid contained between two rotating cylinders. Philosophical Transactions of the Royal Society A: Mathematical, Physical and Engineering Sciences, 223(605-615):289-343, January 1923. ISSN 1364-503X, 1471-2962. doi: 10. 1098/rsta.1923.0008. URL http://adsabs .harvard.edu/abs/1923RSPTA .223 . 289T.

Jiang Yang. Viscoelastic wormlike micelles and their applications. Current Opinion in Colloid $\mathcal{E}$ Interface Science, 7(5-6):276-281, November 2002. ISSN 13590294. doi: 10.1016/S1359-0294(02)00071-7. URL http://www.sciencedirect.com/ science/article/pii/S1359029402000717. 



\section{Simulation of NMR Signal}

With the advent of computer technology and a wider accessibility of high performance computational devices, numerical simulations of NMR experiments have developed into a useful tool in understanding underlying mechanisms. There is a variety of programs available that cover NMR spectroscopy (e.g. Veshtort and Griffin (2006)), solid-state NMR (see Edén (2003)) and magnetic resonance imaging (e.g. Stöcker et al. (2010)). While quantum mechanical approaches are mainly used in the first two cases, a semi-classical description by the Bloch equations (Eq. (2.5)) is common for simulating MRI experiments. For the present work, two different simulations based on the Bloch equations have been developed. The first called 'velosim' allows for the numerical computation of the NMR signal generated by a PGSE-RARE sequence (described in section 5.2.3.1) in the case where the shear flow of a sample fluid in a cylindrical Couette geometry is imaged. The second ('SliceSelection') is focused on the evolution of the magnetization under a slice selective pulse. Both programs are used in chapter 5 to discuss and explain artefacts observed in the images and velocity maps generated by the PGSE-RARE pulse sequence used for the experiments. The simulation programs were written in Python and make strong use of the SciPy (van der Walt et al. (2011)) and matplotlib (Hunter (2007)) packages. Furthermore, parallelization of the problem allows for a fast computation of results for both simulations.

\subsection{RARE sequence simulation}

The main motivation behind the 'velosim' simulation was the study of motion related artefacts in velocity maps obtained with a PGSE-RARE sequence in the case of cylindrical Couette flow with particular focus on shear banding. Even though the program is optimized for the aforementioned situation, other imaging pulse sequences (e.g EPI) or flow types (e.g. Poiseuille flow) can be implemented within the framework of the simulation. In the present section the general operation of the program shall be described. In section 5.2.3 the simulation program is used to study the shear flow in a specific Couette geometry. 
Initialization At the beginning of the simulation procedure the input geometry is rasterized into a three dimensional (3D) grid. As the main focus was lying on Taylor-Couette cells, cylindrical coordinates are used for this grid. In order to keep the computational effort low, the grid is reduced to the region where the generation of coherent NMR signal is expected during the execution of the pulse sequence. This region shall be called active volume'. The dimensions of the active volume in the radial direction are given by two values $r_{\text {in }}<r_{\text {out }}$, which usually correspond to the inner and outer radius of the Couette cell. In the axial direction, the range of the active volume is defined by $[-H / 2, H / 2]$, where $H$ is the height of the geometry. In the angular direction two cases have to be distinguished, as the selection of a centred slice oriented in the $x z$-plane with thickness $s$ is possible. The orientation of the coordinate system and other variables are defined in Fig. 4.1. In the case where $s>r_{\text {out }}$, the angular range $\Delta \varphi$ is simply $[0,2 \pi]$. Otherwise, the angular range is depending on the radial position, as shown in Fig. 4.1a. Please note that in a real NMR experiment (as opposed to an NMR simulation experiment) the active volume is usually given by the region that is coherently excited by selective pulses (see section 2.1.9). These pulses are however not available for the simulation due to high demands in computational power (see also section 4.2). Therefore, the reduction of the grid is also a means of implicitly including the effect of selective excitation.
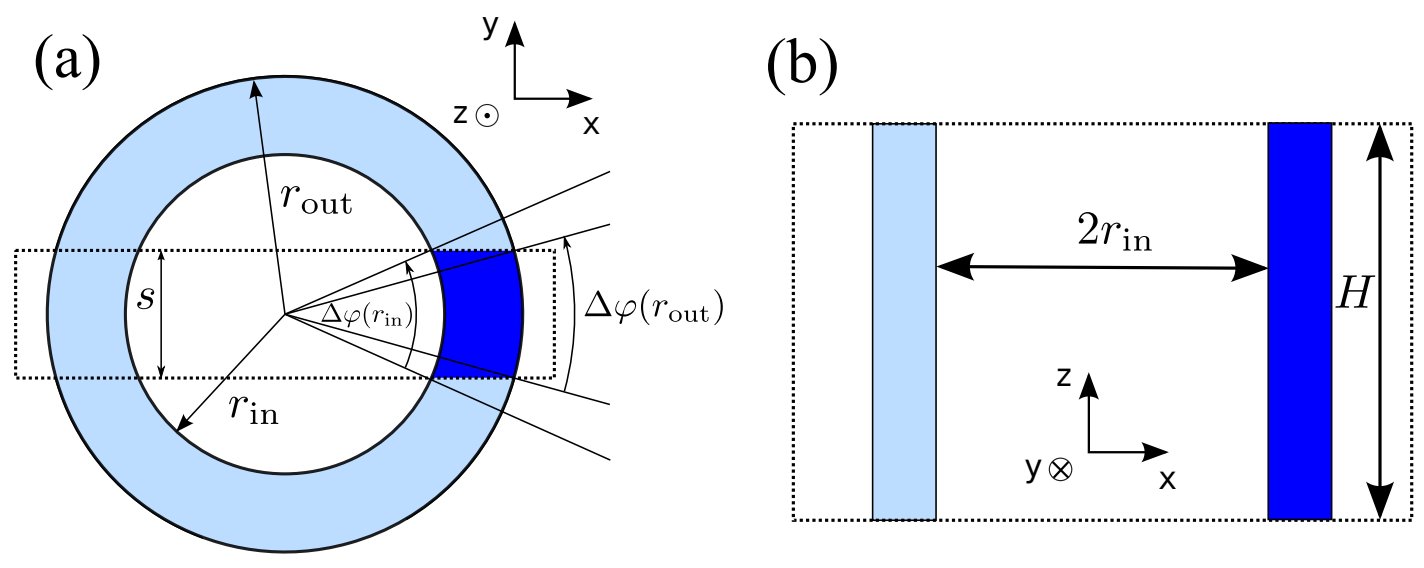

Fig. 4.1: Horizontal (a) and vertical (b) cuts through the input geometry for the simulation program. The dark blue sections mark the active volume. Dashed lines indicate the slice. Please note that for slice thicknesses $s<r_{2}$, the angle range $\Delta \varphi$ used for the rasterization is depending on the radial position $r$.

The number of points in the active volume grid can be chosen individually in the radial, angular and axial direction, denoted by $N_{r}, N_{\varphi}$ and $N_{z}$, respectively. Points are spaced evenly over the ranges in each direction with $N=N_{r} N_{\varphi} N_{z}$ being the total number of 
points in the grid. Each point in the grid is described by a vector $\mathbf{p}_{j}=\left(r_{j}, \varphi_{j}, z_{j}\right)$ with $j=1 \ldots N$. In Fig. 4.2 a horizontal cut through the rasterized (initial) active volume for $s<r_{\text {out }}$ case is shown in conjunction with the graphical user interface (GUI) of the program. Furthermore, it shall be noted that the geometry is assumed to be in the initial state at $t=0$. However, the start of the pulse sequence does not necessarily have to coincide with $t=0$.

After the geometry is set up, a magnetization vector $\mathbf{m}_{j}$ is assigned to each grid point $j$. $\mathbf{m}_{j}$ is represented by a vector $\left(m_{x y, j}, m_{z, j}\right)$ where $m_{x y, j}$ denotes the (local) transverse magnetization in complex notation. $m_{z, j}$ is the magnetization component in the axial direction. In the initial equilibrium configuration no transverse magnetization is present, i.e. $m_{x y, j}=0 . m_{z, j}$ on the other hand is scaled by the area of the corresponding grid voxel defined by $\left(\left[r_{j}, r_{j}+\mathrm{d} r\right],\left[\varphi_{j}, \varphi_{j}+\mathrm{d} \varphi\right]\right) . \quad \mathrm{d} r$ and $\mathrm{d} \varphi$ are the stepsizes in the radial and angular direction as defined in Fig. 4.3a. The scaling ensures a constant spin density over the whole active volume.

Pulse Sequence In order to study the evolution of the spin system under a pulse sequence, the Bloch equations in the on-resonant rotating frame are solved for each grid point individually. In particular, this means that there is only one Larmor frequency present and there are no interactions between the magnetization vectors of individual grid points. Spin relaxation effects, such as $T_{1}$ or $T_{2}$ relaxation, are not implemented at the current stage. In the simulation program, a pulse sequence is an ordered list consisting of the following three elements: delays, rf (hard) pulses and gradient pulses. The elements are executed consecutively starting at a time $t=t_{0}$. Under a delay the spin system is static and only $t$ is advanced by the length of the delay. An rf pulse rotates the magnetization vector $\mathbf{m}_{j}$ by an angle $\theta$ around the $x$ or $y$-axis of the rotating frame. Rf pulses do not have a duration in the simulation program and are therefore applied instantaneously. For the execution of a gradient pulse the spatial position of each grid point is important. In order to account for this the grid point position is updated once per gradient pulse with respect to a reference time $t_{\text {ref }}$ before the effect of the gradient is applied on magnetization vector. The reference time is given by the temporal centre of the gradient pulse. For example, a gradient pulse of duration $\Delta t$ applied at time $t_{g}$ leads to a reference time $t_{\text {ref }}=t_{g}+\Delta t / 2$. In the case of purely tangential flow with angular velocity $\omega(r)$ the grid vectors $\mathbf{p}_{j}$ are then updated to $\left(r_{j}, \varphi_{j}+\omega\left(r_{j}\right) t_{\text {ref }}, z_{j}\right)$. The evolution of the transverse magnetization for a grid point $j$ under the influence of a gradient $\mathbf{G}=\left(G_{x}, G_{y}, G_{z}\right)$ over the period $\Delta t$ is then given by

$$
m_{x y, j}\left(t_{g}+t\right)=m_{x y, j}\left(t_{g}\right) e^{i \gamma \mathbf{r} \mathbf{G} t}
$$




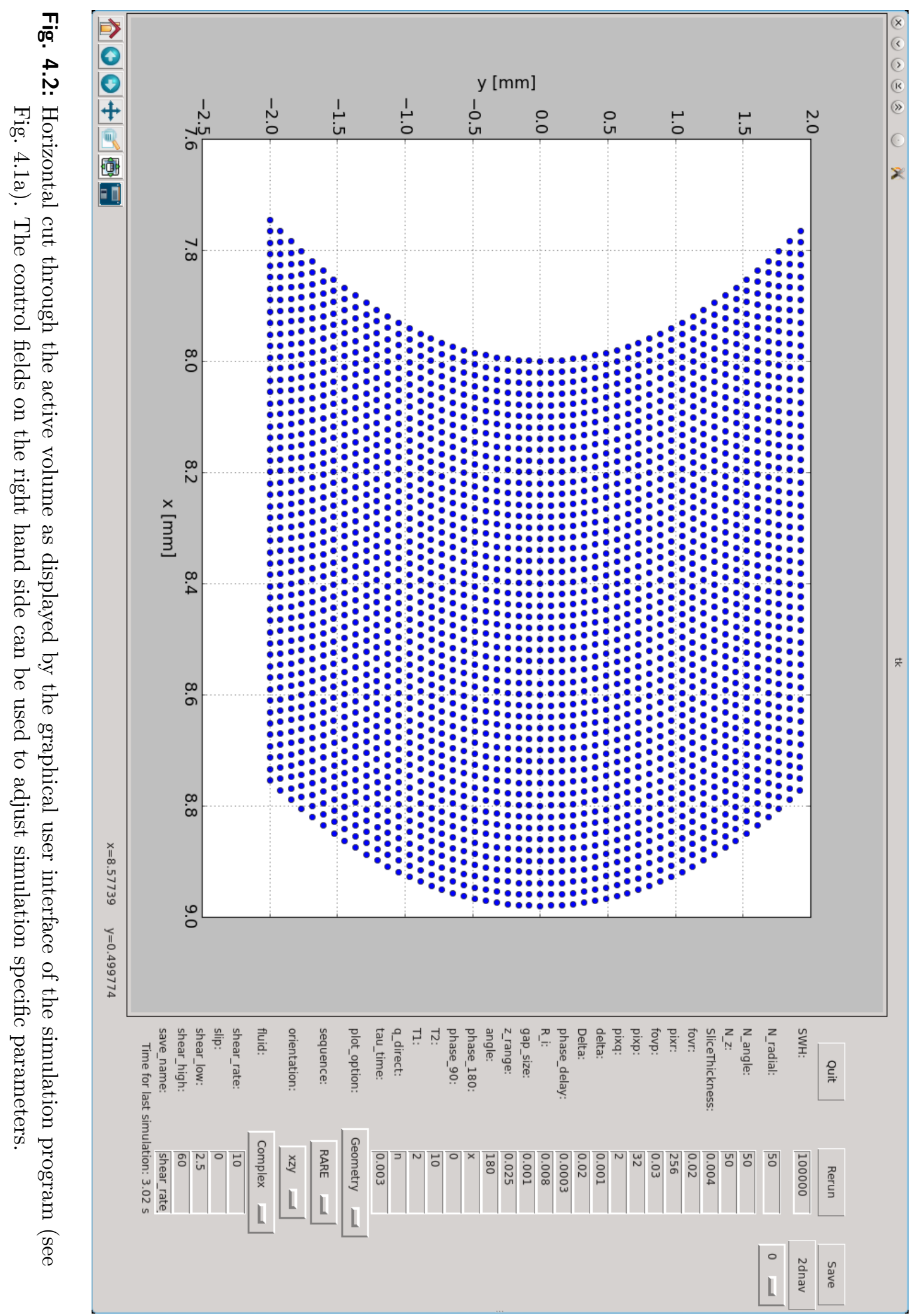


(a)

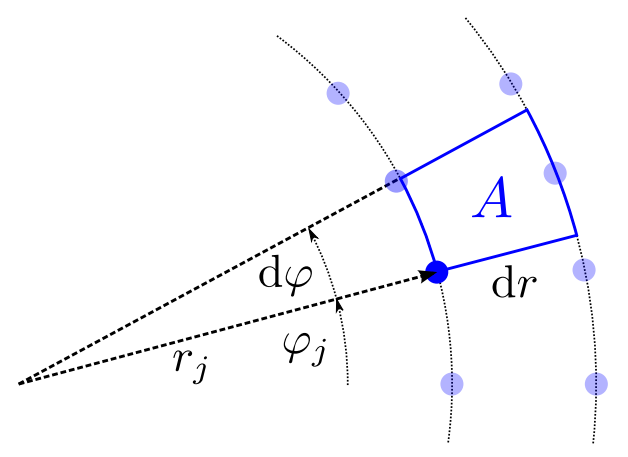

(b)

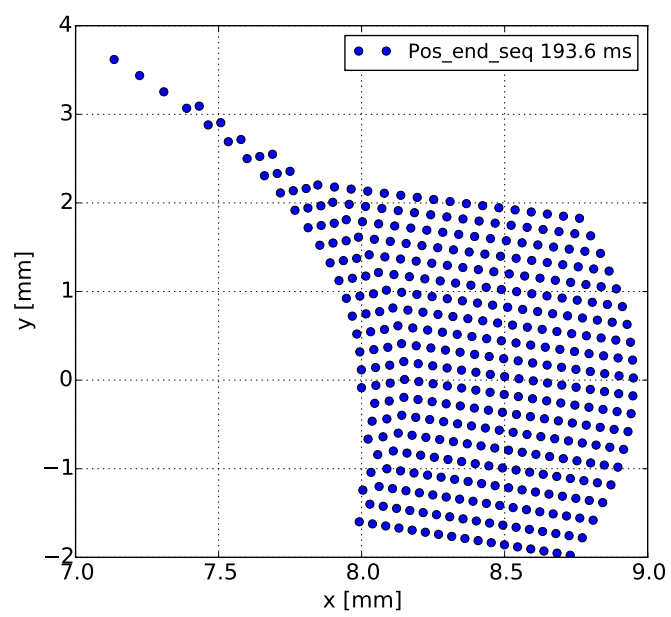

Fig. 4.3: (a) Close-up on grid point $j . A$ is the area of the corresponding area element, whereas $\mathrm{d} r$ and $\mathrm{d} \varphi$ are the stepsizes in the cylindrical grid. (b) Deformed grid in accordance to the (purely tangential) flow of the simulated fluid. Each point in the grid is treated as an isochromat under the application of a gradient pulse. Please note, that the grid density is lower as compared to Fig. 4.1 for better visualization.

with $\mathbf{r}=\left(r_{j} \cos \varphi_{j}, r_{j} \sin \varphi_{j}, z_{j}\right)$ and $t \in[0, \Delta t]$. This means that each grid point is treated as an isochromat. For a sufficiently dense grid it is assumed that the numerical descriptions of the active volume converges towards that of a continuum representation. Experimental complications such as eddy currents in the gradient coils or the necessity of a gradient ramp time are not considered in the simulation.

The flow profile in the radial direction at an applied shear rate $\dot{\gamma}_{a}$ is chosen to be linear or to follow the simple lever rule given in Eq. (3.14). For the latter the values $\dot{\gamma}_{1}$ and $\dot{\gamma}_{2}$ can be set individually. The fluid is assumed to be stationary at $r=r_{\text {out }}$ corresponding to the outer wall of the simulated Couette cell, whereas a velocity $V=\dot{\gamma}_{a} d$ is imposed at the inner wall $\left(r=r_{\text {in }}\right)$. Furthermore it is possible to include a slip velocity $v_{s}$ at the inner cylinder wall. Fig. $4.3 \mathrm{~b}$ shows the deformed grid during the execution of the pulse sequence in the case of a shear banding fluid. 
At any time $t$ during the pulse sequence the NMR signal can be recorded. In accordance with Eq. (2.9), the complex signal is given by the total transverse magnetization, which is obtained by summing over all grid points:

$$
S(t)=\sum_{j=1}^{N} m_{x y, j}(t) .
$$

The sampled signal for a 2D RARE-type sequence is shown in Fig. 4.4a. By means of Fourier transform a 2D image of the active volume can be generated depicted in Fig. 4.4b. Convergence of the simulated NMR signal for a certain parameter set can be tested by increasing the density of the simulation grid, e.g. by doubling the amount of grid points. If only marginal changes in the simulated signal are observed, it is assumed that the method has converged. Otherwise the grid density has to be increased in order to ensure convergence. If a series of simulation experiments is run, where, for example, only the applied shear rate is changed, convergence is typically only ensured for a single parameter set out of this series. Hence, it is assumed that the grid is sufficiently dense so that the simulated signal for all other experiments does also converge.

(a)

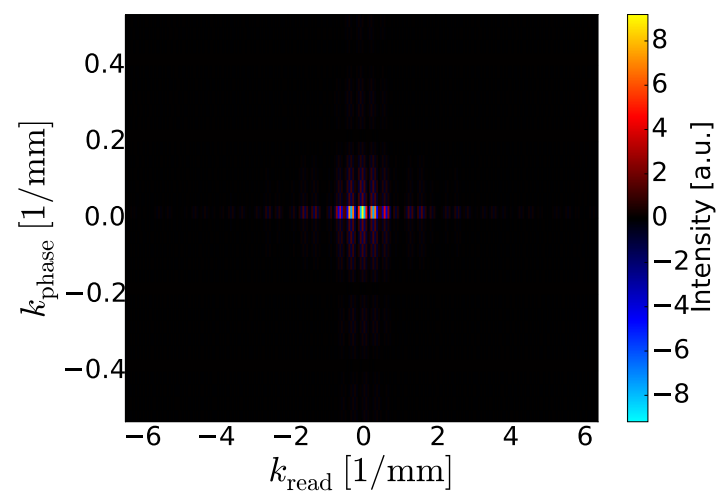

(b)

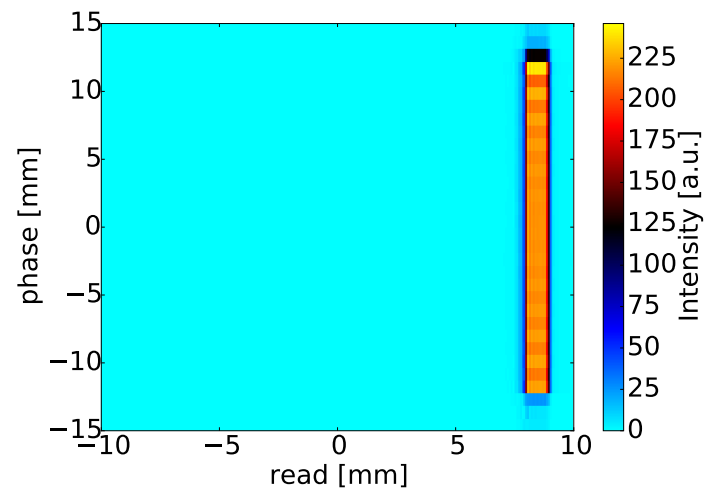

Fig. 4.4: (a) The acquired signal $S(t)$ (shown: real part) is the sum over the magnetization of all isochromats at $t$. A RARE-type sequence was used in the present case. The values $N_{r}, N_{\varphi}$ and $N_{z}$ were all set to 50. (b): MR image of the Couette gap computed by Fourier transformation of the acquired signal from (a).

Due to the nature of the underlying Bloch equations, a parallelization of the simulation program is simple. To this end, the simulation grid is partitioned into equally sized blocks for which the NMR signal is computed individually by each of the processors. After each processor has finished the computation for the assigned reduced grid, the signals from each process at any sample time $t$ are added up. Running the simulation on $N_{\text {proc }}$ processors 
reduces the simulation time by a factor $1 / N_{\text {proc }}$ for the cases considered in this study. As an effect, the NMR signal from a PGSE-RARE sequence with experimental parameters typical for the present study can be computed in the order of only a few minutes on a conventional personal computer for $N=10^{6}$.

\subsection{Soft pulse simulation}

For the study of selective excitation (see section 2.1.9) a second simulation program was written. Due to the non-linearity of the underlying Bloch equations, analytical solutions for the evolution of the magnetization during a soft pulse are usually difficult to obtain (see e.g. Hoult (1979)). Therefore a common approach is to numerically solve the Bloch equations under these conditions (see e.g. Locher (1980), Callaghan (1993)). Apart from the numerical solver, the 'SliceSelection' program offers a graphical user interface, which allows for a convenient and fast manipulation of simulation parameters.

\subsubsection{Underlying Bloch Equations and Numerical Solver}

The Bloch equations (Eq. (2.5)) are solved in the rotating frame under on-resonant conditions. Without loss of generality the direction of the gradient used is always $z$, i.e. $\mathbf{G}=\left(0,0, G_{z}\right)$. In the case where a (shaped) rf-pulse is applied with $x$ as the rotation axis $\left(B_{x}=B_{1}(t)\right)$ under a steady gradient $G_{z}$ the Bloch equations take the following form:

$$
\begin{aligned}
\frac{\mathrm{d} M_{x}}{\mathrm{~d} t} & =\gamma M_{y} G_{z} z \\
\frac{\mathrm{d} M_{y}}{\mathrm{~d} t} & =\gamma\left(M_{z} B_{1}(t)-M_{x} G_{z} z\right) \\
\frac{\mathrm{d} M_{z}}{\mathrm{~d} t} & =-\gamma M_{y} B_{1}(t)
\end{aligned}
$$

For an arbitrary phase offset $\Delta \phi$ of the soft pulse, i.e. $B_{x}=B_{1}(t) \cos \phi$ and $B_{y}=$ $B_{1}(t) \sin \phi$, the equations are slightly more complicated :

$$
\begin{aligned}
\frac{\mathrm{d} M_{x}}{\mathrm{~d} t} & =\gamma\left(M_{y} G_{z} z-M_{z} B_{1}(t) \sin \phi\right) \\
\frac{\mathrm{d} M_{y}}{\mathrm{~d} t} & =\gamma\left(M_{z} B_{1}(t) \cos \phi-M_{x} G_{z} z\right) \\
\frac{\mathrm{d} M_{z}}{\mathrm{~d} t} & =\gamma B_{1}(t)\left(M_{x} \sin \phi-M_{y} \cos \phi\right)
\end{aligned}
$$

The set of Eqs. (4.3) is referred to as the 'no trick' set within context of the simulation program. In order to make the differential equations more symmetric one can consider 
this situation in another reference frame. This reference frame rotates about the $z$-axis at an angular frequency $\gamma G_{z} z$ relative to the rotating frame and accounts for the change in precision frequency due to the applied gradient. In particular, it shall be noted that this new reference frame $\left(x^{\prime}, y^{\prime}, z^{\prime}\right)$ is $z$-dependent. Therefore it is useful to focus on a single value of $z$ while developing the transformed differential equation. If the two reference frames coincide at the start of the pulse when $t=-T$, then the transformed version of Eqs. (4.3) read as follows:

$$
\begin{aligned}
\frac{\mathrm{d} M_{x^{\prime}}}{\mathrm{d} t} & =-\gamma M_{z^{\prime}} B_{y^{\prime}} \\
\frac{\mathrm{d} M_{y^{\prime}}}{\mathrm{d} t} & =\gamma M_{z^{\prime}} B_{x^{\prime}} \\
\frac{\mathrm{d} M_{z^{\prime}}}{\mathrm{d} t} & =\gamma\left(M_{x^{\prime}} B_{y^{\prime}}-M_{y^{\prime}} B_{x^{\prime}}\right)
\end{aligned}
$$

with

$$
\begin{aligned}
& B_{x^{\prime}}=B_{1}(t) \cos \left[\gamma G_{z} z(t+T)+\Delta \phi\right] \\
& B_{y^{\prime}}=B_{1}(t) \sin \left[\gamma G_{z} z(t+T)+\Delta \phi\right]
\end{aligned}
$$

This trick goes back to Bailes and Bryant (1984) and is described in more detail in Callaghan (1993). Please note, that the soft pulse ends at $t=T$. The set of Eqs. (4.4) is called 'BB' within the simulation program.

A fourth order Runge-Kutta algorithm (Press et al. (2007)) is used to find a numerical solution to the coupled differential equations Eq. (4.3) and Eq. (4.4). The stepsize of the algorithm is adjustable by the user. Convergence of the numerical method is assumed if an increase in stepsizes does not significantly alter the obtained solution. Furthermore, it shall be pointed out that the differential equations do not contain terms describing spin relaxation, which is therefore not considered for the simulation.

\subsubsection{Simulation Procedure}

As a first step, $N$ points are equally spaced along the simulation range $\left[-z_{0}, z_{0}\right]$ with $N$ and $z_{0}$ defined by the user. To each point a magnetization vector $\mathbf{m}_{j}=\left(m_{x, j}, m_{y, j}, m_{z, j}\right)$ $(j=1 \ldots N)$ is attached. The initial values for $\mathbf{m}_{j}$ are the same for all points and set by the user. In particular, this means that a constant spin density is assumed along the $z$-range. After the initialization, the evolution of each magnetization vector $\mathbf{m}_{j}$ under the pulse sequence shown in Fig. 4.5 is computed by treating each point as an isochromat. 
The temporal starting point for the simulation is $t=-\left(T+t_{\mathrm{o}}\right)$. Between $-\left(T+t_{\mathrm{o}}\right)$ and $-T$ only the effect of the constant gradient $G_{z}$ has to be taken into account, which causes a rotation of the transverse magnetization component. The soft pulse is active under the period $-T$ and $T$. In this time range the numerical solver described in section 4.2 is used to compute the magnetization vector $\mathbf{m}_{j}(T)$. The underlying differential equation ('BB', 'no trick') is chosen by the user. After the application of the soft pulse, the signal

$$
S(t)=\sum_{j=1}^{N}\left(m_{x, j}(t)+i m_{y, j}(t)\right)
$$

is sampled at a constant rate under the gradient $G_{z}$ until $t=T+2 t_{\mathrm{o}}$. Please note, that it is necessary to transform back $\mathbf{m}_{j}$ from the $z$-dependent $\left(x^{\prime}, y^{\prime}, z^{\prime}\right)$ reference frame into the rotating frame before the signal can be acquired in case the 'BB' set was chosen. At $T+t_{\mathrm{o}}$ the formation of an echo is expected if the initial transverse magnetization is not zero. Furthermore, the output of $m_{\text {total }}=\left(m_{1}, \ldots, m_{N}\right)$ at the echo position is possible.

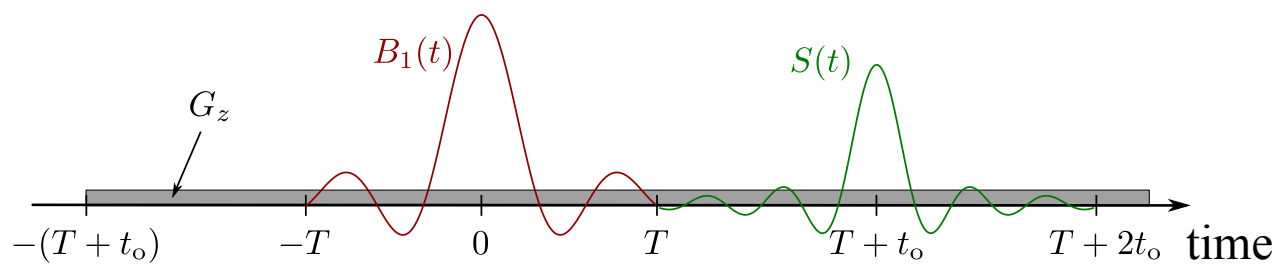

Fig. 4.5: Pulse sequence for the simulation program.

Parallelization of the simulation process is achieved in the same way as for the 'velosim' program. $N$ points along the simulation range are evenly distributed between the processors, which independently solve the Bloch equations. The full signal is then given by adding up the signal from all subprocesses. Convergence is typically reached for a simulation time in the order of minutes on a personal computer for $N=10^{5}$. Furthermore, the 'BB' differential equations were taken as the solver throughout all simulation experiments presented here. It has been found that Runge-Kutta algorithm converges with a smaller stepsize for this equation set, in particular for points further away from the slice centre. This might be related to the symmetry of the underlying differential equations in the ' $\mathrm{BB}$ ' set.

With regards to an implementation of the soft pulse program into the RARE simulation sequence, it can be stated that the computational effort would be enormous at the current stage. Simulating multiple soft pulses for $10^{6}$ grid points, commonly used for the 'velosim' 
program, would take hours to days depending on the hardware available and is thus not feasible. Further optimization of the simulation routines however, might make the combination of both programs possible in the future.

\subsubsection{Comparison to 'imslice’ Experiment}

A pulse sequence similar to the one shown in Fig. 4.5 is used in NMR experiments to determine the power/amplitude needed for the soft pulse $B_{1}(t)$ in order to achieve a certain nutation angle. The main difference is the application of a hard $90^{\circ}$ excitation pulse at $t=-\left(T+t_{o}\right)$, which is necessary to create transverse magnetization. This sequence is known as 'imslice' in the Bruker software 'Topspin'. By Fourier transforming the acquired signal $S(t)$ one obtains a 1D image of the excited slice, which is weighted by the spatially dependent excitation strength of the soft pulse. The following pulse shape is used throughout the thesis for the soft excitation:

$$
B_{1}(t)=A \operatorname{sinc}\left(3 \frac{t}{T}\right) \exp \left(-10\left(\frac{t}{2 T}\right)^{2}\right) \text { for } t \in[-T, T]
$$

with the (normalized) sinc function being defined as

$$
\operatorname{sinc}(x)=\frac{\sin (\pi x)}{\pi x} .
$$

The pulse shape has the shape of a sinc function truncated at $|x|=3$ on each side with a Gaussian envelope. The pulse shall be referred to as 'sinc3G10' and is shown in Fig. 4.6.

In Fig. 4.7 the resulting signal and slice profile for the 'sinc3G10' pulse is shown for both the simulation and the corresponding NMR experiment. Good agreement is found proving the usability of the program. Furthermore, it shall be mentioned that for both the simulation and the experiment, $T$ was set to $1 \mathrm{~ms}$. This value for the soft pulse length has been used for all experiments throughout the thesis (if applicable), if not stated otherwise. 


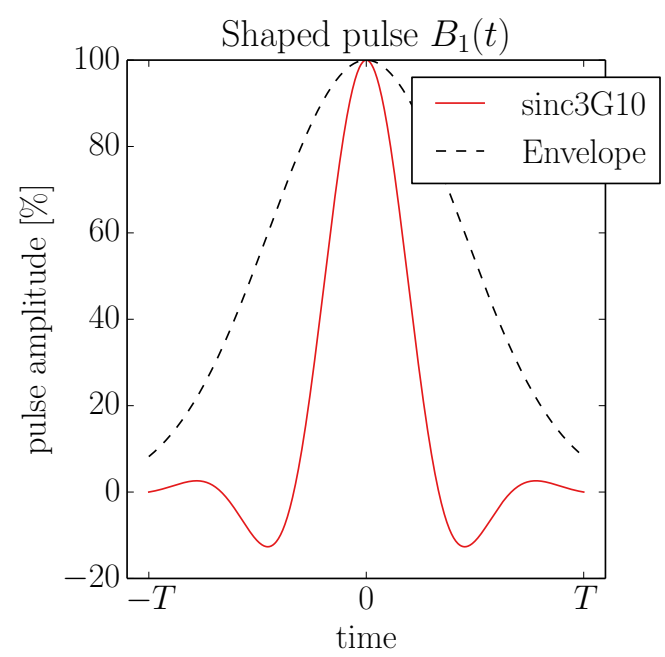

Fig. 4.6: 'sinc3G10' pulse and Gaussian envelope.

(a)

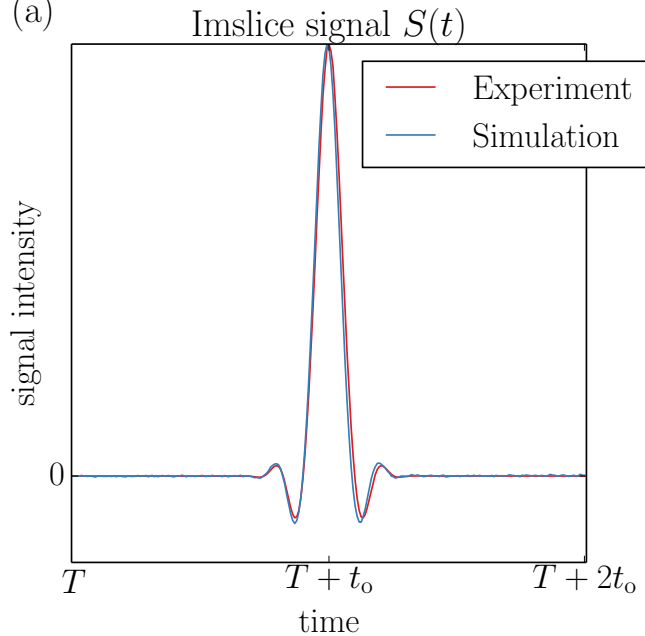

(b) $1 \mathrm{D}$ slice profile $\mathrm{Ft}(S(t))$

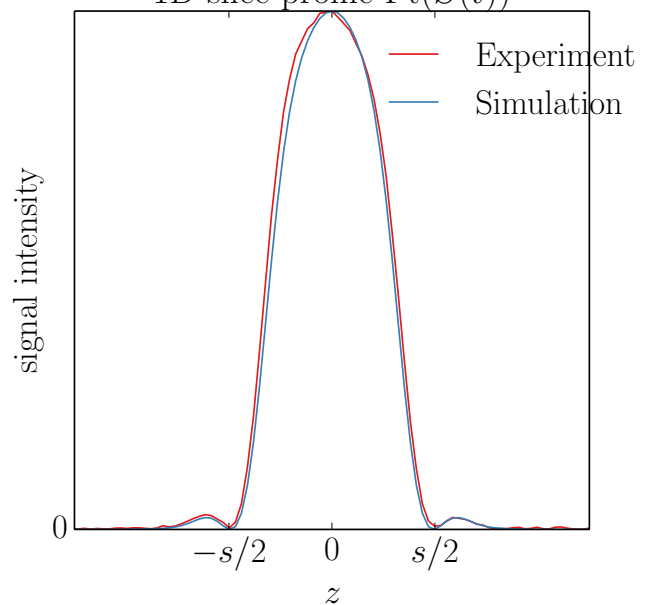

Fig. 4.7: (a) Comparison of simulated signal obtained with the sequence in Fig. 4.5 and the signal obtained from an equivalent 'imslice' experiment with the 'sinc3G10' pulse. Note, that the simulation signal is scaled such that the signal maximum coincides with that of the experiment. (b) 1D intensity profiles for simulation and experiment obtained by Fourier transform of the recorded signal from (a). The thickness of the slice is denoted by $s$. Similarly to (a), the simulation profile was scaled such that the maximum equals the one of the experiment. 


\section{Bibliography}

D. R. Bailes and D. I. Bryant. NMR imaging. Contemporary Physics, 25(5):441-475, 1984. ISSN 0010-7514. doi: 10.1080/00107518408210722. URL http://www.tandfonline. com/doi/abs/10.1080/00107518408210722.

Paul Callaghan. Principles of Nuclear Magnetic Resonance Microscopy. Oxford University Press, 1993. ISBN 9780198539971.

Mattias Edén. Computer simulations in solid-state NMR. i. spin dynamics theory. Concepts in Magnetic Resonance Part A, 17A(1):117-154, January 2003. ISSN 1552-5023. doi: 10.1002/cmr.a.10061. URL http://onlinelibrary.wiley.com/doi/10.1002/ cmr.a.10061/abstract.

D. I Hoult. The solution of the bloch equations in the presence of a varying b1 field-An approach to selective pulse analysis. Journal of Magnetic Resonance (1969), 35(1): 69-86, July 1979. ISSN 0022-2364. doi: 10.1016/0022-2364(79)90078-7. URL http: //www.sciencedirect.com/science/article/pii/0022236479900787.

J.D. Hunter. Matplotlib: A 2D graphics environment. Computing in Science Engineering, 9(3):90-95, May 2007. ISSN 1521-9615. doi: 10.1109/MCSE.2007.55.

P. R. Locher. Computer simulation of selective excitation in n.m.r. imaging. Philosophical Transactions of the Royal Society of London. B, Biological Sciences, 289(1037):537542, June 1980. ISSN 0962-8436, 1471-2970. doi: 10.1098/rstb.1980.0073. URL http: //rstb.royalsocietypublishing.org/content/289/1037/537. PMID: 6106229.

W. H. Press, S. A. Teukolsky, W. T. Vetterling, and B. P. Flannery. Numerical Recipes, The Art of Scientific Computing. Cambridge University Press, 3 edition, 2007.

Tony Stöcker, Kaveh Vahedipour, Daniel Pflugfelder, and N. Jon Shah. High-performance computing MRI simulations. Magnetic Resonance in Medicine, 64(1):186-193, 2010. ISSN 1522-2594. doi: 10.1002/mrm.22406. URL http://onlinelibrary.wiley.com/ doi/10.1002/mrm.22406/abstract.

S. van der Walt, S.C. Colbert, and G. Varoquaux. The NumPy array: A structure for efficient numerical computation. Computing in Science Engineering, 13(2):22-30, March 2011. ISSN 1521-9615. doi: 10.1109/MCSE.2011.37. 
Mikhail Veshtort and Robert G. Griffin. SPINEVOLUTION: a powerful tool for the simulation of solid and liquid state NMR experiments. Journal of Magnetic Resonance, 178(2):248-282, February 2006. ISSN 1090-7807. doi: 10.1016/j.jmr.2005.07.018. URL http://www.sciencedirect.com/science/article/pii/S1090780705002442. 



\section{RARE Velocimetry for Cylindrical Couette Flow}

The main objective of this chapter is a description of how to accurately measure flow in a cylindrical Couette geometry using a PGSE-RARE pulse sequence. The cylindrical Couette cell is a device commonly used in rheological characterization of materials. However, it is usually bulk quantities such as the bulk shear stress that are measured. Therefore, the measurement of local fluid velocities is a way of making the sample characterization more complete and can offer insights into underlying mechanism of the material under study. In particular, the focus shall be on wormlike micellar solutions that exhibit a flow phenomenon called shear banding where the flow separates in two bands of differing fluid viscosity.

As outlined in section 3.2.1.1, the flow in the Taylor-Couette cell is of cylindrical nature with the tangential velocity component being of interest for the flow measurement. For shear banded flow, this component might not only be a function of the radius $r$ but also of the axial position $z$. Thus, the goal is to capture $v_{\varphi}(r, z)$ with the $2 \mathrm{D}$ velocimetry method. In essence two main difficulties have to be mastered. The first is concerning the fact that the imaging process is bound to a Cartesian grid in contrast to the cylindrical symmetry of the flow. Consequently, the sample region that is coherently excited during the pulse sequence has to be chosen carefully in order to get a quasi equivalence of a measured Cartesian flow component and $v_{\varphi}(r, z)$. Furthermore, attention has to be paid to the curved motion of the fluid during the $k$-space acquisition. The second difficulty lies in the small extent of the fluid gap for the Couette cells used in this study. In order to get a sufficient number of data points in the direction of the velocity gradient, a high resolution is necessary, which in turn leads to a small voxel volume and a potentially low SNR in each data point. To this end, alterations to the PGSE-RARE sequence are presented that allow for high resolution measurements and more accurate description of the flow dynamics. 
The chapter starts with a brief description of the experimental setup. Afterwards theoretical aspects and the implementation of the PGSE-RARE pulse sequence are discussed in detail. In particular, the simulation programs described in chapter 4 are used to further the understanding of measurement artefacts. As a result, recommendations regarding the choice of measurement parameters are given. Subsequently, these recommendations are supported by experimental results. Finally, the potential of a high resolution version of the PGSE-RARE sequence is shown.

\subsection{Design of Rheo-NMR Hardware}

A schematic of the experimental setup is shown in Fig. 5.1. The top of the Couette cell is coupled to a drive shaft that is in turn connected to a stepper motor sitting on top of the magnet shell. The rotational frequency of the motor can be controlled via commands within the pulse sequence and thus allows for a precise setting of the applied shear rate $\dot{\gamma}_{a}$ at any time during the experiment. This way of imposing the motion has been used for previous work in our lab and is described in detail in Callaghan (2007) for example. It essentially corresponds to the shear-controlled mode offered by commercial rheometers. Measuring the torque/stress exerted by the fluid onto the drive-shaft is not possible in the present setup. Stress measurements for RheoNMR experiments are difficult due to the presence of high magnetic fields, although not impossible as shown by Grabowski and Schmidt (1994). The coil used for all NMR experiments was a $25 \mathrm{~mm}$ standard Bruker birdcage rf coil as provided by the MICRO2.5 micro imaging system.

Two different custom build cylindrical Couette cells have been used for the studies presented in this work. The first cell (A) had a total diameter of $25 \mathrm{~mm}$ and both cylinders were made of polyetheretherketone (PEEK). PEEK allows for an easy manipulation of surface properties and is therefore useful for studying slip effects, for example. Radii of $10.25 \mathrm{~mm}$ and $11.75 \mathrm{~mm}$ for the rotor and the inner wall of the stator respectively resulted in a gap width of $1.5 \mathrm{~mm}$. In order to create a mechanical feature in the $z$-direction, Teflon tape was wrapped around the inner cylinder decreasing the effective gap size locally (Fig. 5.4). The $z$-axis of the cell is aligned with the direction of the magnetic field.

The design of the second cell (B), which was developed in our research group by a fellow $\mathrm{PhD}$ student (Tim Brox), was aiming for a more robust control of thermal conditions of the sample fluid as compared to cell A. Even slight temperature changes in the order of $1{ }^{\circ} \mathrm{C}$ can alter the behaviour of complex fluids considerably (see e.g. Berret et al. (1997)). Therefore active thermal control in the magnet via an air stream provided by the Bruker kit is advantageous. As cell A fills out the whole space of the $25 \mathrm{~mm}$ rf coil sufficient air flow 


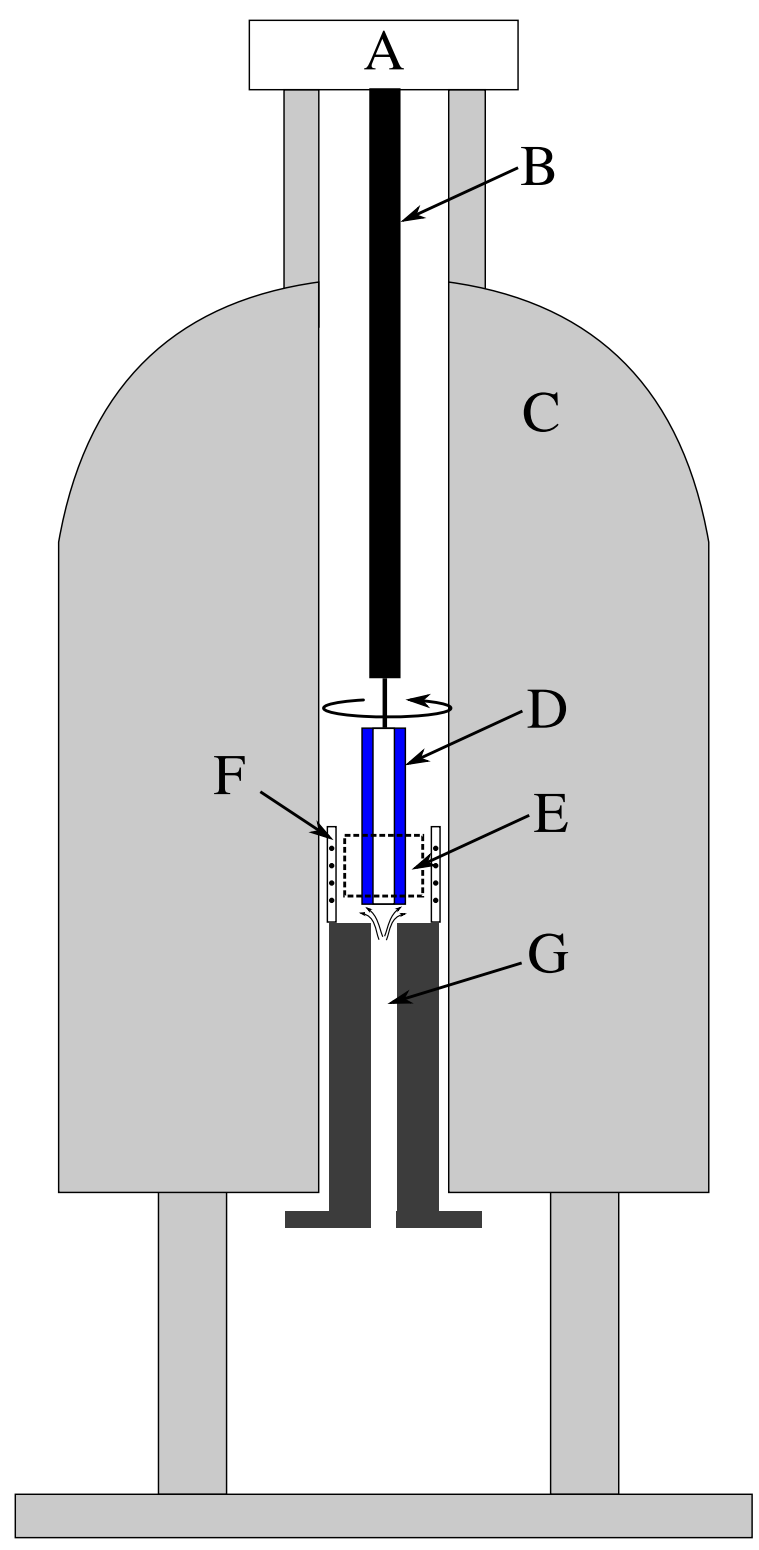

Fig. 5.1: Schematic of the experimental setup. A. Strain controlled stepper motor. B. Drive shaft connecting motor and Couette cell. C. Shell of superconducting magnet. D. Cylindrical Couette cell. E. Imaging region. F. Rf coil. G. Access for air flow. 
around the geometry cannot be generated. Hence, thermal energy emitted by the gradient and rf coils can heat up the sample during the course of a potentially long experiment. A schematic of Couette cell B is depicted in Fig. 5.2. The total diameter of the cell has been reduced to $20 \mathrm{~mm}$ providing space for air to flow along the outer wall while inserted in the magnet. Spacers at the bottom and in the middle of the cell were designed to keep to the structure centred in the rf coil and to allow air to pass through. The inner radius was chosen to be $8 \mathrm{~mm}$ and the gap width $d=1 \mathrm{~mm}$. The decrease in gap width $d$ as compared to cell A was necessary to ensure a small variance in stress over the gap. As the material for the outer cylinder, precision bore glass tubing (GPE Scientific Limited) was preferred over PEEK, as it allowed for optical access of the fluid while outside of the magnet. This is particularly useful for the detection of air bubbles that are a common problem when dealing with complex fluids. As it can be seen in Fig. 5.2, the cell extends well beyond the coil region at the top. The coil region itself is approximately given by the position of the spacers. This allows for the minimization of end effects within the coil region that are induced by the air-fluid interface at the top of the cell. It shall be mentioned that $25 \mathrm{~mm}$ coil was the largest available working with both our probe and the Couette cells. Larger coils and therefore more space for the shearing geometry are available for ultra-wide bore or certain low-field magnets. For both geometries the inner cylinder was hollow to allow for the insertion of a marker fluid undergoing rigid body rotation. Marker fluids are useful for validating the accuracy of the velocimetry measurement due to the known flow profile and can be used to accurately determine the position of the gap in the recorded flow map, as shall be discussed in section 5.2.5.

\subsection{Theory and Implementation}

\subsubsection{The PGSE-RARE pulse sequence}

The pulse sequence allowing for the acquisition of quantitative $2 \mathrm{D}$ velocity maps is a combination of a pulsed-gradient spin-echo (PGSE) and rapid acquisition with relaxation enhancement (RARE) sequence. It is based on former work in our lab, in particular by Galvosas and Callaghan (2006), Feindel and Callaghan (2010) and Brown and Callaghan (2011). All experiments were performed on a Bruker Avance 400 spectrometer equipped with a Micro-2.5 tri-axial gradient system with a maximum magnetic field gradient of $1.45 \mathrm{~T} / \mathrm{m}$. A birdcage $\mathrm{rf}$ coil with an inner diameter of $25 \mathrm{~mm}$ was used for the excitation and the detection of the ${ }^{1} \mathrm{H}$ signal. A schematic of the pulse sequence is depicted in Fig. 5.3. 
(a)

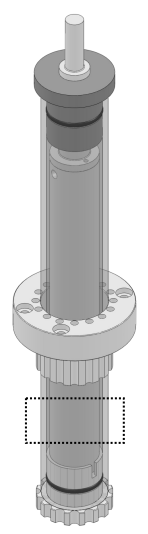

(b)

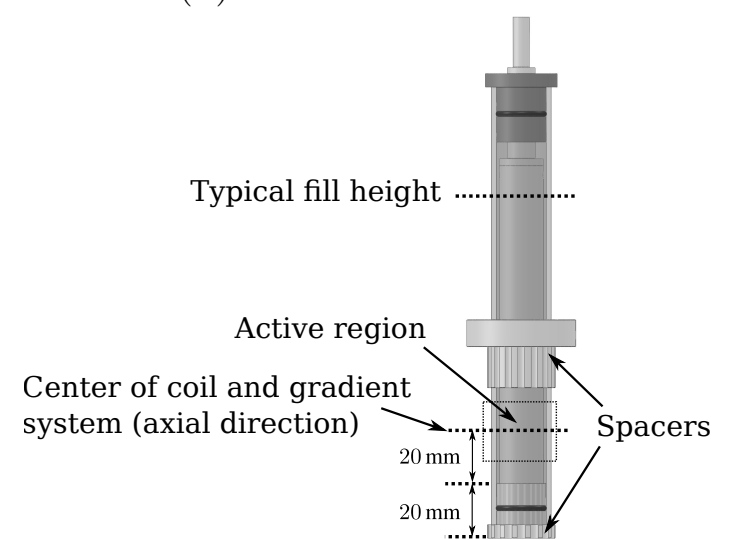

Fig. 5.2: (a) Sketch of the Taylor-Couette cell B used for the NMR experiments. The active region is indicated by the dashed rectangle. (b) Side view of the same cell. The upper dashed line corresponds to a typical fill height for the fluids under study. Also, measures at the bottom of the Couette cell are given and the centre of the coil and gradient system in the axial direction is indicated.
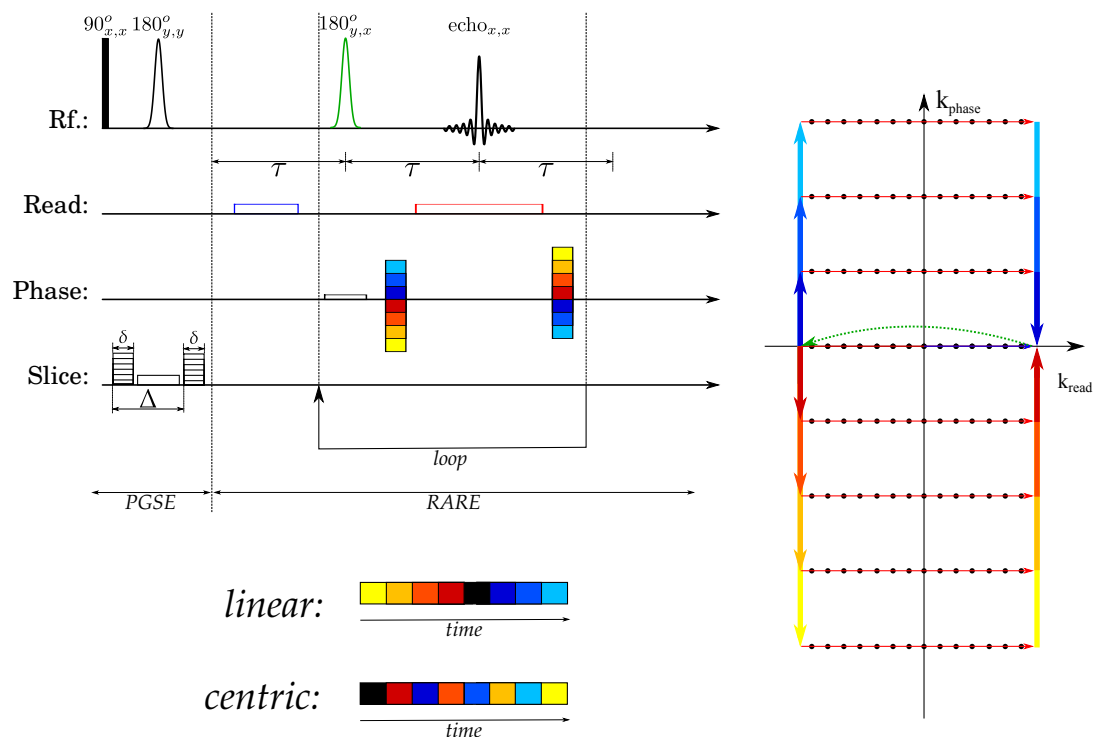

Fig. 5.3: PGSE-RARE sequence used to record $2 \mathrm{D}$ velocity maps. Also shown are two different $k$-space trajectories, namely centric and linear. Black means no (phase) gradient. 
The underlying principles of the PGSE part of the pulse sequence have been described in section 2.2.2. A pair of pulsed gradients with a refocusing pulse in between creates a velocity contrast that can later be mapped on an MRI image. The refocusing pulse is chosen to be a soft selective pulse, which excites a slice normal to the $y$-direction as shown in Fig. 5.4b. This slice shall be called 'PGSE slice'. The velocity encoding is also applied in the $y$-direction, as the objective is to measure the flow into and out of the (PGSE) slice, given by the $y$-component of the underlying flow field, denoted by $v_{y}$. The encoding time $\delta$ and the observation time $\Delta$ were chosen to be equivalent for all experiments with values of $1 \mathrm{~ms}$ and $20 \mathrm{~ms}$, respectively, similar to what has been used by Feindel and Callaghan (2010) in a previous PGSE-RARE study. Furthermore, it was made sure that displacements under the $\Delta$ period were small $(0.5 \mathrm{~mm}$ maximal $)$ so that the velocity encoding is not significantly affected by the centripetal acceleration of the sheared fluid.

Due to the curved stream-lines in the Couette geometry care has to be taken in regards to the choice of the (PGSE) slice thickness. These considerations are presented in section 5.2.2.1. The second part of the sequence (RARE), allows the velocity contrast to be mapped to a 2D MRI image. The normal of the selected slice is shifted to the phase direction, as indicated in Fig. 5.4a. In doing so, fold-over artefacts originating from coherent signal generated outside the field of view are removed. However, this way of imposing the slice selection comes at the cost of an intensity scaling in the phase direction and a reduced quality of the flow measurement at the edges of the FOV as shown later. This issue regarding the choice of the 'RARE slice' is discussed in section 5.2.2.2. The two slices define the active volume for the experiment as illustrated in Fig. 5.4. In order to maintain quadrature during the echo train, i.e. conserving the phase information stored in the magnetization, the phase of the RARE soft pulse is cycled according to Fig. 5.3. This phase cycle and alternatives are discussed in section 5.2.4. Of particular interest is also the $k$-space trajectory. In section 5.2.3 it shall be shown how the choice of a particular $k$-space scheme can influence the velocity measurement due to the motion of the fluid under the imaging part of the pulse sequence.

The final 2D MRI image acquired with the PGSE-RARE sequence contains both magnitude and phase information. From the signal magnitude structural information on the system can be obtained. This is of importance for the precise localization of the Couette gap position as will be discussed in section 5.2.5. The velocity information for each voxel is stored in the phase $\varphi$ of the signal. Ideally it is related to the velocity $v_{y}$ via $\varphi=\gamma g \delta \Delta v_{y}$, where $g$ is the strength of the flow gradient. As discussed in section 2.2, imperfections in the applied gradients and a non-uniform magnet field over the sample can cause addi- 
(a)

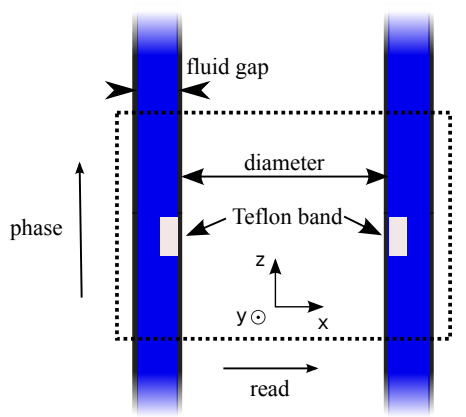

(b)

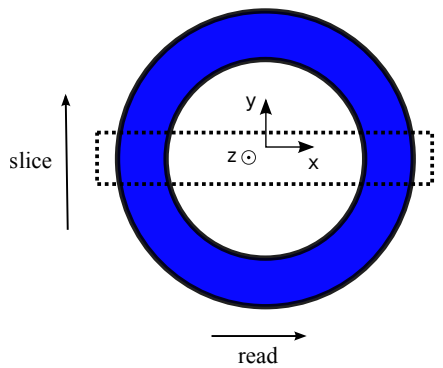

Fig. 5.4: Schematics of vertical (a) and horizontal (b) cuts through the cylindrical Couette geometry. The active volume/region given by the slice selection of the NMR pulse sequence is indicated by the dashed rectangle. The Teflon band shown in (a) is used for cell A to create a (flow) feature in the phase direction.

tional phase accumulation which cannot be easily separated from phase shifts due to the velocity encoding. In order to eliminate these additional contributions two phase images are acquired with different flow gradients $g_{1}=0$ and $g_{2} \neq 0$. Subsequently only the phase difference between those two images is used for the velocity calculation.

A typical result for a 2D flow map acquired with the pulse sequence is depicted in Fig. 5.5b. The corresponding magnitude information is shown in Fig. 5.5a. In both maps, the two gaps, which are denoted 'gap1' and 'gap2', are found on the left and the right side, respectively. The marker fluid appears between the two gaps. The flow directions for 'gap1' and 'gap2' are opposite to each other in agreement with the circular flow in the Couette geometry. From the flow maps, 1D velocity profiles can be extracted for each position along the phase direction as shown in Fig. 5.6 in form of a waterfall plot.

\subsubsection{Slice Selection}

\subsubsection{PGSE}

Stream lines in the Couette geometry are of cylindrical symmetry, as the applied shear imposes a circular motion on the fluid contained in the cell (see section 3.2.1). The imaging and slice selection process works on a Cartesian grid however, as constant gradients are applied in the desired directions in order to spatially resolve the studied object or to excite a certain rectangular region of the sample (section 2.1.8). Capturing the flow of the cylindrical Couette cell in a meaningful way is therefore a challenge for magnetic resonance velocimetry in general and for the PGSE-RARE sequence in particular. The main flow 

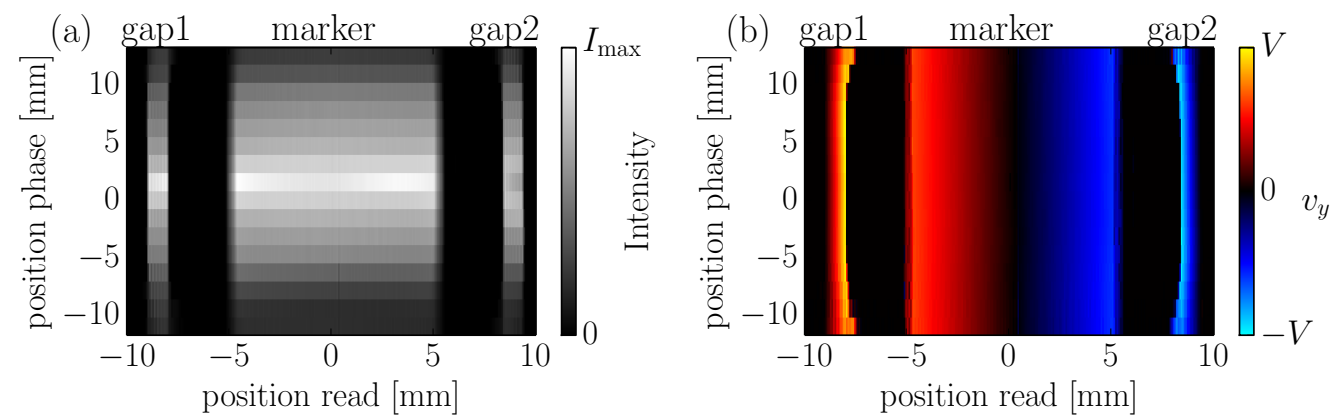

Fig. 5.5: Typical outcome of the 2D PGSE-RARE experiment for cell B: Magnitude information (a) and flow map (b) resulting from phase contrast. Read and phase correspond to $x$ and $z$ respectively as defined in Fig. 5.4. The field of view is $20 \mathrm{~mm}$ in read and $25 \mathrm{~mm}$ in the phase direction. Deviation in the MRI image and the flow map at the top and bottom edge as well as an intensity scaling in the phase direction in (a) are due to the slice selection in the RARE part of the sequence.

component to be determined is the tangential velocity as a function of radial position, namely $v_{\varphi}(r)$ (see Eq. (3.8)). In contrast, the quantity measured by the NMR sequence is $v_{y}(x, z)$, as described in section 5.2.1. Ideally an infinitely thin slice in the flow direction centred with the Couette geometry would be necessary to get an equivalence of $v_{\varphi}(r)$ and $v_{y}(x)$. In experimental conditions such a slice is not possible as the signal-to-noise ratio for each imaging voxel is proportional to its size. Reducing the slice thickness and with it the voxel volume, will result in a decreased signal intensity for the given voxel and therefore affect the quality of the measurement. Hence, a compromise has to be found between the SNR and the accuracy of the velocity measurement.

The first approach in understanding the influence of the slice thickness is made by analysing the flow of a model fluid which exhibits a linear velocity profile over the gap, as for example expected for a Newtonian fluid such as water in the case of a small geometrical ratio $\Lambda$ (Eq. (3.11)). The dimensions of the confining Couette geometry are chosen to be equal to those of cell $\mathrm{B}$, where the velocity profile is approximately linear in the Newtonian case, as shown in Fig. 3.5. In Fig. 5.7a the tangential velocity field for the model fluid at an applied shear rate of $10 \mathrm{~s}^{-1}$ and a slice thickness of $4 \mathrm{~mm}$ is shown. Please note that the view is restricted to one gap in the active volume region. Dotted vertical lines show the voxel boundaries for a spatial resolution in the read direction typically achieved with the PGSE-RARE sequence (about $80 \mu \mathrm{m}$ ). Due to the curvature of the geometry, a strong variation of velocities is observed in the slice direction for all voxels. This effect is not desired, as the measured velocity at each voxel position is an average over the 


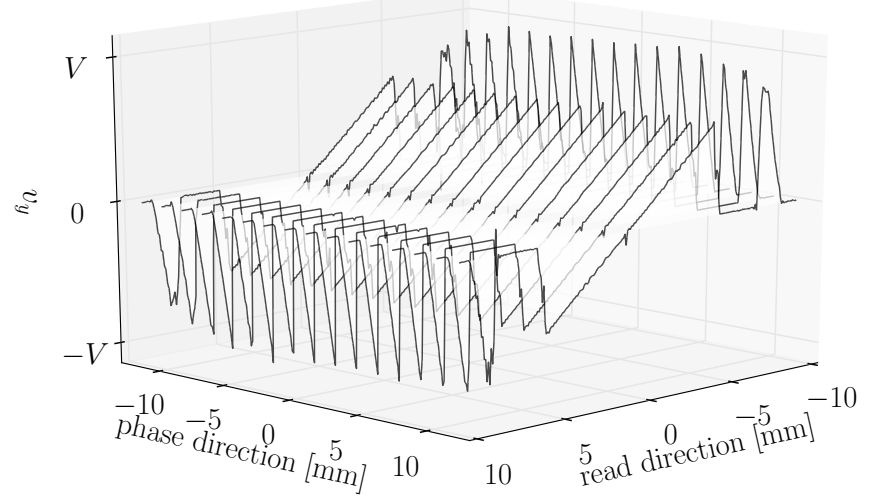

Fig. 5.6: The flow map in Fig. 5.5b as a waterfall plot showing individual $1 \mathrm{D}$ flow profiles along the phase direction.

whole voxel volume. Ideally it should be an average over the extent in the $x$-direction, but not contain a signature of the slice depth in $y$. To analyse this depth effect more quantitatively the average velocity in each voxel volume has been computed numerically in Fig. 5.7b for a range of applied shear rates. The voxel velocities are clearly below the expected values given by the assumed linear flow profile, which is indicated by the dashed lines. Also, additional voxels appear below the inner wall position at $8 \mathrm{~mm}$, which give a wrong impression of the gap extent and the gradient of the velocity profile. Furthermore, a certain amount of slip appears to be present, due to the underestimated fluid velocity at the rotor.

Reducing the slice thickness to $1 \mathrm{~mm}$ (Fig. 5.7c,d) greatly decreases the deviations observed in the $4 \mathrm{~mm}$ case. The average voxel velocities match very well with the actual tangential velocities at each point along the gap. Also no additional velocity voxels are recorded compared to the $4 \mathrm{~mm}$ case and no apparent slip is present. The extraction of shear rates or local velocity gradients is of great interest for the analysis and characterization of the gap profiles. In order to understand the influence of the slice thickness on the apparent velocity gradient the profiles in Fig. 5.7b,d have been fitted with a linear function (solid line). The resulting velocity gradients $\dot{\gamma}_{f i t}$ are shown in the insets of each of those figures. In the $1 \mathrm{~mm}$ case the gradients match almost perfectly with the applied shear rates. For the $4 \mathrm{~mm}$ case slight deviations are observed, which are however relatively small. Therefore a slice thickness of $4 \mathrm{~mm}$ might be acceptable if a linear flow profile over the gap is expected. Increasing the slice thickness could be a means of improving the SNR of the flow map for example. 

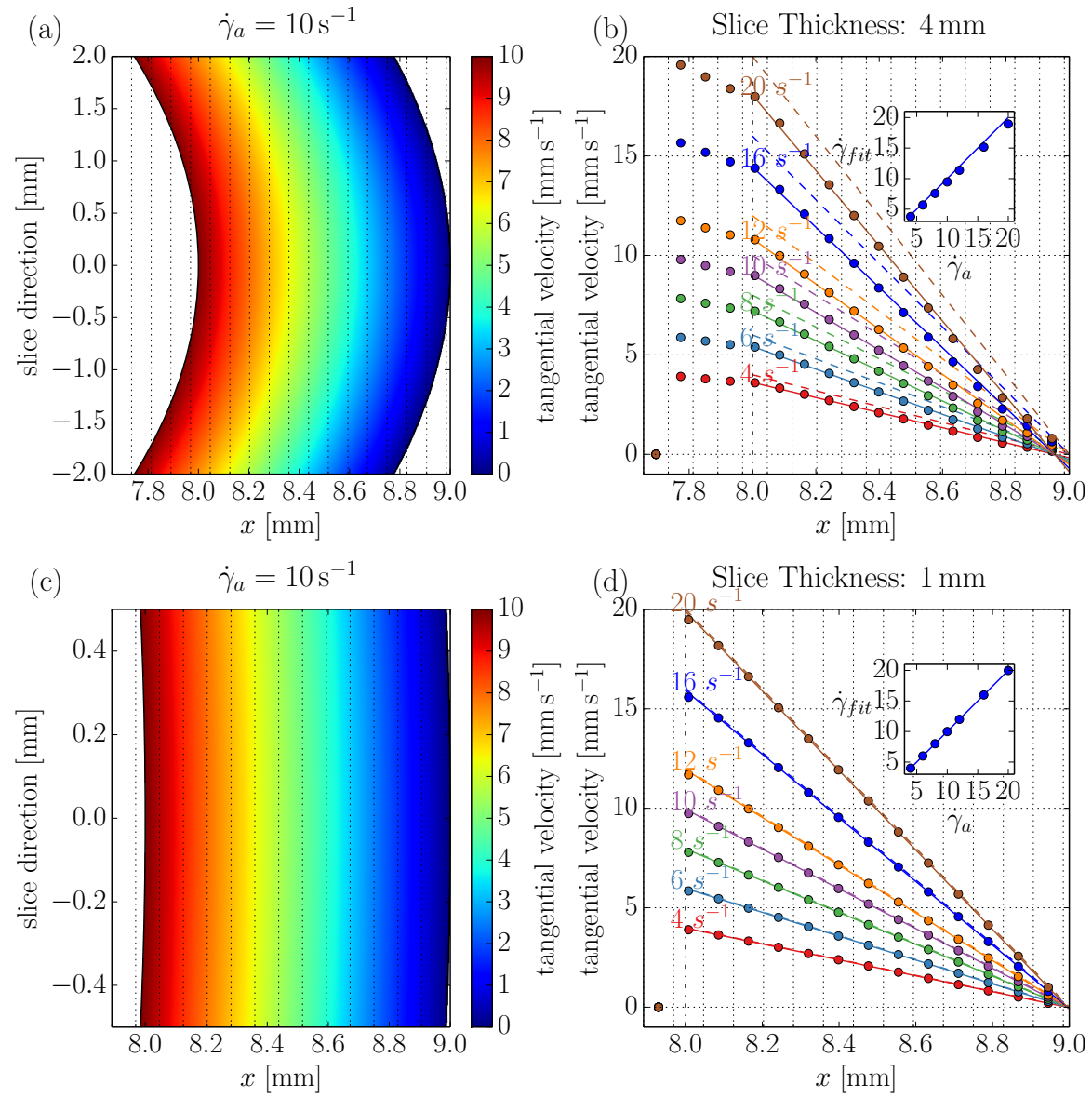

Fig. 5.7: (a) and (c) show flow maps of a model fluid exhibiting a linear profile over the gap at an imposed shear rate $\dot{\gamma}_{a}=10 \mathrm{~s}^{-1}$ confined to a cylindrical Couette geometry with equivalent dimensions as the recently developed NMR cell. The view is restricted to the active volume for slice thicknesses of $4 \mathrm{~mm}$ (a) and $1 \mathrm{~mm}$ (c). In (b) and (d) the average velocity in each voxel (dots) is shown for a range of applied shear rates. Vertical dotted lines indicate the voxel boundaries in each plot and solid lines are linear fits taking only points within the gap range $(8 \mathrm{~mm}-9 \mathrm{~mm})$ into account. The dashed lines indicate the assumed velocity profile. Insets compare the applied shear rate (solid line) with the slope obtained from the linear fit (points). Furthermore, additional points below the inner radius of $8 \mathrm{~mm}$ in the thicker slice case give a wrong impression of the actual gradient in the flow field.

Taking a shear banding fluid on the other hand, the implications of this depth effect are more severe. In Fig. 5.8 the Newtonian-like model fluid has been replaced by a fluid obeying the simple lever rule Eq. (3.14). The values chosen for $\dot{\gamma}_{1}=2.5 \mathrm{~s}^{-1}$ and $\dot{\gamma}_{2}=35 \mathrm{~s}^{-1}$ are typical for the real shear banding fluid presented later. The resulting $1 \mathrm{D}$ velocity 

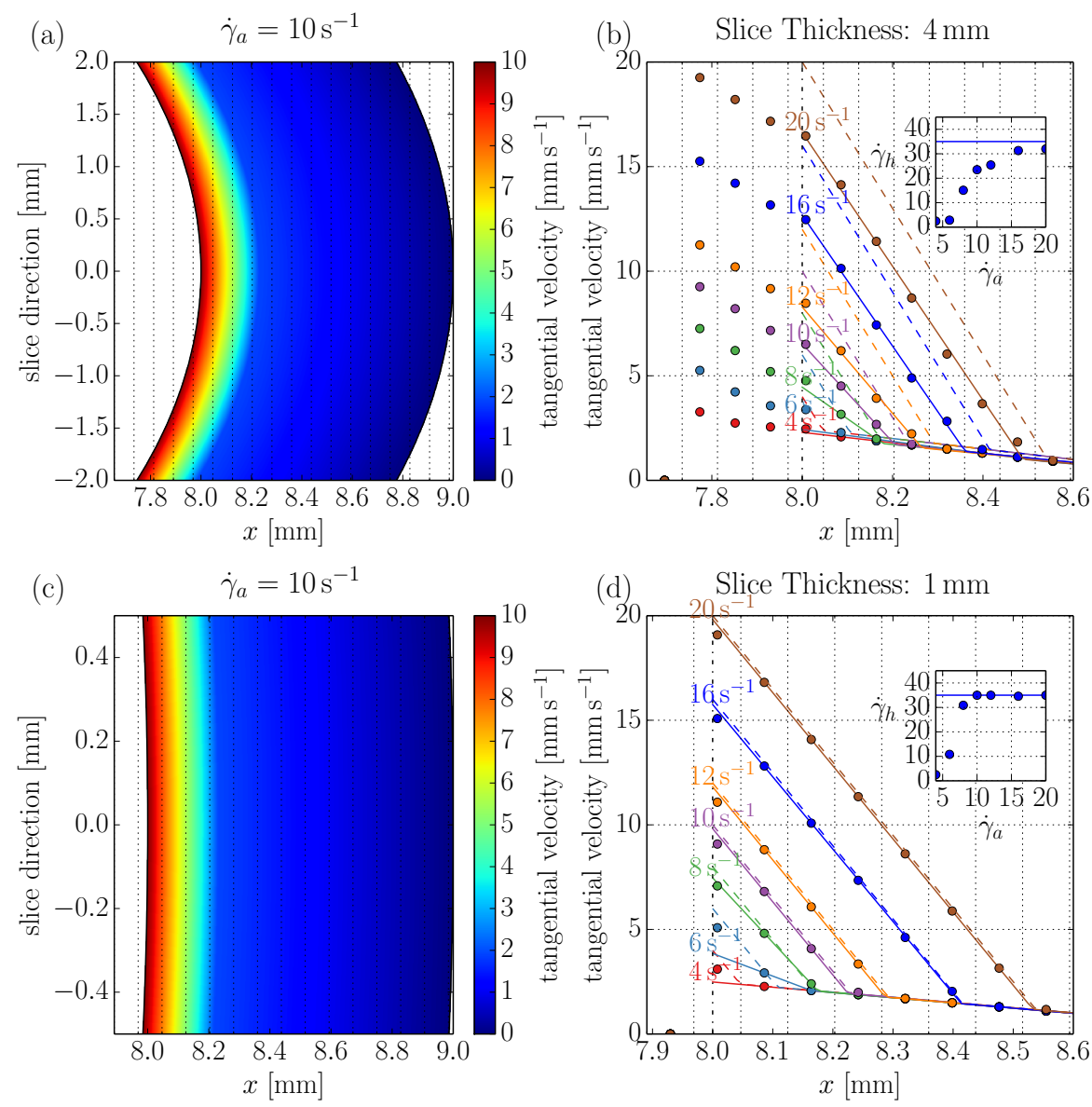

Fig. 5.8: Same as Fig. 5.7, however with a model fluid obeying the simple lever rule Eq. (3.14) with $\dot{\gamma}_{l}=2.5 \mathrm{~s}^{-1}$ and $\dot{\gamma}_{h}=35 \mathrm{~s}^{-1}$. Also, the profiles in (b) and (d) are fitted with a piece-wise linear function assuming two bands instead of simple linear function. The insets show the shear rate $\dot{\gamma}_{h}$ in the high shear rate band. Note that the $1 \mathrm{~d}$ profiles have been truncated at $8.6 \mathrm{~mm}$ to have an enlarged view on the high shear rate region. 
profiles have been fitted with a piece-wise linear function assuming two different gradients in Fig. 5.8b,d. First of all, it is obvious that for both slice thicknesses a high shear rate band smaller than twice the pixel width cannot be accurately measured with the chosen resolution. It shall be noted in this respect that the voxel closest to the inner wall at $8 \mathrm{~mm}$ is not fully situated in the gap range and therefore is not taken into account for the fitting procedure. A way of significantly increasing the gap resolution is presented in section 5.3.3. Starting with applied shear rates of about $8 \mathrm{~s}^{-1}$ the velocity profile is accurately captured in the $1 \mathrm{~mm}$ case as can be seen in Fig. 5.8d. The results for the velocity gradient $\dot{\gamma}_{h}$ in the high shear rate band are depicted in the inset. Indeed a very good agreement of the expected and the fit value is found for $\dot{\gamma}_{a} \geq 10 \mathrm{~s}^{-1}$. In the $4 \mathrm{~mm}$ case the slope of the high shear rate is gradually converging towards the assumed value for increasing applied shear rate (Fig. 5.8b). But even for $\dot{\gamma}_{a}=20 \mathrm{~s}^{-1}$ the fit value deviates by about $10 \%$ as compared to the assumption. This artefact could be mistaken for a flow feature of the complex fluid under study in an experimental setup. Also the amount of apparent slip is more severe as compared to the first case of the Newtonian-like fluid. The extraction of shear rates and volume fractions of the high shear rate band may have been affected in Feindel and Callaghan (2010) and Brown and Callaghan (2011) by choosing $4 \mathrm{~mm}$ thick slices. Additionally, slice thicknesses of $2 \mathrm{~mm}$ and $3 \mathrm{~mm}$ have been analyzed in the same manner, showing similar albeit smaller deviations as in the $4 \mathrm{~mm}$ case. Therefore it shall be concluded that for the present study, a slice thickness of $1 \mathrm{~mm}$ is regarded as a reliable value for an accurate measurement of the radial velocity profile while maintaining a sufficient voxel volume. Thus, the slice thickness for the PGSE part of the velocimetry sequence is set to $1 \mathrm{~mm}$ for all experiments, if not stated otherwise. More generally, a $1 \mathrm{~mm}$ slice corresponds to a slice thickness to inner radius ratio $\Gamma_{\text {slice }}$ of 0.125 for the geometry dimensions assumed here. With respect to an arbitrary cylindrical Couette cell, this value for $\Gamma_{\text {slice }}$ is recommended for a PGSE-RARE experiment.

In general, the optimal slice thickness depends on various factors, such as the dimensions of the flow geometry, the expected flow profile and the available SNR. Thus, careful considerations regarding the slice thickness are always necessary to ensure a meaningful velocity measurement. Furthermore, it shall be pointed out, that this slice effect applies to any velocimetry technique (NMR or not) that relies on the selection of a rectangular slice with a certain thickness in similarity to the PGSE-RARE method. For example, in the case of ultrasonic speckle velocimetry, described in section 6.2 .2 , the width of the active region is given by the incident angle of the ultra sound pulse. Hence, a careful choice of incident angle is necessary in this case. 
Finally, let us come back to the fact that it is actually $v_{y}$ and not $v_{\varphi}$ which is measured with the NMR technique. The local velocity in the $y$ direction is related to the tangential velocity by $v_{y}(x, y)=v_{\varphi}(r) \cos \varphi$ with $\varphi=\arctan (y / x)$ and $r=x^{2}+y^{2}$. However, the projection factor $\cos \varphi$ is very close to 1 over the whole slice area for the slice thicknesses and Couette cells discussed here. Expected deviation caused by the projection are about $2 \%$ for the $4 \mathrm{~mm}$ case and less than $0.1 \%$ for the $1 \mathrm{~mm}$ case. Therefore, a distinction between $v_{y}$ and the tangential velocity $v_{\varphi}$ is regarded artificial for the chosen slice thicknesses.

\subsubsection{RARE}

Applying the slice selection in the phase direction during the RARE part of the velocimetry sequence (Fig. 5.3) is a means of avoiding fold-over artefacts in the phase direction. As a side effect of this procedure however, a scaling of the image intensity in the phase direction is observed in Fig. 5.5a. Furthermore, the velocity measurement is found to be spurious at the edges of the field of view (Fig. 5.5b). The origin of these effects shall be discussed with the help of results obtained from the soft pulse simulation program (section 4.2).
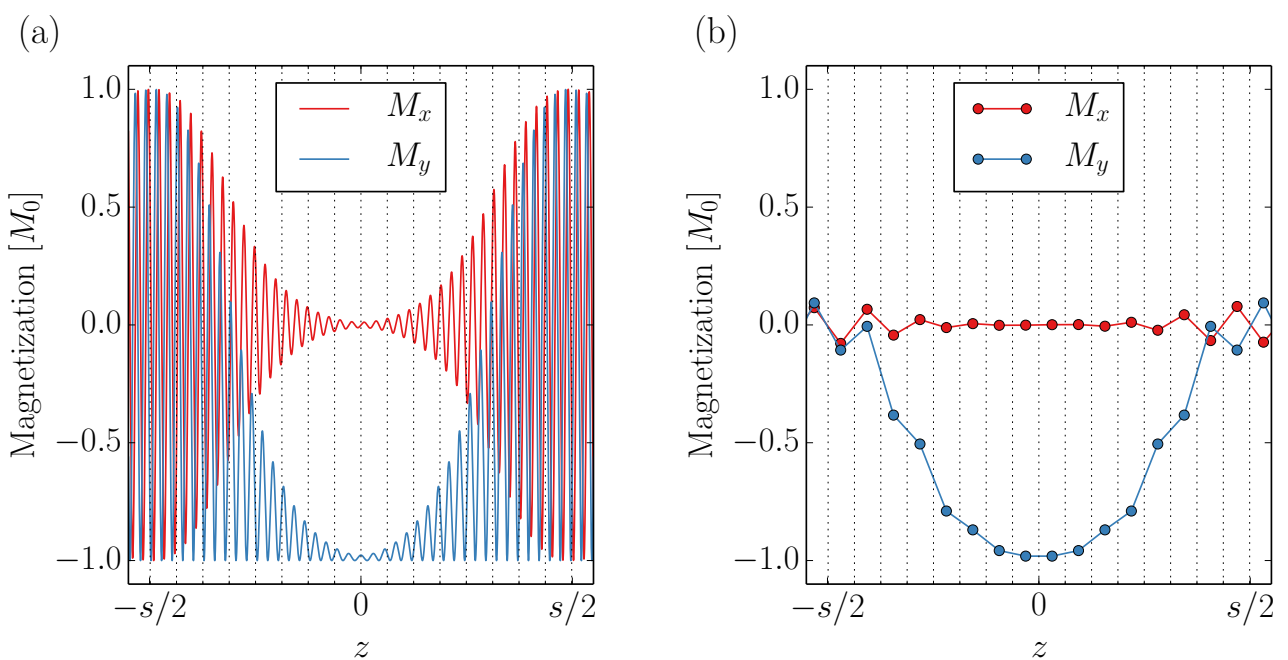

Fig. 5.9: The transverse magnetization at the echo time obtained from the soft pulse simulation for the 'sinc3G10' pulse (a). Averaging over the ranges indicated by the dotted vertical grid lines removes the oscillations observed for $M_{x}$ and $M_{y}$ (b). Please note that $z$ refers to the internal coordinate of the simulation program and not to the $z$-direction of the experimental setup shown in Fig. 5.4. $s$ refers to the thickness of the slice.

The intensity scaling in the magnitude map can be seen in agreement with the $1 \mathrm{D}$ magnitude profile obtained from the 'imslice' experiment and simulation (Fig. 4.7). The 
highest signal intensity is observed in the centre of the slice with a Gaussian-like decay to each side. In order to understand this phenomenon better one can look at the profile of the transverse magnetization at the echo time $\left(T+t_{o}\right)$ for the soft pulse simulation sequence, which is depicted in Fig. 4.5. For the simulation, an initial magnetization $\left(M_{x, 0}, M_{y, 0}, M_{z, 0}\right)=\left(0, M_{0}, 0\right)$ is chosen, along with $x$ as the phase for the $180^{\circ}$ pulse. The resulting transverse magnetization profile at the echo point is shown in Fig. 5.9a. First of all, it is obvious that only in the centre of the slice is a complete inversion of $M_{y}$ found, as would be expected for a perfect refocusing pulse. Moving further from the slice centre, an increasing amount of magnetization is deposited into the $x$-component of the magnetization. Additionally the profile of $M_{x}$ is modulated by an oscillation of constant frequency. $M_{y}(z)$ is oscillating with the same frequency, however between a value of $-M_{0}$ and a seemingly Gaussian shaped envelope. It has been found that the frequency scales inversely with the offset time $t_{o}$ and thus with the degree of dephasing imposed on the transverse magnetization before the soft pulse is applied. With regards to the velocimetry experiment these oscillations are not a main complication, as the resolution in the direction of the slice is expected to be coarser than the wavelength of the undulations. If one averages the magnetization profile over a typical resolution range, which is indicated by the dotted grid lines in Fig. 5.9a, one obtains the profile shown in Fig. 5.9b. There, $M_{x}$ is effectively zero over the whole slice, as expected for the choice of initial magnetization and phase of the refocusing pulse. The profile for $M_{y}$ on the other hand resembles the Gaussian shaped excitation profile observed in the 1D slice image of Fig. 4.7b. Thus, one can state the following. An ideal $180^{\circ}$ rotation is only observed in the centre of the slice. The further one moves away from the centre, the more the average rotation angle deviates from $180^{\circ}$. This explains the Gaussian shaped scaling of the image intensity in Fig. 5.5a. At the edges of the slice, magnetization is not coherently refocused at all. Hence, one obtains slightly spurious velocity measurements on the edge of the field of view as observed in Fig. 5.5b. Furthermore, the oscillations in the profiles of the transverse magnetization are not a major concern, as this (additional) phase pattern is averaged out over the range of typical voxel.

\subsubsection{General Remarks}

The scheme of selecting slices in different directions for the PGSE and RARE part has been applied before by Brown and Callaghan (2011) for a similar PGSE-RARE sequence. As an alternative approach to this double slice selection scheme, the field of view in the phase direction could be extended to cover the whole range from where coherent NMR 
signal is received in order to avoid fold-over artefacts. This range is approximately equal to the length of the coil, which is about $40 \mathrm{~mm}$ in the present case. However, this approach, which has been applied by Feindel and Callaghan (2010), can come at the cost of a reduced resolution in the phase direction due to an increased FOV for otherwise equal imaging parameters. Increasing the number of points in the phase direction on the other hand would prolong the CPMG train, which in turn is not desirable due to relaxation effects. Therefore, the double slice selection scheme has been preferred over the single slice approach even though an intensity scaling as described in the previous section is observed. The thickness of the RARE slice has always been set equal to the FOV in the phase direction for the experiments presented herein.

Furthermore, it shall be mentioned, that in the discussion on the PGSE slice thickness (section 5.2.2.1) the slice was assumed to be perfectly rectangular. However, as shown in the previous section, the central region of the slice has a stronger contribution to the NMR signal than parts which are further away from the centre. In terms of the measured voxel velocity, this effect can be understood as weighting of the velocities within the voxel where the central velocities contribute more strongly. Essentially, the effective slice thickness can be assumed to be smaller than the chosen one and thus even improving the accuracy of the measurement.

\subsubsection{Fluid Motion Artefact}

Another difficulty for the velocimetry sequence arises from the motion of the fluid during the acquisition of the $k$-space data. A common assumption for MRV experiments is that fluid displacements under the imaging part of the pulse sequence are small as compared to the typical voxel size (see e.g. Seymour et al. (1999)). In present work however, this assumption is not generally valid due to the high resolution used in the read direction. Fig. 5.10 shows snapshots of the deformation process of the initially selected slice shown in Fig. 5.8c in the case of the shear banding model fluid. The time range of $200 \mathrm{~ms}$ covered in the figure approximately concurs with a typical $k$-space sampling duration for the PGSE-RARE sequence in the present case. Considerable amounts of in- and outflow are observed in particular for the voxels close to the inner wall. In order to understand the motion effect better the 'velosim' program (section 4.1) has been used to model the PGSE-RARE sequence under these conditions. 

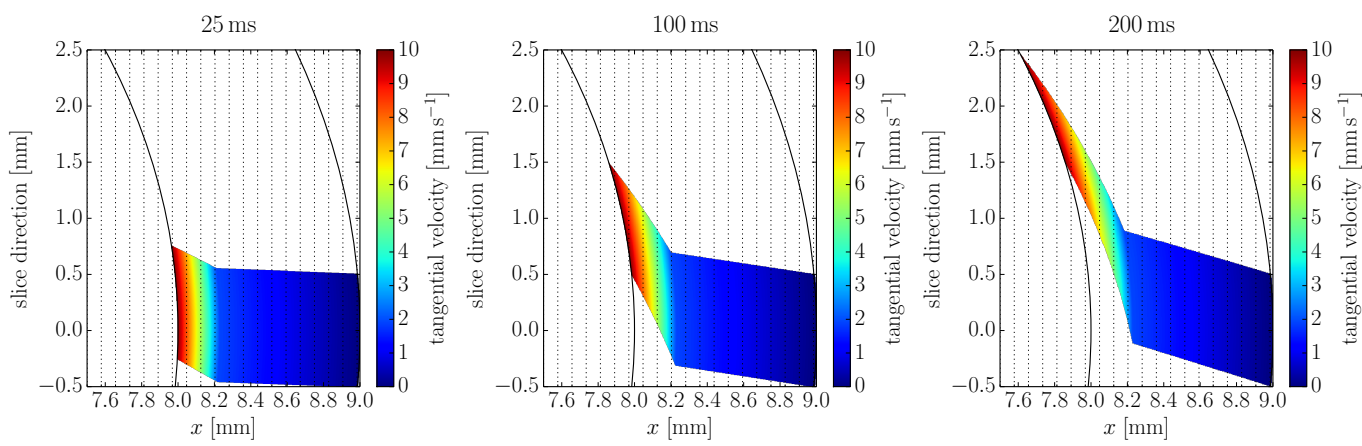

Fig. 5.10: Slice deformation at $\dot{\gamma}_{a}=10 \mathrm{~s}^{-1}$ for the shear banding model fluid $25 \mathrm{~ms}, 100 \mathrm{~ms}$ and $200 \mathrm{~ms}$ after the PGSE slice selection. The times approximately correspond to the beginning, the middle and end of the $k$-space acquisition, respectively.

\subsubsection{Simulation Sequence}

In Fig. 5.11 the modified PGSE-RARE used in the NMR simulation is shown. In comparison to the sequence of the experiment (Fig. 5.3), the slice selective soft pulses have been replaced by perfect $180^{\circ}$ hard pulses. This was necessary since the treatment of soft pulses is not included in the simulation program. As described earlier, the selection of the active volume is implicitly given by the reduction of the simulation grid to the desired volume. Furthermore, the transverse magnetization component perpendicular to the rotation axis of the RARE $180^{\circ}$ pulse was set to zero after the PGSE part of the sequence indicated by the 'Filter' element in the pulse sequence. This was done to avoid the possible appearance of a 'ghost' image in the case of the linear $k$-space scheme (see section 2.2.3.1). Such a filter could also be implemented in a real pulse sequence by the addition of two $90^{\circ}$ pulses with a gradient spoiling period in between as demonstrated by Ahola et al. (2006). Gradient spoiling refers to the act of deliberately dephasing magnetization by applying a (strong) gradient, without undoing this particular dephasing at a later stage. Apart from the differences just presented and some small technical details, such as gradient ramping, the simulation sequence is essentially identical to the one used for the experiments. In particular, the echo time $2 \tau$ is adjusted in the simulation by the insertion of an additional delay in the RARE loop so that it matches the echo time in the experiments for identical input parameters. The number of points in each dimension of the simulation grid $\left(N_{r}\right.$, $\left.N_{\varphi}, N_{z}\right)$ were $(50,50,100)$.

A typical simulation result is shown in Fig. 5.12. The field of view (FOV) was set to $20 \mathrm{~mm} \times 30 \mathrm{~mm}$ in the read phase $(x-z)$ imaging plane with a slice thickness of $1 \mathrm{~mm}$ in the slice direction $(y)$. A grid of $256 \times 32$ points was acquired resulting in a spatial resolution 


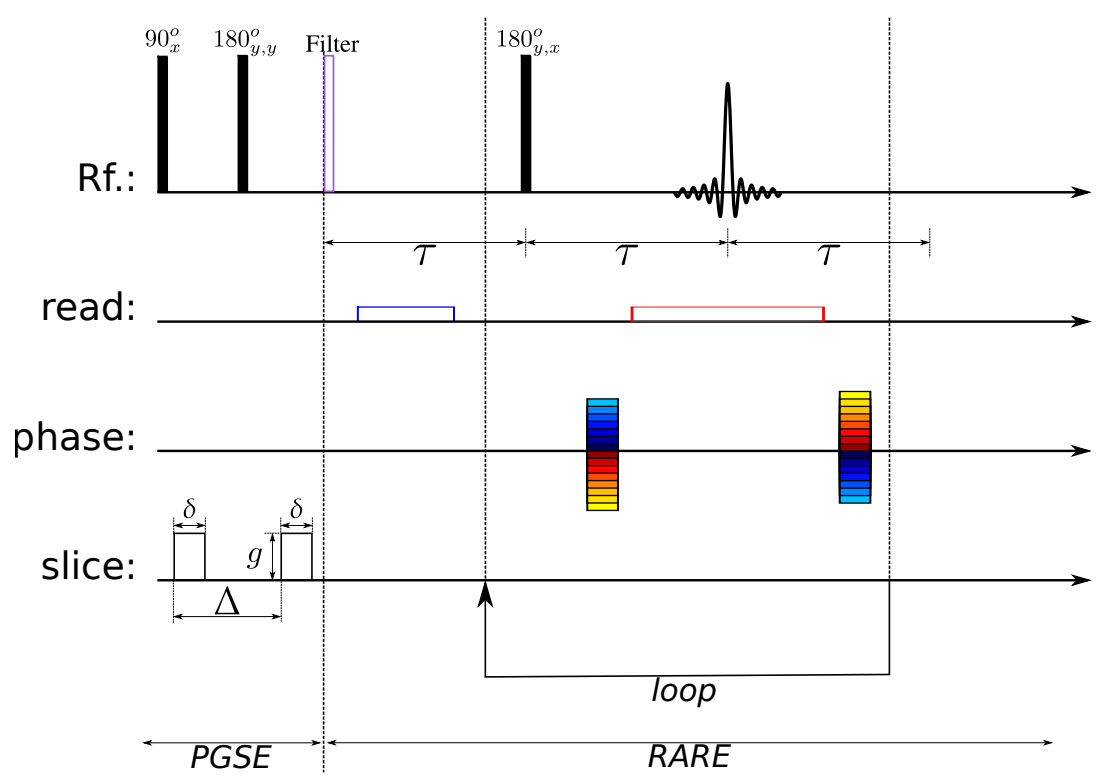

Fig. 5.11: PGSE-RARE sequence variant used in the 'velosim' program. In contrast to the sequence used for the experiment (Fig. 5.3) hard refocusing pulses are used and a 'Filter' element is added.

of about $80 \mu \mathrm{m} \times 1 \mathrm{~mm}$. The velocity encoding parameters $\delta=1 \mathrm{~ms}$ and $\Delta=20 \mathrm{~ms}$ were identical to the ones used in the real experiment throughout this work. The $2 \mathrm{D}$ velocity map was generated from four individual scans using two different encoding gradients ( 0 and $g \neq 0$ ) and phases for the RARE $180^{\circ}$ pulse according to Fig. 5.11. The height of the simulated Couette cell was $25 \mathrm{~mm}$. Otherwise, the dimensions were equal to those of Couette cell B. As mentioned in section 4.1, only one 'gap' is simulated due to the symmetry of the cylindrical Couette flow.

\subsubsection{Choice of $k$-space Scheme}

As mentioned in section 2.2.3.1, a commonly applied $k$-space trajectory for the RARE sequence is following a linear scheme (McRobbie et al. (2006)), where lines in $k$-space are acquired consecutively starting at the most negative (or positive) value in $k_{\text {phase }}$. This implies that the centre of $k$-space, which stores the main image features, is sampled in the middle of the acquisition period. Exemplified by the case of the shear banding model fluid, the initially selected slice volume might be considerably deformed at this point of the sequence (see Fig. 5.10b). Consequently artefacts might be introduced and velocities are mapped to "wrong" voxels in the resulting flow map. In order to reduce this motion 

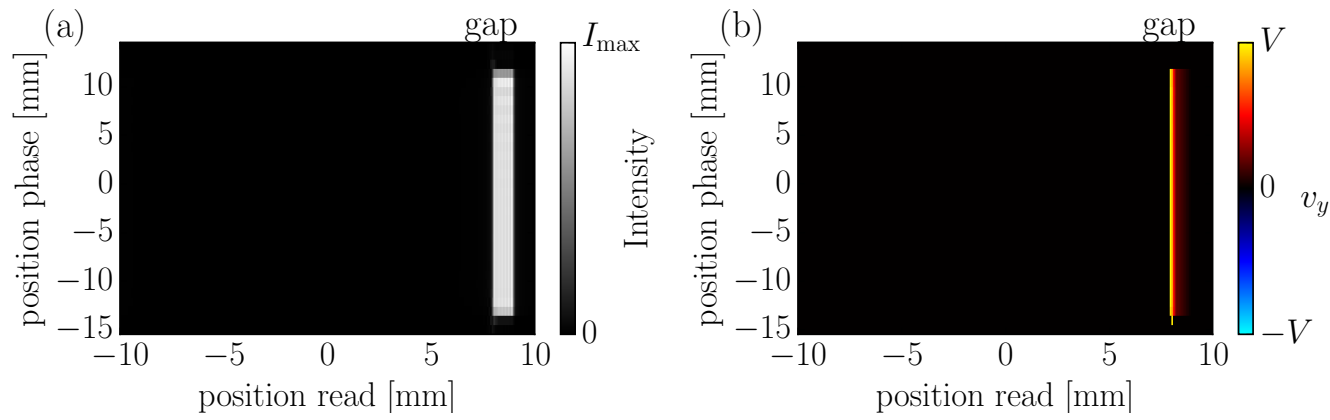

Fig. 5.12: Typical outcome of the $2 \mathrm{D}$ PGSE-RARE simulation: Magnitude (a) and flow (b) map. Read and phase correspond to $x$ and $z$ respectively as defined in Fig. 5.4. The field of view is $20 \mathrm{~mm}$ in read and $30 \mathrm{~mm}$ in the phase direction. The height of the simulation cell is $25 \mathrm{~mm}$. The values $N_{r}, N_{\varphi}$ and $N_{z}$, which are defining the simulation grid, were set to 50, 50 and 100, respectively.

effect the application of a centric scheme (Fig. 5.3) is recommended, where the centre of $k$-space is sampled at the beginning of the acquisition window.

To support this claim, 2D flow maps at different $\dot{\gamma}_{a}$ for the linear and centric scheme have been generated using the simulation sequence described in section 5.2.3.1. Taking an average over the phase direction over the range of the Couette cell $(25 \mathrm{~mm})$ results in $1 \mathrm{D}$ velocity profiles as shown in Fig. 5.13a at two different applied shear rates $\dot{\gamma}_{a}=10 \mathrm{~s}^{-1}$ and $20 \mathrm{~s}^{-1}$. Furthermore, the linear and the centric $k$-space scheme are compared in Fig. 5.13a. It is obvious that in particular for the high shear rate band velocity values recorded with the linear scheme are below the expectation, whereas the profile is accurately captured by the centric scheme. The underestimation of voxel velocities for the linear scheme can be explained by the following consideration. Due to the circular nature of the flow in the Couette cell, the original symmetry in the slice direction for the selected slice is broken and a net displacement towards the origin in the $x$-direction of the fluid contained in the slice can be observed during the simulation period (see Fig. 5.10). This net displacement causes a decrease of the mean tangential velocity for the velocity encoded fluid portion in each voxel volume, as $v_{\varphi}$ generally decreases with increasing $|x|$ for the given flow profile. In particular, voxels close to the inner wall, where a high velocity gradient is present, are considerably affected by this fluid migration. If one assumes that the main contribution to the measured (tangential) velocity value for a certain voxel comes from the average during the acquisition of the $k$-space centre, then a later sampling of this $k$-space region will result in a lower apparent velocity for that voxel. This explains why an underestimation of velocity is observed for the linear but not for the centric $k$-space scheme. Also, additional voxels below the gap radius of $8 \mathrm{~mm}$ exhibit non-zero velocity for 
the linear $k$-space trajectory similar to what has been observed in the $4 \mathrm{~mm}$ slice thickness case (sec. 5.2.2.1). Again these might give a wrong impression of the gap extent as well as the size of the high shear rate band.
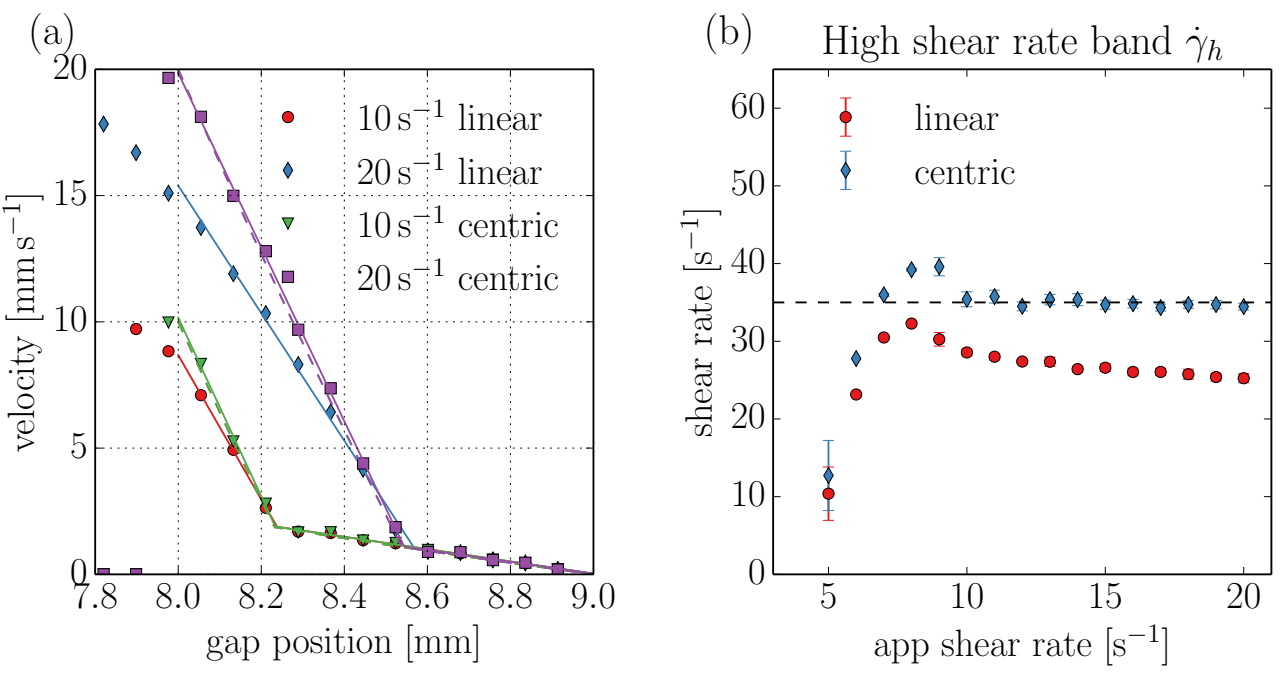

Fig. 5.13: Flow during the RARE sequence can affect velocity measurements. (a) Spatially averaged velocity profiles for linear and centric $k$-space scheme at two values of $\dot{\gamma}_{a}$ obtained by NMR simulation using the model shear banding fluid. The piece-wise linear fits (solid lines) only include pixels that are fully situated in the gap range. Dashed lines correspond to the expected profile for each shear rate. (b) $\dot{\gamma}_{h}$ as a function of the applied shear rate, extracted by fitting of the spatially averaged velocity profiles for both $k$-space schemes.

As stated, high shear rate regions are especially affected due to the considerable velocity differences of neighbouring voxels. In Fig. 5.13b, values for $\dot{\gamma}_{h}$ for a range of applied shear rates $\dot{\gamma}_{a}$ are depicted. These values are extracted from piece-wise linear fits of the spatially averaged velocity profiles, as shown in Fig. 5.13a. Deviations from the expected value $\left(\dot{\gamma}_{2}=35 \mathrm{~s}^{-1}\right)$ at lower shear rates are mainly due to an insufficient resolution for resolving this band. For $\dot{\gamma}_{a} \geq 10 \mathrm{~s}^{-1}$ however, the centric scheme accurately produces the expected value for $\dot{\gamma}_{h}$ whereas considerable deviations (> 20\%) are observed for the linear scheme. Furthermore, these deviations increase with $\dot{\gamma}_{a}$ due to an increased effect of the fluid inand outflow.

Flow profiles obtained from simulations using a $4 \mathrm{~mm}$ slice are essentially equivalent to the ones presented in Fig. 5.8b. Therefore one can conclude that for thicker slices motion artefacts are not equally important as for thin slices where the main complication lies in the slice deformation. It should also be clear that motion artefacts would have a different signature if for example the outer cylinder was rotated instead of the inner. Then one 
would expect an overestimation of velocities for the linear scheme as fast fluid would move into pixels positioned at lower $x$ values. A centric scheme would still be advantageous in this situation.

Furthermore, one can expect an increased quality in the flow measurement if only even echos are used to reconstruct $k$-space. These echoes are compensated for constant velocity flow in the read and phase direction and might decrease effects from gradient inhomogeneities. If only even echoes are used, it is advantageous to apply same phase gradient for each odd/even pair since this further minimizes additional phase contributions from gradient inhomogeneities and mismatches. However, the cost for the increase in the quality of the flow map is the reduction of resolution in the phase direction by a factor of two.

\subsubsection{Phase Cycling}

As discussed in section 2.2.3.1, the phase information of the transverse magnetization is lost under a CPMG train with imperfect refocusing pulses. In Section 5.2.2.2 it has been shown that the soft pulses used in the RARE loop generates a spectrum of rotation angles and can certainly not be regarded as perfect. Thus a velocity calculation is impeded if $k$-space is only scanned once for each $q$-step. Consequently, the images of two scans with perpendicular rotation axis for the soft pulses according to Fig. 5.3 are added up in order to restore the phase information. The phase of the PGSE $180^{\circ}$ pulse on the other hand is not altered for the two complementary images. Hence, the magnetization is always prepared in an equal manner before the imaging section. Note that this is in contrast to the work of Feindel and Callaghan (2010) and Brown and Callaghan (2011), where the phase of the PGSE refocusing pulse has been altered.

An alternative approach suggested by Shiko et al. (2012), where odd and even echoes are separated in order to maintain quadrature in a single run is applied in section 5.3.3.2 in conjunction with the high resolution method. Furthermore, it shall be mentioned that phase cycles where the phase of the refocusing pulse is switched during the CPMG train as a mean of phase conservation have not been considered for the present work. Such schemes are described in Shaka et al. (1988) and Gullion et al. (1990), for example.

\subsubsection{Determination of Gap Position}

Another challenge is the localization of the gap and wall position in the NMR flow map in the read direction. By the virtue of the Fourier transform, which is used to generate the 2D (phase) image, a Cartesian grid is defined by the imaging parameters such as the 
resolution and the field of view (see Fig. 2.10). In theory, this grid can directly be used to determine gap and wall positions in units of image pixels, if the dimensions of the geometry are known. However, experimental errors beyond the control of the experimenter might lead to spatial offsets and positional uncertainties in the image. Such errors can be caused for example by gradient inhomogeneities, lack of alignment of the gradient system and the Couette cell or imperfect shims. To account for these imperfections in the experimental setup two methods are proposed that allow for a more precise determination of the gap position in various situations.
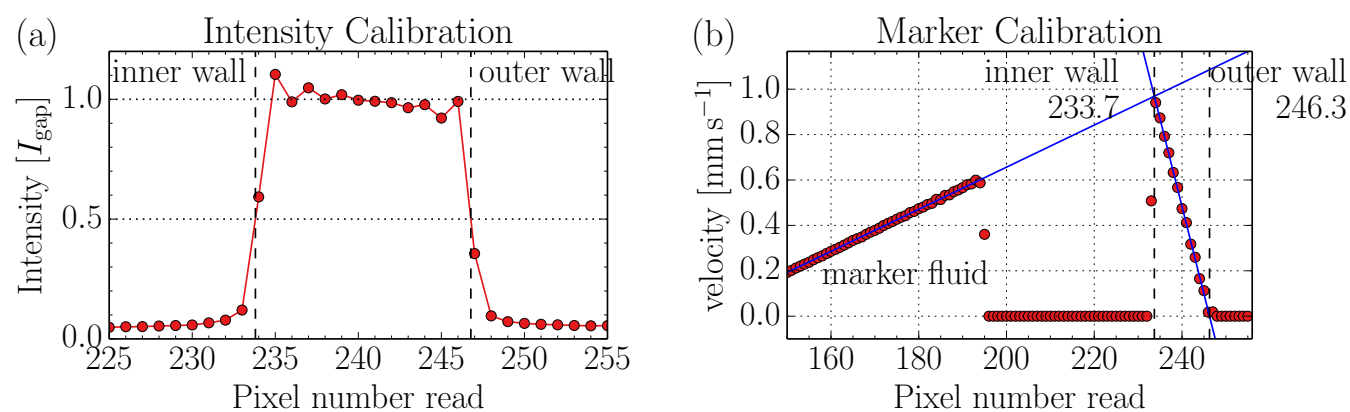

Fig. 5.14: Two methods for calibrating the gap location. Intensity calibration (a): The magnitude information of the NMR signal is used to determine the gap position. Intersections with the $50 \%$ threshold of the gap averaged signal intensity $I_{\text {gap }}$ are identified as the position of the wall-fluid interface. Marker calibration (b): Linear extrapolation of the marker fluid profile intersects the linear gap profile at the position of the inner wall. Outer wall position is given by the zero-intersect of the gap profile. The applied shear rate was $1 \mathrm{~s}^{-1}$. Dashed horizontal lines indicate the wall positions in both images. Please note that the data were extracted from the same experiment.

For the first method the magnitude information stored in the MRI image is used. If curvature effects are small, as is the case for a thin slice, and motion effects can be neglected, e.g. if the fluid is at rest, then the wall fluid interface is sharply defined in the read direction over the whole slice width (see Fig. 5.8c). In Fig. 5.14a an example of a signal intensity profile over one of the gaps in the MRI image is shown. In order to determine the location of the gap, pixel numbers in the grid matrix, where the signal has dropped to $50 \%$ of the gap averaged value $I_{\text {gap }}$, are computed and identified with the positions of the wall. This is justified by the fact, that one half of the voxel centered at this point would be within the gap volume, the other half outside and therefore the center of the voxel would be equivalent to the position of the fluid wall interface. The pixel position of the $50 \%$ threshold can be determined by linear interpolation of the corresponding pixel 
values for example. In the present case, the positions of the inner and outer wall in pixel units were 233.8 and 246.8 respectively, which results in a gap width of 13 pixel units. This is in good agreement with the expected value of 12.8 pixel units, given by an actual gap width of $1 \mathrm{~mm}$ in real space and a pixel resolution of about $78 \mu \mathrm{m}$ in the image.

The second method makes use of the flow map directly, while relying on a known linear velocity profile over the gap. It is inspired by the calibration technique described in Manneville et al. (2004) used for an ultrasound velocimetry technique, which is discussed in section 6.2.2 in general terms. The procedure of the second method is illustrated in Fig. 5.14b. Both the marker fluid and the gap profile are fitted with linear functions (solid lines). Using geometrical considerations, it is clear that the linear extrapolation of the marker fluid profile is intersecting the linear gap profile $v_{\text {gap }}(p)$ exactly at the outer wall of the inner cylinder if no slip is present. $p$ shall denote the position in the read direction in pixel units. The pixel position of the outer wall $p_{\text {out }}$, on the other hand, is then given by $v_{\text {gap }}\left(p_{\text {out }}\right)=0$. In the case of WLM solutions, slip-free linear profiles are expected if the applied shear rate $\dot{\gamma}_{a}$ is below the shear banding transition, which is starting approximately at $\dot{\gamma}_{1}$, and the geometrical ratio $\Lambda$ (Eq. (3.11)) is small. The profile in Fig. 5.14b was recorded at $\dot{\gamma}_{a}=1 \mathrm{~s}^{-1}$ for a WLM solution with $\dot{\gamma}_{1} \approx 3 \mathrm{~s}^{-1}$ confined to cell B. For the second method it is usually necessary to correct for a small velocity offset in the flow map, typically smaller than $0.1 \mathrm{~mm} \mathrm{~s}^{-1}$ originating from experimental imperfections. To calculate this offset, the pixel position of the Couette cell centre $p_{\text {centre }}$ is determined using the magnitude information of the marker fluid. The velocity offset itself is then given by the measured velocity at $p_{\text {centre}}$.

Please note that both methods only work, if the magnitude and the velocity information are not scaled by curvature or motion effects that are described in the previous sections. For a thick slice, e.g. $4 \mathrm{~mm}$ in the case of cell B, the wall fluid interface is not well defined in the read direction throughout the slice depth (Fig. 5.8a) and therefore intensity calibration (method 1) would not yield good results. Also the assumptions made for the marker calibration (method 2), would not be valid anymore. Furthermore it shall be pointed out, that the accuracy of the calibration depends on the signal-to-noise ratio of the data used. In order to increase the SNR, the intensity and flow profiles can be averaged in the phase direction if the profiles are expected to be invariant in that direction. Also averaging over a certain number of flow maps, that were acquired consecutively for the same flow conditions, leads to an increase in quality. For example, both profiles in Fig. 5.14 are averaged over 14 profiles in the phase direction and 100 individual 2D flow maps. 


\subsubsection{Measurement Timescales and Relaxation}

There are several important timescales involved in the measurement process of the PGSERARE sequence. The first one is the observation time $\Delta$ over which velocity information is accumulated and which approximately equals to the duration of the PGSE part. As mentioned earlier, $\Delta=20 \mathrm{~ms}$ for all NMR velocimetry experiments presented here. The second important time scale is the duration of the $k$-space acquisition process (RARE), which takes about $200 \mathrm{~ms}$ for typical imaging parameters. Under this period the deformation of the initially selected slice can lead to spurious phase contributions causing motion artefacts described in section 5.2.3. A single excitation followed by a PGSE encoding and a $k$-space traversal shall be referred to as a (single) 'scan'. The time needed for a scan is given by sum of the PGSE and RARE part duration and shall be denoted as $t_{\text {scan }}$. Usually at least four excitations/scans are necessary to construct a single velocity map. Thus, the repetition between consecutive excitations $T_{\mathrm{R}, \text { scan }}$ multiplied by the number of scans $N_{\text {scan }}$ determines the experimental repetition time $T_{\mathrm{R}}$, which is the third important timescale. Commonly, a series of $N_{f}$ flow maps is acquired consecutively yielding the total time of an experiment $T_{t}=N_{f} T_{\mathrm{R}}$. Consequently, $T_{R}$ also defines the temporal resolution of the method. It should be clear however, that the velocity measured in a single map is not an average over the whole period $T_{R}$. It is rather a velocity average over the $N_{\text {scan }} \Delta$-periods of each single scan. This distinction is important in the case of fluctuative systems as will be outlined in section 6.3.2.3.

With regards to the transverse relaxation, it should be noted that $T_{2}$ of the studied substance should be in the order of or larger than $t_{\text {scan }}$. Otherwise individual lines in $k$ space will be significantly weighted by the $T_{2}$ relaxation process and might be considerably noise inflicted if sampled at a later stage during the $k$-space traversal. Overall, this would deteriorate the quality of the image and the flow map. In terms of longitudinal relaxation, it is important to realize that the effective relaxation period is given by the difference of $T_{\mathrm{R} \text {,scan }}$ and $t_{\mathrm{scan}}$ for the spin system of interest. Again, $T_{1}$ should be in the order of this difference in order to ensure a sufficient initial magnetization for subsequent scans. By 'spin system of interest' the whole volume selected by the RARE slice is meant. This volume is constantly disturbed by the application of refocusing pulses under the $k$-space traversal of duration $t_{\text {scan }}$. This does also imply, that inflow of fresh undisturbed spins into the active region is not possible due to the circular motion of the fluid in the Couette cell.

In contrast to the present work, Feindel and Callaghan (2010) used an excitation pulse with a rotation angle of only $60^{\circ}$. In the case of the single slice selection scheme, which is 
used in the aforementioned reference, this can be advantageous, as spins that are outside the active region during a certain scan are only affected by the initial $60^{\circ}$ hard pulse, but not by the subsequent refocusing pulses during $t_{\text {scan }}$. Consequently, these spins can relax over a period of $T_{\mathrm{R} \text {,scan }}$ and might enter the active volume for the next scan bearing 'fresher magnetization'. However, as this scheme of achieving a more relaxed spin system is not applicable to the double slice selection method, the excitation angle was set to $90^{\circ}$ throughout all experiments in this work.

\subsection{Experimental}

\subsubsection{Validation}

For the experimental validation of the considerations outlined in section 5.2 a wormlike micellar solution is used in conjunction with Couette cell B. The WLM solution itself shall be characterized and studied in chapter 6 . Presently, only the following characteristics are of importance. The solution exhibits a shear banding transition at $\dot{\gamma}_{1} \approx 3 \mathrm{~s}^{-1}$ at a temperature of $25^{\circ} \mathrm{C}$, which is set for the experiments. The velocity gradient in the high shear rate band $\dot{\gamma}_{h}$ is not fixed with a value of $10 \mathrm{~s}^{-1}-50 \mathrm{~s}^{-1}$ depending on the value of $\dot{\gamma}_{a}$. The shear rate in the high viscosity band $\dot{\gamma}_{l}$ is approximately equal to $\dot{\gamma}_{1}$. Furthermore, slip is expected at the inner rotating wall.

For each shear flow experiment a series of 450 2D flow maps have been recorded over a period of $900 \mathrm{~s}$. The field of view has been set to $20 \mathrm{~mm} \times 25 \mathrm{~mm}$ in the read phase $(x-z)$ imaging plane. In the case of the centric $k$-space scheme a grid of $256 \times 16$ points was acquired, where only the even echoes where used. For the linear scheme the grid has a size of $256 \times 32$ points, however, all echoes where used. Thus the total $k$-space sampling time was equivalent for both schemes. After the acquisition the flow maps are averaged over time and the phase direction yielding 1D velocity profiles. Spurious profiles at the edge of the field of view in the phase direction were not included in the average. Each of the 1D profiles was fitted with a piece-wise linear function assuming two velocity gradients for a more quantitative analysis.

Slice Thickness PGSE As a first step, the thickness of the PGSE slice was varied in order to verify the depth effect described in section 5.2.2.1. In Fig. 5.15a the spatially and temporally averaged velocity profiles at a shear rate of $16 \mathrm{~s}^{-1}$ are shown for various slice thicknesses using the centric $k$-space scheme. In particular for the high shear rate band, huge differences in the recorded values for each data point are obvious. For the thickest 
slice of $8 \mathrm{~mm}$ even differences in the low shear rate band are obvious. In agreement with the theoretical considerations, the thinnest slice $(1 \mathrm{~mm})$ yields the highest measured velocity values. Judging from the converging behaviour towards thinner slices, a thickness of $1 \mathrm{~mm}$ seems close to an accurate description of the radial velocity profile. This claim is supported by Fig. $5.15 \mathrm{~b}$ where the slopes $\dot{\gamma}_{h}$ extracted from the piece-wise linear fits are plotted for various applied shear rates and slice thicknesses. The values obtained for thicknesses of $4 \mathrm{~mm}$ and $8 \mathrm{~mm}$ are far below the $1 \mathrm{~mm}$ case for all applied shear rates. In the case of the $2 \mathrm{~mm}$ slice, deviations from the $1 \mathrm{~mm}$ slice are considerably smaller. Thus it can be assumed that the $1 \mathrm{~mm}$ slice thickness case is close to an accurate description of the average flow profile. This observation is inline with the theoretical considerations from section 5.2.2.1. It shall be mentioned that even lower slice thicknesses are possible. However, these suffer from an even lower SNR and do not offer a huge gain in accuracy.

$\boldsymbol{k}$-space Scheme The effect of the $k$-space scheme is shown in Fig. 5.16. As expected, the linear scheme leads to an underestimation of velocities and deviates considerably from the centric scheme for both the $1 \mathrm{~mm}$ and $2 \mathrm{~mm}$ slices. As observed with simulation, the underestimation is almost independent of the slice thickness. It shall be noted however, that part of the deviations might also be related to the fact that all echoes have been used in the linear case, as the flow maps are of poorer quality.

Gap Calibration Finally the performance of the calibration method has been tested by comparing calibrated profiles for gap1 and gap2. In Fig. 5.17a the gap boundaries originating from an intensity calibration have been used. It can be observed that the profiles from both gaps agree well at each value of $\dot{\gamma}_{a}$, emphasized by piece-wise linear fits. The velocities at the inner wall $v(0)$ are slightly different for gap1 and gap2 at applied shear rates of $14 \mathrm{~s}^{-1}$ and $20 \mathrm{~s}^{-1}$. This is not expected due the symmetry of the shearing geometry and hints towards additional effects that are not accounted for by the calibration method. In the case of the marker calibration (Fig. 5.17b) on the other hand, the velocities at the inner wall agree slightly better, in particular for the $20 \mathrm{~s}^{-1}$ case. There the value for $v(0)$ is also smaller.

In summary, both methods achieve a good agreement for the two gaps, indicating that the real gap range in the flow map is determined with good accuracy. The marker fluid method is slightly superior in terms of equivalence of both gaps. However, the application of this method relies on the presence of a marker fluid, and velocity offsets have to be corrected in order to achieve a meaningful calibration. Furthermore, the performance of the calibration procedure should not be overestimated. A remaining uncertainty of 

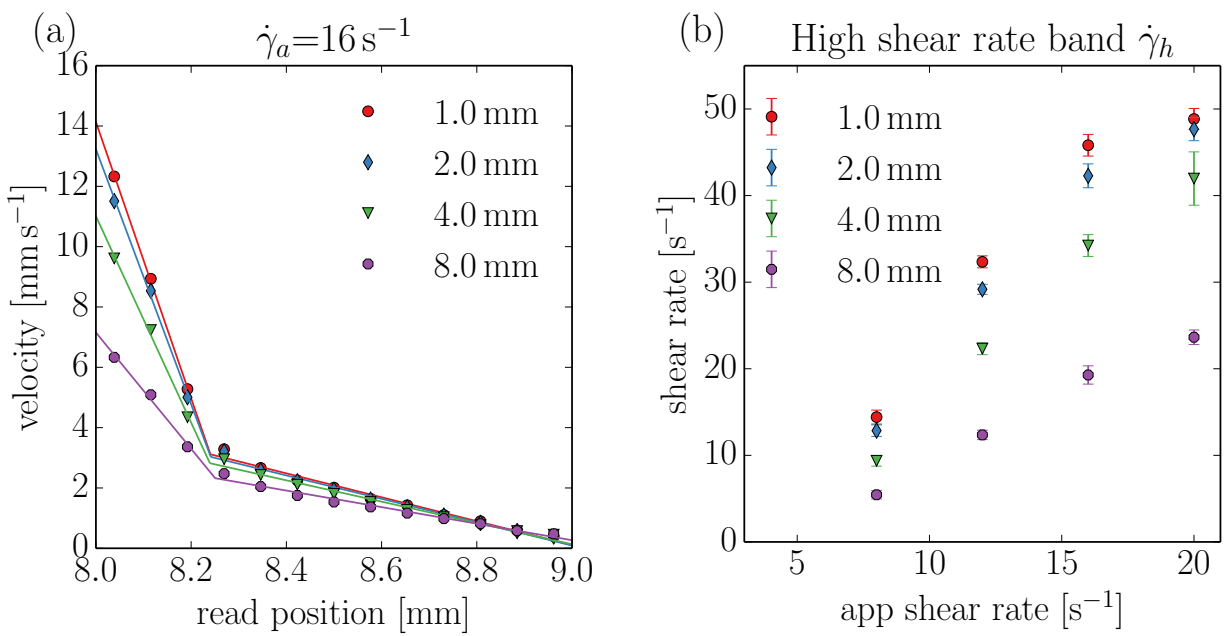

Fig. 5.15: (a) Spatially and temporally averaged velocity profiles for a shear banding fluid acquired with the PGSE-RARE sequence from Fig. 5.3 for various slice thicknesses. The solid lines correspond to piece-wise linear fits assuming two different slopes. (b) The slope of the high shear (velocity gradient) band as a function of the applied shear rate for various slice thicknesses. Errorbars are given by the uncertainty from the fitting routine.
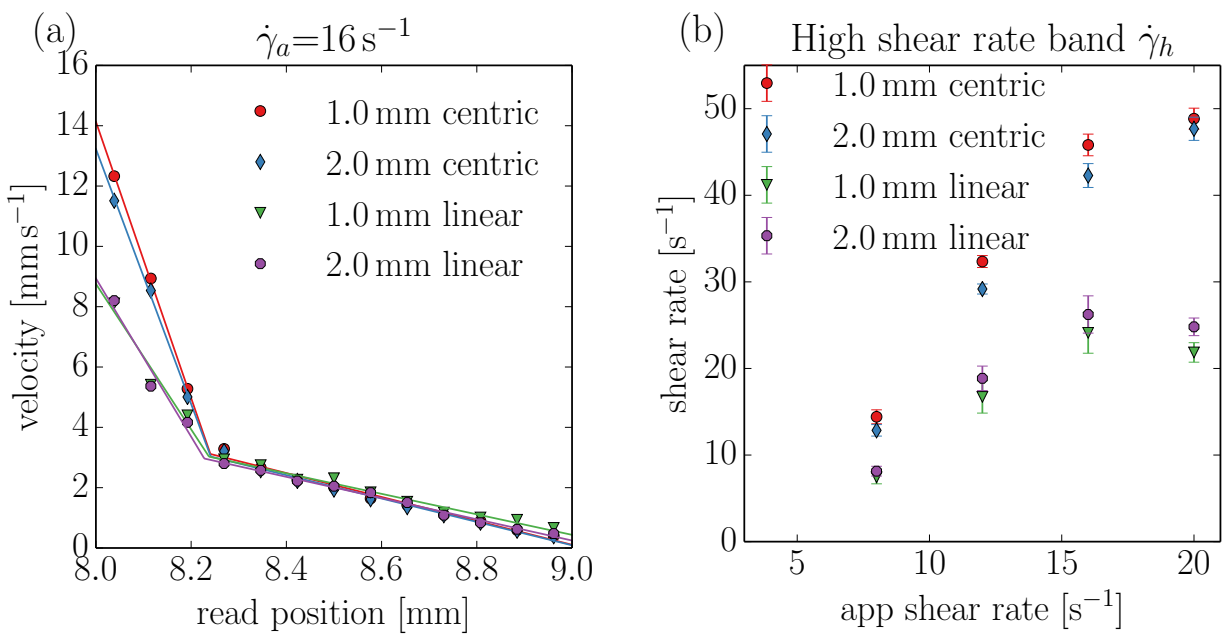

Fig. 5.16: Same as Fig. 5.15, but for different $k$-space schemes. 
about half a pixel width with regards to the absolute wall position for either method can be regarded as realistic and should be taken into account in the analysis of the flow data. Finally, it shall be emphasized that the calibration has to be done under the same conditions as the experiment to which the calibration should be applied. Results would be inferior if the calibration was, for example, done with a water sample while the actual experiment measures a WLM solution. Small differences in the setup, such as the shim or the relative position of gradient system and shear geometry, can induce spatial offsets, which cannot be controlled easily.
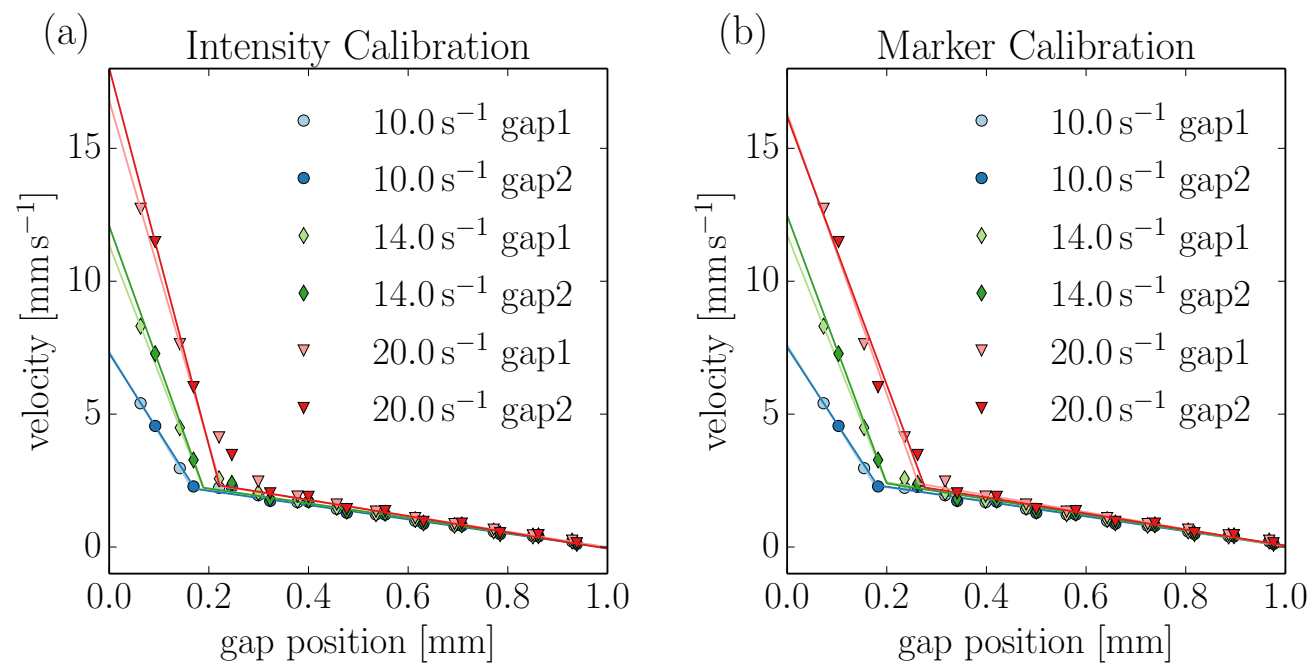

Fig. 5.17: Averaged gap velocity profiles for different $\dot{\gamma}_{a}$ from intensity (a) and marker (b) calibration. The solid lines are piece-wise linear fits assuming two velocity gradients. Note that the inner wall position has been chosen as the origin for both gaps.

\subsubsection{Centric Scheme and Structures in the Phase Direction}

While the centric $k$-space scheme is of advantage in accurately determining the velocity profile over the gap of a Couette cell, it might be less suitable for resolving structures in the phase direction, which are of great interest in complex fluid flow. The outer $k$-space region is more subjected to relaxation effects as compared to the linear scheme, where the trajectory starts at one edge of $k$-space. Consequently finer image details, which are encoded in the outer $k$-space region, could be suppressed. In order to test if structures in the direction of the imaging phase gradient are still resolvable using the centric scheme, Teflon tape has been wrapped around the inner cylinder for cell A creating a flow feature in 
the phase direction (see Fig. 5.4a). The tape increased the inner radius by approximately $0.5 \mathrm{~mm}$ and had a height of about $5 \mathrm{~mm}$, which is resolvable with a typical voxel length of $1.5 \mathrm{~mm}$ in the phase direction. The imaging fluid was distilled water with $0.15 \mathrm{mM} \mathrm{GdCl}_{3}$, which is also used for preparing the WLM solution. The corresponding values for the relaxation times $T_{1}$ and $T_{2}$ were measured to be around $500 \mathrm{~ms}$ and $350 \mathrm{~ms}$, respectively. Using a WLM solution is not preferable for this experiment as flow instabilities might occur due to the increased shear rate at the tape position.

A series of 50 2D flow maps were recorded using a slice thickness of $1 \mathrm{~mm}$ and the centric $k$-space scheme at an applied shear rate of $10 \mathrm{~s}^{-1}$. Apart from adjusting the FOV in the read direction to match the wider diameter of cell $\mathrm{A}$, acquisition parameters were essentially the same as in section 5.3.1. In particular, the scan repetition time $T_{\mathrm{R} \text {,scan }}$ was set to $0.5 \mathrm{~s}$. In Fig. 5.18a a single flow map out of the series is depicted. The effect of the Teflon tape on the fluid flow can clearly be seen for both gaps in the flow map. The $1 \mathrm{D}$ profiles in Fig. $5.18 \mathrm{~b}$ show that the fluid shear rate is altered at the position of the Teflon ring in the phase direction. This is expected due to the reduced gap width at this spot. Furthermore a slightly higher fluid velocity close to the inner wall, caused by the increased inner radius, can be confirmed at this position. Therefore one can conclude that the centric scheme preserves information in the phase direction.
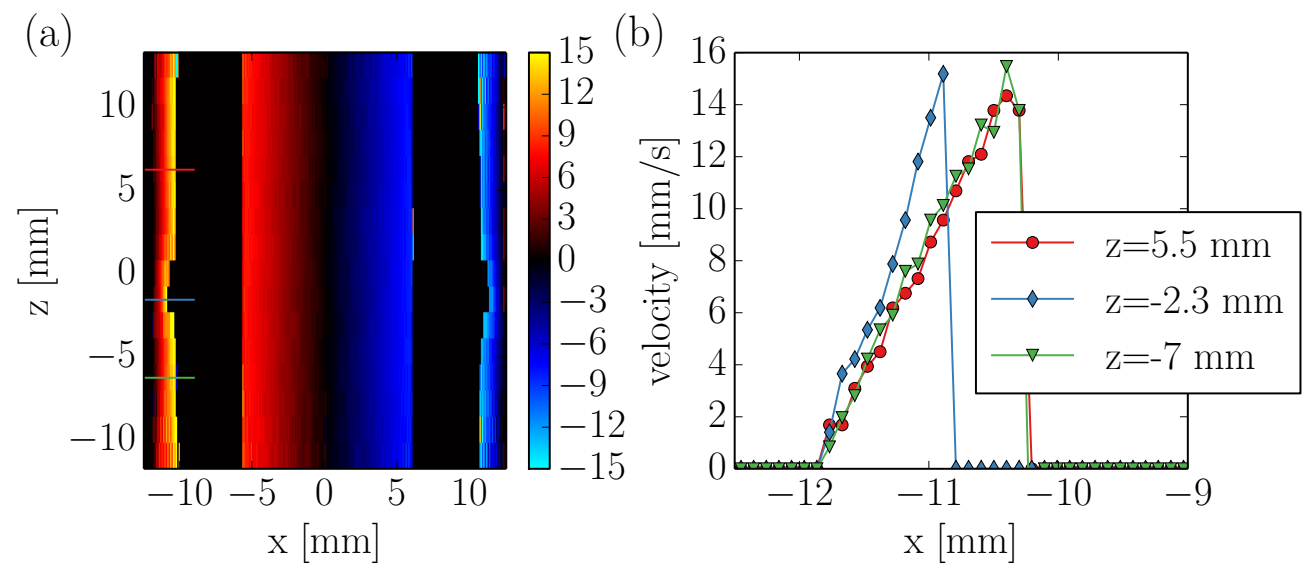

Fig. 5.18: (a) Single 2D NMR flow field from a series of 50 individual velocity maps for Couette cell A (without spurious artefacts). Teflon tape wrapped around the inner cylinder at $z \approx-2 \mathrm{~mm}$ creating a ring of about $5 \mathrm{~mm}$ height and $0.5 \mathrm{~mm}$ thickness served as a flow feature in the phase direction. Velocities between the two gaps are from a marker fluid undergoing rigid body rotation. (b) Flow profiles at different positions in the phase direction. 


\subsubsection{High Resolution Method}

Accurately imaging the velocity profile over the small gap extent of the Couette cells is a challenge for the fast imaging sequence. Given a typical resolution of about $100 \mathrm{\mu m}$ in the gap direction commonly used for the PGSE-RARE sequence in the case of Couette flow (Brown and Callaghan (2011); Feindel and Callaghan (2010)), only 10 data points are fully situated in the gap range in the case of cell B. Hence, flow features smaller than about $20 \%$ of the gap width $(200 \mu \mathrm{m})$ are difficult to capture quantitatively. A relatively small high shear rate band, in the case of wormlike micellar solution is a good example as slope $\dot{\gamma}_{h}$ and proportion $\alpha_{h}$ cannot be determined with great accuracy if only one voxel is fully positioned in this part of the flow profile. Therefore an increase of data points for the gap profile is desirable as these structures could become resolvable. A simple extension of the read acquisition in order to increase the resolution is usually not feasible, as it makes the sequence more prone to relaxation effects and reduces the possible experimental repetition rate. Resolution can also be improved by increasing the spectral width in combination with a higher read gradient, while keeping the acquisition time constant. However, apart from a stronger diffusive attenuation, the sampling rate might be beyond the capabilities of the NMR spectrometer if a significant increase in resolution is desired. Yet, the latter problem can be circumvented by reducing the FOV in the read direction to e.g. twice the gap size, as less points in the $k$-space grid have to be acquired in this situation. The resulting fold-over problem caused by the then too small FOV could be tackled by one of the following options. The first one is the introduction of a third slice selection in the read direction which only selects one of the gaps in 2D image. Yet, implementation might be difficult (e.g. when to apply the slice selective pulse within the sequence) and additional artifacts could be introduced. As the second option a frequency offset for the read acquisition can be used, in order to shift the center of the FOV within one of the gaps. Consequently either analog or digital filters common to NMR spectrometers can be used to remove unwanted signal coming from outside the imaging region. Option two has been preferred due to the simpler implementation for the present work.

The remaining difficulty is the necessity to apply strong read gradients over an extended amount of time in order to achieve the desired resolution, if the acquisition period is not to be prolonged considerably as compared to the conventional method. For example, a read resolution of $16 \mu \mathrm{m}$ corresponds to a read gradient of approximately $0.6 \mathrm{~T} \mathrm{~m}^{-1}$ for typical imaging parameters. These imaging gradients can therefore cause a considerable diffusive attenuation of the NMR signal. At a temperature of approximately $25^{\circ} \mathrm{C}$, the free diffusion coefficient of the solvent water of the micellar solution was measured 


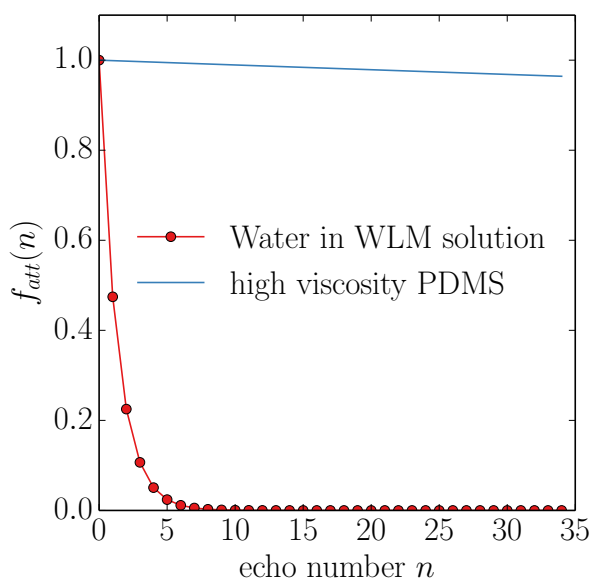

Fig. 5.19: Signal attenuation due to free diffusion according to the Stejskal-Tanner relation (Eq. (2.28)) for the water acting as solvent in a WLM solution and a high viscosity PDMS solution in the case of the high resolution PGSE-RARE sequence.

to be around $1.6 \times 10^{-9} \mathrm{~m}^{2} \mathrm{~s}^{-1}$, slightly lower than the expected value for pure water $\left(2.3 \times 10^{-9} \mathrm{~m}^{2} \mathrm{~s}^{-1}\right)$. The corresponding signal attenuation factor (Eq. (2.28)) is shown in Fig. 5.19 as a function of the echo number. The values for $\Delta$ and $\delta$ equate to the echo time $2 \tau(\approx 6 \mathrm{~ms})$ and the duration of the read gradient $(\approx 3 \mathrm{~ms})$, respectively. After only a few echoes, most of the signal is damped away due to the diffusion of the water molecules. At this point, it shall also be mentioned that the signal originating from the micelle surfactant is weak in comparison to the water signal and not suitable for the imaging process. Therefore the acquisition of a $2 \mathrm{D} k$-space under a CPMG train is not favourable in this situation. Instead, the ability to resolve structures in the phase direction has been given up in the case of the WLM solution for the high resolution method. This 1D high resolution method is described in more detail in section 5.3.3.1. On the other hand, for sufficiently small diffusion coefficients, diffusive attenuation is considerably decreased, as shown in Fig. 5.19 in the case of highly viscous polydimethylsiloxane (PDMS), where $D \approx 2 \times 10^{-12} \mathrm{~m}^{2} \mathrm{~s}^{-1}$. Hence, the potential of a high resolution $2 \mathrm{D}$ method is shown in section 5.3.3.2 in the case of high viscosity PDMS.

\subsubsection{High Resolution 1D}

As the ${ }^{1} \mathrm{H}$ water signal is damped below the noise level after only a few echoes due to diffusive attenuation in the case of high read gradients, the RARE loop is reduced to only 
two echoes whereof the second is used to construct a $1 \mathrm{D}$ velocity profile. The first echo is discarded, because of spurious signal contributions. The reduction of the pulse sequence allows for the acquisition of 128 data points in the read direction over a FOV of $2 \mathrm{~mm}$ resulting in a resolution of about $16 \mu \mathrm{m}$. Due to the lack of a CPMG train considerations regarding phase conservation and $k$-space scheme are less relevant.

Fig. 5.20a shows velocity profiles for doped water $\left(0.15 \mathrm{mM} \mathrm{GdCl}_{3}\right)$ sheared in Couette cell B at various rates $\dot{\gamma}_{a}$ recorded with the 1D high resolution technique. Each velocity profile is an average over 450 single flow maps that were recorded consecutively with $T_{\mathrm{R}, \mathrm{scan}}=0.5 \mathrm{~s}$. Note, that each flow map is still constructed from two $q$-step and phase cycle steps. The application of a phase cycle is not compulsory, as one can assume that phase information is conserved after two refocusing pulses. Yet, the two step phase cycle has the effect of an improved SNR. The expected linear profile is found for all applied shear rates. However, a slight underestimation of the fluid velocity is apparent for all values of $\dot{\gamma}_{a}$, as the velocity of the fluid at the inner wall at $x=8 \mathrm{~mm}$ is not matching the velocity of the inner wall $V=\dot{\gamma}_{a} d$. The presence of slip is not regarded as likely in the present situation. Hence, this effect is prescribed to experimental imperfection. The observed deviations are around $5 \%$ and thus regarded as acceptable for the method.

Velocity profiles in the case of the wormlike micellar solution acquired with the same imaging parameters as for the water are depicted in Fig. 5.20b. Here the average is taken over 60 profiles corresponding to a 120 s recording period. The important feature is that the build-up of a high shear rate portion in the velocity profile is captured with increasing shear rate. After observing a linear profile at $\dot{\gamma}_{a}=2 \mathrm{~s}^{-1}$ which is below the shear banding threshold a small high shear rate band of about $30 \mu \mathrm{m}$ width can be observed for an applied shear rate of $4 \mathrm{~s}^{-1}$. Eventually, the extent of the high shear rate portion increases to about $150 \mu \mathrm{m}$ for $\dot{\gamma}_{a}=10 \mathrm{~s}^{-1}$. Furthermore, the high point density over the gap allows the quantification of the velocity gradient in high shear rate region. With a conventional resolution of about $100 \mu \mathrm{m}$ this would not be possible. As shall be discussed in chapter 6 , the slope $\dot{\gamma}_{h}$ in and the proportion $\alpha_{h}$ of the high shear rate band are increasing with $\dot{\gamma}_{a}$, an observation that was not possible previously. Therefore, the high spatial resolution of the velocity maps makes a quantitative description of the shear banding process directly after the shear banding threshold possible.

Another positive aspect of the high resolution method is that the position of the Couette walls can be determined more accurately. As mentioned in section 5.2.5, an uncertainty of half a pixel width is a reasonable assumption for the calibration process. Thus the significant increase in resolution reduces the relative uncertainty. Finally it shall be mentioned that single velocity profiles suffer from poor SNR due to the high read gradient applied. 

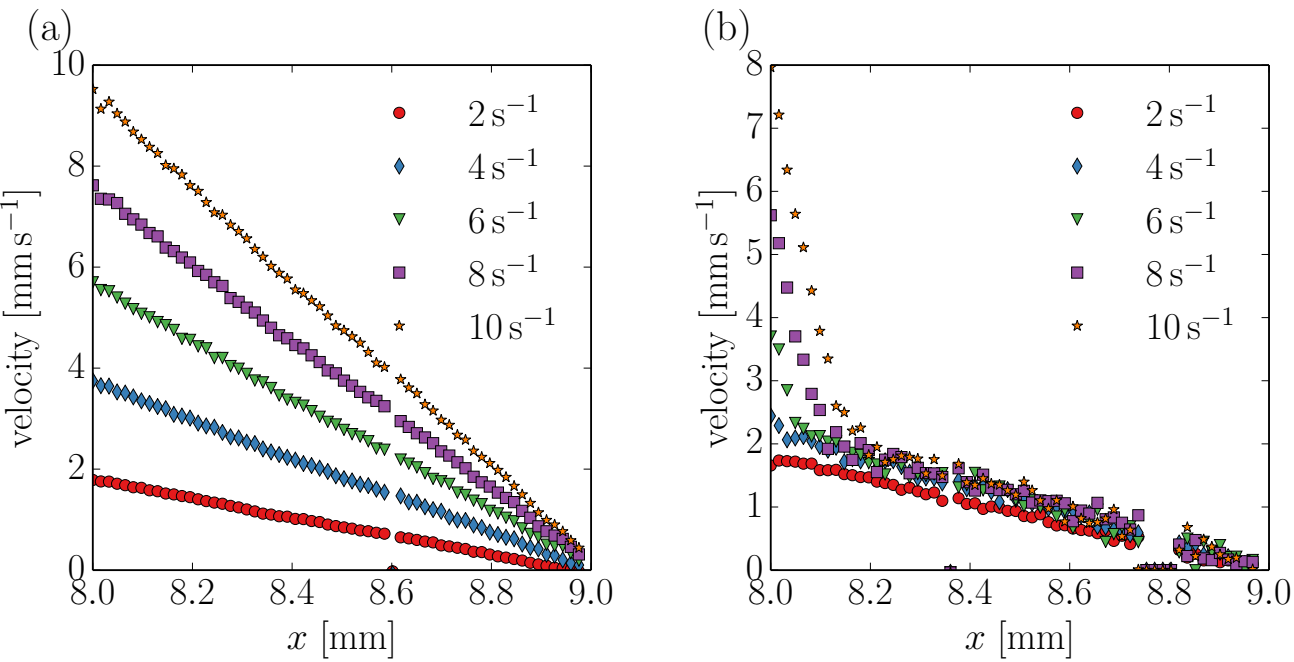

Fig. 5.20: (a) 1D HR velocity profiles of water for different applied shear rates. Each profile is an average over 450 single velocity profiles, that were acquired consecutively with a repetition time of $2 \mathrm{~s}$. (b) Same as (a) but for the wormlike micellar solution instead of water. Also, the average is taken over 60 profiles acquired over $120 \mathrm{~s}$. Because of the high resolution in the read direction, the banded flow can already be described quantitatively at an applied shear rate of $4 \mathrm{~s}^{-1}$ slightly after the shear banding transition. Please note, that the velocity in certain pixels was set to zero, as a velocity determination was not possible at these positions due to external (rf) signal perturbations.

Therefore, it is usually necessary to average over a certain number of flow maps to obtain the desired quality. An increase in PGSE slice thickness can result in a better SNR but the resulting flow map might suffer from decreased accuracy in the flow profile recorded. Thus, it is always necessary to find a compromise between SNR and temporal resolution for the method.

\subsubsection{High Resolution 2D}

As discussed already, the NMR water signal is attenuated significantly after only a few echoes in the RARE acquisition loop due to molecular diffusion for high read gradients. Thus the acquisition of a $2 \mathrm{D} k$-space is not possible if one relies on the signal of the ${ }^{1} \mathrm{H}$ water protons. Fluids exhibiting a low diffusion coefficient for the spin bearing nuclei on the other hand are suited for a $2 \mathrm{D}$ version of the high resolution method. To this end high viscosity PDMS $\left(60 \times 10^{3} \mathrm{cSt}\right)$ with $D \approx 2 \times 10^{-12} \mathrm{~m}^{2} \mathrm{~s}^{-1}$ has been filled in Couette cell $\mathrm{A}$ in order to show the $2 \mathrm{D}$ capabilities of the high resolution method. It shall 
be mentioned that PDMS is a Newtonian fluid and thus approximately linear velocity profiles are expected over the gap with no slip present.

The flow maps shown in Fig. 5.21 and Fig. 5.22 have a resolution of about $30 \mu \mathrm{m}$ in the read direction (FOV $2 \mathrm{~mm}$ ). In the phase direction 32 points are acquired over a range of $20 \mathrm{~mm}$ (all echoes have been used). The number of $q$-steps had to be increased to 8 in order to ensure a sufficient quality in the flow maps. Subsequently, velocity values have been obtained by the calculation of the propagator peak as described in Callaghan (2011). Due to the longer $T_{1}$ of the silicone oil $(\approx 1.5 \mathrm{~s})$ and in order to ensure a sufficient SNR the time between consecutive excitations was increased from $0.5 \mathrm{~s}$ to $7 \mathrm{~s}$ as compared to the previous experiments with doped water. This means that each flow map has been acquired over about $120 \mathrm{~s}$. Additionally, it shall be mentioned that the slice thickness has been increased to $2 \mathrm{~mm}$ to further increase the signal to noise ratio.

In Fig. 5.21 the centric $k$-space scheme has been applied to record the flow maps. For an applied shear rate of $6 \mathrm{~s}^{-1}$ the flow map meets the expectations with an increased shear rate in the Teflon band region. Also, the fluid velocities at the walls are in good agreement with the wall velocities for the chosen profiles. In particular the increased velocity for the Teflon band is recorded accurately. Slight irregularities observed at the top and bottom of the Teflon ring might be due to the fact that the ring edges are not very sharply defined. For higher shear rates (Fig. 5.21b,c) the flow map is more noise inflicted but still recovers the expected flow dynamics, in contrast to the linear $k$-space scheme shown in Fig. 5.22. There, only at the lowest shear rate of $6 \mathrm{~s}^{-1}$ a good agreement with the expected profile is found. For $\dot{\gamma}_{a}=12 \mathrm{~s}^{-1}$ the profiles already deviate considerably, most likely due to the fluid motion effect discussed in section 5.2.3. Also an increased amount of irregularities are found at the edges of the field of view in the phase direction in comparison to the centric scheme. For $20 \mathrm{~s}^{-1}$ the profiles are even more spurious. The irregularities might be explainable by an accumulation of spurious phase contributions due to the high imaging gradients and the fast motion of the fluid. This further underlines the advantage of applying a centric $k$-space.

Finally the method proposed by Shiko et al. (2012) (described in section 2.2.3.1) has been applied to the high resolution case. By making the dephasing read gradient unbalanced even and odd echoes can be separated. This allows maintenance of quadrature without the need to phase cycle and thus an increase in temporal resolution by a factor of 2 for the present case. However, if all other main experimental parameters are kept constant, a decrease in read resolution by a factor of 2 is encountered as well. In contrast to the conventional method this is not as severe in the high resolution case as still a sufficient number of gap pixels are present in the resulting flow profiles. 

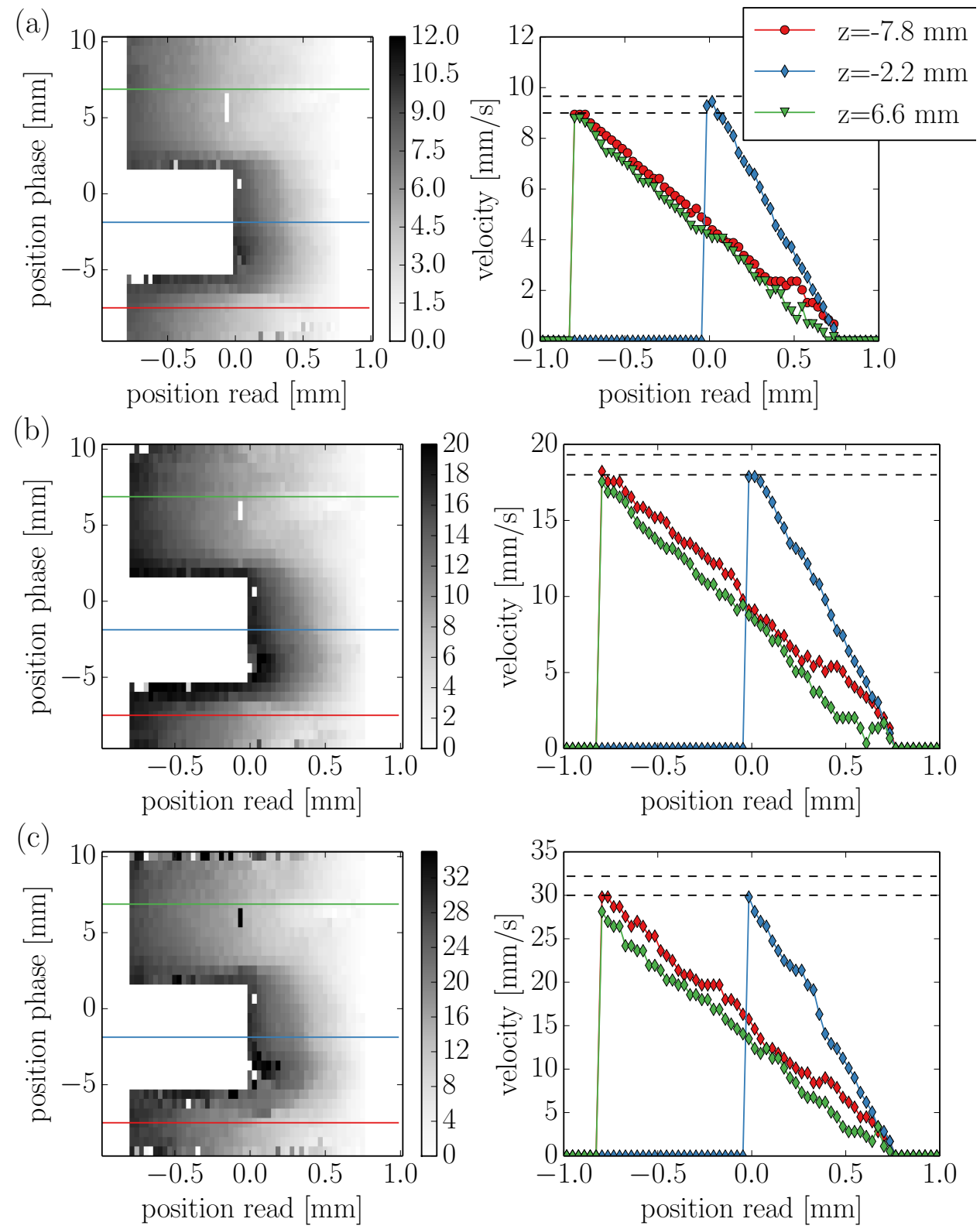

Fig. 5.21: 2D high resolution velocity maps for high viscosity PDMS at applied shear rates of $6 \mathrm{~s}^{-1}$ (a), $12 \mathrm{~s}^{-1}$ (b) and $20 \mathrm{~s}^{-1}$ (c) as recorded with the centric $k$-space scheme. The phase positions of the $1 \mathrm{D}$ velocity profiles are the same for all plots. The dashed horizontal lines mark the expected velocity of the inner wall and the Teflon band with the latter being higher. The velocity of pixels with low image intensity were set to zero.

In Fig. 5.23 a velocity map acquired over $60 \mathrm{~s}$ at a shear rate of $6 \mathrm{~s}^{-1}$ is depicted. Apart from an unbalanced read gradient and the lack of the second phase cycle step, all other 

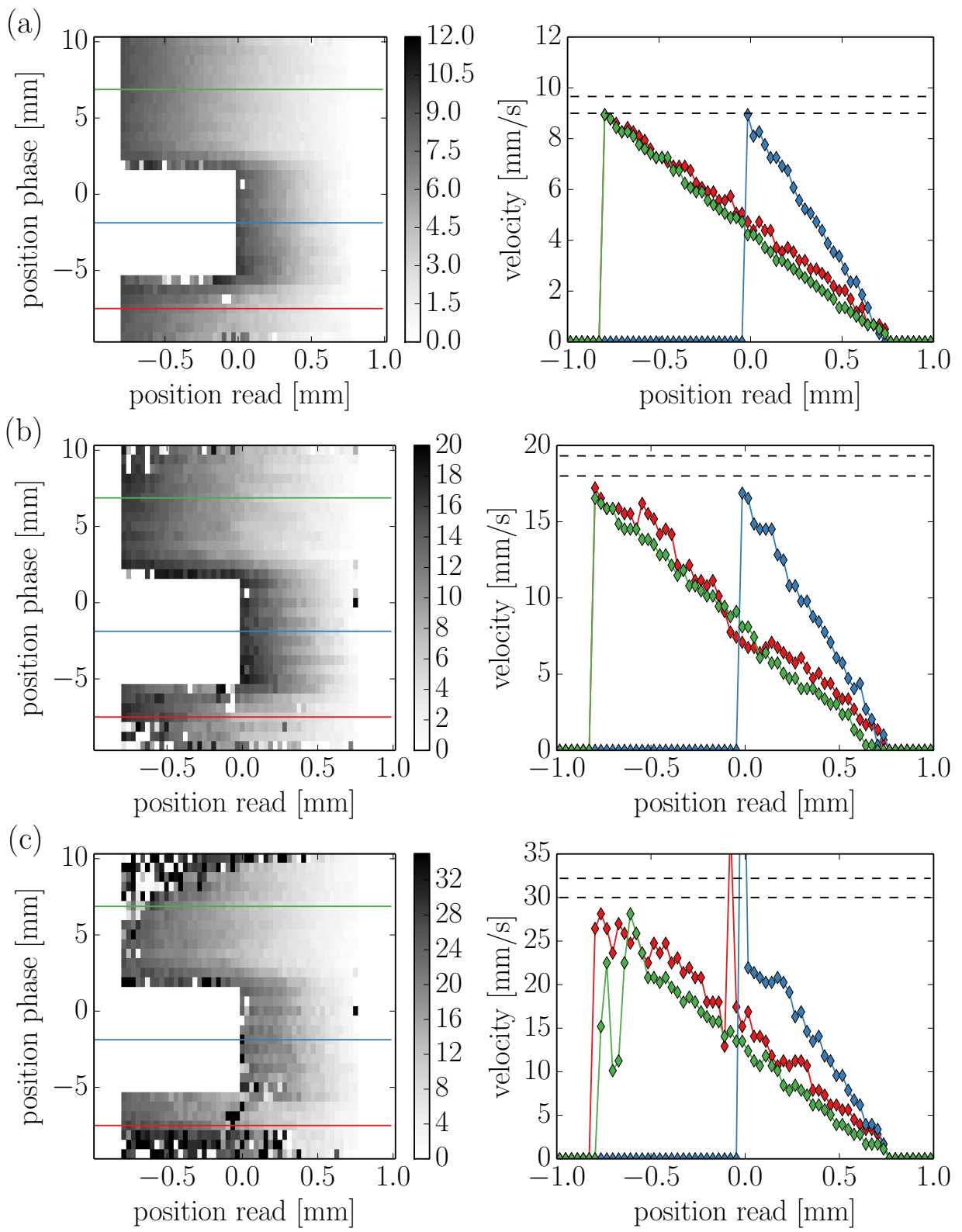

Fig. 5.22: Same as Fig. 5.21, but for the linear scheme.

main experimental parameters are equivalent to the flow map in Fig. 5.21a. Compared to the two step phase cycle, the profiles are of similar quality showing the potential of the method. It should be mentioned however, that only for the signal domain of the odd parity echoes the creation of a flow map was possible due to the application of the centric $k$-space scheme. The domain of the even parity echoes lacked the information on the central $k$ space line, which is sampled during the first echo of the RARE loop for the centric scheme, 
because no even parity echoes are generated for the first loop echo (see section 2.1.7). Due to the lack of the central line, the 'even parity echo' flow map was spurious and did not represent the flow field accurately. Hence, it was also not possible to average between the odd and even parity echo flow maps in order to improve the overall signal to noise ratio, as previously described in section 2.2.3.1. However, starting the $k$-space traversal after the first echo would amend this situation.
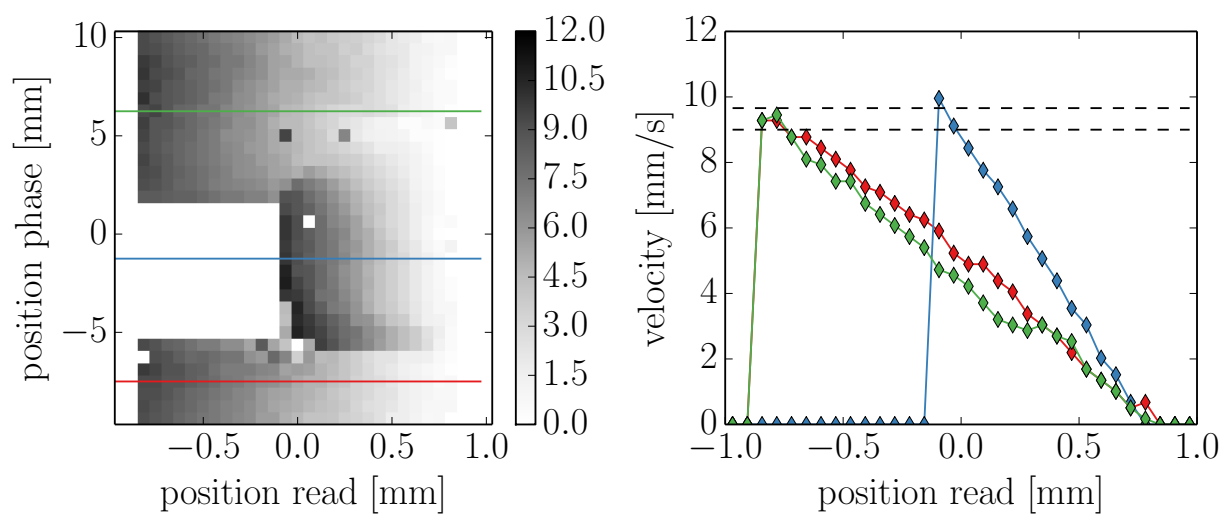

Fig. 5.23: Same parameters as in Fig. 5.21a, but recorded using an off-balanced read gradient, as described in Ref. Shiko et al. (2012). Due to the splitting of the coherence pathways the number of pixels in the read direction is reduced by a factor of two down to 32 .

\subsection{Conclusion}

A methodology has been developed that allows for more accurate velocity measurements in cylindrical Couette cells using a 2D PGSE-RARE sequence. It has been shown that the curvature of the shear geometry and the circular motion of the fluid in the cell introduces challenges to the imaging process that can be overcome by choosing appropriate experimental parameters. To this end, an optimal slice thickness was advised for the PGSE part of the pulse sequence that minimizes artefacts induced by the curvature of the geometry. It has been found that the right choice of slice thickness is particularly important for shear banded flow, where flow dynamics are otherwise not captured correctly. With regards to the slice selection for the RARE part, it has been outlined that a slice normal to the phase direction is a means of avoiding fold-over artefacts. Intensity scaling in the magnitude map and spurious velocity measurements on the edges of the field of view have been explained by a spectrum of effective tip angles induced by the RARE slice. These results 
were obtained with the help of the soft pulse simulation program described in section 4.2. Furthermore, the use of a centric $k$-space scheme for the imaging sequence was advised to reduce artefacts induced during the motion of the fluid under the $k$-space traversal. Both experiments and simulation show a clear advantage over a commonly applied linear scheme. Additionally, a scheme is advised for a more accurate localization of the gap position. For an improved control of thermal conditions during the NMR experiment a Couette cell recently developed in our research group by a fellow PhD student (Tim Brox) was introduced that can be used in conjunction with the Bruker temperature control system. Finally a method was presented that allows for a considerable increase in read resolution, which allows for a more accurate quantification of the flow dynamics. However, due to the strong gradients involved, the ability to resolve structures in the phase direction had to be given up in case where the spin bearing nuclei diffuse too fast. For substances with low diffusion constant on the other hand such as highly viscous PDMS, it was shown that 2D high resolution method can accurately capture a flow field depending both on radial and axial position.

In the following chapter this methodology shall be used to characterize the flow of a specific wormlike micellar solution in more detail than previously possible with NMR methods.

\section{Bibliography}

S. Ahola, J. Perlo, F. Casanova, S. Stapf, and B. Blümich. Multiecho sequence for velocity imaging in inhomogeneous rf fields. Journal of Magnetic Resonance, 182(1):143-151, September 2006. ISSN 1090-7807. doi: 10.1016/j.jmr.2006.06.017. URL http://www. sciencedirect.com/science/article/pii/S1090780706001765.

Jean-Fraņcois Berret, Grégoire Porte, and Jean-Paul Decruppe. Inhomogeneous shear flows of wormlike micelles:mA master dynamic phase diagram. Phys. Rev. E, 55(2): 1668-1676, February 1997. doi: 10.1103/PhysRevE.55.1668. URL http://link.aps. org/doi/10.1103/PhysRevE.55.1668.

Jennifer R. Brown and Paul T. Callaghan. Changing micellar order, lever rule behavior and spatio-temporal dynamics in shear-banding at the onset of the stress plateau. Soft Matter, 7(21):10472-10482, 2011. doi: 10.1039/C1SM06135F. URL http://dx.doi. org/10.1039/C1SM06135F. 
Paul T. Callaghan. Rheo-NMR: a new window on the rheology of complex fluids. In eMagRes, pages 737-750. John Wiley \& Sons, Ltd, 2007. ISBN 9780470034590. URL http://onlinelibrary.wiley.com/doi/10.1002/ 9780470034590.emrstm0470.pub2/abstract.

Paul T. Callaghan. Translational Dynamics and Magnetic Resonance:Principles of Pulsed Gradient Spin Echo NMR. Oxford University Press, September 2011. ISBN 9780191621048 .

Kirk Feindel and Paul Callaghan. Anomalous shear banding: multidimensional dynamics under fluctuating slip conditions. Rheologica Acta, 49(10):1003-1013, 2010. ISSN 00354511. URL http://dx.doi.org/10.1007/s00397-010-0470-9. 10.1007/s00397-0100470-9.

Petrik Galvosas and Paul T. Callaghan. Fast magnetic resonance imaging and velocimetry for liquids under high flow rates. Journal of Magnetic Resonance, 181(1):119-125, July 2006. ISSN 10907807. doi: 10.1016/j.jmr.2006.03.020. URL http://europepmc.org/ abstract/MED/16644252/reload=0; jsessionid=cYaR2x3QhLC6H9xa75Q0 . 12.

D. A. Grabowski and C. Schmidt. Simultaneous measurement of shear viscosity and director orientation of a Side-Chain Liquid-Crystalline polymer by Rheo-NMR. Macromolecules, 27(9):2632-2634, April 1994. ISSN 0024-9297. doi: 10.1021/ma00087a037. URL http://dx.doi.org/10.1021/ma00087a037.

Terry Gullion, David B Baker, and Mark S Conradi. New, compensated Carr-Purcell sequences. Journal of Magnetic Resonance (1969), 89(3):479-484, October 1990. ISSN 0022-2364. doi: 10.1016/0022-2364(90)90331-3. URL http://www. sciencedirect. com/science/article/pii/0022236490903313.

S. Manneville, L. Bécu, and A. Colin. High-frequency ultrasonic speckle velocimetry in sheared complex fluids. The European Physical Journal - Applied Physics, 28(03):361373, 2004. doi: 10.1051/epjap:2004165.

Donald W. McRobbie, Elizabeth A. Moore, Martin J. Graves, and Martin R. Prince. MRI from Picture to Proton. Cambridge University Press, September 2006. ISBN 9781139457194.

Joseph D. Seymour, Bertram Manz, and Paul T. Callaghan. Pulsed gradient spin echo nuclear magnetic resonance measurements of hydrodynamic instabilities with coherent structure: Taylor vortices. Physics of Fluids, 11(5):1104-1113, May 1999. 
ISSN 10706631. doi: doi:10.1063/1.869981. URL http://pof.aip.org/resource/ 1/phfle6/v11/i5/p1104_s1?isAuthorized=no.

A. J Shaka, S. P Rucker, and A Pines. Iterative carr-purcell trains. Journal of Magnetic Resonance (1969), 77(3):606-611, May 1988. ISSN 0022-2364. doi: 10.1016/ 0022-2364(88)90022-4. URL http://www.sciencedirect.com/science/article/ pii/0022236488900224.

G. Shiko, A.J. Sederman, and L.F. Gladden. MRI technique for the snapshot imaging of quantitative velocity maps using RARE. Journal of Magnetic Resonance, 216:183191, March 2012. ISSN 1090-7807. doi: 10.1016/j.jmr.2012.01.021. URL http://www. sciencedirect.com/science/article/pii/S1090780712000560. 



\section{Rheo-NMR and Rheo-USV of $10 \%$ $\mathrm{CPCl}$}

The goal in this chapter is to characterize the steady state shear flow of a specific wormlike micellar system that has been subject to numerous studies in the past 20 years. To this end, the methodology developed in the previous chapter shall be applied to measure the flow of the WLM solution in the cylindrical Couette cell (B) with good accuracy. Particular emphasis is on the temporal and spatial resolution needed to correctly describe the dynamics of the shear banded state with varying applied shear rate. Apart from a mere transition to a shear banded state a series of other flow instabilities have been observed in recent years for this particular system. These include axial structures in the flow field, generally known as 'vorticity undulations', the presence of unsteady slip conditions and fluctuative behaviour such as turbulent bursts potentially caused by elastic instabilities. All of these instabilities are described in more detail during the course of this chapter. With regards to the NMR method, it shall be assessed whether these structures can be resolved given the capabilities in terms of spatial and temporal resolution. In order to make the study more complete and to provide a means of comparison for the NMR technique, results are compared to those of a recently developed 2D ultrasound velocimetry method (Gallot et al. (2013)), which offers higher temporal resolution. Furthermore, two different surface characteristics for the inner cylinder of the Couette cell are used for the ultrasound method in order to highlight the sensitivity of the flow dynamics to the imposed boundary conditions.

The structure of the chapter is as follows. First the sample is introduced and the bulk rheology characterized. After a short survey on the experimental parameters for the NMR method, basic principles and the experimental setup of the ultrasound method are outlined. Results start off with spatially and temporally averaged velocity profiles as obtained for two different (read) resolutions in the NMR case and for two different surface characteristics for the USV method. Then flow structures in the axial direction are 
discussed and evaluated. Finally, the fluctuative nature of the steady state flow is studied with particular focus on the emergence of elastic instabilities and turbulent bursts.

\subsection{The Sample}

The sample under study is made of $10 \%(\mathrm{w} / \mathrm{w})$ cetylpyridinium chloride $(\mathrm{CPCl})$ and sodium salicylate (NaSal) with a molar ratio of 2:1 diluted in $0.5 \mathrm{M} \mathrm{NaCl}$-brine. It shall be referred to as ' $10 \% \mathrm{CPCl}$ ' here. The presence of both $\mathrm{Sal}^{-}$and $\mathrm{Cl}^{-}$ions causes a screening of the electrostatic interaction of the (positive) $\mathrm{CP}^{+}$head group which leads to the formation of long wormlike micelles already at the critical micellar concentration (Berret (2006)). The concentration regime for the present solution lies in the semi-dilute range where entangled micelles form an elastic network and thus make the solution viscoelastic. All experiments have been performed at a controlled temperature of $25^{\circ} \mathrm{C}$. This specific solution and other closely related wormlike micellar systems have been the subject of a large body of studies in recent decades. In the following section, a selection of these studies that are regarded particularly important for the present work shall be highlighted. Afterwards, the preparation procedure and the general bulk rheology of the $10 \% \mathrm{CPCl}$ system are discussed.

\subsubsection{Previous Studies}

The first exhaustive rheological studies of $\mathrm{CPCl} / \mathrm{NaSal}$ wormlike micellar systems in the non-linear regime were done by Berret et al. (1994). The appearance of a stress plateau in the flow curve was related to a first-order phase transition from an isotropic entangled to more ordered nematic state in parts of the fluid induced by the shear flow. Due to the alignment of the micelles in the flow direction, the viscosity of the nematic phase is expected to be much lower than that of the isotropic state. Consequently, it was argued that an increase of the applied shear rate in the range of the stress plateau leads to a change of relative proportions between the isotropic and nematic phase in a way such that a constant stress is maintained. These considerations are basically the idea behind the simple lever rule introduced in section 3.4. However, this study was based on bulk rheology and this did not offer spatially resolved flow information. The first direct detection of shear banding was by Britton and Callaghan (1997), who used magnetic resonance velocimetry to measure the shear flow of a $\mathrm{CPCl} / \mathrm{NaSal}$ solution confined to a cone-and-plate geometry. Multiple regions with distinctively different velocity gradients were found along the fluid gap. Salmon et al. (2003) found that a $6 \% \mathrm{CPCl}$ system at a temperature of $21.5^{\circ} \mathrm{C}$ sheared 
in a Couette geometry followed the simple lever rule as depicted in Fig. 3.11b using a light scattering technique to measure fluid velocities. The high shear rate band was nucleating at the inner wall where the relative local stress is highest over the gap. This structure of exactly two shear bands in the case of cylindrical Couette shear flow has been confirmed by many studies thereafter. Investigations by López-González et al. (2004) focusing on the $10 \% \mathrm{CPCl}$ system had three major findings. Firstly, the existence of a more ordered fluid phase in the shear banded state was confirmed by means of spatially resolved NMR spectroscopy. Secondly, it was found that the proportion of this nematic phase increases with the applied shear rate, as expected from the simple lever rule. However, as the third main result, it was observed by means of MRV that the shear banded structure shows fluctuations over a measurement period of $60 \mathrm{~s}$, which exceeded the temporal resolution of the spectroscopy method, which was in the order of hours. Thus, the necessity of sufficient temporal resolution for a complete description of the banded flow dynamics became obvious. Another flow instability was pointed out by Lerouge et al. (2008). For a CTAB based WLM solution undulation patterns in the axial profile of the interface between the low and high shear rate bands were reported. This underlined the importance of a $2 \mathrm{D}$ velocimetry technique that not only resolves velocities in the radial but also in the axial direction.

In a more recent publication, Feindel and Callaghan (2010) reported on a so-called 'inversed lever rule' behaviour for the $10 \% \mathrm{CPCl}$ system at a temperature $25^{\circ} \mathrm{C}$. In contrast to the simple lever rule, the authors observed a constant interface position and changing shear rates in the bands over the range of applied shear rates within the stress plateau for time averaged velocity profiles obtained with a PGSE-RARE sequence. Furthermore, a considerable amount of slip at the inner rotating gap wall was noticed. A variable shear rate in the low viscosity band and wall slip had been noted previously for similar systems (Helgeson et al. (2009); Raudsepp and Callaghan (2008)), however, always in conjunction with a constant value for the shear rate in the low shear rate band and a variable proportion of the high shear rate band; a behaviour referred to as 'standard anomalous lever rule' behaviour by Fardin et al. (2012a). Feindel and Callaghan reasoned that their unexpected finding was induced by a destabilization of the banded flow state due to fluctuating slip conditions at the rotor wall in combination with the application of a small shear rate step protocol. Instead of applying a shear rate quench, where the desired shear rate is imposed directly on the system originally at rest, $\dot{\gamma}_{a}$ was incremented in small steps in order to avoid stress overshoots. Additionally, flow structures in the vorticity direction in the order of centimeters were found. Furthermore, it shall be noted that a single slice with a thickness of $4 \mathrm{~mm}$ and a linear $k$-space scheme were used for the PGSE-RARE sequence. 
The dimensions of the Couette cell were nearly identical to those of cell A (see section 5.1) and thus slightly bigger than those of cell $\mathrm{B}$, which is used here.

In contrast to Feindel and Callaghan (2010), an even more recent study by Fardin et al. (2012a) on the same system at a lower temperature of $21.5^{\circ} \mathrm{C}$, using flow turbidity and 1D Rheo-USV experiments, observed the standard anomalous lever rule behaviour mentioned earlier. Apart from fluctuating slip conditions, the emergence of secondary flows caused by elastic instabilities were identified as the main origin of this behaviour. Furthermore, it was found that UV light exposure considerably altered the flow behaviour of the sample by increasing the overall amount of slip present. The idea was put forward that for an irradiated sample the increased slip could lead to an inversed lever rule observation if the range of studied shear rates is restricted to a small part of the stress plateau. The discrepancy in the results of the latter two studies shall be addressed in this chapter.

Finally it shall not remain unnoted, that there has also been a tremendous amount of theoretical work published on the rheology of wormlike micelles in recent years. However, a survey of these studies is beyond the scope of this thesis. The review article by Cates and Fielding (2006) provides an overview over findings, whereas a more recent study can be found in Moorcroft and Fielding (2013), for example.

\subsubsection{Sample Preparation and Rheology}

For the preparation of the NMR samples, the desired quantities of the $\mathrm{CPCl}$ and NaSal salt were separately weighted and dissolved in the brine solution. After a period of about an hour, during which both solutions were occasionally shacked, the two solutions were mixed in an amber glass jar and stored at $30^{\circ} \mathrm{C}$ in an oven for several days before experiments were performed. The latter ensured that the wormlike micelles had fully developed and the solution was in an equilibrium state. Additionally, $0.15 \mathrm{mM} \mathrm{GdCl}_{3}$ were added to act as a relaxation agent resulting in relaxation times $T_{2} \approx 300 \mathrm{~ms}$ and $T_{1} \approx 500 \mathrm{~ms}$ for the WLM solution. At this point it should be stressed that the velocimetry sequence essentially measures the velocity of the solvent water, whereas the ${ }^{1} \mathrm{H}$ protons in the surfactant only give a negligible contribution to the NMR signal. This is not regarded to be a complication in the present case however, as a distinction between solvent and micelle flow is regarded as being artificial.

In Feindel and Callaghan (2010), it was shown that the addition of $0.15 \mathrm{mM} \mathrm{GdCl}_{3}$ did not have an effect on the bulk rheology of the sample. The flow curve depicted in Fig. 6.1 was recorded using a TA-2000 rheometer equipped with a steel cone-and-plate geometry with $60 \mathrm{~mm}$ diameter and a $2^{\circ}$ angle. All stress values were acquired in a single shear rate 
sweep averaged over the last $30 \mathrm{~s}$ of each $60 \mathrm{~s}$ long step. The flow curve exhibits a plateau region starting at $\dot{\gamma}_{a} \approx 3 \mathrm{~s}^{-1}$ and matches previous observations by Feindel and Callaghan (2010). At shear rates higher than $25 \mathrm{~s}^{-1}$, which are not considered in the present study, the flow gets very unstable and the sample spurts out of the cone-and-plate geometry.

The ultrasound measurements were performed in the group of Sébastien Manneville at the ENS in Lyon. The $10 \% \mathrm{CPCl}$ sample was prepared and treated similarly to the NMR case, however no $\mathrm{GdCl}_{3}$ was added. Instead tiny glass beads were seeded into the solution required for the ultrasonic velocimetry measurements, as shall be described in section 6.2.2. The question whether or not these small seeding particles change the overall flow dynamics of the sample has not been raised explicitly. However, this possibility could serve as an explanation for some of the differences between NMR and ultrasound results as shall be seen later. Nonetheless, the flow curve for the ultrasound sample was essentially in agreement with the one shown for the NMR sample (Fig. 6.1).

Great care was taken to minimize light exposure of all samples to exclude photo degradation effects as discussed in Fardin et al. (2012a). To this end amber bottles were used for the storage and the sample has covered with aluminium foil whenever outside of the NMR magnet. Consequently, photo degradation is not considered to play a role in the current study.

\subsection{Experimental}

\subsubsection{Rheo-NMR}

In chapter 5 the methodology for the PGSE-RARE sequence (Fig. 5.3) was been developed with great care. This sequence shall now be used to characterize the shear flow of the $10 \%$ $\mathrm{CPCl}$ system. To this end, the conventional 2D method as described in section 5.2.1 and the 1D high resolution variant (see section 5.3.3.1) is used. The former and latter shall be referred to as 'NMR LR' (LR: low resolution) and 'NMR HR' (HR: high resolution), respectively, in accordance with the resolution achieved in the read direction. In both cases the parameters for the PGSE part $(\delta=1 \mathrm{~ms}, \Delta=20 \mathrm{~ms})$, as well as the orientation for the read $(x)$, phase $(z)$ and slice $(y)$ directions were chosen as in section 5.2.1. The nonzero velocity encoding gradient $g_{2}$ was set to values between $0.02 \mathrm{~T} \mathrm{~m}^{-1}$ and $0.25 \mathrm{~T} \mathrm{~m}^{-1}$ depending on the applied shear rate in order to maximize the flow induced phase spread over the Argand plane. Furthermore, the inter-excitation repetition time $T_{\text {scan, } \mathrm{R}}$ was $0.5 \mathrm{~s}$ which resulted in a temporal resolution $T_{\mathrm{R}}$ of $2 \mathrm{~s}$ as the two step phase cycle discussed in section 5.2.4 was applied for both NMR LR and HR. Following the finding in sec- 


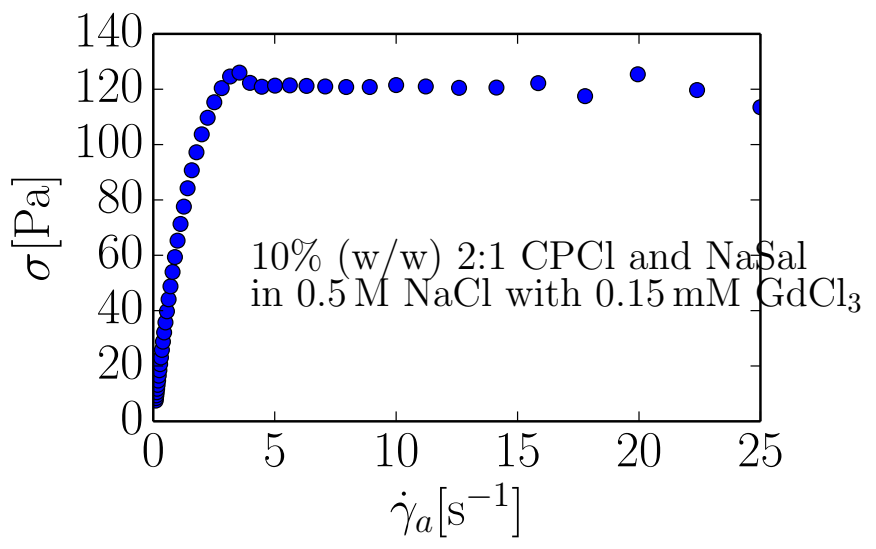

Fig. 6.1: Bulk rheology of the wormlike micellar solution under study. A steel cone-andplate device with $60 \mathrm{~mm}$ diameter and $2^{\circ}$ angle has been used to record the flow curve. Stress values were taken in a single sweep (low to high) and are an average over the last $30 \mathrm{~s}$ of each $60 \mathrm{~s}$ step.

tion 5.2.2.1, the thickness of the PGSE slice was set to $1 \mathrm{~mm}$. Due to the importance of stable thermal conditions for the sheared sample, NMR experiments were performed with Couette cell B, which allowed for active temperature control with the Bruker system. The fill height $H$ of the fluid was about $12 \mathrm{~cm}$ measured from the bottom of the cell and thus extended considerably beyond the active measurement region at the top (see Fig. 5.2b). Both the NMR LR and HR experiments were performed with the same sample load over a period of two days over which the cell was not taken out of the magnet. As no marker fluid was present, the gap calibration was done using the magnitude method (see section 5.2.5). It shall also be mentioned that the PEEK rotor wall was slightly sandblasted.

With particular regard to NMR LR, a grid of $256 \times 16 k$-space points was acquired with the FOV set to $20 \mathrm{~mm} \times 25 \mathrm{~mm}$ in the read-phase $(x-z)$ imaging plane resulting in a resolution of about $80 \mu \mathrm{m} \times 1.56 \mathrm{~mm}$. The CPMG train itself consisted of 32 echoes where each line in $\mathbf{k}$-space was traversed twice, first for the odd and then for the subsequent even echo. However, only the even echoes were retained for image construction resulting in 16 points in the phase direction. Furthermore, the centric scheme was applied. Concerning NMR HR, the resolution in the read direction was $16 \mu \mathrm{m}$ for a FOV of $2 \mathrm{~mm}$ corresponding to about 64 points over the $1 \mathrm{~mm}$ gap extent as compared to approximately 13 points for NMR LR. The thickness of the RARE slice was set to $25 \mathrm{~mm}$ in both cases.

The procedure for a single experiment was as follows. A shear rate $\dot{\gamma}_{a}$ was imposed on the sample synchronously with the start of the NMR acquisition. After a series of 450 flow maps had been acquired over a period of about $900 \mathrm{~s}$, shear was stopped and the sample 
was at rest for about $300 \mathrm{~s}$ before the next experiment was started. This way of imposing the shear on the sample corresponds to the shear quench scheme described earlier.

\subsubsection{Rheo-USV}

For the sake of a more complete picture on the flow dynamics of the $10 \% \mathrm{CPCl}$ system and in order to provide a means of comparison for the NMR measurements, a second velocimetry technique has been applied for the study. This recently developed Rheo-USV (Ultra-sonic Speckle Velocimetry) technique is capable of acquiring 2D velocity maps of fluids sheared in a cylindrical Couette geometry with high spatial and temporal resolution (Gallot et al. (2013)). In particular, the high resolution in the vorticity direction as well as the very high frame rates achieved in the acquisition of the velocity maps allow for a better understanding of the shear flow and provide a guide for the interpretation of the NMR data. In the following, key principles of the technique shall be outlined briefly along with a description of the experimental setup and the parameters used for the acquisition. However, a more in-depth introduction is beyond the scope of the thesis. The interested reader shall be referred to Gallot et al. (2013) for a detailed explanation on the Rheo-USV technique.

The 2D Rheo-USV method is an extension of the 1D USV technique developed by Manneville et al. (2004). As shown in Fig. 6.2a, the setup consists of an ultrasonic scanner that is coupled to a commercial rheometer (TA Instruments ARG2), which is used to impose the shear motion on the fluid. The ability to record the torque exerted by the fluid on the rotor allows for a simultaneous measurement of the flow velocity and rheological parameters, such as the shear stress. Restrictions on the dimensions of the cylindrical Couette geometry are only set by the capabilities of the rheometer itself, e.g. the maximum torque the rheometer can apply, in contrast to the NMR technique where the shear cell has to fit into the NMR magnet. In the present study, the radius of the inner rotating cylinder was chosen to be $23 \mathrm{~mm}$ whereas the stator had a radius of $25 \mathrm{~mm}$ resulting in a gap size of $2 \mathrm{~mm}$. For the PEEK rotor two different surface characteristics were available, namely smooth (polished) and rough (sandblasted). The outer wall was made out of glass which allows for optical surveillance of the sample while in the geometry. A plug described in Lerouge et al. (2008) is used to prevent the formation of bubbles at the top of cell, i.e. at the fluid air interface. The height of the cell was about $50 \mathrm{~mm}$. The water tank enclosing the geometry has two functions. Firstly, it allows for precise control of the sample temperature via the connection to an external water bath. Secondly it ensures a 
constant sound speed along the acoustic path of the ultrasound beam, which is important for the velocity measurement.

The ultrasound technique itself relies on the acquisition of ultrasonic speckle signals formed by interferences of the backscattered echoes from incident pulses. The recorded signal is partitioned into equally sized time intervals where each interval corresponds to a certain time-of-flight of the ultrasound pulse. As the speed of sound is nearly constant within the apparatus, each time-of-flight can be directly mapped onto a spatial position along the acoustic path (see Fig. 6.2b). To allow for the detection of motion this procedure is applied to a series of ultrasound pulses which are emitted periodically. Cross correlating the recorded signal of successive pulses for each time interval reveals temporal shifts in the speckle pattern which are linked to the motion of the scatterer. The time shift can be directly transformed into a spatial displacement along the acoustic path using the known speed of sound. To get the corresponding velocity, one divides the displacement length by the time delay between two successive ultrasound pulses. Finally, this beam path velocity $v_{\text {beam }}(r)$ (beam path is shown Fig. $6.2 \mathrm{~b}$ ) has to be transformed into the velocity component of interest, the tangential velocity $v_{\varphi}(r)$. As thoroughly described in Manneville et al. (2004), one assumes that the flow is purely tangential and uses basic trigonometry combined with a small gap approximation to infer $v_{\varphi}(r)$ from $v_{\text {beam }}(r)$. As the assumption of a purely tangential flow may not generally hold for the flow discussed in this chapter, it shall be mentioned that $v_{\text {beam }}(r)$ can contain a significant contribution from the radial velocity component (see Gallot et al. (2013))

$$
v_{\text {beam }}(r) \simeq v_{\varphi}(r)+\frac{v_{r}(r)}{\tan (\phi)} .
$$

Furthermore, it shall be noted that the incident angle $\phi$, which was about $7^{\circ}$ here, defines the width of the active measurement region, similar to the (PGSE) slice thickness in the NMR case (see Fig. 6.2b). For the current setup this width is about $300 \mu \mathrm{m}$ and therefore curvature effects as discussed in section 5.2.2.1 are not regarded to be important.

The acoustic contrast is either given by particles already present in the solution, such as colloids, or if needed by seeding the solution with an acoustic contrast agent. For the present system, the latter was necessary and a dilute aqueous suspension of polydisperse hollow glass spheres (Potters Spherical, mean diameter $6 \mu \mathrm{m}$, mean density $1.1 \mathrm{~g} / \mathrm{cm}^{3}$ ) was added. To resolve structures in the vorticity direction 128 ultrasonic transducers are stacked over a length of $32 \mathrm{~mm}$ forming the ultrasonic array depicted in Fig. 6.2a. The array is approximately centred in the axial direction relative to the Couette cell. Each transducer is $200 \mu \mathrm{m}$ wide and spaced by $50 \mu \mathrm{m}$ resulting in fixed resolution in the vorticity 
direction of $250 \mu \mathrm{m}$. The pixel size in the gradient direction is given by the width of the time intervals used for the cross correlation. A value of $65 \mu \mathrm{m}$ provided good resolution over the gap while ensuring a wide enough time window for the cross correlation algorithm.

In the present study two different acquisition schemes have been used. For the first, referred to as 'USV TL' (TL: Temporal Low), a flow map has been generated every second over a period of $250 \mathrm{~s}$ starting with shear inception. Each single velocity map is an average over 50 pulses, that were emitted periodically over $50 \mathrm{~ms}-200 \mathrm{~ms}$ depending on the applied shear rate. For the second, referred to as 'USV TH' (TH: Temporal High), 8000 pulses were applied without interruption a couple of minutes after shear startup using the same shear rate depended pulse frequency as for USV TL. A single velocity map is again composed of 50 pulses. To increase the nominal temporal resolution, however, the averaging window is only shifted by 25 pulses for consecutive time steps resulting in a repetition time of $25 \mathrm{~ms}-100 \mathrm{~ms}$ with total number of 320 velocity maps acquired. Note, that the first acquisition scheme (USV TL) has a repetition time (1s) similar to the NMR experiment $(2 \mathrm{~s})$ and therefore is primarily used where the two experimental techniques are compared. If not stated otherwise, USV results discussed in this chapter were obtained using USV TL.

(a)

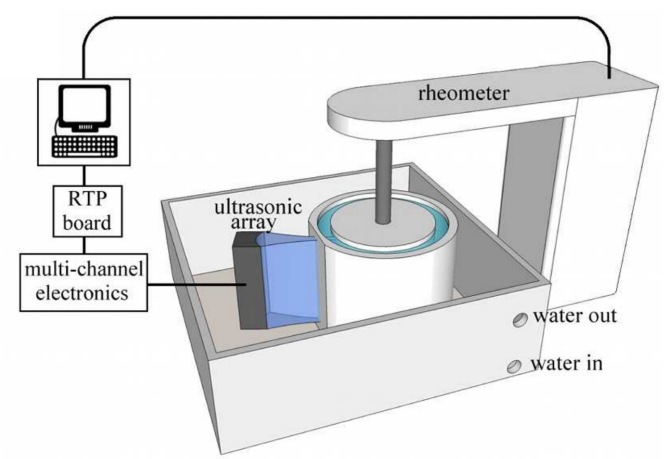

(b)

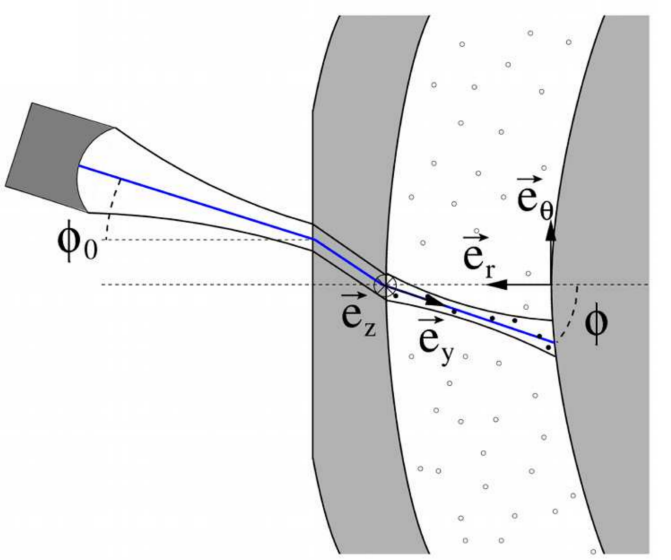

Fig. 6.2: (a) General setup of the USV apparatus. (b) Top view on the gap of the USV Couette cell together with the acoustic beam path (blue line). The incident angle $\phi$ ensures sensitivity for tangential velocity, as only displacements along the beam path can be measured. Furthermore, $\phi$ defines a slice thickness similar to the NMR experiments. In the setup used herein, this slice thickness is about $300 \mu \mathrm{m}$. Adapted from Gallot et al. (2013).

Finally it shall be outlined what makes this method particularly interesting with regards to the NMR technique. First of all, the experimental outcome, a 2D velocity map, is similar 
to the 2D NMR velocimetry experiment and allows a direct comparison of the flow maps if differences in resolution (spatial and temporal) are taken into account. However, the two methods are based on completely different physical principles. Agreement of experimental results from both techniques would therefore be a strong hint towards a correct description of the flow dynamics by the methods in question. Still, differences in the particular setup, such as the dimensions of the Couette geometry have to be considered. A strength of the USV method is the high temporal resolution available. It is possible to capture up to 10, 000 velocity maps per second, depending on the flow speed (Gallot et al. (2013)). In order to obtain flow maps with a good SNR, one averages over a certain number of single flow maps. Consequently a realistic temporal resolution for our setup is in the range of $25 \mathrm{~ms}-100 \mathrm{~ms}$ as used for the USV TH acquisition scheme described earlier. This high temporal resolution allows for a better understanding of steady flow fluctuations and makes it possible to resolve sub-second transient flow phenomena such as turbulent bursts described in section 6.3.2.2. To this end, it also gives insight into the flow processes during the acquisition of a single 2D NMR velocity map over a time span of $2 \mathrm{~s}$. However, as discussed in more detail later, the time over which velocity information is accumulated $(\approx 50 \mathrm{~ms})$ is similar for NMR and USV experiments. In Table 6.1 a comparison of spatial and temporal resolution for the NMR and USV methods used in this study is given.

\begin{tabular}{ccccc}
\hline & NMR LR & NMR HR & USV TL & USV TH \\
\hline \hline Spatial resolution: & & & & \\
Gradient direction & $80 \mu \mathrm{m}$ & $16 \mu \mathrm{m}$ & $65 \mu \mathrm{m}$ & $65 \mu \mathrm{m}$ \\
Vorticity direction & $1.56 \mathrm{~mm}$ & $-(1 \mathrm{D})$ & $0.25 \mathrm{~mm}$ & $0.25 \mathrm{~mm}$ \\
Temporal Resolution: & $2 \mathrm{~s}$ & $2 \mathrm{~s}$ & $1 \mathrm{~s}$ & $25 \mathrm{~ms}-250 \mathrm{~ms}$ \\
& & & & $\left(\right.$ depending on $\left.\dot{\gamma}_{a}\right)$ \\
Approx. gap points: & 13 & 64 & 30 & 30
\end{tabular}

Table 6.1: Comparison of spatial and temporal resolution for the techniques and methods applied. The temporal resolution refers to the repetition time $T_{R}$ for successive flow maps. 'Approx. gap point' stands for the approximate number of data points over the Couette gap and thus is an indicator for the effective resolution of the method.

\subsection{Results}

Time-resolved velocity maps for both NMR and USV methods provide a wealth of information on the flow dynamics of the $10 \% \mathrm{CPCl}$ system in the range of applied shear rates from $2 \mathrm{~s}^{-1}$ to $24 \mathrm{~s}^{-1}$, which are investigated for this study. In order to unravel this 
information, the focus shall first be on spatially and temporally averaged velocity profiles, where good agreement for both techniques is found and the fluid roughly follows a standard anomalous lever rule as previously observed by Fardin et al. (2012a). Secondly, dynamics and structures in the vorticity direction are presented. These regular undulations are only detected for the USV method at specific applied shear rates. Thirdly, a closer look is taken on the nature of turbulent bursts that occur for imposed shear rates that lie towards the end of the investigated $\dot{\gamma}_{a}$-range. In particular, bursts are apparent in the USV results, whereas the NMR measurements do not show a direct sign of them for equivalent shear rates. Finally the flow is discussed from a statistical point of view, where broad distributions are found for the main flow characteristics obtained by analyzing non-averaged velocity profiles individually.

\subsubsection{Averaged Velocity Profiles}

Averaged velocity profiles give a general impression on the dynamics of the fluid and shall be discussed for both techniques in the following. For better quantification and comparison, velocity profiles are fitted with a piece-wise linear function assuming two bands with the rotor wall at the origin. The relevant parameters extracted are the slopes in the low and high shear rate band $\left(\dot{\gamma}_{l}, \dot{\gamma}_{h}\right)$ and the proportion of the latter $\alpha_{h}$. Using these regression values, one can calculate the relative slip $v_{s} / V$, with the slip velocity $v_{s}$ defined as the difference between the rotor velocity $V$ and the fluid velocity at the rotor $v(0)$. Pixels situated in the transition region between the high and low shear rate bands have not been included in the fit as well as points for NMR HR where velocity determination was not possible due to perturbations from external rf-signals.

NMR A qualitative comparison of NMR HR and NMR LR is given in Fig. 6.3 and Fig. 6.4 where time- and space-averaged (along $z$ for NMR LR) velocity profiles are shown for applied shear rates in the range from $2 \mathrm{~s}^{-1}$ to $12 \mathrm{~s}^{-1}$ and $14 \mathrm{~s}^{-1}$ to $24 \mathrm{~s}^{-1}$, respectively. The average is taken over 400 individual flow maps recorded over a period of $800 \mathrm{~s}$ after the transient startup behaviour. Below the shear banding transition at $\dot{\gamma}_{a}=2 \mathrm{~s}^{-1}$ the profiles are linear and exhibit a slight amount of slip for both methods. Generally, slip can be inferred from the mismatch of the fluid velocity at the inner wall $v(0)$ and the velocity of the inner wall $V \approx \dot{\gamma}_{a} d$, with $d$ being the respective gap size ( $1 \mathrm{~mm}$ in the NMR case). The observation of slip below the shear banding transition is not expected. However, slip has also been observed for a Newtonian fluid at this shear rate (Fig. 5.20a) for NMR HR and thus might be an artefact from experimental imperfections. For imposed shear rates 
above the shear banding threshold $\left(\approx 3 \mathrm{~s}^{-1}\right)$, it is obvious that the flow separates into two bands with differing velocity gradients. As expected, the high shear rate portion of the fluid is nucleating at the rotor wall and growing with increased applied shear rate. Judging from the fitted velocity profiles there is generally more slip for NMR HR as compared to NMR LR at identical applied shear rates (except for $\dot{\gamma}_{a}=6 \mathrm{~s}^{-1}$ ). This might be caused by uncertainties in the calibration process for NMR LR and shall be discussed later. Slip at the outer wall is not observed within the precision of the technique. Furthermore, the interface between the two bands gets less sharply defined for higher shear rates indicating the existence of flow instabilities. In the case of NMR LR, errorbars show the standard deviation for each of the data points. It can be observed that these deviations are larger in the high shear rate band suggesting that the flow of the low shear rate band is more stable. In the case of NMR HR, errorbars are not shown due to the low SNR of the underlying data.

For NMR LR only one pixel is fully contained in the high shear rate band at applied shear rates $\dot{\gamma}_{a}<15 \mathrm{~s}^{-1}$ making a precise quantification of the slope impossible. Therefore the values for $\dot{\gamma}_{h}$ and $\alpha_{h}$ as obtained from the linear regression in that shear rate range are not necessarily meaningful and the slip value calculated from the extrapolation of the velocity profile towards the rotor wall is somewhat speculative. In contrast, NMR HR clearly shows the existence of a low viscosity band for imposed rates as low as $4 \mathrm{~s}^{-1}$ and captures its expansion and growing steepness for increasing $\dot{\gamma}_{a}$. The higher number of velocity points in the region of high shear also make a more precise detection of slip possible.

To allow for a more quantitative analysis the main parameters of the linear regression are plotted as a function of the imposed shear rate in Fig. 6.5. Firstly, an only moderately increasing slope in the low shear rate band $\dot{\gamma}_{l}$ is observed (Fig. 6.5a) for both NMR methods. As this band extends over the length of multiple pixels, agreement for both resolutions is expected. Indeed, apart from a small offset, HR and LR are well within error ranges. The nearly constant shear rate fits into the classical shear banding picture where it marks the beginning of the stress plateau. It shall be emphasized that active temperature control ensured constant thermal conditions for the system under shear. The lack of the former can result in heating of the sample by the imaging system during the experiment. As the viscosity of the WLM solution is sensitive to small temperature changes (Lerouge and Berret (2010)), an increase of the velocity gradient in the low shear rate band over the course of a potentially long experiment would not be unexpected, if no active temperature control was in place. In Feindel and Callaghan (2010) a small step protocol was employed, where the fluid was continuously imaged while the applied 

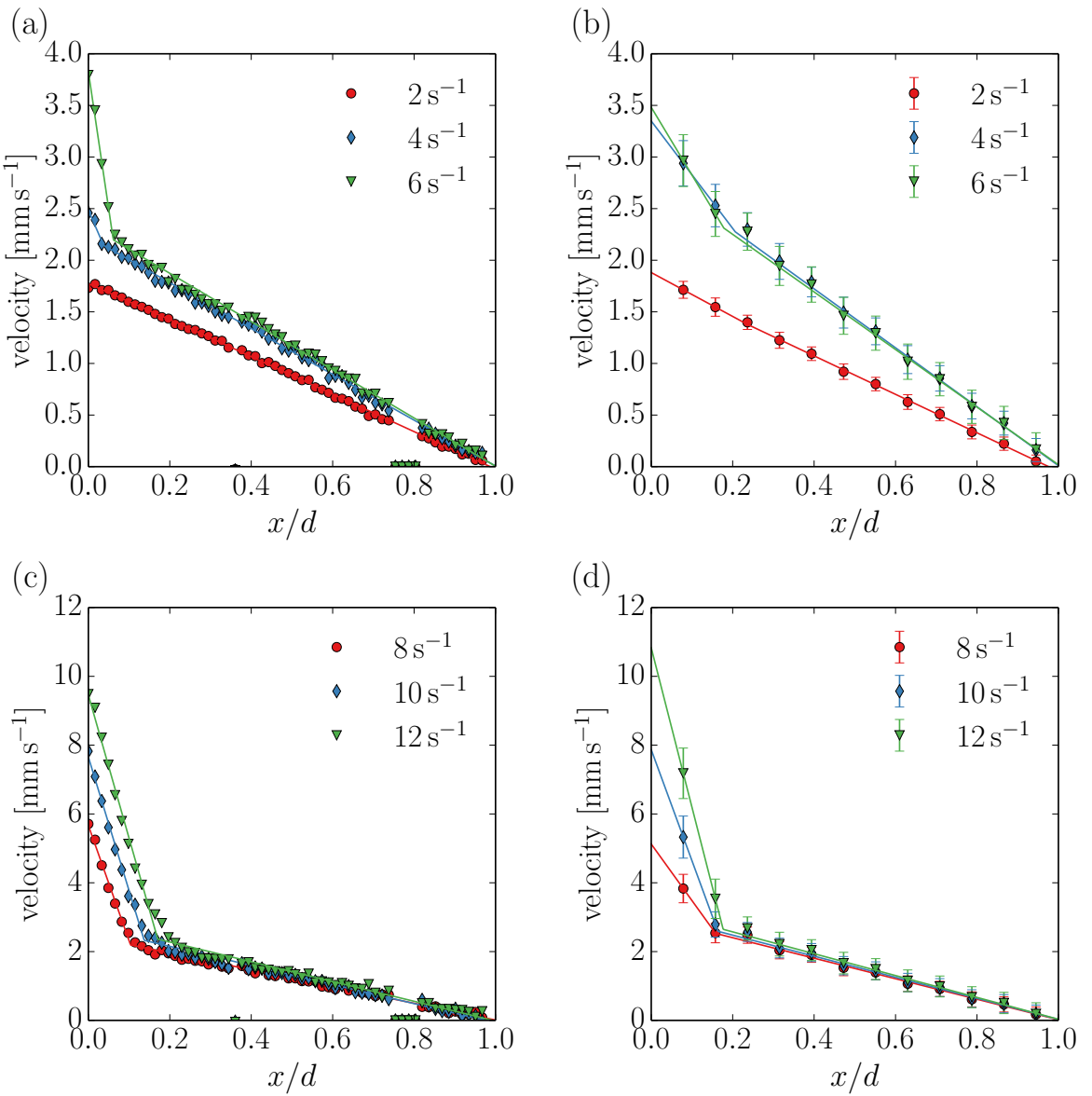

Fig. 6.3: Spatially and temporally averaged velocity profiles for NMR HR (a,c) and NMR LR $(b, d)$ at various applied shear rates. The averages are taken over $800 \mathrm{~s}$ after the transient startup behaviour with a repetition time of $2 \mathrm{~s}$ for each single flow map. Symbols represent the velocity averages of each voxel and the solid lines are piece-wise fits assuming two bands. Errorbars showing the standard deviation for each single data point have been omitted in the case of NMR HR due to their large extent caused by the low SNR of the method.

shear rate was ramped in small steps. Hence, the sample was not allowed to cool between successive values of $\dot{\gamma}_{a}$. Furthermore, no active temperature control was used (Feindel (2013)). These circumstances might have led to an increase in $\dot{\gamma}_{l}$ from $2 \mathrm{~s}^{-1}$ to $5 \mathrm{~s}^{-1}$ over the range of applied shear rates (up to $15 \mathrm{~s}^{-1}$ ), which is not found for the present study on the same system.

Secondly, the shear rate in the low viscosity band (Fig. 6.5b) is characterized by a rapid increase to a value of about $40 \mathrm{~s}^{-1}$ at $\dot{\gamma}_{a}=10 \mathrm{~s}^{-1}$ for NMR HR. As NMR LR is not delivering reliable values in that range of imposed shear rates, huge difference are found 
(a)

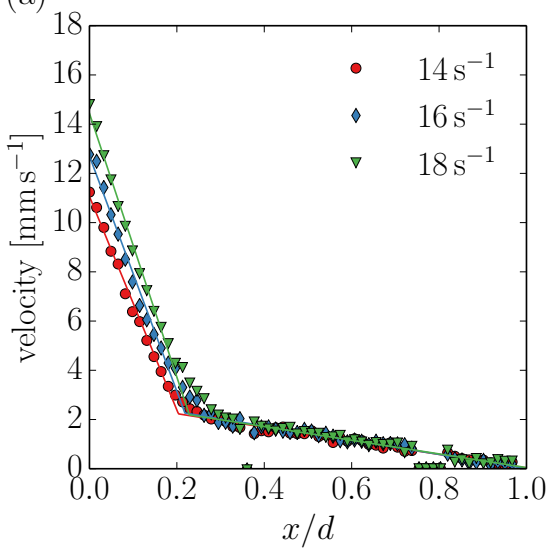

(c)

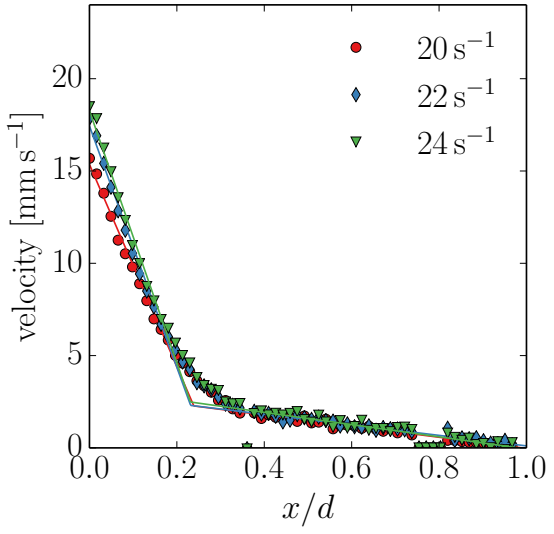

(b)

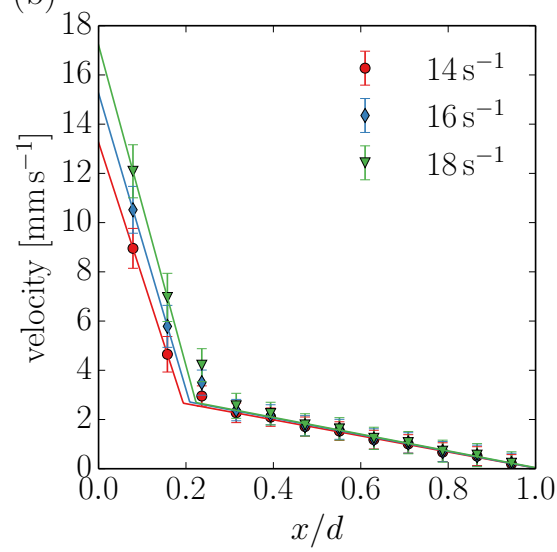

(d)

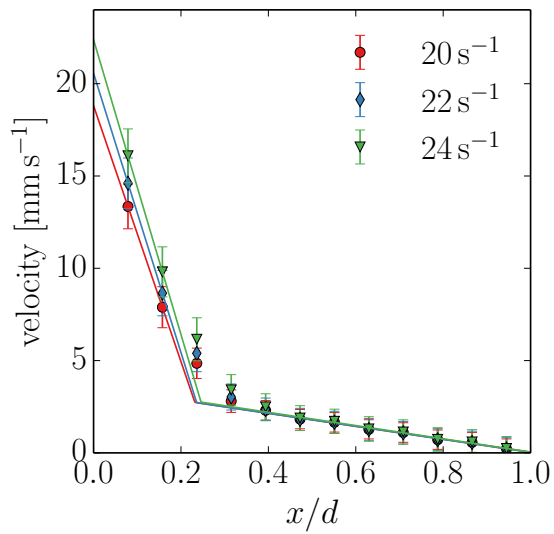

Fig. 6.4: Same as Fig. 6.3, but for applied shear rates in the range of $14 \mathrm{~s}^{-1}$ to $24 \mathrm{~s}^{-1}$.

here. For higher $\dot{\gamma}_{a}$ an offset of about $10 \mathrm{~s}^{-1}$ is observed, whereas the rate of change is similar for both methods. The offset might be caused by uncertainties in the gap calibration for NMR LR.

Thirdly, NMR LR cannot pickup the migration of the interface position as captured in the high resolution case, (Fig. 6.5c). In the latter a linear increase up to a shear rate of $15 \mathrm{~s}^{-1}$ is observable. For higher applied shear rates, $\alpha_{h}$ plateaus indicating a change in the flow behaviour. This feature might have been caused by flow instabilities as shall be discussed later. NMR LR delivers results very similar to those of NMR HR for $\dot{\gamma}_{a} \geq 15 \mathrm{~s}^{-1}$. However, the trend suggested by NMR LR is slightly increasing and not fully hinting towards a plateau. Still, differences in this shear rate range are small and might be caused by experimental imperfections.

Fourthly, the relative slip characteristics (Fig. 6.5d) are similar for both methods with a maximum around $\dot{\gamma}_{a} \approx 5 \mathrm{~s}^{-1}$ followed by a decrease towards an asymptotic value of 
0.2 for NMR HR and 0.05 for NMR LR. Although the qualitative behaviour for both methods is similar, the discrepancy for higher shear rate values is apparent. It shall be noted however, that there is a wide range of possible slip velocities for the LR case if one assumes an uncertainty of half a pixel width in determining the exact inner wall position as indicated by the errorbars in Fig. 6.5d. Hence, the differences in the asymptotic values might be due to this uncertainty in the calibration. Furthermore, the amount of slip is generally lower as compared to previous results by Feindel and Callaghan (2010), possibly due to the refined experimental parameters used in this study (PGSE slice thickness, $k$-space scheme) and the different specificities of the Couette geometries in use.

It can be concluded that NMR LR and HR results show relatively good agreement for shear rates above $15 \mathrm{~s}^{-1}$. The remaining deviations observed in this $\dot{\gamma}_{a}$-range might be caused by experimental imperfections and errors in the calibrations procedure.

USV The corresponding averaged profiles for USV are shown in Fig. 6.6 for smooth $(\mathrm{a}, \mathrm{c})$ and rough $(\mathrm{b}, \mathrm{d})$ wall characteristics. For most of the profiles the average is taken over 100 $2 \mathrm{D}$ velocity maps that were recorded $150 \mathrm{~s}$ after the inception of shear over a period of 100 s. At higher shear rates the occurrence of turbulent bursts starts dominating the flow behaviour after a certain time $t_{b}$ in the range from $30 \mathrm{~s}$ to $100 \mathrm{~s}$ following shear startup. Therefore the averaging window is restricted to a period $\left[t_{a}, t_{b}\right]$, where $t_{a}$ marks the end of the transient behaviour following shear inception. $t_{a}$ and $t_{b}$ are individually chosen for each $\dot{\gamma}_{a}$ by observing the time evolution of the flow field. In particular, these bursts were observed for imposed shear rates $\dot{\gamma}_{a}=18,20 \mathrm{~s}^{-1}$ and $\dot{\gamma}_{a}=15 \mathrm{~s}^{-1}$ (only rough wall). The nature of the bursts is discussed in section 6.3.2.2.

In general observations are similar to those for the NMR HR case (Fig. 6.3a,b and Fig. 6.4a,b). As expected a smaller amount of slip is present for the rough wall. Consequently a banded flow profile already develops at lower imposed shear rates as compared to the smooth wall. For the smooth wall, no banded structure is detectable at applied rates as high as $7 \mathrm{~s}^{-1}$, a value well within the stress plateau of the flow curve (Fig. 6.1). Interestingly, the velocity gradient is even closer to zero at the rotor wall for a shear rate of $5 \mathrm{~s}^{-1}$. It is likely that these observations for the smooth wall at the beginning of the stress plateau are caused by the competition of wall slip and the build-up of a high shear rate band as already pointed out by Fardin et al. (2012a). A tiny high shear rate band might be present, but is, however, not detectable with the given resolution and might also not be stable during the course of the experiment. For applied shear rates of $10 \mathrm{~s}^{-1}$ and higher a clear banding structure is found for both wall characteristics. 

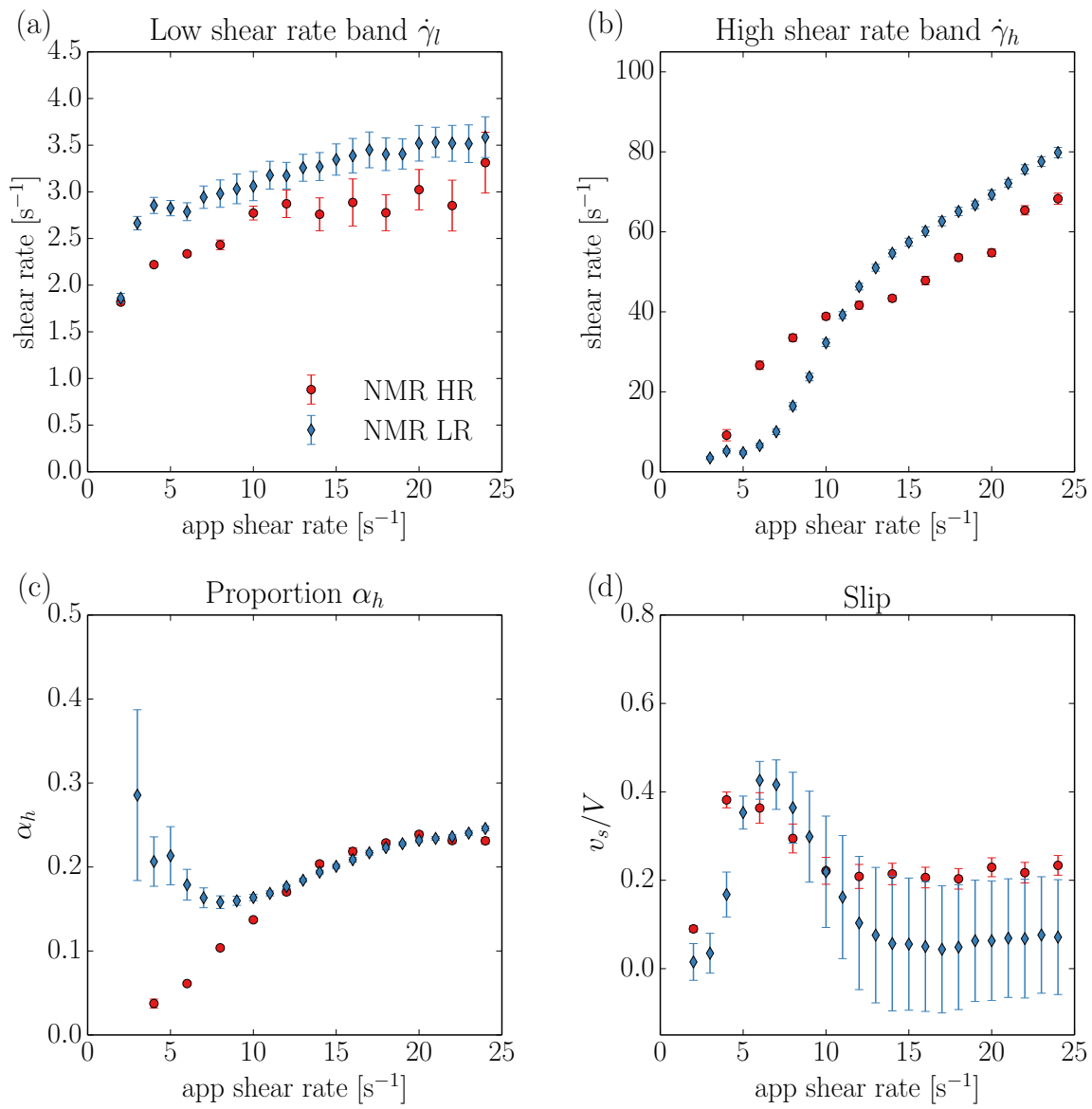

Fig. 6.5: Comparison of NMR LR and HR data. The parameters $\dot{\gamma}_{l}$ (a), $\dot{\gamma}_{h}$ (b), $\alpha_{h}$ (c), $v_{s} / V(\mathrm{~d})$ as obtained from the piece linear fits of the averaged velocity profiles are plotted as a function of the imposed shear rate $\dot{\gamma}_{a}$ The low resolution data does not capture the increase of $\alpha_{h}$ for $\dot{\gamma}_{a}<15 \mathrm{~s}^{-1}$. For $\dot{\gamma}_{a}>15 \mathrm{~s}^{-1}$ there is relatively good agreement between HR and LR. The errorbars for $\dot{\gamma}_{l}, \dot{\gamma}_{h}$ and $\alpha_{h}$ show the errors from the fit. The errorbars for the slip are given by deviations for the fluid velocity at the wall if one assumes a error of half a pixel width for the position of the inner wall. Please note, that errorbars are sometimes smaller than the size of the symbols.

Comparison of NMR and USV From the qualitative analysis of the averaged velocity profiles alone, it is obvious that for both experimental methods the fluid is not following a simple lever rule behaviour (Eq. (3.14)). A violation of this rule is in line with previous results on the same system by Feindel and Callaghan (2010) and Fardin et al. (2012a). As mentioned earlier however, the extent of the simple lever rule violation was different for each of the aforementioned references. In particular, Fardin et al. (2012a) reported on 

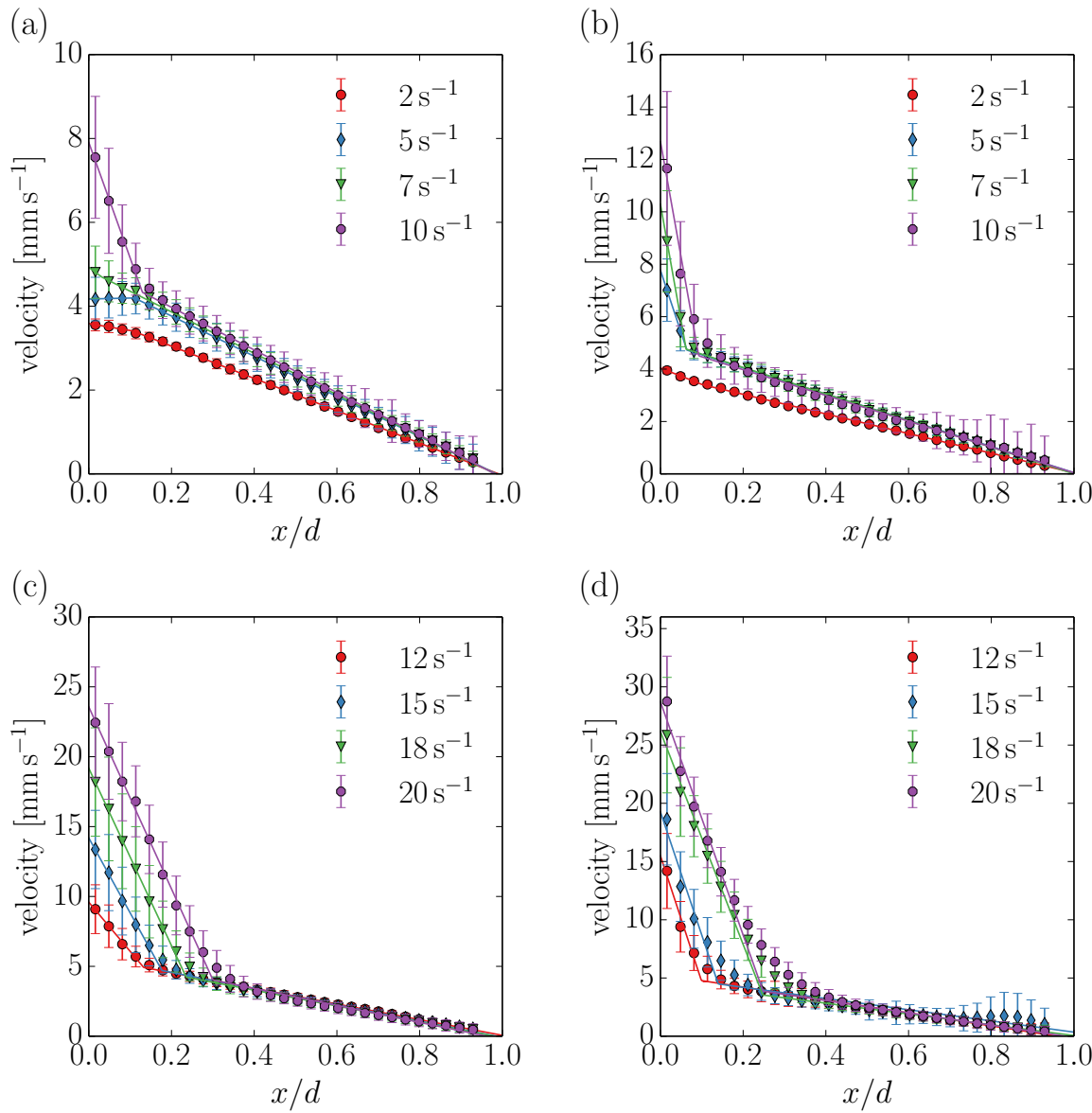

Fig. 6.6: Spatially and temporally averaged steady state profiles for USV measurements with smooth $(\mathrm{a}, \mathrm{c})$ and rough $(\mathrm{b}, \mathrm{d})$ inner wall surfaces at various imposed shear rates. The solid lines represent piece-wise linear fits of the flow profile. The average is taken over a window of $100 \mathrm{~s}$ starting $2.5 \mathrm{~min}$ after the inception of shear. Errorbars indicate the standard deviations and are measure for the amount of fluctuations present at the corresponding position. For $\dot{\gamma}_{a}>15 \mathrm{~s}^{-1}$ in the smooth case and $\dot{\gamma}_{a}>12 \mathrm{~s}^{-1}$ in the rough wall case turbulent bursts started altering the flow pattern after a certain time $t_{b}$. Therefore only a short period some time after the startup and $t_{b}$ is used for the average.

modifications and extensions of this rule in great detail. Three characteristic properties were identified that constitute an anomalous banding behaviour called standard anomalous lever rule. These three characteristics shall now be discussed in light of the new NMR and USV experiments whose findings are summarized in Fig. 6.7.

1. The first point is concerning the velocity gradient $\dot{\gamma}_{l}$ in the low shear rate band. It states that the value of $\dot{\gamma}_{1}$ marking the beginning of the stress plateau is a good estimate 
for $\dot{\gamma}_{l}$. This point can be regarded as fulfilled with a value of about $2.5 \mathrm{~s}^{-1}$ found both in the flow curve (Fig. 6.1) and in Fig. 6.7a for both techniques and wall surfaces. Slight fluctuations are observed for higher shear rates $\left(\dot{\gamma}_{a}>10 \mathrm{~s}^{-1}\right)$ which could be linked to a stronger influence of secondary flows on the overall banding behaviour. Also, essentially no slip on the outer wall of the Couette cell is apparent.

2. As a second characteristic, a linear increase of the high shear rate band proportion $\alpha_{h}$ with increasing $\dot{\gamma}_{a}$ has been identified by Fardin et al. (2012a). In contrast to point 1, differences between the three datasets are observed here. For USV smooth a linear increase of $\alpha_{h}$ over the range of studied shear rates is found and indicated by a linear fit (dashed line in Fig. 6.7c). On the other hand, the USV rough data shows bigger deviations with respect to the corresponding linear fit and exhibits a slight plateauing for $\dot{\gamma}_{a}=18 \mathrm{~s}^{-1}$ and $20 \mathrm{~s}^{-1}$. This might be explainable by the temporal window over which the average was taken (see previous paragraph). The band might not have reached its final size before turbulence started to dominate the flow. In the case of the NMR experiment, a linear increase is observed for $\dot{\gamma}_{a}<15 \mathrm{~s}^{-1}$. In this range of applied shear rates, the rate of change for $\alpha_{h}$ is also very similar to USV smooth, as the corresponding linear fit reveals. For higher imposed shear rates however, the proportion of the high shear rate band remains nearly constant. This might indicate that there is a considerable change in flow conditions for higher shear rates where elastic turbulence could play a role for the dynamics of the fluid. Yet, as shall be outlined in section 6.3.2.2, the NMR velocity profiles do not exhibit a direct indication of turbulent flow for these applied shear rates. Furthermore, the agreement between NMR HR and NMR LR in that $\dot{\gamma}_{a}$-range makes it unlikely that an experimental artefact particular to the high resolution method has led to this observation. In conclusion, the origin of this feature remains unexplained at this point and is further discussed later. Still, the linear increase observed for $\dot{\gamma}_{a}<15 \mathrm{~s}^{-1}$ is in line with the USV results and previous experiments by Fardin et al. (2012b).

3. The third and last point concerns the shear rate in the high shear rate band $\dot{\gamma}_{h}$, which was found not to be constant by Fardin et al. (2012b). As emphasized by the authors, this marks the main departure from the simple lever rule where $\dot{\gamma}_{h}$ takes a fixed value corresponding to the end of the stress plateau. Again, this feature is recovered in the present experiments as can be seen in Fig. 6.7b. Interestingly the particular dependence of $\dot{\gamma}_{h}$ on $\dot{\gamma}_{a}$ differs considerably among the three datasets. The USV rough measurements are characterized by a sharp increase to a value of $50 \mathrm{~s}^{-1}$ which then remains essentially constant. On the other hand an approximately linear increase for $\dot{\gamma}_{h}$ with increasing $\dot{\gamma}_{a}$ is observed for USV smooth. The NMR dataset contains features from both USV 
measurements. A sharp increase is followed by a linear section with a rate of change similar to USV smooth.
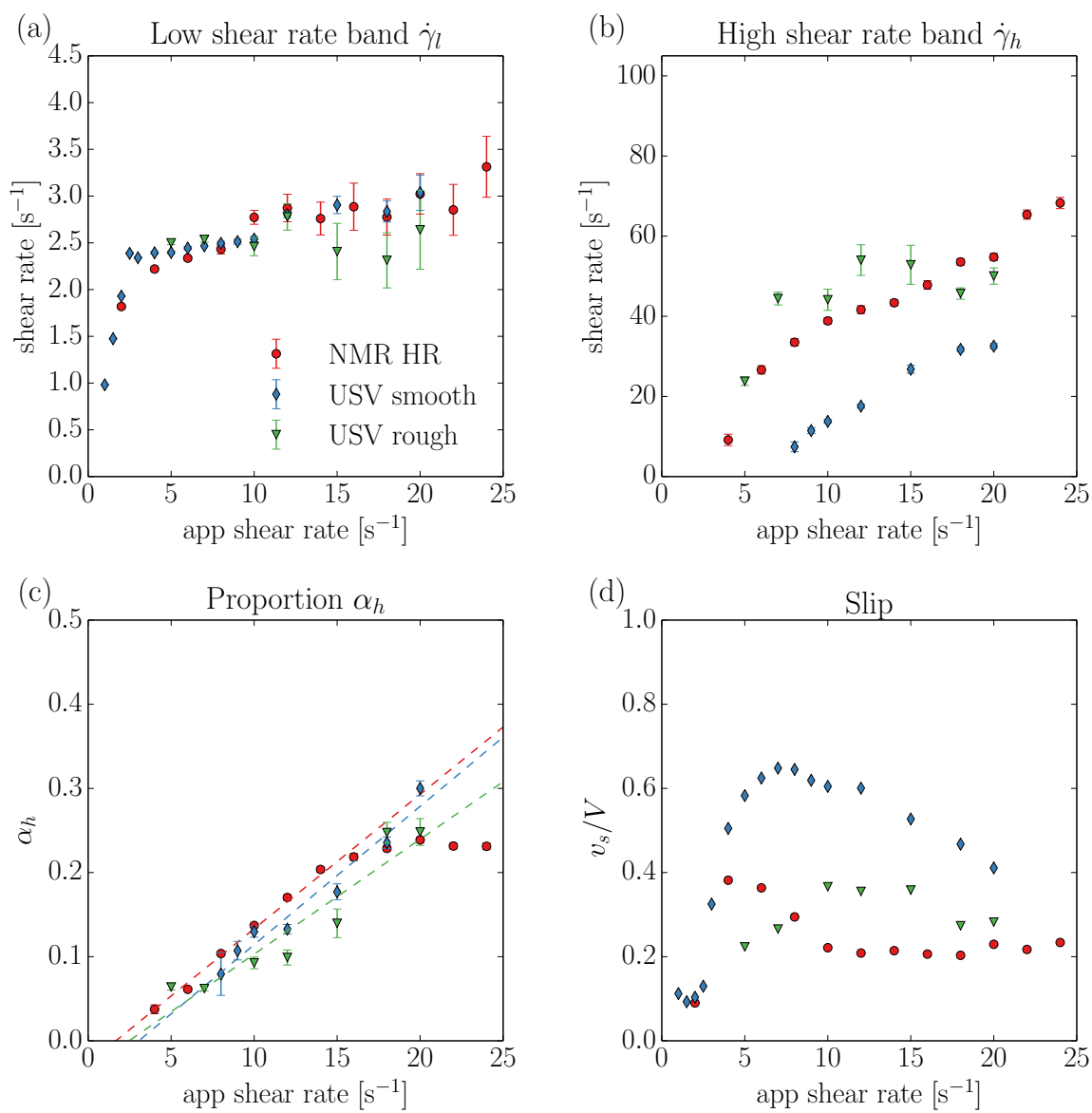

Fig. 6.7: Comparison of NMR HR and USV measurements. The parameters $\dot{\gamma}_{l}$ (a), $\dot{\gamma}_{h}$ (b), $\alpha_{h}$ (c), $v_{s} / V$ (d) as obtained from the piece-linear fits of the averaged velocity profiles are plotted as function of the imposed shear rate $\dot{\gamma}_{a}$. The errorbars indicate the fitting errors of the respective parameters, except for (d) (no errorbars). In (c) the dashed lines represent linear fits for different experimental condition (same colour coding). For NMR HR, only points where $\dot{\gamma}_{a}<17 \mathrm{~s}^{-1}$ are considered for the fit.

If the influence of turbulent bursts is ignored, then the extrapolation of the linear fits in Fig. $6.7 \mathrm{c}$ to $\alpha_{h}=1$ leads to estimates for the end of the stress plateau $\dot{\gamma}_{2}^{\prime}$. The shear rate values $\dot{\gamma}_{2}^{\prime}$ obtained from the extrapolation are in the range of $70 \mathrm{~s}^{-1}-80 \mathrm{~s}^{-1}$ and comparable to the findings by Fardin et al. (2012a) for the same system at a different temperature. In the case where the simple lever rule holds, the end of the stress plateau $\dot{\gamma}_{2}^{\prime}$ also corresponds to the shear rate $\dot{\gamma}_{h}$ in the low viscosity band. In the present experiments, however, $\dot{\gamma}_{h}$ 
is below this simple lever rule prediction for all applied shear rates. For example, the approximately constant value observed for USV rough (Fig. 6.7b) for $\dot{\gamma}_{a}>5 \mathrm{~s}^{-1}$ is about $30 \%$ lower than $\dot{\gamma}_{2}^{\prime}$. As this observation was also made by Fardin et al. (2012a), one can confirm the picture drawn by these authors and state that the fluid is indeed following a standard anomalous lever rule. The reasons as to why a different behaviour has been observed in the NMR experiments of Feindel and Callaghan (2010) include the different choice of slice thickness and $k$-space scheme and better control of thermal conditions in the present experiments.

Finally, the effect of slip shall be discussed. In general one can constitute that there is less slip for the NMR geometry. This is surprising as only a slightly sandblasted PEEK wall has been used. Additional NMR experiments, which are not shown here, have revealed that even for a smooth PEEK wall, the observed amount of normalized slip is below $30 \%$ which is in contrast to the smooth USV wall, where values up to $70 \%$ are found. One feature in Fig. 6.7d might provide a possible explanation for this discrepancy. For both NMR and USV smooth the slip reaches a maximum at about $5 \mathrm{~s}^{-1}$ before dropping to lower values. Yet, this feature is not obvious for USV rough where the normalized slip remains roughly at a constant value of $30 \%$. In fact, this is lower than what is observed for the NMR case around its maximum at $5 \mathrm{~s}^{-1}$. Therefore it might be reasonable to assume that for higher $\dot{\gamma}_{a}$ not only the surface characteristics of the inner rotating cylinder, but also other geometry specific measures (e.g. the fill height) considerably influence the slip behaviour.

Following the analysis in Fardin et al. (2012a), $\dot{\gamma}_{h}$ and $\alpha_{h}$ are plotted as a function of the true shear rate $\dot{\gamma}_{\text {true }}$ in Fig. 6.8. By defining $\dot{\gamma}_{\text {true }}=v(0) / d$, each applied shear rate is rescaled to an effective shear rate by subtracting the average slip apparent at the rotor. $v(0)$ denotes the average fluid velocity at the inner wall and $d$ the gap size. In theory, this should allow for better comparison across multiple experimental conditions. The velocity gradients in the high shear rate band (Fig. 6.8a) in the case of USV smooth fit better with the other two datasets as compared to Fig. $6.7 \mathrm{~b}$ where the applied shear rate was used. On the other hand, deviations for the interface position (Fig. 6.8b) are more pronounced in comparison to Fig. 6.7c. Hence, a mere rescaling of the imposed rates hardly decreases the differences observed. As already noted in Fardin et al. (2012a) the situation is more complicated and functional dependencies go far beyond the effect of average slip. 

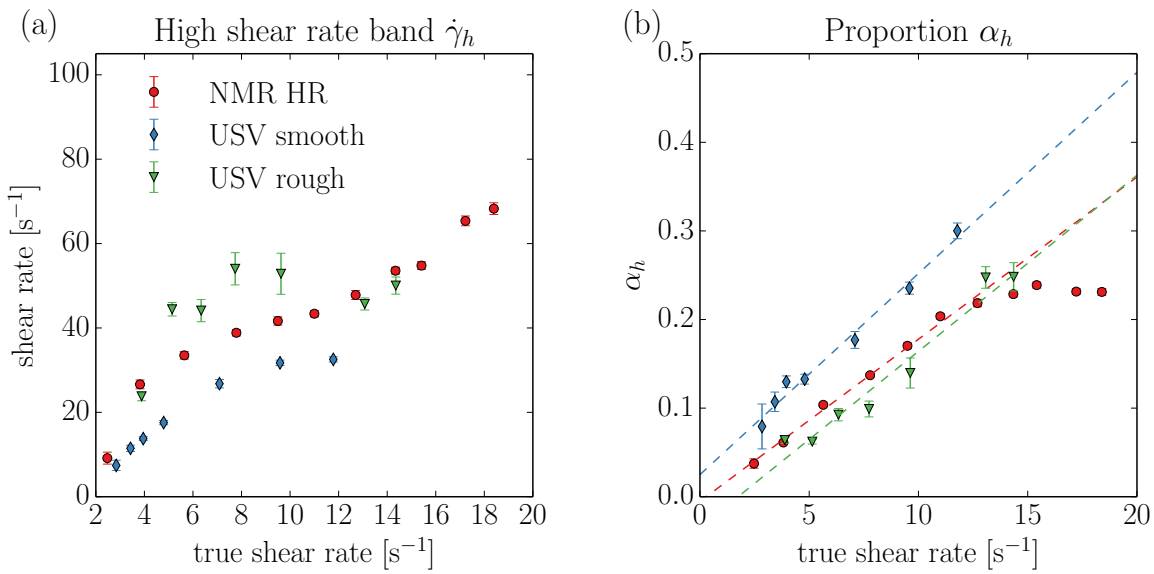

Fig. 6.8: (a) and (b) are the same as Fig. 6.7b and Fig. 6.7c, respectively, but with the true shear rate $\dot{\gamma}_{\text {true }}$ on the abscissa. For NMR HR in (b), only points where $\dot{\gamma}_{\text {true }}<10 \mathrm{~s}^{-1}$ are considered for the fit.

\subsubsection{Higher Dimensions: Vorticity Undulations and Temporal Fluctuations}

Space- and time averaged 1D velocity profiles give an impression on the general nature of the flow. However, these profiles might not reveal the underlying flow dynamics, which in turn can be characterized by instabilities and fluctuations. The aim of this section is to investigate the temporal and spacial structure of the flow in more detail with the help of the dimensional capabilities of the methods in use.

\subsubsection{Vorticity Undulations}

It has been shown recently that WLM solutions exhibit vorticity undulations while being sheared in a Couette cell (Fardin et al. (2009), Lerouge et al. (2008)). These structures in the axial direction resemble Taylor-Couette vortices, which have also been observed for polymeric fluids (Larson et al. (1990)). In a study including several different WLM solutions (Fardin et al. (2012b)) it was found, that the wavelength of these undulations scales linearly with the size of the high shear rate portion of the fluid, which is usually in the order of the gap width. In contrast to this finding, the authors Feindel and Callaghan (2010) observed structures in the order of centimetres.

Fig. 6.9 is representative of the vorticity structures typically observed for the experiments described here. In Fig. 6.9a (USV) and Fig. 6.9b (NMR) the temporal velocity evolution for a pixel close to the inner wall at an applied shear rate of $15 \mathrm{~s}^{-1}$ is shown 
as a function of the axial position $z$. Time averaging over the given period yields axial velocity profiles as depicted in Fig. 6.9c. The USV data clearly exhibits velocity undulation with a periodicity of a few millimetres. The NMR measurements on the other hand show a structure with a characteristic length in the order of the field of view $(\approx 25 \mathrm{~mm})$ similar to observations made by Feindel and Callaghan (2010). In Fig. 4b of Ref. Fardin et al. (2012b) a linear relation between the average proportion of the high shear rate band $\alpha_{h}$ and the wavelength of vorticity undulations $\lambda_{\text {und }}$ is established. This relation reads $\lambda_{\text {und }}=2 n \alpha_{h} d$, with $n=3.8$. By taking the values for $\alpha_{h}\left(\dot{\gamma}_{a}\right)$ at $\dot{\gamma}_{a}=15 \mathrm{~s}^{-1}$ from Fig. 6.7 (for USV) and Fig. 6.5 (for NMR), an expected wavelength of about $2.7 \mathrm{~mm}$ (USV) and $1.3 \mathrm{~mm}$ (NMR) can be calculated using the aforementioned linear relation. The difference between USV and NMR is mainly due to the different gap sizes $d$ for both methods. The undulations observed for the USV measurement appear to be close to the expectations. For the NMR measurements however, the expected wavelength is about the same size as the resolution in the vorticity direction $(1.56 \mathrm{~mm})$ and therefore not resolvable. Consequently, it might not be surprising that no clear undulation pattern is seen in the NMR case.
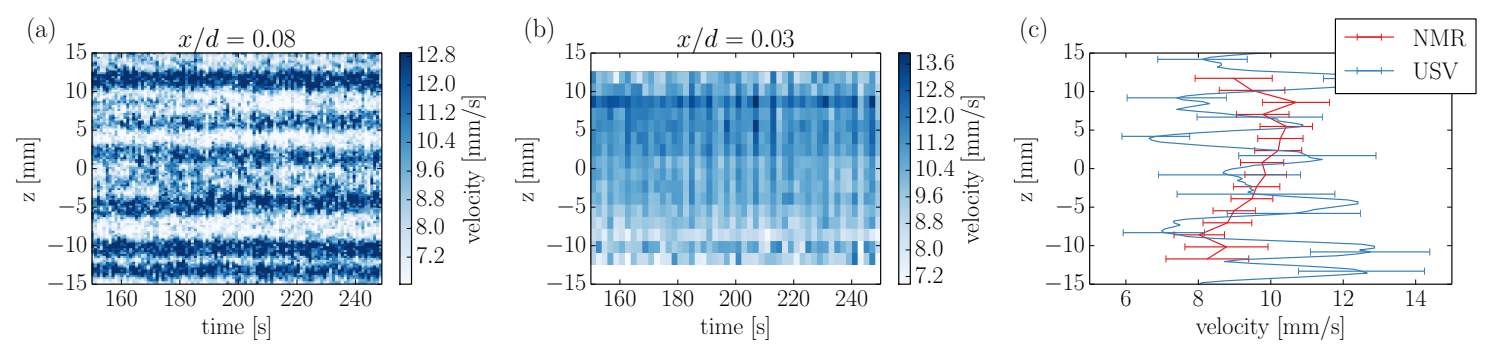

Fig. 6.9: Vorticity structure of the flow velocity for a position $x / d$ close to the inner rotating wall at $\dot{\gamma}_{a}=15 \mathrm{~s}^{-1}$ for USV smooth (a) and NMR LR (b). $x / d$ is given in the graph titles. In (c) the temporal average over the given time interval is shown. Shear inception started at time origin. Errorbars indicate standard deviations, only every tenth errorbar is shown for USV for better visualization. Note, that the field of view in the vorticity direction was about $30 \mathrm{~mm}$ for USV and $25 \mathrm{~mm}$ for NMR.

To study the USV undulations quantitatively, a procedure for the wavelength extraction has been developed. Firstly, the axial profile as shown in Fig. 6.9c is autocorrelated to smooth the velocity data allowing for an easier wavelength extraction. As a second step, the autocorrelated data are Fourier transformed to obtain a wavelength spectrum. Finally, the resulting wavelength is determined by picking the maximum of the spectrum. In Fig. 6.10, the procedure is shown step-wise for multiple datasets including shear rates 
of $10,12,15,18 \mathrm{~s}^{-1}$ for the smooth wall and $15 \mathrm{~s}^{-1}$ for the rough wall. For other combinations of $\dot{\gamma}_{a}$ and surface characteristic no clear undulation patterns could be found and a wavelength extraction was not possible. It shall be mentioned, that this method was preferred over a simple peak-to-peak measurement as the presence of multiple undulation wavelengths for single profiles is obvious from the wavelength spectra shown in Fig. 6.10 (third row).

The resulting wavelength from the datasets processed in Fig. 6.10 are depicted in Fig. 6.11. An approximately linear increase in wavelength with $\dot{\gamma}_{a}$ is observable. This is in line with findings from Fardin et al. (2012b). The wavelengths as such, however, are above the predictions by a factor of 2 . At this point it shall be mentioned that very similar results are obtained if the procedure is applied to $\alpha_{h}(z)$, the proportion of the high shear rate band resolved in the vorticity direction. $\alpha_{h}(z)$ is obtained for a certain $\dot{\gamma}_{a}$ by fitting each radial velocity profile of the corresponding time-averaged USV velocity map with a piece-wise linear function. The subsequent procedure is then identical to the case where the velocity information of a pixel close to the inner wall is used. This is exemplified for USV smooth at $\dot{\gamma}_{a}=15 \mathrm{~s}^{-1}$ in Fig. 6.12 , where the main peak in the wavelength spectrum is at the same position as in the corresponding plot in Fig. 6.10. However, it can be observed in Fig. 6.12 that the main peak is more pronounced in relation to the side peaks for the $\alpha_{h}(z)$ case. In terms of the temporal structure, it shall be noted that the vorticity pattern depicted in Fig. 6.9a remained steady over a time span longer than the one shown and can be categorized as steady vortex flow following Fardin et al. (2012b).

Finally, it should be mentioned that the predictions from Fardin et al. (2012b) should be seen as a rough guide. It can be expected that differences in the experimental setups lead to considerable changes in the observed wavelengths. Linear stability analysis and experiments have shown that a multitude of such wavelengths are possible under certain experimental conditions for a polymeric liquid (Larson et al. (1990)). Therefore, the existence of multiple wavelengths in the mm-range for our sample is also reasonable. Also, considerably different wavelengths for NMR and USV would not be surprising due to the differences in the geometries used. The characteristic length scale in the order of the field of view for the NMR measurements however, might be caused by experimental imperfections, as similar patterns are observed for pure water solutions imaged using the NMR technique. 

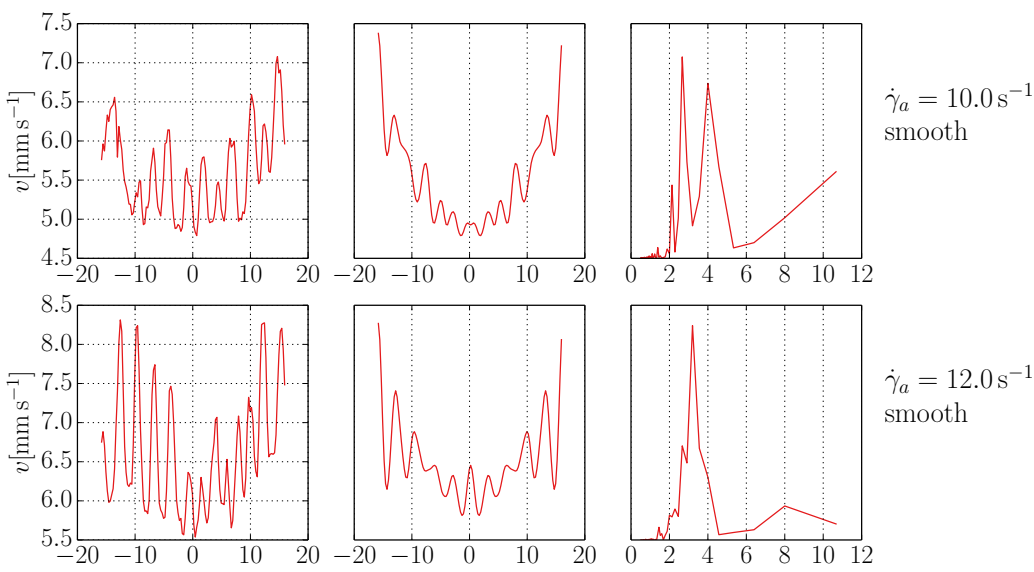

$\dot{\gamma}_{a}=12.0 \mathrm{~s}^{-1}$

smooth
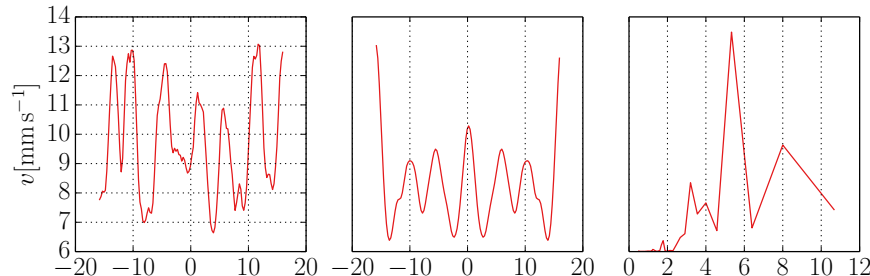

$\dot{\gamma}_{a}=15.0 \mathrm{~s}^{-1}$

smooth
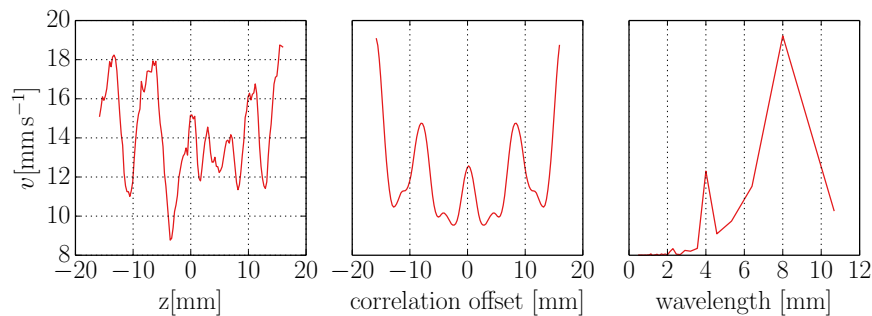

$\dot{\gamma}_{a}=18.0 \mathrm{~s}^{-1}$

smooth
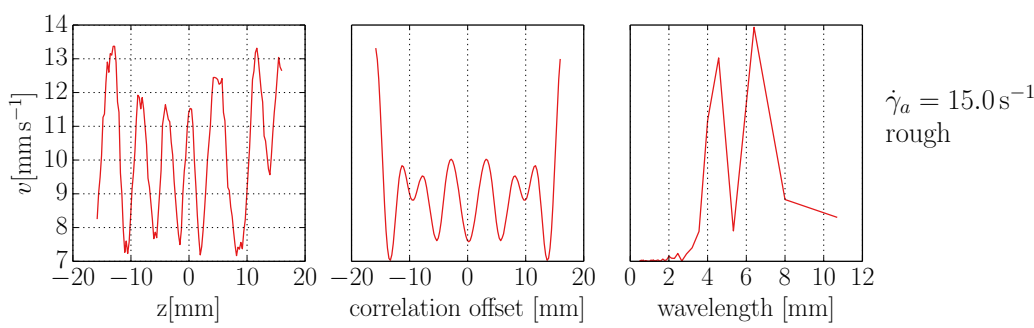

Fig. 6.10: The wavelength of vorticity undulations for USV smooth and USV rough was determined by Fourier transforming the autocorrelated velocity data for various shear rates. The first row shows the time-averaged axial velocity profile at a position close to the inner wall $x / d=0.08$ (same as in Fig. 6.9a). In the second row the corresponding autocorrelation function is depicted (arbitrary unit for ordinate). The third row shows the wavelength spectrum of the autocorrelation data which are truncated at $12 \mathrm{~mm}$ (arbitrary unit for ordinate). The undulation wavelength as shown in Fig. 6.11 is determined by picking the maximum of the spectral distribution. 


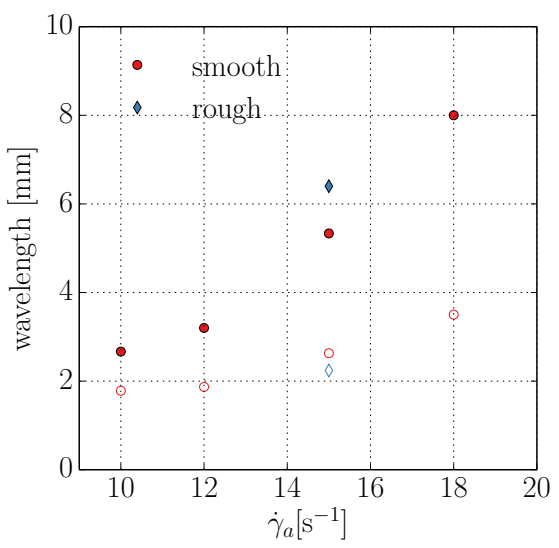

Fig. 6.11: Wavelengths extracted from Fourier spectrum analysis of the undulation pattern for time-averaged vorticity profile for a pixel close to the inner wall (filled symbols). See Fig. 6.10 for more details on the analysis procedure. As expected, the undulation wavelength shows an approximate linear dependence on the applied shear rates. Hollow symbols indicate the corresponding expected wavelengths obtained from a master curve in Fardin et al. (2012b).
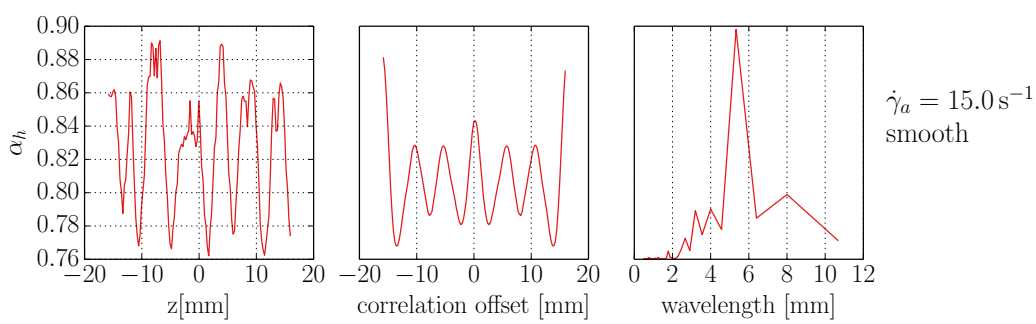

Fig. 6.12: Same as Fig. 6.10 for the $\dot{\gamma}_{a}=15 \mathrm{~s}^{-1}$ smooth case, but using the proportion of the high shear rate band $\alpha_{h}(z)$ instead of the velocity for a pixel close to the inner wall for the wavelength extraction.

\subsubsection{Turbulent Bursts}

In Fig. 6.13 the occurrence of a turbulent burst recorded with the high temporal resolution USV method (USV TH) at $\dot{\gamma}_{a}=18 \mathrm{~s}^{-1}$ is depicted. At a certain position along the vorticity direction (indicated by (i)) the banded flow becomes unstable over a period of about $300 \mathrm{~ms}$. The instability seems to originate from the high shear rate band, where a certain portion of fluid is expelled into parts of lower shear rate and thereby locally disturbs the structure of the shear banded base flow. The $2 \mathrm{D}$ flow pattern is similar to observation of Fardin et al. (2012a) with optical methods. Also the local velocity profiles during the 
burst (Fig. 6.13(i)) resemble those recorded with a 1D USV method in the aforementioned work. It should further be mentioned, that the recorded velocities during a turbulent burst might also be considerably affected by a non-zero radial velocity component, as outlined in section 6.2.2. This might explain the observation of negative velocities at time $2.92 \mathrm{~s}$ in Fig. 6.13(i). The profiles in Fig. 6.13(ii) and Fig. 6.13(iii) captured at two different position along the vorticity direction show a more stable flow pattern under the same time period, making clear that the burst is a local effect.
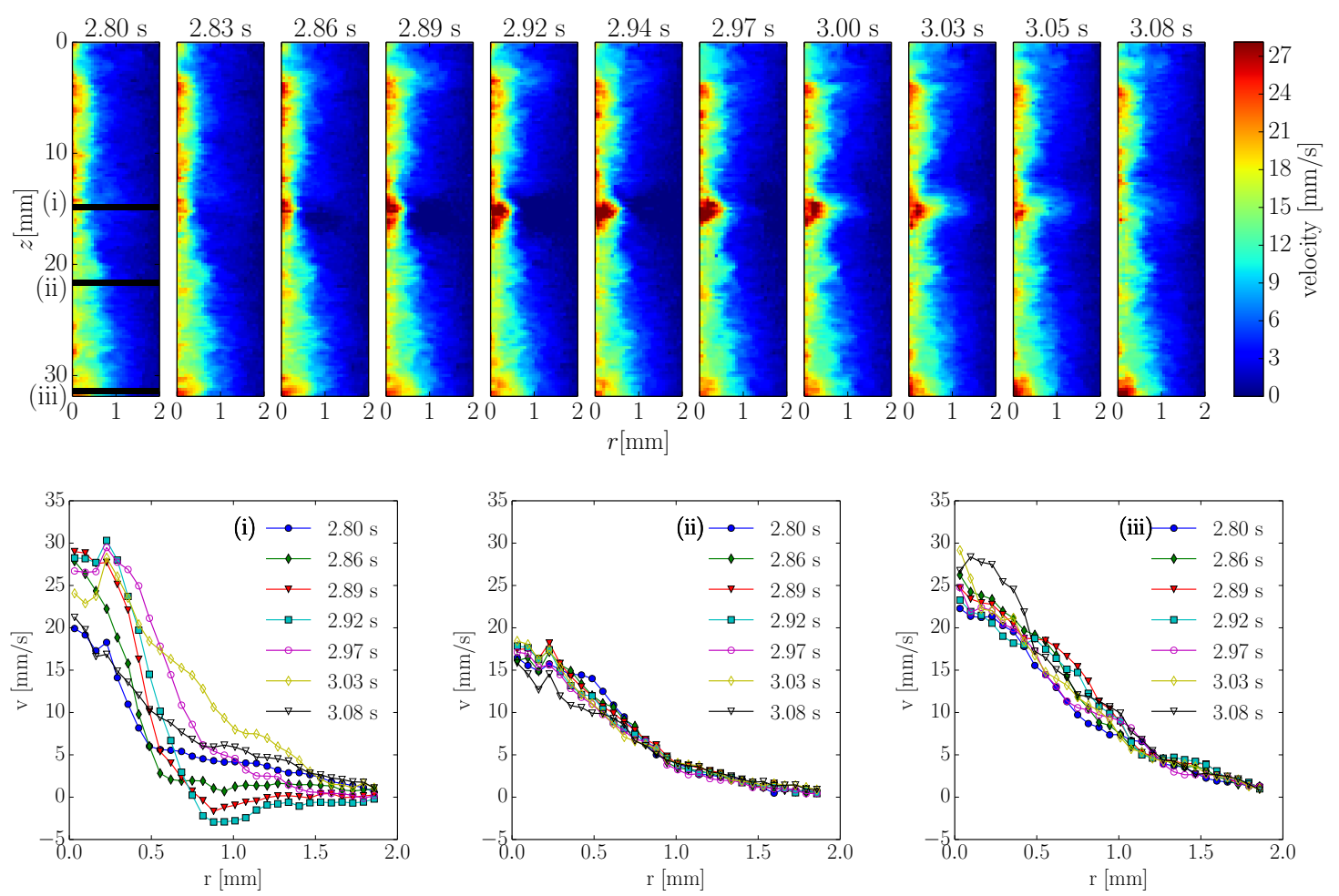

Fig. 6.13: Temporal evolution of the flow field over a short time period as recorded with USV TH at an imposed shear rate of $18 \mathrm{~s}^{-1}$ about $5 \mathrm{~min}$ after the startup. At position (i) the occurrence of a turbulent burst is captured. The flow of a certain fluid portion in the high shear rate band becomes unstable and massively alters the dynamics in its vicinity over the given time period. In general the flow is inhomogeneous in the vorticity and temporal directions even without bursts as exemplified by the profiles recorded at position (ii) and (iii).

In general, turbulent bursts start to occur in seemingly random intervals and at random axial positions after a certain time $t_{b}$ following the shear startup for high imposed shear rates. An inverse scaling of $t_{b}$ with $\dot{\gamma}_{a}$ has been observed. In the case of USV rough for example, bursts are detected $30 \mathrm{~s}$ after applying a shear rate of $20 \mathrm{~s}^{-1}$ whereas $t_{b} \approx 120 \mathrm{~s}$ 
for $\dot{\gamma}_{a}=15 \mathrm{~s}^{-1}$. No bursts were observed at shear rates lower than $15 \mathrm{~s}^{-1}$. The behaviour of the turbulent flow also depends on the wall characteristics. At an imposed shear rate of $15 \mathrm{~s}^{-1}$ the flow was stable and no bursts were detected for USV smooth. For $\dot{\gamma}_{a}=18,20 \mathrm{~s}^{-1}$ $t_{b}$ was higher as compared to the rough wall case.

In Fardin et al. (2012b) a criterion is developed (based on the work by Larson et al. (1990) and Pakdel and McKinley (1996)) that rationalizes the stability of the flow by a few quantities. The flow is expected to become unstable if the viscoelastic Taylor number

$$
\Sigma=\sqrt{\alpha_{h} \Lambda} \mathrm{Wi}_{\mathrm{h}}
$$

becomes greater than $m$, which is in the order of unity. $\Lambda$ is the geometrical ratio of gap size and rotor radius (Eq. (3.11)) and $\mathrm{Wi}_{\mathrm{h}}=\lambda \dot{\gamma}_{h}$ the local Weissenberg number in the high shear rate band, with $\lambda$ as the stress relaxation time of the fluid. In Fig. 6.14a $\Sigma$ is shown for NMR HR and both USV wall characteristics as a function of the applied shear rate. The values are calculated using the fit parameters $\alpha_{h}$ and $\dot{\gamma}_{h}$ of the averaged profiles presented in Fig. 6.7 and a value of about $500 \mathrm{~ms}$ for $\lambda$, which is extracted from previous results on the system by López-González et al. (2006). With respect to USV measurements (NMR case shall be discussed later), $\Sigma$ is generally higher at applied shear rates where bursts are observed as compared to applied shear rates where no bursts were detected. The only exception is the burst free flow for USV rough at $\dot{\gamma}_{a}=12 \mathrm{~s}^{-1}$ which exhibits a higher $\Sigma$ value than USV smooth at $18 \mathrm{~s}^{-1}$. According to these observations, a value for $m$ between 2 and 3 seems realistic and is also in the order of unity as predicted by the criterion.

Although it can be roughly confirmed that the criterion holds for the USV results, one has to be careful in general. Strictly speaking, this criterion can only be applied if the following conditions are fulfilled (Fardin et al. (2012b)). Firstly, the flow has to be free of inertial effects, i.e. at low Reynolds numbers Re. Secondly, influence of the bottom and top boundary condition has to be negligible. Thirdly, the small gap approximation has to be applicable, which means that $\Lambda \ll 1$. The first condition can be regarded as fulfilled for the range of shear rates explored here as shown by Fardin et al. (2012b). Also $\Lambda$ is small for the NMR and USV geometries, justifying the small gap approximation (see section 3.2.1.1). However, end effects could play an important role in the development of elastic turbulence as the top of the Couette cell is close to the active region of the measurement in the USV case. Furthermore, $\alpha_{h}$ and $\dot{\gamma}_{h}$ vary spatially and locally as found in section 6.3.2.1. Therefore, $\Sigma$ values can locally be above the stability threshold, although the mean is below in general. 

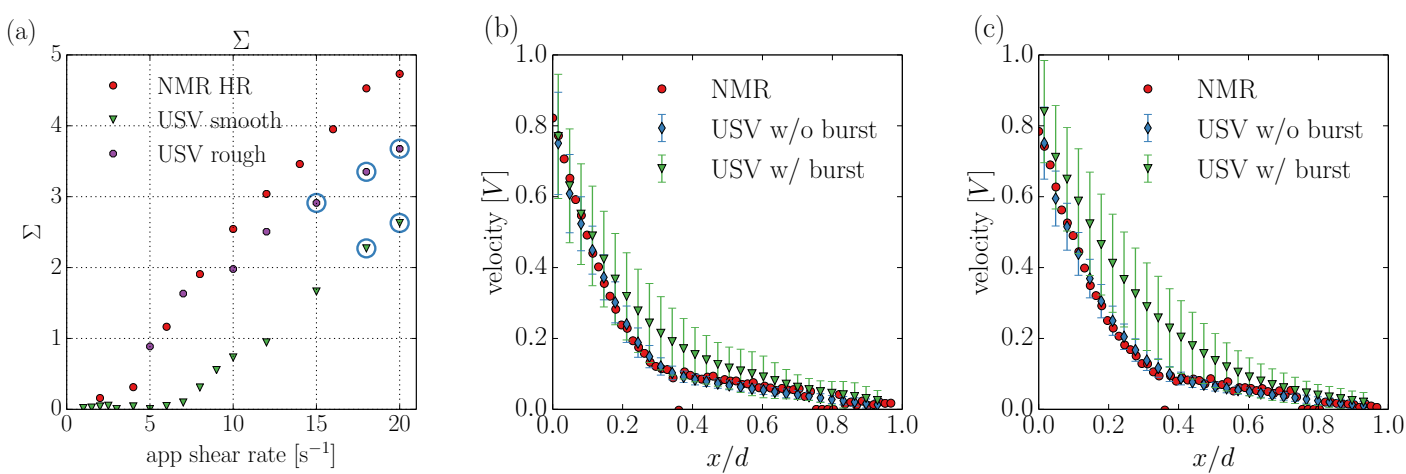

Fig. 6.14: (a) Instability criterion $\Sigma$ (Eq. (6.2)) calculated from the fit values of the averaged velocity profiles (see Fig. 6.7) for the USV and NMR HR experiments. $\Sigma_{0}$ corresponds to the $\Sigma$ value for USV rough at $\dot{\gamma}_{a}=15 \mathrm{~s}^{-1}$, the lowest applied shear rate where bursts are detected. The blue hollow circles indicate where bursts are detected. The stress relaxation time $\lambda$ is assumed to be equal in all three cases. (b)\&(c): Comparison of averaged flow profiles for shear rate of $18 s^{-1}$ (b) and $20 s^{-1}$ (c) for USV rough and NMR HR. Errorbars for USV indicate standard deviations. For each imposed shear rate the two USV rough profiles correspond to two different averaging windows, one without and one with the bursts. The profiles have been normalized by the rotor velocities $V$ allowing for better comparison of USV and NMR results.

In Fig. 6.14b,c the burst-free spatial and temporal averages at $\dot{\gamma}_{a}=18,20 \mathrm{~s}^{-1}$ for USV rough are compared to averages that also include profiles after the onset of turbulent bursts. The errorbars indicate the standard deviation for each pixel of the respective dataset. The highest deviations between the two averages are seen in the transition region between the bands. Also, the relative fluctuations are highest for the average including bursts in this region. This fits into the picture of instabilities first developing in the high shear band and then perturbing the flow of nearby regions in the low shear band. Furthermore, the effect of turbulent bursts becomes stronger for higher applied shear rates, as differences between the two profiles are more pronounced at $\dot{\gamma}_{a}=20 \mathrm{~s}^{-1}$ compared to $18 \mathrm{~s}^{-1}$. This is in line with observations from the spatially and temporally resolved data, where bursts appear more frequently in the $20 \mathrm{~s}^{-1}$ case.

Additionally the corresponding NMR HR averages are included in the two plots. They agree very well with the burst-free USV profile. Now it shall be recalled, that the NMR average is taken over almost $15 \mathrm{~min}$ after the transient start-up behaviour. That means, if the nature of the bursts was similar in both geometries the NMR profile should rather follow the average including bursts than the burst-free one. Yet the striking agreement with the latter strongly suggests that there are no bursts occurring during the NMR 
measurements, at least not in the active volume. This is surprising as $\Sigma$ is generally higher for NMR as compared to USV at the same applied shear rate, mainly due to the different dimensions of the NMR geometry $\left(\Lambda_{\mathrm{NMR}}=0.125\right.$ vs $\left.\Lambda_{\mathrm{USV}}=0.087\right)$ (see Fig. 6.14a). As mentioned however, the criterion can only be applied if end effects can be excluded. Therefore it is likely that the difference in boundary conditions at the top of the cell for NMR and USV is a major reason for the different observations. Note that the dimensionless aspect ratio $\Gamma=d / H$ (Eq. $(3.13))$ is smaller for $\operatorname{NMR}\left(\Gamma_{\mathrm{NMR}} \approx 0.008\right)$ as compared to USV ( $\left.\Gamma_{\mathrm{USV}} \approx 0.03\right)$ and therefore fluid instabilities triggered at the top of the cell might be more likely to intrude the active measurement region for the ultrasound setup. To this end, the levelling of $\alpha_{h}$ for the NMR experiments (see Fig. 6.7c) might be explained by turbulent flow in the top part of the cell. This instability would not directly be observable in the velocity profiles, but could have an impact on the overall flow dynamics in the whole cell. It shall be noted however, that additional NMR LR experiments with a modified top boundary condition, i.e. a plastic ring acting as a plug on top of the active volume similar to the USV setup, have not shown major signs of turbulent bursts either. Therefore, it is also likely that even subtle differences in the experimental setups can lead to widely different behaviour regarding elastic turbulence. Furthermore, the presence of the tiny glass spheres in USV solution might also decrease the stability of the flow and thus a triggering of turbulent bursts might be more likely at lower $\Sigma$ values.

\subsubsection{General Flow Fluctuations}

Apart from the strong disturbances of the banded flow by turbulent burst, considerable velocity fluctuations are generally present in the high shear rate band for all applied shear rates above the shear banding threshold, as can be seen in the standard deviation of the averaged radial profiles (see e.g. Fig. 6.4b,d). In order to get further insight into the nature of these fluctuations and to develop a better understanding of the characteristics extracted from the averaged profiles, each single radial profile (excluding initial transients and bursts) is fitted with a piece-wise linear function. Distributions for the main fit parameters in the NMR LR case are shown in Fig. 6.15a-d for various applied shear rates. As expected the distributions for $\dot{\gamma}_{l}$ (a) are relatively narrow, as no major fluctuations were observed in this band. On the other hand, the distributions for $\dot{\gamma}_{h}$ (b), $\alpha_{h}$ (c) and the slip (d) are wide underlining the fluctuative nature of the flow in the low viscosity band. Therefore, changes in the flow characteristics for varying $\dot{\gamma}_{a}$ as shown in Fig. 6.5 and Fig. 6.7 should be interpreted in terms of a shift of a broad distribution towards a different mean value. Thus, the mean radial velocity profile is not necessarily representative for the general flow 
behaviour. This idea is supported by the very good agreement of the distribution means and the parameters computed by fitting the averaged profiles (Fig. 6.15e-h). The increased standard deviation of the distribution for both $\dot{\gamma}_{l}$ (Fig. 6.15e) and $\dot{\gamma}_{h}$ (Fig. 6.15f) at higher shear rates might be caused by a stronger influence of secondary flows that affect both the low and the high shear rate band. The high standard deviations for $\alpha_{h}$ (Fig. 6.15g) at low applied shear rates are due to the fact that effectively only one data point is situated in the high shear rate band. Thus, a determination of an interface position is difficult for the fitting algorithm leading to the wide range of possible values. The unrealistic negative slip velocities observed in Fig. 6.15d are ascribed to uncertainties in the calibration procedure.

Furthermore, it shall be pointed out that experimental errors also have an impact on the widths of the distributions. Therefore, similar NMR LR measurements have been performed on the water sample used in section 5.3.2 to ensure that the broad distributions are not simply a result of the experimental noise. In order to mimic the average size of the high shear rate band for $\dot{\gamma}_{a}>15 \mathrm{~s}^{-1}$, only 3 pixels close to the rotor were fitted with a linear function for the shear rate extraction. It was found that the width of these distributions was only about one third compared to the micellar solution. Therefore, a significant part of the velocity fluctuations in Fig. 6.15 is assumed to be induced by the fluid flow itself.

The picture drawn by the NMR LR results is essentially reproduced when applying the analysis method to the ultrasound data as exemplified for USV smooth in Fig. 6.16. Relatively broad distributions are observed for all characteristics except for $\dot{\gamma}_{l}$ (Fig. 6.16e). At an applied shear rate of about $8 \mathrm{~s}^{-1}$, the high shear rate band can be resolved for the USV technique in the case of the smooth wall. In contrast to the NMR LR data, the standard deviation for $\dot{\gamma}_{h}$ is approximately constant for all values $\dot{\gamma}_{a}>8 \mathrm{~s}^{-1}$. On the other hand, the width of the $\alpha_{h}$ distributions increases slightly for higher rates, which was also observed in the NMR LR case.

Further analysis (not depicted here) has shown that the distributions remain wide even if the average is restricted spatially. Therefore, not only effects from steady vorticity structures but also local velocity fluctuations shape the distributions. USV data with high temporal resolution (USV TH) reveal that fluctuations happen on timescales of a few tens of milliseconds. The corresponding distributions are very similar to those obtained from USV TL. This is interesting as the velocity information is accumulated over the same time range (around $50 \mathrm{~ms}$ ) with only the repetition time $T_{\mathrm{R}}$ being different ( $1 \mathrm{~s}$ vs $\approx 25 \mathrm{~ms}$ ). Consequently the major characteristics of the fluctuations can be captured in a short time interval of a couple of seconds. At this point, it should be emphasized again that the NMR method is also sensitive to those rapid fluctuations. Although it takes a period 

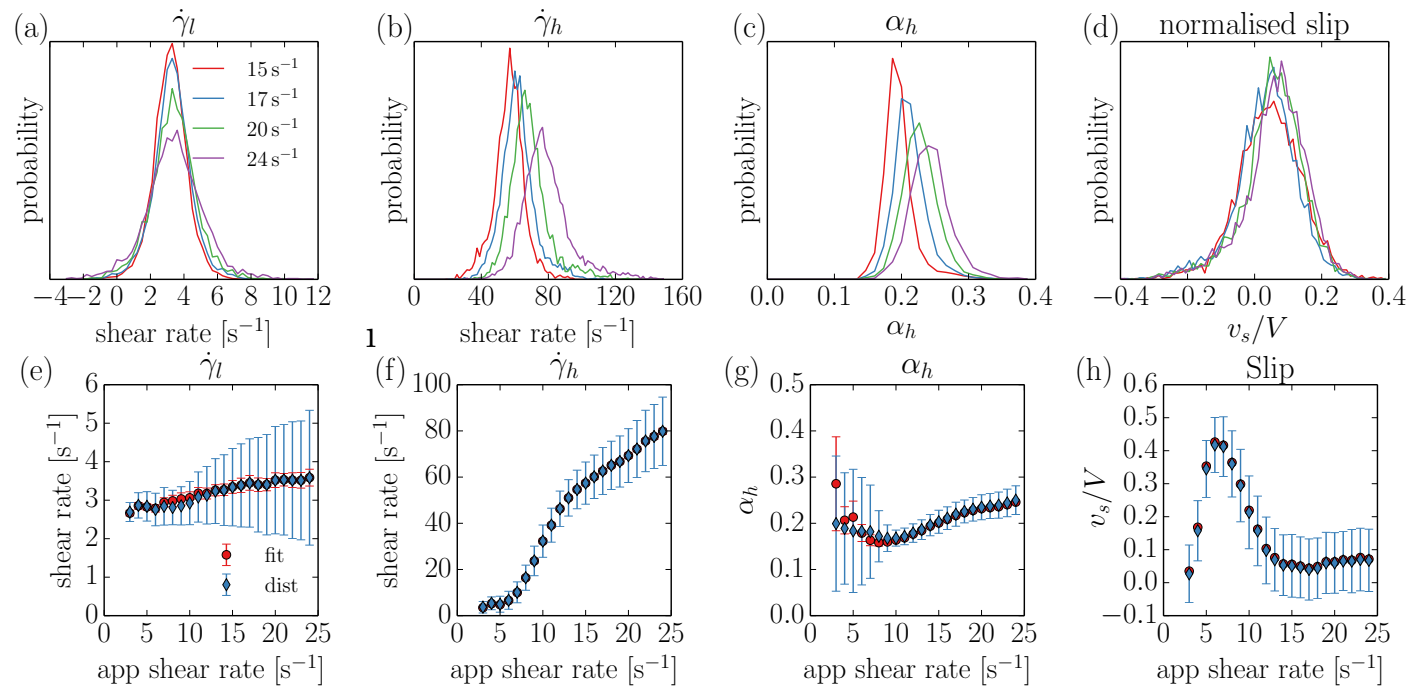

Fig. 6.15: Distributions for $\dot{\gamma}_{l}$ (a), $\dot{\gamma}_{h}$ (b), $\alpha_{h}$ (c) and the normalized slip (d) computed from the spatially and temporally resolved velocity profile fits for NMR LR at various applied shear rates. Broad distributions are obtained showing the fluctuative nature of the flow. In (e)-(h) the means of the distributions ("dist") are compared to the fits of the averaged velocity profiles ("fit"; from Fig. 6.5) for the whole range of imposed shear rates. The errorbars indicate the standard deviation of the distribution ((e)-(h)) and the fitting error (only (e)-(g)), respectively. The good agreement shows that the averaged velocity profiles represent the mean of a potentially broad distribution

of $2 \mathrm{~s}$ to generate a single flow map, the accumulation of velocity information only takes place over four $20 \mathrm{~ms}$ intervals as pointed out earlier. Consequently, each resulting velocity value is an average over a total time of $80 \mathrm{~ms}$. Hence, NMR and USV are comparable in the present situation regarding the velocity accumulation time for a single flow map.

Finally, it shall be reported that a Fourier analysis of the velocity time series data, as done by Brown and Callaghan (2011), have not revealed frequencies that could be linked to microscopic dynamics or timescales of the wormlike micellar solution. Most of characteristic frequencies found could be identified as harmonics of the rotational motion of the inner cylinder.

\subsection{Conclusions}

A combination of Rheo-NMR and Rheo-USV velocimetry has been used for a shear flow study of a $10 \% \mathrm{CPCl}$ WLM solution which has been the subject of various studies in recent years. The NMR methodology developed in the previous chapter made it possible 

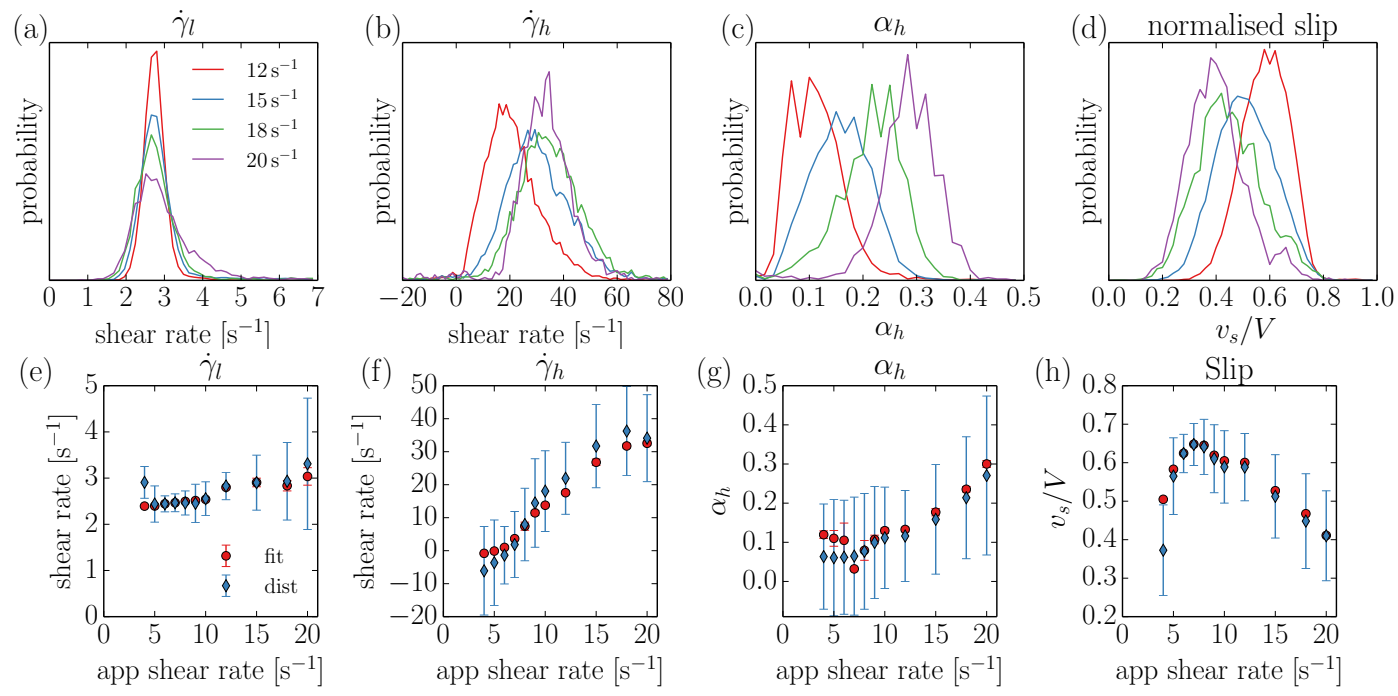

Fig. 6.16: Same as Fig. 6.15, but for the USV smooth experiments. The observations are similar to those obtained of the NMR LR case.

to draw a detailed picture of the dynamics of the flow in the shear banded state. The recently developed Rheo-USV technique allowed to capture the flow with very high spatial and temporal resolution. In particular, both methods confirm an anomalous lever rule behaviour similar to observations by Fardin et al. (2012a). It is characterized by a nearly constant shear rate in the low shear rate band and a variable shear rate in the high shear rate band. The proportion of the high shear rate band was found to linearly increase with the applied shear rate up to value of about $15 \mathrm{~s}^{-1}$ for NMR HR and USV. For higher values of $\dot{\gamma}_{a}, \alpha_{h}$ showed different behaviour depending on the shear cell and technique in use. These variations might be caused by a different nature of flow instabilities developing at such shear rates. Ultrasound measurements showed stable vorticity undulations for certain applied shear rates with wavelengths roughly in the range of those from previous studies. In the NMR case it is believed that the spatial resolution $(1.56 \mathrm{~mm})$ is insufficient to resolve these structures.

In the NMR measurements no direct evidence of turbulent bursts is found, whereas these flow features are unambiguously detected for the USV measurements. This is surprising as a criterion developed by Fardin et al. (2012b) predicts a more unstable flow for the NMR geometry at equivalent applied shear rates if end effects are ignored. However, these end effects seem to be important here. In particular, it is suspected that the fill height and the top boundary condition make the occurrence of turbulent bursts more likely in the USV case. As outlined by Fardin et al. (2012b) however, the nature of elastic turbulence is far 
from being understood thoroughly and needs further research. Finally, broad distributions are found if non-averaged profiles are fitted individually for both NMR and USV.

\section{Bibliography}

Jean-François Berret. Rheology of wormlike micelles: Equilibrium properties and shear banding transitions. In Richard G. Weiss and Pierre Terech, editors, Molecular Gels, pages 667-720. Springer Netherlands, January 2006. ISBN 978-1-4020-3352-0, 978-14020-3689-7. URL http://link.springer.com/chapter/10.1007/1-4020-3689-2_ 20.

Jean-François Berret, Denis C. Roux, and Gregoire Porte. Isotropic-to-nematic transition in wormlike micelles under shear. Journal de Physique II, 4(8): 1261-1279, August 1994. ISSN 1155-4312, 1286-4870. doi: 10.1051/jp2: 1994198. URL http://jp2.journaldephysique.org/articles/jp2/abs/1994/08/ jp2v4p1261/jp2v4p1261.html.

Melanie M. Britton and Paul T. Callaghan. Two-Phase shear band structures at uniform stress. Phys. Rev. Lett., 78(26):4930-4933, June 1997. doi: 10.1103/PhysRevLett.78. 4930. URL http://link.aps.org/doi/10.1103/PhysRevLett.78.4930.

Jennifer R. Brown and Paul T. Callaghan. Changing micellar order, lever rule behavior and spatio-temporal dynamics in shear-banding at the onset of the stress plateau. Soft Matter, 7(21):10472-10482, 2011. doi: 10.1039/C1SM06135F. URL http://dx.doi. org/10.1039/C1SM06135F.

M. E. Cates and S. M. Fielding. Rheology of giant micelles. Advances in Physics, 55 (7-8):799-879, 2006. ISSN 0001-8732. doi: 10.1080/00018730601082029. URL http: //dx.doi.org/10.1080/00018730601082029.

M. A. Fardin, B. Lasne, O. Cardoso, G. Grégoire, M. Argentina, J. P. Decruppe, and S. Lerouge. Taylor-like vortices in Shear-Banding flow of giant micelles. Phys. Rev. Lett., 103(2):028302, July 2009. doi: 10.1103/PhysRevLett.103.028302. URL http: //link.aps.org/doi/10.1103/PhysRevLett.103.028302.

M. A. Fardin, T. Divoux, M. A. Guedeau-Boudeville, I. Buchet-Maulien, J. Browaeys, G. H. McKinley, S. Manneville, and S. Lerouge. Shear-banding in surfactant wormlike micelles: elastic instabilities and wall slip. Soft Matter, 8(8):2535-2553, 2012a. doi: 10.1039/C2SM06992J. URL http://dx.doi.org/10.1039/C2SM06992J. 
M. A. Fardin, T. J. Ober, V. Grenard, T. Divoux, S. Manneville, G. H. McKinley, and S. Lerouge. Interplay between elastic instabilities and shear-banding: three categories of Taylor-Couette flows and beyond. Soft Matter, 8(39):10072-10089, September 2012b. ISSN 1744-6848. doi: 10.1039/C2SM26313K. URL http://pubs.rsc.org/en/ content/articlelanding/2012/sm/c2sm26313k.

Kirk Feindel, 2013. Published: personal communication.

Kirk Feindel and Paul Callaghan. Anomalous shear banding: multidimensional dynamics under fluctuating slip conditions. Rheologica Acta, 49(10):1003-1013, 2010. ISSN 00354511. URL http://dx.doi.org/10.1007/s00397-010-0470-9. 10.1007/s00397-0100470-9.

Thomas Gallot, Christophe Perge, Vincent Grenard, Marc-Antoine Fardin, Nicolas Taberlet, and Sébastien Manneville. Ultrafast ultrasonic imaging coupled to rheometry: Principle and illustration. Review of Scientific Instruments, 84(4):045107-045107-12, April 2013. ISSN 00346748. doi: doi:10.1063/1.4801462. URL http://rsi.aip.org/ resource/1/rsinak/v84/i4/p045107_s1?bypassSSO=1.

Matthew E. Helgeson, Paula A. Vasquez, Eric W. Kaler, and Norman J. Wagner. Rheology and spatially resolved structure of cetyltrimethylammonium bromide wormlike micelles through the shear banding transition. Journal of Rheology (1978-present), 53(3):727756, May 2009. ISSN 0148-6055, 1520-8516. doi: 10.1122/1.3089579. URL http: //scitation.aip.org/content/sor/journal/jor2/53/3/10.1122/1.3089579.

R. G. Larson, Eric S. G. Shaqfeh, and S. J. Muller. A purely elastic instability in Taylor-Couette flow. Journal of Fluid Mechanics, 218:573-600, 1990. doi: 10.1017/ S0022112090001124.

S. Lerouge, M. A. Fardin, M. Argentina, G. Grégoire, and O. Cardoso. Interface dynamics in shear-banding flow of giant micelles. Soft Matter, 4(9):1808, 2008. ISSN 1744-683X, 1744-6848. doi: 10.1039/b804915g. URL http://xlink.rsc.org/?DOI=b804915g.

Sandra Lerouge and Jean-François Berret. Shear-Induced transitions and instabilities in surfactant wormlike micelles. In Karel Dusek and Jean-François Joanny, editors, Polymer Characterization, number 230 in Advances in Polymer Science, pages 1-71. Springer Berlin Heidelberg, January 2010. ISBN 978-3-642-13531-6, 978-3-642-13532-3. URL http://link.springer.com/chapter/10.1007/12_2009_13. 
M. R. López-González, W. M. Holmes, P. T. Callaghan, and P. J. Photinos. Shear banding fluctuations and nematic order in wormlike micelles. Phys. Rev. Lett., 93(26):268302, December 2004. doi: 10.1103/PhysRevLett.93.268302. URL http://link.aps.org/ doi/10.1103/PhysRevLett.93.268302.

M. R. López-González, W. M. Holmes, and P. T. Callaghan. Rheo-NMR phenomena of wormlike micelles. Soft Matter, 2(10):855-869, September 2006. ISSN 1744-6848. doi: 10.1039/B600978F. URL http://pubs.rsc.org/en/content/articlelanding/2006/ $\mathrm{sm} / \mathrm{b} 600978 f$.

S. Manneville, L. Bécu, and A. Colin. High-frequency ultrasonic speckle velocimetry in sheared complex fluids. The European Physical Journal - Applied Physics, 28(03):361373, 2004. doi: 10.1051/epjap:2004165.

Robyn L. Moorcroft and Suzanne M. Fielding. Criteria for shear banding in TimeDependent flows of complex fluids. Physical Review Letters, 110(8):086001, February 2013. doi: 10.1103/PhysRevLett.110.086001. URL http://link.aps.org/doi/10. 1103/PhysRevLett.110.086001.

Peyman Pakdel and Gareth H. McKinley. Elastic instability and curved streamlines. Physical Review Letters, 77(12):2459-2462, September 1996. doi: 10.1103/PhysRevLett. 77.2459. URL http://link.aps.org/doi/10.1103/PhysRevLett.77.2459.

Allan Raudsepp and Paul T. Callaghan. A rheo-optical study of shear rate and optical anisotropy in wormlike micelles solutions. Soft Matter, 4(4):784-796, March 2008. ISSN 1744-6848. doi: 10.1039/B713416A. URL http://pubs.rsc.org/en/content/ articlelanding/2008/sm/b713416a.

Jean-Baptiste Salmon, Annie Colin, Sébastien Manneville, and François Molino. Velocity profiles in Shear-Banding wormlike micelles. Physical Review Letters, 90(22):228303, June 2003. doi: 10.1103/PhysRevLett.90.228303. URL http://link.aps.org/doi/ 10.1103/PhysRevLett.90.228303. 



\section{Summary and Outlook}

\subsection{Summary}

The main goal of this thesis was to further the understanding of shear banding with particular regard to spatial and temporal stability. To this end, the banded flow of a certain wormlike micellar solution (' $10 \%$ CPCl') sheared in a cylindrical Couette cell using NMR velocimetry has been investigated thoroughly. The NMR method of choice was a PGSE-RARE sequence which allows the acquisition of 2D flow maps with high temporal and spatial resolution. This sequence type had been used before for the study of the same micellar system (Feindel and Callaghan (2010)).

In order to ensure an accurate measurement of the Couette flow the methodology for the velocimetry sequence was refined and improved. Two main challenges were identified. The first relates to the fact that the flow in the studied Couette cell is of cylindrical nature, whereas the NMR imaging and slice selection process is bound to a Cartesian grid. The second is given by the small gap extent of the cells in question, which require high spatial resolution for a correct description of complicated flow dynamics. With regards to the first difficulty, it has been found that $1 \mathrm{~mm}$ is a suitable thickness for the slice selected in the PGSE part of the pulse sequence and Couette cell in use. As velocity values recorded in each voxel are always an average over the corresponding voxel volume, a wider slice leads to an underestimation of fluid velocities due to the stronger effect of the cell curvature. The study was supported by numerical calculations using model fluids and experiments with a micellar solution. Furthermore, the application of a centric scheme is recommended during the RARE part in order to minimize effects from in- and outflow during the $k$-space traversal. To this end, a computer program was developed allowing simulation of the signal generated by a PGSE-RARE sequence in the cylindrical Couette flow case. In similarity to the PGSE slice thickness case, considerable underestimation of fluid velocities is found if a linear $k$-space scheme is applied instead of a centric scheme, where the $k$-space centre is sampled in the beginning of the CPMG train. Again, these results were verified experimentally. It has also been shown that structures in the direction 
of the imaging phase gradient can be resolved despite the application of the centric scheme, which puts particular weight on the $k$-space centre and might therefore suppress finer image details. With regards to the second challenge, a high resolution variant of the PGSE-RARE pulse sequence has been developed, which allowed a resolution of up to $16 \mu \mathrm{m}$ in the read direction as compared to about $100 \mu \mathrm{m}$ for the conventional method. The main alteration concerned the introduction of a frequency offset for the signal readout resulting in a shifted field of view. This allowed reduction of the size of the latter, as effective suppression of resulting fold-over artefacts could be provided by frequency filters of the spectrometer. A small field of view was necessary for maintaining a reasonable sampling rate for the NMR signal in the high resolution case. Unfortunately, the acquisition of a $2 \mathrm{D} k$-space was not possible for the wormlike micellar solution due to the strong diffusive attenuation caused by the high imaging gradients. Hence, the reduction of the CPMG train to only two echoes was necessary effectively resulting in the measurement of $1 \mathrm{D}$ velocity profiles over the gap. On the other hand, the acquisition of 2D high resolution flow maps could be shown to work for a slowly diffusing polymeric system (PDMS). Additionally, with this PDMS system, the applicability of a recently suggested variation of the PGSE-RARE sequence (Shiko et al. (2012)) that allows maintainance of quadrature without the need for phase cycling has been proven. Further additions to the methodology include a procedure for the precise calibration of the gap position and a new Couette cell designed by a fellow $\mathrm{PhD}$ student (Tim Brox), which made it possible to use active temperature control via the Bruker temperature kit. A soft pulse simulation programmed during the course of this work helped in understanding an intensity scaling observed in the magnitude maps as well as spurious velocity measurements at the edges of the field of view.

Eventually, the methodology was used to draw a refined picture on the flow dynamics of the ' $10 \%$ CPCl' system. The study was supported by a recently developed 2D Rheo-USV velocimetry method (Gallot et al. (2013)) that offers even higher temporal resolution. With regards to averaged velocity profiles, both methods roughly confirm an anomalous lever rule behaviour similar to previous observations by Fardin et al. (2012a). This behaviour is characterized by a constant rate in the low shear rate band, a variable rate in the high shear rate band and a linearly increasing proportion of the latter band. In particular the increased resolution in the NMR HR measurements gave detailed insight into the dynamics of the interface between the two bands, which could not be recovered with the lower resolution method (NMR LR) over the whole range of investigated shear rates. The observation of a constant interface position for shear rates higher than $15 \mathrm{~s}^{-1}$ in the NMR case might be explained by the occurrence of turbulent bursts above the active volume that in turn could have influenced the flow dynamics in the active region. Ultrasound 
measurements show the presence of stable vorticity undulations for certain applied shear rates with wavelengths in the same order as for previous studies. In the NMR case it is believed that the spatial resolution was insufficient to resolve these structures. No direct evidence of turbulent bursts is found in the NMR measurements. In the USV case on the other hand, they are unambiguously detected at higher applied shear rates. This is surprising as a criterion developed by Fardin et al. (2012b) predicts are more unstable flow for the NMR geometry at equivalent applied shear rates if end effects are ignored. However, it is believed that these end effects play an important role in the present case causing the different behaviour regarding instabilities. It should be noted in any case, that the nature of elastic turbulence is far from being understood thoroughly today.

Finally, the results by Feindel and Callaghan (2010), which motivated this study, shall be brought to attention again. There, a lever rule inversion was observed, characterized by a constant interface position and changing shear rates in the bands. In the course of this thesis several challenges regarding the NMR measurement have been discussed, which might have affected the results in the aforementioned reference. The refined and further developed NMR methodology confirms an anomalous lever rule behaviour, however not a complete inversion of the rule as such. Furthermore, broad shear rate distributions in the high shear rate band caused by velocity fluctuations have been found. Therefore, a characterization of the microscopic fluid state based on averaged velocity profiles is regraded as being incomplete. In the present case, it is expected that the alignment of the wormlike micelles in the high shear rate band is also undergoing rapid fluctuations for a given imposed shear rate and is not locked in a fixed state as proposed by Feindel and Callaghan (2010). For the same reasons, shear history is not regarded to be important, as these rapid fluctuations should quickly erase any memory of past states in the fluid.

\subsection{Outlook}

NMR Simulation With regards to the RARE simulation program, a study with imperfect refocusing pulses (not $180^{\circ}$ ) would be of interest. In particular, it would be possible to trace different coherence pathways individually and estimate their significance for the acquired signal and resulting velocity measurement. In doing so, schemes might be advised that allow for the suppression of certain pathways that lead to coherences causing spurious signal contributions. Also, a further optimization of the simulation procedure for both programs might result in faster computation time. An ultimate goal in this respect would be a reasonable simulation time for the combination of both programs where slice selective pulses are actually simulated during the CPMG train of the RARE sequence. A 
plethora of possible studies would emerge from there, as, for example, the right choice of soft pulse shape or the effective slice thickness for the velocity measurement.

PGSE-RARE Methodology The methodology for the PGSE-RARE sequence allows for further refinement. For example, the significance of offsets in the selected slice could be investigated. In section 5.2.2.1 and section 5.2.3 it has been assumed that the selected slice is centred with respect to the Couette geometry. However, almost perfect alignment of the Couette cell and gradient system is hard to achieve. Therefore, the PGSE slice might be (slightly) off-centred and additional artefacts might be introduced. These could be investigated and methods advised of how to detect and avoid them by e.g. using frequency offsets to shift the slice centre.

Also, the relevance of the vanishing perpendicular magnetization component under the CPMG train might need further investigation with regards to the velocity measurement. For the first few echoes this component might still considerably contribute to the NMR signal and affect the recorded velocity value. In particular for the centric scheme, where the main image features are captured during those first echoes spurious coherences might be an issue. The application of a filter for the perpendicular component, as done by Ahola et al. (2006), might further help to eliminate unwanted signal. In the same vein, other $k$-space trajectories, like a shifted version of the centric scheme, could be tested and evaluated in regards to the accuracy of the flow measurement. With the help of the soft pulse simulation program the profile of the soft pulse could be optimized in order to minimize intensity scaling and spurious signal on the edges of the FOV. With respect to the $2 \mathrm{D}$ high resolution method, an interesting complex fluid sample exhibiting a low diffusion coefficient should be found, where the high spatial resolution of the method allows for new insights into its flow dynamics.

$10 \% \mathrm{CPCl}$ Vorticity undulations could not be observed due to the resolution of the NMR method in the direction of the imaging phase gradient. Increasing this resolution, by e.g. using a smaller field of view, could give better insight on the vorticity structure of the ' $10 \%$ CPCl' system for the NMR Couette cell. This investigation could be supported by a study on a WLM system which is known to exhibit more stable vorticity undulations, as for example described by Lerouge et al. (2008). If undulations can be detected in the latter case, there would be no doubt that these structures could also be resolved in ' $10 \%$ $\mathrm{CPCl}$ ' case, if present and not below the resolution limit. Another study could concern the application of the small step protocol as employed by Feindel and Callaghan (2010). Though there is no indication from this work that the shear protocol is considered to be 
of any importance for the particular WLM system, an additional study could give a more definite answer.

\section{Bibliography}

S. Ahola, J. Perlo, F. Casanova, S. Stapf, and B. Blümich. Multiecho sequence for velocity imaging in inhomogeneous rf fields. Journal of Magnetic Resonance, 182(1):143-151, September 2006. ISSN 1090-7807. doi: 10.1016/j.jmr.2006.06.017. URL http://www. sciencedirect.com/science/article/pii/S1090780706001765.

M. A. Fardin, T. Divoux, M. A. Guedeau-Boudeville, I. Buchet-Maulien, J. Browaeys, G. H. McKinley, S. Manneville, and S. Lerouge. Shear-banding in surfactant wormlike micelles: elastic instabilities and wall slip. Soft Matter, 8(8):2535-2553, 2012a. doi: 10.1039/C2SM06992J. URL http://dx.doi.org/10.1039/C2SM06992J.

M. A. Fardin, T. J. Ober, V. Grenard, T. Divoux, S. Manneville, G. H. McKinley, and S. Lerouge. Interplay between elastic instabilities and shear-banding: three categories of Taylor-Couette flows and beyond. Soft Matter, 8(39):10072-10089, September 2012b. ISSN 1744-6848. doi: 10.1039/C2SM26313K. URL http://pubs.rsc.org/en/ content/articlelanding/2012/sm/c2sm26313k.

Kirk Feindel and Paul Callaghan. Anomalous shear banding: multidimensional dynamics under fluctuating slip conditions. Rheologica Acta, 49(10):1003-1013, 2010. ISSN 00354511. URL http://dx.doi.org/10.1007/s00397-010-0470-9. 10.1007/s00397-010$0470-9$

Thomas Gallot, Christophe Perge, Vincent Grenard, Marc-Antoine Fardin, Nicolas Taberlet, and Sébastien Manneville. Ultrafast ultrasonic imaging coupled to rheometry: Principle and illustration. Review of Scientific Instruments, 84(4):045107-045107-12, April 2013. ISSN 00346748. doi: doi:10.1063/1.4801462. URL http://rsi.aip.org/ resource/1/rsinak/v84/i4/p045107_s1?bypassSSO=1.

S. Lerouge, M. A. Fardin, M. Argentina, G. Grégoire, and O. Cardoso. Interface dynamics in shear-banding flow of giant micelles. Soft Matter, 4(9):1808, 2008. ISSN 1744-683X, 1744-6848. doi: 10.1039/b804915g. URL http://xlink.rsc.org/?DOI=b804915g.

G. Shiko, A.J. Sederman, and L.F. Gladden. MRI technique for the snapshot imaging of quantitative velocity maps using RARE. Journal of Magnetic Resonance, 216:183- 
191, March 2012. ISSN 1090-7807. doi: 10.1016/j.jmr.2012.01.021. URL http://www . sciencedirect.com/science/article/pii/S1090780712000560. 


\section{List of Figures}

2.1 Zeeman diagram for a nuclei with $I=\frac{1}{2}$. The lower energy state has a slightly higher probability of being occupied. . . . . . . . . . . . 6

2.2 (a) Arbitrary magnetization vector $\mathbf{M}$ precessing around the axis of the static magnetic field $\mathbf{B}_{0}$ ( $z$-axis) in the laboratory frame. (b) Static magnetization vector $\mathbf{M}$ in the rotating frame in the on-resonant case. The angle $\varphi$ denotes the phase of the magnetization. . . . . . . . . . . . . . .

2.3 The effect of $90^{\circ}-x$ (a), $90^{\circ} y$ (b) and $180^{\circ}$ (c) pulses on an initial magnetization vector oriented in the $z$-direction. The $x$ - and $y$-axis refer to the (on-resonant) rotating frame. For (a) the rotation axis $\mathbf{e}_{\text {rot }}$, the rf phase $\phi$ and the rotation angle $\theta$ are also indicated. . . . . . . . . . . . 9

2.4 Acquisition of an FID shown as pulse program diagram. Fourier transform of the acquired signal leads to frequency spectrum. . . . . . . . . . . . . . 11

2.5 (i) Pulse sequence used for the generation of a spin-echo. (ii) Behaviour of the magnetization under this pulse sequence for two different $180^{\circ}$ pulse phases. The magnetization initially in thermal equilibrium (1) is tipped into the transverse plane (2) by applying an excitation pulse. Due to field inhomogeneity the magnetization dephases, which can be understood as a broadening of the magnetization vector (3). The application of a $180^{\circ}$ refocusing pulse inverts the phase relation with respect to the rotational axis (4a: $-x, 4 \mathrm{~b}: y)$. After the refocusing pulse, off-resonance effects act in the same manner and thereby rephase the magnetization $(5 \mathrm{a}, 5 \mathrm{~b})$. At the echo time $T=2 \tau$ the magnetization is coherent again and forms a spin echo $(6 \mathrm{a}, 6 \mathrm{~b})$. Please note that the resulting magnetization vectors in case (6a) and $(6 \mathrm{~b})$ are antiparallel. . . . . . . . . . . . . . . 15 
2.6 CPMG train: An array of $180^{\circ}$ refocusing pulses is used to continually undo off-resonant effects caused by field inhomogeneities. At each integer multiple of $T=2 \tau$ a spin echo is formed during the train. The envelope of the echo amplitude is given by the $T_{2}$ decay if other relaxation mechanism can be neglected. . . . . . . . . . . . . . . . . . . . . 1

2.7 Stimulated echo: The magnetization is first brought into the transverse plane by the initial $90^{\circ}$ pulse, where it dephases over a period $\tau$. The application of a second $90^{\circ}$ pulse transfers a certain portion of the magnetization in the longitudinal direction, where it is 'stored' over period $T_{\text {store. }}$ The application of another $90^{\circ}$ pulse brings the stored magnetization back into the transverse plane where an echo is formed after another period of length $\tau$. Please note, that a simple two vector model as employed for the spin echo is not sufficient for explaining the formation of a stimulated echo. . . . . . . 18

2.8 CPMG sequence with a variable phase angle $\phi$ and nutation angles of $\alpha$ for the refocusing pulses. . . . . . . . . . . . . . . 18

2.9 A slice of thickness $\Delta z$ is selected out of a cylindrical object. . . . . . . . . 21

$2.102 \mathrm{D}$ matrix for $k$-space (a) and real space (b). Please note, that equivalent stepping intervals $\Delta k_{1}$ and $\Delta k_{2}$ in $k$-space lead to an equivalent FOV in both spatial directions. However, the resolutions $\Delta l_{1}$ and $\Delta l_{2}$ are different due to different values for $k_{1}^{\max }$ and $k_{2}^{\max } \ldots \ldots \ldots \ldots 2$

2.11 The PGSE (pulsed gradient spin echo) sequence: Two gradients of equal length $\delta$, strength $g$ and direction with a refocusing $180^{\circ}$ pulse in between. The temporal spacing between the two gradient pulses $\Delta$ is called observation time, whereas $\delta$ is known as encoding time. . . . . . . . . . 26

2.12 Rapid acquisition relaxation enhanced (RARE) sequence shown along with two different $k$-space trajectories (linear and centric). Black means zero gradient strength for the acquisition schemes. . . . . . . . . . . . . 28

2.13 A variant of the EPI sequence along with the employed $k$-space trajectory. . 30

3.1 The application of a constant shear stress $\sigma$ causes a deformation of the solid object such that $\sigma=l / L$. Upon stress cessation, the solid block returns to its original state. . . . . . . . . . . . . . . . .

3.2 A Newtonian liquid is sheared between two parallel planes with relative velocity $V$ that are separated by a distance $d$. The velocity gradient $\dot{\gamma}$, known as shear rate, is given by $\dot{\gamma}=V / d$ in this case. . . . . . . . . . . 39

3.3 Stress tensor acting on a finite volume element. . . . . . . . . . . . . . . . . 42 
3.4 Schematic of a cylindrical Couette cell consisting of two concentric cylinders. Here the inner cylinder (rotor) is rotated with an angular velocity of $\Omega$ while the outer cylinder is stationary (stator). This imposes a shear motion on the sample fluid which is situated in the gap between both cylinders. . . . 43

3.5 Gap velocity profiles $v_{\varphi}(r)$ in the case of a Newtonian fluid for two cylindrical Couette cells with different radii $R$ and gap sizes $d$ (given in the plot titles). The velocity of the rotor is $V$. Also shown are linear profiles $v_{\text {lin }}(r)$, which would be expected in the corresponding simple shear case, as well as the difference between $v_{\operatorname{lin}}(r)$ and $v_{\varphi}(r)$ (multiplied by 10). For the cell with the smaller geometrical ratio $\Lambda$ (a), the velocity profile $v_{\varphi}(r)$ shows only slight deviations from the simple shear case whereas differences are considerable for the cell in (b). The plot legend applies to (a) and (b). . . .

3.6 Two shear geometries commonly used in rheology: (a) Cone-and-plate (b) Plate-plate . . . . . . . . . . . . . . . . 4 46

3.7 Schematic flow curves for Newtonian and common non-Newtonian fluids. . 47

3.8 Schematic phase diagram for a surfactant solution. The cubic boxes indicate the different concentration regimes in the case of a wormlike micellar solution. 49

3.9 Dependency of micellar structure on critical packing parameter (adapted from Larson (1999)). . . . . . . . . . . . . . . . . . . . 50

3.10 Schematic representation of a wormlike micelle with characteristic lengthscales: the radius of gyration $R_{g}$, the contour length $L$, the cross-section diameter $D_{C S}$ and the persistence length $l_{p}$. Adapted from Dreiss (2007). . 51

3.11 (a) Schematic of the underlying constitutive relation (solid line) and the observed flow curve (dashed where different) for a shear banding micellar solution. The shear rates $\dot{\gamma}_{1}$ and $\dot{\gamma}_{2}$ mark the beginning and the end of the stress plateau. (b) The expected flow field for a shear banding solution following the simple lever rule (Eq. (3.14)) at an applied shear rate $\dot{\gamma}_{a}$ within the stress plateau. The emergence of a low viscosity band is associated with a phase change from an isotropic to a nematic state. $d$ is the distance between the plates. . . . . . . . . . . . . . . . . . . . 54

4.1 Horizontal (a) and vertical (b) cuts through the input geometry for the simulation program. The dark blue sections mark the active volume. Dashed lines indicate the slice. Please note that for slice thicknesses $s<r_{2}$, the angle range $\Delta \varphi$ used for the rasterization is depending on the radial position r................................ 60 
4.2 Horizontal cut through the active volume as displayed by the graphical user interface of the simulation program (see Fig. 4.1a). The control fields on the right hand side can be used to adjust simulation specific parameters. . .

4.3 (a) Close-up on grid point $j . A$ is the area of the corresponding area element, whereas $\mathrm{d} r$ and $\mathrm{d} \varphi$ are the stepsizes in the cylindrical grid. (b) Deformed grid in accordance to the (purely tangential) flow of the simulated fluid. Each point in the grid is treated as an isochromat under the application of a gradient pulse. Please note, that the grid density is lower as compared to Fig. 4.1 for better visualization. . . . . . . . . . . . . . . . .

4.4 (a) The acquired signal $S(t)$ (shown: real part) is the sum over the magnetization of all isochromats at $t$. A RARE-type sequence was used in the present case. The values $N_{r}, N_{\varphi}$ and $N_{z}$ were all set to 50. (b): MR image of the Couette gap computed by Fourier transformation of the acquired signal from (a) . . . . . . . . . . . . . . . . . . . . . . 64

4.5 Pulse sequence for the simulation program. . . . . . . . . . . . 67

4.6 'sinc3G10' pulse and Gaussian envelope. . . . . . . . . . . . . . . . . . . . 69

4.7 (a) Comparison of simulated signal obtained with the sequence in Fig. 4.5 and the signal obtained from an equivalent 'imslice' experiment with the 'sinc3G10' pulse. Note, that the simulation signal is scaled such that the signal maximum coincides with that of the experiment. (b) 1D intensity profiles for simulation and experiment obtained by Fourier transform of the recorded signal from (a). The thickness of the slice is denoted by $s$. Similarly to (a), the simulation profile was scaled such that the maximum equals the one of the experiment. . . . . . . . . . . . . .

5.1 Schematic of the experimental setup. A. Strain controlled stepper motor. B. Drive shaft connecting motor and Couette cell. C. Shell of superconducting magnet. D. Cylindrical Couette cell. E. Imaging region. F. Rf coil. G. Access for air flow. . . . . . . . . . . . . . . . . . 75

5.2 (a) Sketch of the Taylor-Couette cell B used for the NMR experiments. The active region is indicated by the dashed rectangle. (b) Side view of the same cell. The upper dashed line corresponds to a typical fill height for the fluids under study. Also, measures at the bottom of the Couette cell are given and the centre of the coil and gradient system in the axial direction is indicated . . . . . . . . . . . . . . . . . . 77 
5.3 PGSE-RARE sequence used to record 2D velocity maps. Also shown are two different $k$-space trajectories, namely centric and linear. Black means no (phase) gradient. . . . . . . . . . . . . . . . . . . . 77

5.4 Schematics of vertical (a) and horizontal (b) cuts through the cylindrical Couette geometry. The active volume/region given by the slice selection of the NMR pulse sequence is indicated by the dashed rectangle. The Teflon band shown in (a) is used for cell A to create a (flow) feature in the phase

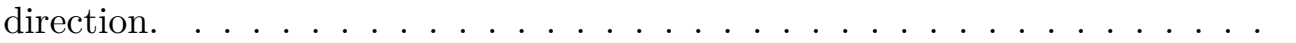

5.5 Typical outcome of the 2D PGSE-RARE experiment for cell B: Magnitude information (a) and flow map (b) resulting from phase contrast. Read and phase correspond to $x$ and $z$ respectively as defined in Fig. 5.4. The field of view is $20 \mathrm{~mm}$ in read and $25 \mathrm{~mm}$ in the phase direction. Deviation in the MRI image and the flow map at the top and bottom edge as well as an intensity scaling in the phase direction in (a) are due to the slice selection in the RARE part of the sequence. . . . . . . . . . . . . . . . . 80

5.6 The flow map in Fig. 5.5b as a waterfall plot showing individual 1D flow profiles along the phase direction. . . . . . . . . . . . . . . . .

5.7 (a) and (c) show flow maps of a model fluid exhibiting a linear profile over the gap at an imposed shear rate $\dot{\gamma}_{a}=10 \mathrm{~s}^{-1}$ confined to a cylindrical Couette geometry with equivalent dimensions as the recently developed NMR cell. The view is restricted to the active volume for slice thicknesses of $4 \mathrm{~mm}$ (a) and $1 \mathrm{~mm}$ (c). In (b) and (d) the average velocity in each voxel (dots) is shown for a range of applied shear rates. Vertical dotted lines indicate the voxel boundaries in each plot and solid lines are linear fits taking only points within the gap range $(8 \mathrm{~mm}-9 \mathrm{~mm})$ into account. The dashed lines indicate the assumed velocity profile. Insets compare the applied shear rate (solid line) with the slope obtained from the linear fit (points). Furthermore, additional points below the inner radius of $8 \mathrm{~mm}$ in the thicker slice case give a wrong impression of the actual gradient in the flow field. . . . . . . . . . . . . . . . . . .

5.8 Same as Fig. 5.7, however with a model fluid obeying the simple lever rule Eq. (3.14) with $\dot{\gamma}_{l}=2.5 \mathrm{~s}^{-1}$ and $\dot{\gamma}_{h}=35 \mathrm{~s}^{-1}$. Also, the profiles in (b) and (d) are fitted with a piece-wise linear function assuming two bands instead of simple linear function. The insets show the shear rate $\dot{\gamma}_{h}$ in the high shear rate band. Note that the $1 \mathrm{~d}$ profiles have been truncated at $8.6 \mathrm{~mm}$ to have an enlarged view on the high shear rate region. . . . . . . . . . . 
5.9 The transverse magnetization at the echo time obtained from the soft pulse simulation for the 'sinc3G10' pulse (a). Averaging over the ranges indicated by the dotted vertical grid lines removes the oscillations observed for $M_{x}$ and $M_{y}(\mathrm{~b})$. Please note that $z$ refers to the internal coordinate of the simulation program and not to the $z$-direction of the experimental setup shown in Fig. 5.4. $s$ refers to the thickness of the slice. . . . . . . . . . . . 85

5.10 Slice deformation at $\dot{\gamma}_{a}=10 \mathrm{~s}^{-1}$ for the shear banding model fluid $25 \mathrm{~ms}$, $100 \mathrm{~ms}$ and $200 \mathrm{~ms}$ after the PGSE slice selection. The times approximately correspond to the beginning, the middle and end of the $k$-space acquisition, respectively. . . . . . . . . . . . . . . . . . 88

5.11 PGSE-RARE sequence variant used in the 'velosim' program. In contrast to the sequence used for the experiment (Fig. 5.3) hard refocusing pulses are used and a 'Filter' element is added. . . . . . . . . . . . . . . . .

5.12 Typical outcome of the 2D PGSE-RARE simulation: Magnitude (a) and flow (b) map. Read and phase correspond to $x$ and $z$ respectively as defined in Fig. 5.4. The field of view is $20 \mathrm{~mm}$ in read and $30 \mathrm{~mm}$ in the phase direction. The height of the simulation cell is $25 \mathrm{~mm}$. The values $N_{r}, N_{\varphi}$ and $N_{z}$, which are defining the simulation grid, were set to 50, 50 and 100, respectively. . . . . . . . . . . . . . . . . . . 90

5.13 Flow during the RARE sequence can affect velocity measurements. (a) Spatially averaged velocity profiles for linear and centric $k$-space scheme at two values of $\dot{\gamma}_{a}$ obtained by NMR simulation using the model shear banding fluid. The piece-wise linear fits (solid lines) only include pixels that are fully situated in the gap range. Dashed lines correspond to the expected profile for each shear rate. (b) $\dot{\gamma}_{h}$ as a function of the applied shear rate, extracted by fitting of the spatially averaged velocity profiles for both $k$-space schemes. . . . . . . . . . . . . . . . . . . . . 91

5.14 Two methods for calibrating the gap location. Intensity calibration (a): The magnitude information of the NMR signal is used to determine the gap position. Intersections with the $50 \%$ threshold of the gap averaged signal intensity $I_{\text {gap }}$ are identified as the position of the wall-fluid interface. Marker calibration (b): Linear extrapolation of the marker fluid profile intersects the linear gap profile at the position of the inner wall. Outer wall position is given by the zero-intersect of the gap profile. The applied shear rate was $1 \mathrm{~s}^{-1}$. Dashed horizontal lines indicate the wall positions in both images. Please note that the data were extracted from the same experiment. 93 
5.15 (a) Spatially and temporally averaged velocity profiles for a shear banding fluid acquired with the PGSE-RARE sequence from Fig. 5.3 for various slice thicknesses. The solid lines correspond to piece-wise linear fits assuming two different slopes. (b) The slope of the high shear (velocity gradient) band as a function of the applied shear rate for various slice thicknesses. Errorbars are given by the uncertainty from the fitting routine. . . . . . . . . . . . 98

5.16 Same as Fig. 5.15, but for different $k$-space schemes. . . . . . . . . . . . . . 98

5.17 Averaged gap velocity profiles for different $\dot{\gamma}_{a}$ from intensity (a) and marker (b) calibration. The solid lines are piece-wise linear fits assuming two velocity gradients. Note that the inner wall position has been chosen as the origin for both gaps. . . . . . . . . . . . . . . . . . . . . . . 999

5.18 (a) Single 2D NMR flow field from a series of 50 individual velocity maps for Couette cell A (without spurious artefacts). Teflon tape wrapped around the inner cylinder at $z \approx-2 \mathrm{~mm}$ creating a ring of about $5 \mathrm{~mm}$ height and $0.5 \mathrm{~mm}$ thickness served as a flow feature in the phase direction. Velocities between the two gaps are from a marker fluid undergoing rigid body rotation. (b) Flow profiles at different positions in the phase direction. . . . 100

5.19 Signal attenuation due to free diffusion according to the Stejskal-Tanner relation (Eq. (2.28)) for the water acting as solvent in a WLM solution and a high viscosity PDMS solution in the case of the high resolution PGSERARE sequence. . . . . . . . . . . . . . . . . . . . 102

5.20 (a) 1D HR velocity profiles of water for different applied shear rates. Each profile is an average over 450 single velocity profiles, that were acquired consecutively with a repetition time of $2 \mathrm{~s}$. (b) Same as (a) but for the wormlike micellar solution instead of water. Also, the average is taken over 60 profiles acquired over $120 \mathrm{~s}$. Because of the high resolution in the read direction, the banded flow can already be described quantitatively at an applied shear rate of $4 \mathrm{~s}^{-1}$ slightly after the shear banding transition. Please note, that the velocity in certain pixels was set to zero, as a velocity determination was not possible at these positions due to external (rf) signal perturbations. . . . . . . . . . . . . . . . . . . . . . . 104 
5.21 2D high resolution velocity maps for high viscosity PDMS at applied shear rates of $6 \mathrm{~s}^{-1}$ (a), $12 \mathrm{~s}^{-1}$ (b) and $20 \mathrm{~s}^{-1}$ (c) as recorded with the centric $k$ space scheme. The phase positions of the $1 \mathrm{D}$ velocity profiles are the same for all plots. The dashed horizontal lines mark the expected velocity of the inner wall and the Teflon band with the latter being higher. The velocity of pixels with low image intensity were set to zero. . . . . . . . . . . 106

5.22 Same as Fig. 5.21, but for the linear scheme. . . . . . . . . . . . . . . 107

5.23 Same parameters as in Fig. 5.21a, but recorded using an off-balanced read gradient, as described in Ref. Shiko et al. (2012). Due to the splitting of the coherence pathways the number of pixels in the read direction is reduced by a factor of two down to $32 \ldots \ldots$. . . . . . . . . . . . . . . 108

6.1 Bulk rheology of the wormlike micellar solution under study. A steel coneand-plate device with $60 \mathrm{~mm}$ diameter and $2^{\circ}$ angle has been used to record the flow curve. Stress values were taken in a single sweep (low to high) and are an average over the last $30 \mathrm{~s}$ of each $60 \mathrm{~s}$ step. . . . . . . . . . . . . 118

6.2 (a) General setup of the USV apparatus. (b) Top view on the gap of the USV Couette cell together with the acoustic beam path (blue line). The incident angle $\phi$ ensures sensitivity for tangential velocity, as only displacements along the beam path can be measured. Furthermore, $\phi$ defines a slice thickness similar to the NMR experiments. In the setup used herein, this slice thickness is about $300 \mu \mathrm{m}$. Adapted from Gallot et al. (2013). . . . . . 121

6.3 Spatially and temporally averaged velocity profiles for NMR HR (a,c) and NMR LR $(b, d)$ at various applied shear rates. The averages are taken over $800 \mathrm{~s}$ after the transient startup behaviour with a repetition time of $2 \mathrm{~s}$ for each single flow map. Symbols represent the velocity averages of each voxel and the solid lines are piece-wise fits assuming two bands. Errorbars showing the standard deviation for each single data point have been omitted in the case of NMR HR due to their large extent caused by the low SNR of the method . . . . . . . . . . . . . . . . . . 125

6.4 Same as Fig. 6.3, but for applied shear rates in the range of $14 \mathrm{~s}^{-1}$ to $24 \mathrm{~s}^{-1} .126$ 
6.5 Comparison of NMR LR and HR data. The parameters $\dot{\gamma}_{l}$ (a), $\dot{\gamma}_{h}$ (b), $\alpha_{h}$ (c), $v_{s} / V(\mathrm{~d})$ as obtained from the piece linear fits of the averaged velocity profiles are plotted as a function of the imposed shear rate $\dot{\gamma}_{a}$ The low resolution data does not capture the increase of $\alpha_{h}$ for $\dot{\gamma}_{a}<15 \mathrm{~s}^{-1}$. For $\dot{\gamma}_{a}>15 s^{-1}$ there is relatively good agreement between HR and LR. The errorbars for $\dot{\gamma}_{l}, \dot{\gamma}_{h}$ and $\alpha_{h}$ show the errors from the fit. The errorbars for the slip are given by deviations for the fluid velocity at the wall if one assumes a error of half a pixel width for the position of the inner wall. Please note, that errorbars are sometimes smaller than the size of the symbols. . . . . . 128

6.6 Spatially and temporally averaged steady state profiles for USV measurements with smooth $(\mathrm{a}, \mathrm{c})$ and rough $(\mathrm{b}, \mathrm{d})$ inner wall surfaces at various imposed shear rates. The solid lines represent piece-wise linear fits of the flow profile. The average is taken over a window of $100 \mathrm{~s}$ starting $2.5 \mathrm{~min}$ after the inception of shear. Errorbars indicate the standard deviations and are measure for the amount of fluctuations present at the corresponding position. For $\dot{\gamma}_{a}>15 \mathrm{~s}^{-1}$ in the smooth case and $\dot{\gamma}_{a}>12 \mathrm{~s}^{-1}$ in the rough wall case turbulent bursts started altering the flow pattern after a certain time $t_{b}$. Therefore only a short period some time after the startup and $t_{b}$ is used for the average. . . . . . . . . . . . . . . . . . . . . . . . 129

6.7 Comparison of NMR HR and USV measurements. The parameters $\dot{\gamma}_{l}$ (a), $\dot{\gamma}_{h}(\mathrm{~b}), \alpha_{h}(\mathrm{c}), v_{s} / V(\mathrm{~d})$ as obtained from the piece-linear fits of the averaged velocity profiles are plotted as function of the imposed shear rate $\dot{\gamma}_{a}$. The errorbars indicate the fitting errors of the respective parameters, except for (d) (no errorbars). In (c) the dashed lines represent linear fits for different experimental condition (same colour coding). For NMR HR, only points where $\dot{\gamma}_{a}<17 \mathrm{~s}^{-1}$ are considered for the fit. . . . . . . . . . . . . . 131

6.8 (a) and (b) are the same as Fig. 6.7b and Fig. 6.7c, respectively, but with the true shear rate $\dot{\gamma}_{\text {true }}$ on the abscissa. For NMR HR in (b), only points where $\dot{\gamma}_{\text {true }}<10 \mathrm{~s}^{-1}$ are considered for the fit. . . . . . . . . . . . . 133

6.9 Vorticity structure of the flow velocity for a position $x / d$ close to the inner rotating wall at $\dot{\gamma}_{a}=15 \mathrm{~s}^{-1}$ for USV smooth (a) and NMR LR (b). $x / d$ is given in the graph titles. In (c) the temporal average over the given time interval is shown. Shear inception started at time origin. Errorbars indicate standard deviations, only every tenth errorbar is shown for USV for better visualization. Note, that the field of view in the vorticity direction was about $30 \mathrm{~mm}$ for USV and $25 \mathrm{~mm}$ for NMR. . . . . . . . . . . . . . . . 134 
6.10 The wavelength of vorticity undulations for USV smooth and USV rough was determined by Fourier transforming the autocorrelated velocity data for various shear rates. The first row shows the time-averaged axial velocity profile at a position close to the inner wall $x / d=0.08$ (same as in Fig. 6.9a). In the second row the corresponding autocorrelation function is depicted (arbitrary unit for ordinate). The third row shows the wavelength spectrum of the autocorrelation data which are truncated at $12 \mathrm{~mm}$ (arbitrary unit for ordinate). The undulation wavelength as shown in Fig. 6.11 is determined by picking the maximum of the spectral distribution. . . . . . . . . . . . 136

6.11 Wavelengths extracted from Fourier spectrum analysis of the undulation pattern for time-averaged vorticity profile for a pixel close to the inner wall (filled symbols). See Fig. 6.10 for more details on the analysis procedure. As expected, the undulation wavelength shows an approximate linear dependence on the applied shear rates. Hollow symbols indicate the corresponding expected wavelengths obtained from a master curve in Fardin et al. $(2012 b) \ldots \ldots \ldots 137$

6.12 Same as Fig. 6.10 for the $\dot{\gamma}_{a}=15 \mathrm{~s}^{-1}$ smooth case, but using the proportion of the high shear rate band $\alpha_{h}(z)$ instead of the velocity for a pixel close to the inner wall for the wavelength extraction. . . . . . . . . . . 137

6.13 Temporal evolution of the flow field over a short time period as recorded with USV TH at an imposed shear rate of $18 \mathrm{~s}^{-1}$ about 5 min after the startup. At position (i) the occurrence of a turbulent burst is captured. The flow of a certain fluid portion in the high shear rate band becomes unstable and massively alters the dynamics in its vicinity over the given time period. In general the flow is inhomogeneous in the vorticity and temporal directions even without bursts as exemplified by the profiles recorded at position (ii) and (iii) . . . . . . . . . . . . . . . . . . 138 
6.14 (a) Instability criterion $\Sigma$ (Eq. (6.2)) calculated from the fit values of the averaged velocity profiles (see Fig. 6.7) for the USV and NMR HR experiments. $\Sigma_{0}$ corresponds to the $\Sigma$ value for USV rough at $\dot{\gamma}_{a}=15 \mathrm{~s}^{-1}$, the lowest applied shear rate where bursts are detected. The blue hollow circles indicate where bursts are detected. The stress relaxation time $\lambda$ is assumed to be equal in all three cases. (b)\&(c): Comparison of averaged flow profiles for shear rate of $18 s^{-1}$ (b) and $20 s^{-1}$ (c) for USV rough and NMR HR. Errorbars for USV indicate standard deviations. For each imposed shear rate the two USV rough profiles correspond to two different averaging windows, one without and one with the bursts. The profiles have been normalized by the rotor velocities $V$ allowing for better comparison of USV and NMR results. . . . . . . . . . . . . . . . . . . . . . . . 140

6.15 Distributions for $\dot{\gamma}_{l}$ (a), $\dot{\gamma}_{h}$ (b), $\alpha_{h}$ (c) and the normalized slip (d) computed from the spatially and temporally resolved velocity profile fits for NMR LR at various applied shear rates. Broad distributions are obtained showing the fluctuative nature of the flow. In (e)-(h) the means of the distributions ("dist") are compared to the fits of the averaged velocity profiles ("fit"; from Fig. 6.5) for the whole range of imposed shear rates. The errorbars indicate the standard deviation of the distribution $((\mathrm{e})-(\mathrm{h}))$ and the fitting error (only (e)-(g)), respectively. The good agreement shows that the averaged velocity profiles represent the mean of a potentially broad distribution . . . 143

6.16 Same as Fig. 6.15, but for the USV smooth experiments. The observations are similar to those obtained of the NMR LR case. . . . . . . . . . . . . . . 144 



\section{List of Tables}

2.1 Commonly defined relation between the phase $\phi$ and the rotation axis of the $r f$ pulse in the rotating frame. . . . . . . . . . . . 9

3.1 Two common surfactant molecules used for creating micellar solutions. . . . 48

6.1 Comparison of spatial and temporal resolution for the techniques and methods applied. The temporal resolution refers to the repetition time $T_{\mathrm{R}}$ for successive flow maps. 'Approx. gap point' stands for the approximate number of data points over the Couette gap and thus is an indicator for the effective resolution of the method. . . . . . . . . . . . . . . 122 
